\title{
Continuous Hydrothermal Synthesis of Inorganic Nanoparticles; Applications and Future Directions
}

\section{Jawwad A. Darr ${ }^{\dagger \star}$, Jingyi Zhang, ${ }^{\ddagger}$ Neel M. Makwana ${ }^{\dagger}$ and Xiaole Weng ${ }^{\ddagger}$}

'Department of Chemistry, University College London, Christopher Ingold Laboratories, 20 Gordon Street, London WC1 1 oAJ.

${ }^{\ddagger}$ Department of Environmental \& Resource Sciences, Zhejiang University, 866 Yuhangtang Road, Hangzhou, Zhejiang 310058 , P. R. China

*corresponding author j.a.darr@ucl.ac.uk

\section{ABSTRACT}

Nanomaterials are at the leading edge of the emerging field of Nanotechnology. Their unique and tunable size-dependent properties (in the range 1 to $100 \mathrm{~nm}$ ), make these materials indispensable in many modern technological applications. In this review, we summarize the state-of-art in the manufacture and applications of inorganic nanoparticles made using continuous hydrothermal flow synthesis (CHFS) processes. Firstly, we introduce ideal requirements of any flow process for nanoceramics production, outline different approaches to CHFS and introduce the pertinent properties of supercritical water and issues around mixing in flow to generate nanoparticles. The review then gives comprehensive coverage of the current application space for CHFS-made nanomaterials including optical, healthcare, electronics (including sensors, information and communication technologies), catalysis, devices (including energy harvesting/conversion/fuels) and energy storage applications. Thereafter, topics of precur- sor chemistry and products, as well as materials or structures are discussed (surface functionalized hybrids, nanocomposites, nanograined coatings and monoliths, metal-organic frameworks). Latterly, the review focusses on some of the key apparatus innovations in the field, such as in situ flow / rapid heating systems (to investigate kinetics and mechanisms), approaches to high throughput flow syntheses (for nanomaterials discovery), as well as recent developments in scale-up of hydrothermal flow processes. Finally, the review covers environmental considerations, future directions and capabilities, along with the conclusions and outlook. 
5.3. Bioceramics (Calcium Phosphates)

0021

5.4. $\mathrm{ZrO}_{2}$ Biomedical Applications

0024

5.5. Nanophosphors/O Dots (Bio)

0025

1 Introduction

0002

1.1. An Ideal Process for Nanoceramics

0003

1.2 Continuous Hydrothermal Flow Synthesis

0004

Communication Technology.

0026

6.1. Metals and Transparent Conducting Oxides 0026

2 Properties and Reactions of sc-Water

0005

6.2.Semiconductors (Electronics, Gas Sensors)

0029

2. 1 Properties of sc-Water

0005

2.2 Chemistry and Process Variables

0006

6.3. Dielectrics, Piezoelectrics, Ferroelectrics

and Multiferroics

0031

6.4. Magnetic Particles (Non Bio)

0035

3 Overview of Continuous Hydrothermal

Reactors

000

7 Catalysis Applications

0036

3.1. Early Approaches/General Observations

0007

0008

3.2. Mixing and Modelling

4 Optical Applications (Non-Bio Uses)

0010

4.1. Nanophosphors (Non-Bio Uses)

0010

7.1. Photocatalysts

0036

7.2. Heterogeneous Catalysts

0042

7.2.1. Non-Precious Metal Catalysts / Supports

0043

7.2.2. Precious Metal Containing Particles for

Catalysts

0049

4.2. UV Attenuating Nanoparticles

0013

4.3. Refractive Index Modifiers

0015

4.4. Non-linear optics

4.5. Nano Pigments and Thermochromics

5 Nanoceramics for Healthcare

8 Devices (Energy Harvesting/Conversion/Fuels)

0052

5.1. Magnetic Particles for Healthcare

5.2. Antimicrobials
0015

0017

0015

8.1. Solid Oxide Fuel Cell Materials

0052

8.2. $\mathrm{O}_{2}$ Separation / Membranes

0054

8.3. Thermoelectrics and Energy Scavenging 0055

0018

0020
8.4. Dye Sensitized or Other Solar Cells

0056

9 Energy Storage Applications 
9.1. Nanomaterials for Energy Storage

9.2. Lithium Ion Battery Cathodes

9.3. Lithium Ion Battery Anodes

9.4. Beyond Li lon Batteries

9.5. New Supercapacitor Nanomaterials

10 Misc. Nanomaterials Chemistry and Process

Developments

10.1. Misc Precursors, Co-solvents, Additives for Novel Chemistry or Phase Targeting

10.2. Surface Functionalized Hybrid NPs and

Nano-composites

10.3. Nanograined Coatings and Monoliths

10.4. Metal-Organic Frameworks (MOFs)

10.5. In Situ Flow / Rapid Heating Studies
11 Future Directions and Capabilities

0079
0056

0057

0062

0066

0067
10.6. High Throughput Flow Syntheses for

Materials Discovery

0075

10.7. Scale-up of Hydrothermal Flow

0077

10.8. Environmental Considerations

$0068 \quad 12$ Conclusions and Outlook

0081

0068 Author Information

Corresponding author

0082

0069 Abbreviations

0082

0072 Biographies

0084

0073 Acknowledgements

0085

$0074 \quad$ References

0085 


\section{INTRODUCTION}

Nanomaterials are at the leading edge of the emerging field of Nanotechnology. Their unique and tuneable sizedependent properties ${ }^{1}$ (in the range 1-100 nm), make these materials superior and indispensable in many modern technological applications. The significant reduction in size of bulk materials, often improves their properties due to quantum confinement effects, giving rise to tuneable electronic or other properties. ${ }^{2}$ Nanomaterials can also have greatly increased surface area to volume ratios, which can determine the rate of surface area dependant (or defect site controlled) reaction rates.

Nanotechnology is being developed in several forms; materials (e.g. powders/dispersions), coatings, (nanograined) monoliths, polymer-ceramic composites, devices and systems. ${ }^{3}$ Nanotechnology is already playing an increasing impact on our everyday lives, particularly in an age where energy and chemical or other resource efficiency has become vital in sustaining the world's ever increasing population. ${ }^{3}$ At the present time, advanced nanomaterials are beginning to enter the commercial marketplace as consumers demand for ever increasing performance and miniaturisation from products; from self-cleaning coatings 4-5 $^{-5}$ to more efficient catalysis, ${ }^{6-9}$ particles for oil recovery, ${ }^{10}$ photocatalysis, ${ }^{4,}{ }^{11-16}$ healthcare products including sunscreens, ${ }^{17-18}$ battery materials, ${ }^{19-20}$ electronic storage media and solid state sensors. ${ }^{21-23}$

With increased recent worldwide interest in both green and nano-technologies, ${ }^{24}$ more sustainable manufacturing routes to crystalline nanometric inorganic materials are of interest. ${ }^{25-27}$ The vast majority of published literature on the syntheses of nanoparticles (NPs), relate to materials prepared under static or batch processes ${ }^{25-27}$ or indeed multi-step processes. More recently, an increasing number of publications have demonstrated the potential of rapid continuous methods for controlled manufacture of inorganic nanomaterials. ${ }^{28-32}$ Amongst these reactions, the greenest and most promising can be considered those which are primarily water based, i.e. continuous hydrothermal synthesis processes. Many acronyms are used in the literature for such processes and the authors prefer the use of CHFS (continuous hydrothermal flow synthesis) for such processes, i.e. those that use predominately water not organic solvents (this will be used periodically hereafter). Its first important to how continuous processes for NP production tend to operate; in many continuous hydrothermal processes, a pre-heated water supply under pressure is mixed with an ambient temperature aqueous (aq) metal salt solution under flow conditions in an engineered mixer, whereupon a NP metal oxide (typically) is the product, depending on the process conditions and reagents used. 
The metal salt solution is added to the process at close to ambient temperature, otherwise it can undergo significant pre-heating before it meets a superheated water feed, which may result in undesirable accumulation in pipes or give poorly defined growth conditions. In typical reactions of superheated water and metal salts, an engineered mixer is used (sometimes denoted as a "reactor"), and a high temperature, typically in the range 200 to $400{ }^{\circ} \mathrm{C}$ is obtained (after mixing) with a short residence time typically in the range a few seconds to minutes (rather than hours) for nanoparticles for form.

Such hydrothermal flow processes at high temperatures, are in stark contrast to geological high pressure processes, which can happen over millions of years. This means that in continuous hydrothermal processes, metastable or kinetic compositions can be formed and that the NPs formed, can display unique structures or combinations of particle properties that cannot be readily achieved using more conventional batch hydrothermal or other synthesis methods. This might include enhanced solid solution solubility over that reported using more conventional synthesis methods. 33-34 Many examples of different engineered mixers (reactors) are known and different research groups around the world that are very active in this area. A detailed review of mixers is not given herein, as it would command a review in its own right; therefore, only limited discussion of this topic will be herein.

In the last 25 years or so, there has been a steady increase in the number of process designs and different applications for NPs made via continuous hydrothermal processes. With such hydrothermal flow processes, the number of process variables that can be independently varied are considerable $(T, P$, flow rate, composition, turbulence of mixing, etc.), thus, CHFS offers an almost unlimited toolkit for materials scientists to be able to design or tailor NPs for specific requirements (see Table 1). For example, a single continuous hydrothermal process could be used to carry out more than one function; e.g. to

1) Control nucleation to make narrow size particles of a specific crystal structure (in a first mixer),

2) Add a surfactant coat that can then be applied in flow (via a second mixer) so nanoparticles can be dispersible in organic solvents or water (depending on the surfactant).

More information about different options for coated NPs will be contained later in this review (section 10.2).

Given the potential for CHFS reactors to scalably manufacture nanomaterials, an exciting era of engineered NPs for commercial applications is upon us; not only for ceramics or metals, but also in advanced (hybrid) composites 
and metal organic frameworks and other materials with particle properties (composition, size, shape, aspect ratio, porosity, phase solubility, etc.). These combinations of particle attributes cannot be readily achieved using other direct synthesis processes. Alongside, these new opportunities to develop nanomaterials commercially, will arise questions about their safety during manufacture, use and also their fate at the end of their life (e.g. landfill). 35 In order to address this, safeguards for manufacture, use, handling, processing, and processes / regulations for end of life will be required to ensure that the environmental impacts of such new nanomaterials are considered.

This comprehensive review seeks to bring together many of the recent peer reviewed papers in the field whilst demonstrating some key applications and give a balanced argument about the pros and cons of applying continuous hydrothermal synthesis methods for nanoceramics syntheses. This review will cover some recent exciting developments as well offer signposts where the technology has industrial. This review will provide a good starting point for non-specialists and industrialists wishing to understand and explore the potential of CHFS technologies towards tailored inorganic materials applications. These applications include using NPs directly (e.g. dispersed ${ }^{36}$ in a formulation) or towards building nano-grained devices, composites, coatings ${ }^{37}$ or for bulk materials as a "bottom up" solid state synthesis approach. ${ }^{38}$

1.1 An Ideal Process for Inorganic Nanoparticle Synthesis

Despite the availability of a number of methods which are potentially capable of producing NPs at various stages of academic or commercial development 39 (e.g. plasma or flame based synthese ${ }^{40-43}$ ) the quality and consistency of NPs can differ from process to process. Any commercially viable and safe process for the synthesis of high quality nanomaterials should preferably display the following characteristics many of which are in keeping with the principles of green chemistry. ${ }^{44}$ The process should;

(i) Allow controllable manufacture of homogenous nanomaterials ( $<100 \mathrm{~nm}$ diameter particle) with desired composition and characteristics,

(ii) Be energy and resource efficient (i.e. avoids the use of wasteful organic solvents or large amounts of energy and ideally use green solvents such as water)

(iii) Be fast and flexible and use relatively few steps (preferably a one-step or rapid process),

(iv) It should possess consistent quality with ideally no batch-to-batch variations (e.g. as in a continuous process) 
(v) If possible, it should allow constant "in process" or "in line" monitoring for quality control measures,

vi) Allow flexibility to affect or control particle attributes by changing process conditions, e.g. pressure, flow rate etc. (see table 1)

vii) Give access to unique nanomaterials that are difficult to make directly using other methods (unique phase compositions, unique combination of particle properties)

(viii) Should minimize hazards to workers or end users e.g. being of minimal internal volume or readily shutdown in an emergency, or produce NPs that cannot be readily inhaled by operator.

The inability of many processes to manufacture high quality nanomaterials in a consistent and safe way is in part due to inherent limitations of processes or lack of knowledge on particle-parameter behaviors. For example, processes which involve very high temperatures such as flame, combustion or plasma processes, result in phase separation, or extensive agglomeration with low surface areas. ${ }^{45}$ Some of these methods also produce highly charged NPs in the gas phase which could potentially be hazardous to workers as they are airborne and present a potential inhalation hazard. $4^{6}$

More conventional NP synthesis methods such as sol-gel and co-precipitation techniques, are slow, require multiple steps and need very precise control of several reaction parameters $(\mathrm{pH}$, temperature, etc.), otherwise yielding inconsistent products. ${ }^{47-48}$ Emulsion syntheses of NPs can be very wasteful, 49 often requiring large amounts of organic solvents, whilst ball milling ${ }^{50}$ (top down) is energy intensive and slow and makes materials in a dry powder form, which could pose an inhalation hazard. Increasing the control, speed and homogeneity of NP manufacturing methods, will significantly enhance product quality and consistency of de novo engineered nanomaterials.

As explained above, many current synthesis processes for nanomaterials, fail to meet all the requirements for safe synthesis of high quality nanomaterials. Indeed, concerns over the safe working with nanomaterials were highlighted in a joint Royal Society of Chemistry / Royal Academy of Engineering report on Nanotechnology, and need to be addressed for any manufacturing process to reach long term acceptance. ${ }^{11}$ Since this report, the sheer volume of databases and guides as well as regulatory accumulation of data on nanomaterials has increased at pace. Noteworthy documents at this time, which are recommended include a number of assessments to provide a more solid foundation for discussion of the regulation of nanomaterials in Europe. The authors particularly recommend the document "Dialogforum Nano of BASF 2014/2015 on fostering transparency and responsible innovation with nano- 
materials", ${ }^{35}$ which is an assessment of various European tools and registers in terms of how they address the transparency needs of three target groups: public, authorities, consumers and workplaces. ${ }^{35}$ Within this document are many useful links such as Information about nanomaterials and their safety assessments, as well as "general Information on nanomaterials", "how are nanomaterials released?", "How can nanomaterials enter the body or the environment?", "How do nanomaterials behave once inside the body or the environment?". Many of these questions need to be better understood as we begin to develop processes, which are capable of reproducible larger scale production of NPs.

In the manufacture of high quality homometallic or heterometallic oxide nanomaterials, hydrothermal processes offer significant benefits because they can operate at relatively low synthesis temperatures (compared to flame and plasma processes) and follow cleaner processing routes, not involving organic solvents. .2-56 $^{25}$

In a typical CHFS process, water is heated above its boiling point under pressure to cause precipitation or crystallisation of inorganic materials, akin to processes in which minerals are formed under the earth's crust over long periods or indeed in hydrothermal vents, which are deep under the sea. These natural vents occur in geologically active regions of the ocean floor where seawater penetrates fissures in the rock and is then heated by magma. Over time, the water dissolves minerals from the rock and as the water rises, some of the minerals precipitate out and harden on the rim of the vent. Eventually, a tall, chimney-like structure is formed; these are nicknamed "black smokers". The temperature of the water coming out of these deep-sea hydrothermal vents can exceed $360^{\circ} \mathrm{C}$, therefore, there are obvious parallels to the conditions in a laboratory based continuous hydrothermal reactor.

Superheated or supercritical water (sc-water) is of interest in a range of applications, e.g. oxidation of fine chemicals or sludge treatment. ${ }^{57}$ Using water in processes is clearly also more sustainable than using organic solvents (such as in sol-gel 47 or emulsion ${ }^{49}$ methods). However, to date the vast majority of hydrothermal nanomaterials synthesis processes are batch-wise operations, which have inherent drawbacks of being slow (one a day), difficult to scale-up (due to large volumes under high pressure), and difficult to control nucleation and growth precisely, leading to batch to batch variations, i.e. inconsistent products. $53-54$

There are a number of particularly excellent research articles and reviews and articles on supercritical (sc) fluids and inorganic materials $\mathrm{s}^{28,58-62}$ as well as reviews and articles on the production of micro- and nanoparticles using supercritical water, ${ }^{61}$ sol-gel chemistry in Supercritical Fluids (including carbon dioxide mixtures, ${ }^{63}$ organic solvents and water), ${ }^{60}$ nanomaterials syntheses in supercritical flow from coordination complexes, ${ }^{59}$ and nanomaterials from 
batch hydrothermal and hydrothermal flow $5^{8}$ methods. The authors recommend a review by Byrappa and Adschiri, which discusses liquid phase syntheses and compares several different synthetic routes. ${ }^{25}$ Another review by Adschiri on "Supercritical Hydrothermal Synthesis of Organic-Inorganic Hybrid NPs" is also recommended to better understand the potential routes to make surface coated NPs (many of which are made in batch reactions, therefore, we will focus later mainly on flow processes in this regard). ${ }^{64}$ The review by distinguished Professor Yoshimura (a world authority in hydrothermal science), is also highly recommended. ${ }^{26}$ The authors refer readers to the series of conferences hosted around the world under the International Solvothermal and Hydrothermal Association (ISHA), which is mainly concerned with exotic reaction chemistry associated with organic solvents (solvothermal) and superheated water, respectively. These meetings can generally be located by a cursory search on the World Wide Web.

\subsection{Continuous Hydrothermal Synthesis}

In contrast to batch hydrothermal processes, continuous hydrothermal processes have been used for over 25 years or so for the efficient manufacture of NPs. Basic continuous hydrothermal processes can vary and designs for processes are emerging all the time. A typical process uses a high pressure pump to deliver room temperature water through an in-line heater in order to raise its temperature to typically $>400{ }^{\circ} \mathrm{C}$ or more (well above its critical point). In a continuous reactor, a flow of superheated water is then brought to meet a flow of metal salt solution (aq) (which can be premixed in flow with a pH modifier or other reagent). Thereafter, the sc-water and metal feeds (the latter of which may have been premixed with base) meet in an engineered mixer, resulting in hydrolysis and dehydration or other reactions to form a metal oxide (or hydroxide, or oxyhydroxide, sulphide, phosphate, etc.). After initial nucleation, some growth can occur before the NPs are cooled in flow and then recovered as an aqueous slurry at atmospheric pressure (after exiting the process via a back-pressure regulator valve). A number of hydrothermal flow systems designs are known to make metals and metal oxides, for example with supercritical alcohols, ${ }^{65-70}$ however, we consider them largely out of scope, as we are mainly concerned with sc-water and its mixtures.

Figure 1 
Since the early research into continuous hydrothermal systems, ${ }^{71-74}$ the number of researchers, engineering designs and materials applications has increased. $.^{30,} 32,75-90$ The advantages of using continuous hydrothermal over batch hydrothermal methods are apparent and numerous and will be discussed later. At this time, CHFS technology is rapidly expanding into a number of new application areas and approaches to materials development; from nano-biomaterials and printed electronics to solid oxide fuel cell cathode materials ${ }^{38,}{ }^{11}$ and photocatalysts for hydrogen production / pollution remediation. ${ }^{92}$ This review paper seeks to take an overview of some of the differing synthetic approaches for CHFS and to look forward to how NPs made this way might find industrial applications.

It is envisaged that inorganic nanomaterials and their composites or derivatives, will become ever more important due to socioeconomic factors, e.g. pollution prevention / remediation, microelectronics and devices, for enhanced security, in biological / healthcare applications, more efficient devices for energy storage and energy reduction / transfer. This review will also cover some of the chemistry aspects related to continuous flow reactors including novel approaches to understanding growth processes, new approaches to nanomaterials discovery as well as process scale-up. ${ }^{32,93}$ Before we discuss applications, it's worth considering the key properties of superheated water.

\section{PROPERTIES AND REACTIONS OF SC-WATER}

In order to understand why continuous hydrothermal processes can produce small NP ceramics in a matter of a few seconds, we must first understand the properties of supercritical water, which is one of the feeds / reagents for a continuous hydrothermal flow process.

\subsection{Properties of sc-Water}

Supercritical (sc) fluids are defined as the state of matter which exists above a substance's critical temperature and pressure. ${ }^{28}$ This state is neither a liquid nor a gas, but it can simultaneously display the properties of both. In relation to the chemistry of inorganic species, a range of Supercritical Fluids have been explored, e.g. sc-alchohols, etc. ${ }^{66-67,94-95}$ In each case, the fluids have been used as clean solvents (e.g. for impregnating drugs into polymers, ${ }^{96-}$

${ }^{100}$ or as an oil phase for generating templated monoliths), ${ }^{101-103}$ reagents or even as "inert" anti-solvents and plasticisers, ${ }^{104}$ to name a few different roles. ${ }^{28}$ A comprehensive review by one of the authors is highly recommended as a general introduction to the Supercritical Fluids and inorganic materials and compounds field. ${ }^{28} \mathrm{~A}$ paper by Adschiri 
et al. also describes the properties and some specific features of supercritical water. ${ }^{105}$ Publications on supercritical water are numerous, since water is abundant and environmentally friendly, making it the ideal green solvent / reagent for chemical processes. For water, the critical temperature and pressure are ca. $647 \mathrm{~K}\left(374^{\circ} \mathrm{C}_{i} 705^{\circ} \mathrm{F}\right)$ and 22.1 MPa (3200 psi or $218 \mathrm{~atm}$ ), respectively. ${ }^{106}$ Approaching these critical values, the properties of water begin to change radically (see figure $2 \mathrm{a}, 2 \mathrm{~b}$ and $2 \mathrm{c}$ ) in a manner, which makes it a potentially useful medium for conducting rapid chemical reactions.

Near the critical point, the properties of water are very different to that when it is under ambient conditions. The density of water decreases with increasing temperature from $1000 \mathrm{~kg} \cdot \mathrm{m}^{-3}$ under ambient conditions to $712 \mathrm{~kg} \cdot \mathrm{m}^{-3}$ at a temperature of $573 \mathrm{~K}$ on its "saturation curve" (the curve whereby the pressure is just high enough to keep the water as a liquid). Above $573 \mathrm{~K}$, the density drops rapidly until it is just $322 \mathrm{~kg} \cdot \mathrm{m}^{-3}$ at the critical point ${ }^{107}$ (see Figure $2 a$, 2b). Similarly, the dielectric constant of water decreases from 80 to 33 (the same as methanol under ambient conditions) at $483 \mathrm{~K}$, to ca. 5 (similar to ethyl acetate) at the critical point, and below 2 (akin to that of hexane; figure 2b) at $773 \mathrm{~K} .{ }^{107}$ This is because of the breakdown of extensive hydrogen bonding under these extreme conditions. The implication of the variations in these two properties is that polar inorganic salts are far less soluble in water under high temperature and pressure than at room temperature and pressure.

Figure 2

The key to production of NPs in continuous hydrothermal processes is the maximization of supersaturation, as this increases the rate of nucleation of the particles, leading to smaller particles. ${ }^{108}$ Since supersaturation is the ratio of the concentration of a species in a solution to the saturation concentration, it follows that the lower the saturation concentration, the higher the supersaturation will be. ${ }^{109}$ This means that a rapid change in conditions for metal salts from ambient to near- or supercritical conditions, should provide a strong hydrolyzing environment for the precipitation of inorganic NPs.

There are a number of other important changes in the behavior of ions in near-critical or sc- $\mathrm{H}_{2} \mathrm{O}$, which will now be discussed. Under ambient conditions, a cation in solution will typically be mainly surrounded by water molecules, with little presence of the anion in the first solvation shell. For example, $\mathrm{Zn}^{2+}$ in $\mathrm{ZnBr}_{2}$ has, on average, five water molecules and one $\mathrm{Br}^{-}$anion in its first solvation shell at ambient conditions. ${ }^{110}$ In contrast, $\mathrm{Ni}^{2+}$ in $\mathrm{NiBr}_{2}$ under ambi- 
ent conditions has six water molecules and no anions in the first solvation shell. ${ }^{111}$ Under supercritical conditions, these cations change to tetrahedral geometry, with the first solvation shell now containing almost four anions for $\mathrm{Ni}^{2+}$ and two for $\mathrm{Zn}^{2+} .{ }^{110-112}$ The situation is similar for $\mathrm{Ag}^{+}$in solutions of $\mathrm{AgNO}_{3} \cdot{ }^{113}$ In addition, the $\mathrm{M}-\mathrm{O}$ (water) bond length is shorter in the cases described above, while the $\mathrm{H}-\mathrm{O}$ bond of the water molecules is longer, suggesting that reactions between the metal and the water may be favored. As for anions, their solvation shells are largely disrupted and their solvation energies become near-zero. This means that, for example, acids become much less acidic in hydrothermal conditions; for example, the $\mathrm{pK}_{\mathrm{a}}$ of $\mathrm{HCl}$ is half of its ambient value at the critical point. ${ }^{114}$ In the case of both cations and anions, the major changes in their solution structure have taken place by the critical point.

In CHFS reactors, when metal salts under ambient conditions are suddenly mixed with sc-water, the conditions favorable for NP formation; rapid hydrolysis of the metal salts (taking metal nitrate salts as an example) and dehydration to hydroxides or oxides (or phosphates, etc.), can occur, often with narrow particle size distributions. The rapid hydrolysis of metal salts is supported by considering the values of $\mathrm{K}_{\mathrm{w}}$ (equilibrium constant for water) under extreme conditions. $K_{w}$ can be defined by the following equation;

$$
\mathrm{Kw}=\left[\mathrm{H}^{+}\right]\left[\mathrm{OH}^{-}\right]
$$

Where each term in square brackets at room temperature is ca. $1 \times 10^{-7} \mathrm{~mol} . \mathrm{dm}^{-3}$ (giving a value of $\mathrm{K}_{\mathrm{w}}=\mathrm{a}$ value $=1 \mathrm{x}$ $\left.10^{-14} \mathrm{~mol}^{2} . \mathrm{dm}^{-6}\right)$. Notably, the value of $\mathrm{K}_{\mathrm{w}}$ varies with temperature as we will see below. The $\mathrm{pK}$ is also defined by equation (2) below and so for a value of $\mathrm{K}_{\mathrm{w}}$ at room temperature of $1 \times 10^{-14} \mathrm{~mol}^{2}$. $\mathrm{dm}^{-6}$, this gives a pK walue of 14 at room temperature for example.

$$
\mathrm{pK}_{\mathrm{w}}=-\log _{10} \mathrm{Kw}
$$

Figure $2 \mathrm{c}$ shows that the $\mathrm{pK}_{\mathrm{w}}$ value versus temperature for various different pressures. It can be seen that water in the range at ca. $250^{\circ} \mathrm{C}$ up to near the critical temperature and at $69 \mathrm{MPa}$ (i.e. where the value of pK $\mathrm{w}_{\mathrm{w}}$ is ca. 11. In terms of the changes in the $\left[\mathrm{H}^{+}\right]$and $\left[\mathrm{OH}^{-}\right]$for water, this means that under the high temperature and pressure range, $\mathrm{K}_{\mathrm{w}}$ is approximately equal to $\mathrm{ca} .\left[3.2 \times 10^{-6}\right] \times\left[3.2 \times 10^{-6}\right]=1 \times 10^{-11} \mathrm{~mol}^{2} . \mathrm{dm}^{-6}$. Thus, we can surmise that the individual values of $\left[\mathrm{H}^{+}\right]$and $\left[\mathrm{OH}^{-}\right]$ions in the water go from ca. $1 \times 10^{-7}$ to $3.2 \times 10^{-6} \mathrm{~mol}^{-\mathrm{dm}^{-3}}$, which is a ca. 30 fold 
increase in the concentration. These changes provide a very hydrolysing environment, which favours the initial hydrolysis of the metal salt when it is mixed in flow with a supercritical water feed.

\subsection{Chemistry and Process Variables}

The reactions taking place in a continuous hydrothermal engineered mixer where sc-water and metal salts meet, can be summarized as a combination of rapid hydrolysis (often several steps) and dehydration, for which a typical and simplified set of equations are shown, for example in the reaction of a metal nitrate salt.

Hydrolysis:

$\mathrm{M}\left(\mathrm{NO}_{3}\right)_{\times(\mathrm{aq})}+\mathrm{xH} \mathrm{H}_{2} \mathrm{O} \rightarrow \mathrm{M}(\mathrm{OH})_{\times(\mathrm{s})}+x \mathrm{HNO}_{3}$

Dehydration: $\quad M(O H)_{x(s)} \rightarrow M(O)_{x / 2(s)}+x / 2 \mathrm{H}_{2} \mathrm{O} \quad$ (4)

However, this is an over simplification as the anions can often themselves decomposition smaller molecules and so the actual reactions that occur and number of steps or metal containing intermediates is often complex. For example, citrate or acetate precursors can often decompose in part to $\mathrm{H}_{2}$ or $\mathrm{CO}$ or other molecules, which may actually play a role in the reaction (such as acting as in situ reducing agents). Furthermore, indium salts may decompose to the oxide, via the hydroxide and then the oxyhydroxide, and depending on the exact conditions used, one of these intermediates may be retained in the collected products ${ }^{93}$ (i.e. the transformation via dehydration, may not always proceed all the way to the oxide).

A full discussions of growth models are not given here as they are already available elsewhere ${ }^{115}$ and because the focus of this review is on applications. Some salient points are below though for those who are new to the field. Key to the production of well-defined NPs is the mixing of sc-water and a metal salt solution (at ambient temperature) in an engineered mixer. When this happens, the level of supersaturation surpasses the critical nucleation threshold, nucleation occurs, which in turn reduces the degree of supersaturation. In a dynamic system such as a flow reactor, the precursor concentration will then become lower than the nucleation threshold. If there is no more material in solution, then the particles obtained, may primarily be those from the initial nuclei, which will result in a narrow size 
distribution and small particles. ${ }^{116}$ However, it is possible for NP growth to follow particle nucleation in a number of ways,

1) Precursor metal ions from solution can feed to grow the preformed nuclei, or

2) Ostwald ripening can occur (small nuclei that are able to dissolve and then redeposit onto more thermodynamically favorable larger nuclei, and

3) NPs can coalesce to form larger ones.

From the above scenarios, we can visualize a simplistic model which accounts for the varying size and shapes and size distributions often observed in continuous hydrothermal systems. ${ }^{115}$ Hydrothermal batch reactions, do not usually rapidly create this supersaturation due to slower heating times and they typically create micron-sized particles. ${ }^{25-27,} 117$ In cases where small particles are created in batch processes, organic surfactant molecules are often used to bind to the growing particles and thereby, retard their growth and agglomeration. ${ }^{25,} 64,118$

Table 1 suggests that there are relationships between process variables, impact and product outcomes for a typical continuous hydrothermal process (adapted from Lester et al.). ${ }^{119}$ The table clearly shows how one or more inputs can have a direct or indirect effect on the product's attributes. Just about every input variable in a continuous reactor can be independently altered, including process pressure, temperature of incoming water, concentration / composition of metals, relative flow rates, etc. This gives some control over the final products, which is a major strength of continuous hydrothermal processes.

The role of additives and the solubility of the metal salts, can both have an effect on crystallite size (see later section 10.1). For example, the authors have published the effects of adding peroxide in the CHFS synthesis of $\mathrm{ZnO}$ (from the metal nitrate salt); it appears that peroxide increases solubility of the $\mathrm{Zn}$ species in solution and hence, extends the growth phase on certain facets, which results in needles / rods or higher aspect ratio particles. ${ }^{120}$ Other additives are important in providing reducing or oxidizing environments for controlling phase behavior of the product, partially through affecting oxidation states of metals. Such additives can be introduced at various points in a flow process, e.g. a reducing agent can be added to the supercritical water feed prior to mixing with the metal salts. This has been used in a number of publications such as in transparent conducting oxides (see later in section 6.2). ${ }^{121}$ This approach can effectively enhance the potency of the chemical agent before its meets the metal salt in flow. 
Finally, it will be seen that more than just metal oxides can be made via continuous hydrothermal processes; indeed sulfides ${ }^{115,}{ }^{122}$ (see section on $\mathrm{Q}$ dots/phosphors), tellurides, ${ }^{123}$ silicates $^{124^{-126}}$ and phosphates ${ }^{76}$ (e.g. bioceram$\left.\operatorname{ics}^{76,127-130}\right)$ and metals ${ }^{131}$ (Cu for example) ${ }^{131}$ can also be made to name a few. These reactions require the addition of suitable reagents, as will be seen herein.

Table 1

\section{OVERVIEW OF CONTINUOUS HYDROTHERMAL REACTORS}

\subsection{Early Approaches / General Observations}

Historically, there were two approaches to early continuous hydrothermal processes, which were developed independently in USA and Japan, respectively. The simplest approach was developed at Pacific Northwest Laboratories, USA, in which a stream of metal ions in solution was pumped through a furnace (Figure $3 a) .{ }^{132}$ Narrow pipe diameters were used to allow the solution to rapidly reach the furnace temperature (high surface area to volume ratio), and particles were able to rapidly nucleate and grow. The growth of particles was rapidly terminated by a RESS (Rapid Expansion of Supercritical Solution) set-up at the end of the process, whereby the suspension passed out through an expansion nozzle into a condenser, enabling particles to be collected. ${ }^{133}$ This method was used to produce a number of materials, including simple oxides such as $\mathrm{Fe}_{2} \mathrm{O}_{3}, \mathrm{Fe}_{3} \mathrm{O}_{4}, \mathrm{CuO}, \mathrm{NiO}, \mathrm{ZrO}_{2}, \mathrm{TiO}_{2}$ and $\mathrm{ZnO}$, heterometallic oxides such as $\mathrm{NiFe}_{2} \mathrm{O}_{4}{ }^{133}$ and other materials such as iron oxyhydrides. ${ }^{134}$ This process design benefited from its simplicity in only having one feed, and no consideration of mixing dynamics required. However, this approach was limited by the necessity to rapidly quench the reactants, and it was inflexible.

A different (and now more widely used) approach was developed by Japanese researchers in the early 1990's (shown in Figure $3 b$ ). This system worked by mixing a stream of near-critical or supercritical water with a stream of inorganic salts (in solution at ambient temperature) in a continuous manner. ${ }^{72}$ The early continuous hydrothermal flow reactor apparatus as reported by researchers in Japan, typically consisted of two HPLC pumps, one of which pumped a metal salt solution (or mixture of metal salts), while the other delivered sc-water through an inline heater. In the early reactor designs, this preheater was simply coiled tubing in a heated furnace, but more recently, some research groups have used custom built electrical heaters, in which a coil is encased in or around a heated block. ${ }^{75-76,}$ $135-136$ 
Historically in continuous hydrothermal processes for NP synthesis, the two feeds were mixed (i.e. hot water and metal salts) in simple tee-piece arrangements (although more recently, other mixing designs have been investigated, as will be briefly mentioned later). In some cases, the processes also included a heating-stage comprising of an external furnace (post the mixer stage) where the newly formed particles were effectively heated further (and for longer) in order to improve crystal quality or increase crystal growth or help to increase yields or convert the intermediates to the desired end product..$^{1-72}$ Thereafter, flow processes tended to have a part of the process for cooling to ambient temperatures (e.g. a pipe-in-pipe heat exchanger). In many of these hydrothermal flow systems, it was often reported that particles were collected in in-line filters, or the filters were used to filter out any large particles or agglomerates to protect the back pressure regulator, BPR (a valve that lowers the process slurry pressure back down to atmospheric pressure)..$^{77}$

This mixing of a superheated water and a cold metal salt solution has been suitable to produce a number of different nanomaterials, for example, simple oxides such as $\mathrm{Fe}_{2} \mathrm{O}_{31}{ }^{72,}{ }^{137} \mathrm{CoO}_{1}{ }^{135} \mathrm{Co}_{3} \mathrm{O}_{41}{ }^{72} \mathrm{NiO},{ }^{72,}{ }^{138} \mathrm{ZrO}_{21}{ }^{72} \mathrm{CeO}_{21}{ }^{139}$ as well as mixed oxides such as $\mathrm{Ce}_{x} \mathrm{Zr}_{(1-x)} \mathrm{O}_{2}{ }^{62}$ and $\mathrm{CoFe}_{2} \mathrm{O}_{4}{ }^{135}$ and other compounds such as $\mathrm{La}(\mathrm{OH})_{3}{ }^{140}$ and $\mathrm{AlO}(\mathrm{OH}){ }^{.1}$ In many cases, the early CHFS designs gave low yields (i.e. for $\mathrm{CoO}$ or $\mathrm{ZrO}_{2}$ ) or it was not possible to achieve phase pure materials at reasonable temperatures (i.e. as shown for $\mathrm{Co}_{3} \mathrm{O}_{4}$ and $\mathrm{NiO}$ under certain conditions). ${ }^{136}$ Furthermore, some materials were not accessible at all; for example, $\mathrm{ZnO}$ would not precipitate under neutral conditions. ${ }^{135}$

\section{Figure 3}

Note: The reader is drawn to the fact that, although one of the feeds is supercritical water, after mixing with an ambient temperature metal salt solution (aq.), the resulting mixture may be at considerably lower temperature than the critical temperature of pure water (depending on parameters, as described later). In certain cases (in early publications), one or more precursors underwent premature reactions when their solutions were mixed under ambient conditions, which made it difficult to mix and pump the precursors via standard HPLC pumps. For example, in the synthesis of hydroxyapatite, certain precursor materials $\left(\mathrm{Ca}^{2+}\right.$ and $\mathrm{PO}_{4}{ }^{3-}$ ions) underwent pre-reaction when mixed in the same precursor solution. ${ }^{76}$ The solution to this problem is to add a third pump, which allows two reagents to now be mixed in flow via pumps 2 and 3 (Figure 4a). ${ }^{141}$ 
Figure 4 .

Researchers sometimes used the third pump (figure $4 \mathrm{a}$ ) to pump a base solution $(\mathrm{KOH})$ in order to precipitate the metal salt in situ. The base solution and metal salt feed can be configured, so that they mix either before or after one of the two feeds meets the supercritical water feed. For example, $\mathrm{Co}_{3} \mathrm{O}_{4}$ and $\mathrm{Fe}_{2} \mathrm{O}_{3}$ were found to have a more uniform particle size distribution if the $\mathrm{KOH}$ solution was mixed with the supercritical water feed, before the respective metal salts were added to the mixture in-flow..$^{142}$ In contrast, $\mathrm{ZnO}$ was found to form rounded particles if the $\mathrm{KOH}$ solution was mixed with the supercritical water feed first, or rods if the $\mathrm{Zn}\left(\mathrm{NO}_{3}\right)_{2}$ salt was mixed with the supercritical water feed first. ${ }^{89}$ This arrangement is shown in Figure $4 b^{89}$.

More recently, the third pump was used for pumping reagents other than just $\mathrm{KOH}$ base. For example, $\mathrm{LiOH}$ has been used in this feed in order to produce $\mathrm{LiCoO}_{2}{ }^{20}$ and $\left[\left(\mathrm{NH}_{4}\right)_{2} \mathrm{HPO}_{4}\right]$ has been used as the phosphate source to precipitate hydroxyapatite, $\left[\mathrm{Ca}_{10}\left(\mathrm{PO}_{4}\right)_{6}(\mathrm{OH})_{2}\right]_{1}$ and other doped and undoped calcium phosphates. ${ }^{76}$

In theory, extra HPLC pumps can be added in CHFS systems, but in practice, most inorganic salts will either dissolve in acidic or basic conditions, so this is often unnecessary. However, in the CHFS synthesis of phase pure barium hexaferrite, a four-pump setup was used; the fourth pump was used to provide a flow of heated water $\left(200^{\circ} \mathrm{C}\right)$ that pre-heated the metal salt and base solution mixture before meeting a further flow of superheated water was added (Figure $4 \mathrm{C}) .77$

\subsection{Mixing and Modelling}

In this section, we will briefly review some of the different and novel approaches to mixing, modelling and measurement / imaging that have been used in continuous hydrothermal processes. This is an area, which has given tremendous insights or allowed a better comparison of different mixers and how process conditions, affect the products obtained. Several mixers were developed including off the shelf or customized mixers. A full review of mixers is beyond the scope of this review, therefore, limited space has been devoted to briefly discussing some of the new or interesting designs as a way of introduction to the topic. Poliakoff et al. have also surveyed many mixing designs. ${ }^{143}$

As continuous hydrothermal processes are trying to mix ambient temperature metal salts with supercritical water, it is important to understand that the key aim is to allow rapid mixing of the two feeds (which are at very differ- 
ent temperatures and densities ${ }^{144}$ ), whilst avoiding pre-heating of the metal salts or blockage of the pipe/reactor with products due to fouling. Furthermore, different mixers affect the thermal "history" (time/temperature) that the metal salt (and subsequently the nanoparticles) experience during nucleation and growth. ${ }^{145}$ Thus a full consideration of reactor parameters (flow rates, temperatures, reactions in flow), heat exchange events in the mixer, buoyancy effects versus momentum effects of flows meeting (of the hot water / slurry), changes in properties of the fluids, etc. should be taken into account when choosing mixer designs. Often, a mixer will only work well or as needed within a range of flow rate conditions before it is better to scale-up or scale out a process (i.e. at higher flow rates) or indeed use a different mixer!.

Computational or image analysis ${ }^{144,146}$ techniques can be used to better understand the different mixers in continuous hydrothermal reactors for NP synthesis. Computational fluid dynamics modelling has been used in this regard; in many cases, ${ }^{147}$ it has been carried out retrospectively to explain problems or indeed good mixing behaviors. ${ }^{147^{-156}}$ Other computational studies include the report by Wang et al. who investigated population balance (PB) modelling as a tool to study the effects of solution concentration and reactor residence time, on the particle size distribution. ${ }^{157}$ This is an area which is already giving insights into how process conditions, affect the products obtained.

Historically, many CHFS-systems have favored simple tee-piece mixers (to mix superheated water and metal salts) as the mixers can be assembled from standard off-the-shelf fittings and can be replaced easily and may be available in exotic corrosion resistant materials if needed (albeit at considerable cost). ${ }^{71}$ Tee piece mixers are still very much in use and can operate under a range of flow rates and orientations. ${ }^{30,145,158-160}$ Some recent comprehensive reports on the effects of continuous hydrothermal synthesis operating parameters (Reynolds numbers, flow rates etc.) on ceria NP properties, were reported by Adschiri and co-workers in the temperature range 200 to $380^{\circ}$ C. ${ }^{159}$ In this case, rapid mixing and higher temperatures, favored the formation of smaller NPs. ${ }^{159}$ Furthermore, in order to visualize the mixing behavior of the two streams, Adschiri et al. performed neutron tomography measurements on various mixers ${ }^{161-162}$ for a continuous hydrothermal system at ca. $400{ }^{\circ} \mathrm{C}$ and $25 \mathrm{MPa}$. A three dimensional distribution of neutron attenuation was obtained by rotating the angle of the mixer and collecting images (see Figure 5a) This technique showed itself to be very powerful and could assist researchers in the future in developing not only better mixers, but also finding the optimum conditions for rapid or controlled mixing. 
In comparison to tee mixers, other researchers have reported the use of swirling micro mixers with improved mixing (see Figure $5 \mathrm{~d}$ ). A continuous hydrothermal process incorporating a micro mixer led to a reduction in particle size of bohemite $[\mathrm{Al}(\mathrm{O}) \mathrm{OH}]$ in the range 100 to $200 \mathrm{~nm}$ (for tee mixer) to $60 \mathrm{~nm}$ (by TEM and DLS data). ${ }^{163}$ The same mixer design was also used for $\mathrm{NiO}$ synthesis, with smaller particles again being made compared to a tee mixer. ${ }^{164}$ Other researchers studied tee-piece mixers with non-standard geometries. ${ }^{165-166}$ In a report by Kim et al., continuous hydrothermal synthesis of olivine $\left(\mathrm{LiFePO}_{4}\right) \mathrm{NPs}$ was investigated using three different mixing tee geometries; a $90^{\circ}$ tee (a conventional Swagelok ${ }^{\circledR}$ T-union), a $50^{\circ}$ tee, and a swirling tee. ${ }^{165}$ The use of a swirling tee led to smaller-sized $\mathrm{LiFePO}_{4}$ particles with fewer impurities and better performance. ${ }^{165}$ The disadvantage of these nonstandard designs is that they require custom-made parts, whereas co-current, countercurrent or tee-piece or other mixers can be made quickly and affordably from off-the-shelf, e.g. Swagelok ${ }^{\mathrm{TM}}$ parts.

Lester et al. developed counter-current mixers (called the "nozzle reactor") for CHFS-type processes, ${ }^{167}$ which are able to take advantage of the relative densities of the fluids in the system and mix them without significant preheating of the metal salt feed under certain conditions. ${ }^{152}$ This is a coaxial pipe in pipe counter-current design, arranged vertically such that the hot water enters from the top, the metal salt enters from the bottom and the products are removed upwards (see Figure $5 c$ ). Recently, the authors at UCL (whilst investigating new improved mixing designs) revisited the counter-current mixer and measured the actual temperatures inside the reactor at certain points by placing thermocouples inside various points of the reactor for a wide range of mixing conditions, and particularly the mixing point. The study used pure water for the nominal ambient temperature 'metal' feed (rather than actual solutions with metal salts as materials might build up on the thermocouples placed internally). ${ }^{168}$ This work was extremely revealing and showed that for balanced flowrates (when the flowrates of the superheated water and 'precursors' were equal on a mass basis), the superheated water issuing from the inner pipe, did not penetrate far into the 'precursor' (ambient temperature water) feed. ${ }^{168}$ However, large, rapid fluctuations in temperature with time were observed below the outlet of the inner pipe when the flows were unbalanced (an excess of superheated water) and under such conditions, jetting occurred that was considered to be undesirable for the controlled synthesis of NPs. An increase in temperature of the products as they flowed up through the annulus between the inner and outer pipes, was measured by the authors. Importantly, the data suggested internal transfer of heat between the incoming superheated water (inner pipe) and the cooler products (outer pipe). This meant that the theoretical tem- 
perature of mixing for the products (i.e. the part mixed water feeds in this case) was approached only at the outlet of the mixer. ${ }^{168}$

More recently, the UCL authors developed the highly scalable coaxial or confined jet mixer (see Figure 5b), which offered several advantages including that the mixing between the ambient temperature and sc-water feed occurs almost instantly. Furthermore, the sc-water feed can run at higher flow rates to achieve very high mixing temperatures of $370^{\circ} \mathrm{C}$ or higher (known as unbalanced mixing). ${ }^{148,169-170}$ This mixer has been shown to make very similar materials when scaling up from lab to pilot plant CHFS process ( $>2 \mathrm{~kg} \cdot \mathrm{h}^{-1}$ by dry mass), as will be discussed later. ${ }^{170}$ Furthermore, two mixers can be placed in series to allow nucleation in the first mixer (at a higher temperature) and then the second mixer can be used to add capping agents or quenching water into the process ${ }^{171}$ (this approach with two mixers in series has also been studied with other mixer designs such as tee-piece). For example, Iversen and Aymonier have also favored the use of co-flow mixers for the synthesis of well-defined nanoparticles. ${ }^{172}$ The same researchers have performed a wealth of in situ hydrothermal / solvothermal semi-batch (rapid heat) as well as continuous process studies using in situ X-rays to better understand nucleation and growth kinetics and changes in particle crystallinity and phase evolution in the first few seconds under supercritical conditions. ${ }^{173-180}$ This work has been tremendously useful in trying to evaluate the effects of residence times and conditions on particle evolution under extreme conditions.

Figure 5 
4 OPTICAL APPLICATIONS (NON-BIO USE)

Optical applications for nanomaterials are very much of interest given the rise of nanomaterials in electro-optical printed devices and the ability to tune colour with the size of certain nanomaterials. ${ }^{1,}{ }^{181}$ Other materials include high-density laser lens materials where the use of nanomaterials may allow the densification process to be performed under relatively milder conditions compared to more traditional solid state reactions. Another emerging area for nanomaterials with useful optical properties is in biolabels for cell imaging or therapies; these applications will be covered later in the biomedical applications. ${ }^{182}$

\subsection{Nanophosphors (Non-Bio Uses)}

Inorganic phosphors are typically transition metal compounds (often with rare earth elements) that phosphoresce. The most common uses of phosphors are in cathode ray tube (CRT) displays and fluorescent lights. Phosphors are used in field emission displays (FED) and plasma displays (PDP). FEDs and PDPs have lower energy requirements and are generally more compact than a CRT screen. FEDs use a high current density, which can cause deterioration of the phosphor powder. In PDPs high energy photons impinge on the phosphor. The phosphor requires high thermal stability and a long lifetime. ${ }^{141,183}$ One of the potential advantages of using CHFS type processes for the fabrication of phosphors, include that it may be possible to make some materials directly in a single step or that such materials may sinter at lower temperatures (e.g. for laser lenses) than are more conventionally used.

Yttrium Aluminium Garnet Based Materials. Yttrium Aluminium Garnet ( $\mathrm{YAG}$ or $\mathrm{Y}_{3} \mathrm{Al}_{5} \mathrm{O}_{12}$ ) is an important phosphor material. It possesses a high efficiency of energy transfer and is resistant to radiation damage, which makes it useful in laser lenses; because it is usable in the UV to mid IR range of the electromagnetic spectrum, it is useful for IR and laser windows. YAG also has a cubic crystal structure, which gives it optical isotropy (unlike sapphire). It is used as a host material for full colour phosphors by doping with lanthanide ions. ${ }^{184-185}$ Furthermore, YAG based phosphors are used for cathode ray tube applications because of their thermal stability and resistance to saturation at high current excitations ${ }^{183}$

Conventionally YAG is prepared via a solid-state reaction of yttrium oxide and alumina in the range 1600 to 1800 ${ }^{\circ} \mathrm{C}$. The process is slow and reaction intermediates such as $\mathrm{YAIO}_{3}(\mathrm{YAP})$ and $\mathrm{Y}_{4} \mathrm{Al}_{2} \mathrm{O}_{7}(\mathrm{YAM})$ form and only transform into YAG at high temperatures. ${ }^{186}$ Wet chemical routes such as sol-gel, ${ }^{187}$ co-precipitation, ${ }^{184,}{ }^{188}$ combustion, ${ }^{189}$ 
and spray pyrolysis, ${ }^{190-191}$ have been used to synthesise YAG phosphors. Additional heat-treatments steps are also often required (e.g. sol-gel and co-precipitation) and aggregation of products during post heat-treatment, is common. ${ }^{192}$ Other methods, e.g. spray pyrolysis and combustion, can also lead to large particles and a high degree of aggregation. ${ }^{190}$ Batch hydrothermal methods have also been used to manufacture YAG NPs (including surface functionalised ones ${ }^{193}$ ) and in some cases, this required an initial precipitation followed by a hydrothermal autoclave reaction step. ${ }^{194}$

Continuous hydrothermal methods have been used to make phosphors ${ }^{52,195-197}$ which include YAG, 94, 198-199 europium doped YAG (YAG:Eü $\left.{ }^{3+}\right)$ and terbium doped YAG (YAG:Tb)94, 141, 183, 196 and co-doped $\mathrm{Ce}^{3^{+}} / \mathrm{Eu}^{3^{+}}$YAG. $^{200}$

Figure 6.

Hakuta et al. synthesized YAG:Tb at $400{ }^{\circ} \mathrm{C}$ in a flow reactor using an aqueous solution of metal salt solution containing $\mathrm{Al}^{3+}, \mathrm{Y}^{3+}$ and $\mathrm{Tb}^{3+}$ ions $\left[\right.$ from $\mathrm{Al}\left(\mathrm{NO}_{3}\right)_{3} \cdot{ }_{9} \mathrm{H}_{2} \mathrm{O}, \mathrm{Y}\left(\mathrm{NO}_{3}\right)_{3} \cdot 6 \mathrm{H}_{2} \mathrm{O}$ and $\mathrm{TbCl}_{3} \cdot \mathrm{nH}_{2} \mathrm{O}$, and aqueous $\mathrm{KOH}$ that were pumped separately. ${ }^{141}$ The reactor temperature was maintained at ca. $400{ }^{\circ} \mathrm{C}$ with flow rates of $2 \mathrm{~mL} \cdot \mathrm{min}^{-1}$ for the metal ion and $\mathrm{KOH}$ feeds, respectively, and $8 \mathrm{~mL} \cdot \mathrm{min}^{-1}$ for the water, the pressure in the reactor was maintained at $30 \mathrm{MPa}$. Single phase $\mathrm{YAG}$ was produced at $400^{\circ} \mathrm{C}$ (and $30 \mathrm{MPa}$ ) with a stoichiometric $(\mathrm{Y}+\mathrm{Tb}) / \mathrm{Al}$ ratio $[(\mathrm{Y}+\mathrm{Tb}) / \mathrm{Al}$ $=0.6]$. The particles appeared to be single crystals and showed the characteristic emission of a YAG:Tb phosphor, with a comparable maximum intensity to that of a material produced through a solid-state reaction. Excess $\mathrm{Al}^{3^{+}}$ions produced additional $\mathrm{AlOOH}$ phases, and excess $\mathrm{Y}^{3+}$ ions produced $\mathrm{YOOH}$ phases under these conditions. At higher alkali molar ratios, $\mathrm{YOOH}$ formed with the YAG (and because of a low conversion of Al). At low alkali molar ratios, AlOOH formed due to a low conversion of $\mathrm{Y}$ and $\mathrm{Tb} \cdot{ }^{141}$

Temperature also had an effect on the continuous hydrothermal synthesis of single phase YAG. ${ }^{141}$ Temperatures higher than the critical point of water, led to higher conversions and in the range 350 to $420^{\circ} \mathrm{C}$ (because the selectivity of YAG increased with increasing temperature). Below the critical point, yttria ions were too soluble and thus, conversion rates were low (ca. $16 \%$ ) and, therefore, $\mathrm{Al}(\mathrm{O}) \mathrm{OH}$ was formed. In a later paper by Hakuta et al., ${ }^{196}$ it was found that YAG was produced only when the $\mathrm{pH}$ of the starting solution was ca. 9. At neutral $\mathrm{pH}, \mathrm{AlOOH}$ was also produced and at $\mathrm{pH}>$ 9, YAG and $\mathrm{YOOH}$ were both produced. In the formation of YAG:Eu ${ }^{3+}$, residence time was 
found to play an important role in the formation of the YAG and for long residence times (i.e. 20 s), crystalline YAG could be formed at any $\mathrm{pH}^{183}$

A number of phosphors have also been made using water and alcohol or organic mixtures as the solvents. ${ }^{44}$ Clearly, though the use of alcohols in part for water is generally less desirable/sustainable than using water alone, but as will be seen later, the use of organic co-solvents appears to favour enhanced reaction rates or alters particle size or improves crystallinity for some NPs made in flow. ${ }^{94}$ For example, Crystalline YAG particles were produced in a continuous hydrothermal process using precursor solutions containing $\mathrm{Y}^{3^{+}}$and $\mathrm{Al}^{3+}$ salts in water-ethanol mixtures.94 The YAG was produced in a single step and higher $\mathrm{pH}$ tended to favour nucleation as opposed to growth, which led to smaller particles; this can be explained by reaction equilibrium theory. ${ }^{201}$ The reaction conditions used to make YAG in water/ethanol mixtures were in the temperature range 260 to $385^{\circ} \mathrm{C}$ and $24 \mathrm{MPa}$. The flow rate for the $\mathrm{scH}_{2} \mathrm{O}$ feeds was in the range 10 to $20 \mathrm{~mL}$. $\mathrm{min}^{-1}$ and the precursor solution feed was half that of the $\mathrm{scH}_{2} \mathrm{O}$. The ratio of ethanol:water in the feed solution affected the particle size; particles produced from a $60 \%$ ethanol precursor solution, were smaller (ca. $50 \mathrm{~nm}$ ) and less crystalline than those produced from $90 \%$ ethanol precursor solutions. It is interesting to note that the authors used water / alcohol mixtures to affect the crystallinity of the final products, which potentially offers the ability to tune the reaction chemistry. Indeed Hoffmann and colleagues ${ }^{202}$ suggested that the degree of $\mathrm{H}$-bonding in sc-alcohols can be tuned as a function of density. Furthermore, as alcohols have lower critical temperatures that water (e.g., for scMeOH, $\mathrm{T}_{\mathrm{c}}=240^{\circ} \mathrm{C} / \mathrm{P}_{\mathrm{c}}=81 \mathrm{bar}{ }^{28}$ ), which means that hydrogen bonding largely breaks down at much lower temperatures than in sc-water. Therefore, when such alcohols are combined with water, they may be inclined to display different effects on nucleation, growth and crystallisation for nanoparticles in flow compared to supercritical water alone. ${ }^{203}$ Indeed, in the aforementioned example for YAG94, when more alcohol was present, particles tended to be more crystalline.

In comparison, Chudoba et al. found that favorable conditions for single step YAG production were achieved using acetates (and acetylacetonates) in aqueous-alcoholic solutions as starting materials. Aqueous and alcoholic solutions of nitrates and aqueous solutions of acetates were found not appropriate as precursors for YAG production; after annealing the as-prepared powders at $600^{\circ} \mathrm{C}$, comparable density was obtained to a material made via a more conventional precipitation and heat-treatment route. ${ }^{199}$

In a report by Lee et al., YAG co-doped $\mathrm{Ce}^{3+} / \mathrm{Eu}^{3+}$ was made in continuous hydrothermal process via the reaction of a mixture of base and metal salts with supercritical water. ${ }^{200}$ For a reaction time of $20 \mathrm{~s}$ and at a pH of 9.1 , the 
average size of NPs was in the range 60 to $150 \mathrm{~nm}$ (mixture of cubic or hexagonal particles co-existing with spherical particles). The as-made nanophosphors, had a broad emission (green-yellow region) due to $\mathrm{Ce}^{3+}$ and a sharp emission centred in the red (at $610 \mathrm{~nm}$ due to $\left.\mathrm{Eu}^{3^{+}}\right) .{ }^{200}$ This report revealed no secondary phases present under certain conditions using $\mathrm{KOH}$, which is in contrast to many other reports on the attempted synthesis of YAG.

More recently, $\mathrm{Y}_{3} \mathrm{Al}_{5} \mathrm{O}_{12}(\mathrm{YAG})$ and $\mathrm{Yb}_{3} \mathrm{Al}_{5} \mathrm{O}_{12}(\mathrm{YbAG})$ were synthesized from "gels" in a flow system. ${ }^{198}$ In the report, a white gelatinous precipitate (from the corresponding metal nitrates) was first produced, which was suspended in deionized water, before the slurry was fed into a custom-made continuous flow reactor with a heated chamber. ${ }^{198}$ Structural changes of the garnet structures were then followed using variable temperature measurements on the dried products using powder X-ray diffraction at a synchrotron source in the temperature range 100 to $1000 \mathrm{~K} .{ }^{198}$

Zinc Based Phosphors. ZnO is also a promising phosphor material. It has a significant green emission at $510 \mathrm{~nm}$ (excitation $350 \mathrm{~nm}$ ) due to singly ionized oxygen vacancies ${ }^{204-205}$. $\mathrm{ZnO}$ has a large exciton binding energy (6o MeV) ${ }^{206}$ and has UV emission at $380 \mathrm{~nm}$ due to exciton emission ${ }^{207}$. ZnO has the same crystal structure as GaN-based compounds, but it is more stable in comparison. It is therefore, thought that the degradation of emission properties due to defect propagation, may not be such a problem for $\mathrm{ZnO}$ materials. ${ }^{208}$ Hence, $\mathrm{ZnO}$ can be used as a high efficiency, low voltage phosphor. ${ }^{209}$

Nano-ZnO can be made reliably and efficiently in a CHFS with a large degree of size and some shape control. $\mathrm{ZnO}$ has been reported to show red emission $(620 \mathrm{~nm})^{208}$ which may have been due to trace elements or doubly ionised oxygen vacancies. ${ }^{210}$ High crystallinity is evidently important for photoluminescence and emission efficiency. In this case, the sharp XRD peaks suggest that despite the small size, the CHFS-process produced ZnO had high crystallinity (suggesting high efficiency and low emission threshold due to quantum effects associated with nano-size).

ZnO can also be used for UV lasing applications as the high exciton binding energy gives it a low UV lasing threshold (nano-ZnO has an even lower lasing threshold due to quantum effects). ${ }^{206}$ Literature reported photoluminescence $(\mathrm{PL})$ spectra of $\mathrm{ZnO}$ have previously shown strong near band-gap edge emission ( $380 \mathrm{~nm}$ ) and thus, optimization of CHFS-process conditions can give high UV emission efficiency. ${ }^{208}$ The authors reported the formation of a green $\mathrm{ZnO}$ phosphor when they attempted to prepare sulfur-doped $\mathrm{ZnO}$, which instead produced a partially reduced $\mathrm{ZnO}$ with the typical wurtzite structure. 
Han et al. examined the optical properties of small (size range 10 to $15 \mathrm{~nm}$ ) $\mathrm{ZnO}$ NPs synthesized in scMeOH and $\mathrm{scH}_{2} \mathrm{O}$, respectively. ${ }^{211}$ They also obtained surface functionalization with oleic acid and suggested that the photoluminescence (PL) spectra and photophysics, were strongly affected by the presence of the coordinating ligands. In the absence of oleic acid, ZnO NPs with a mean diameter of $\sim 15 \mathrm{~nm}$ (that formed secondary aggregates of $\sim 150 \mathrm{~nm}$ in size), were shown to possess interstitial zinc and oxygen vacancies as the major defects. In contrast, the oleic acid capped particles appeared to have significantly reduced zinc interstitials. ${ }^{211}$

Aymonier et al. demonstrated the synthesis of well-defined excitonic luminescent $\mathrm{ZnO}$ nanoparticles (from $\mathrm{Zn}(\mathrm{acac})_{2} \cdot \mathrm{H}_{2} \mathrm{O}$ and trioctylphosphine in the presence of $\mathrm{H}_{2} \mathrm{O}_{2}$ in supercritical alcohol at $250{ }^{\circ} \mathrm{C} / 25 \mathrm{MPa}$ ) using the carefully controlled microfluidic flow system, using a horizontal co-flow mixer (co-current, pipe-in-pipe type). ${ }^{212}$ Due to the limited production rates of the microscale approach, the same team investigated the influence of scale-up (up to $100 \mathrm{mg} \cdot \mathrm{h}^{-1}$ ) from micro to milli-fluidic systems on the optical properties of the $\mathrm{ZnO}$ nanocrystals. They demonstrated a number of different shapes for $\mathrm{ZnO}$ depending on the process set-up. Furthermore, all of the nanocrystals revealed a strong excitonic-only emission in the UV region in the room temperature photoluminescence $(\mathrm{PL}){ }^{212}$ This report clearly highlights the potential benefits of going to smaller scale flow processes for supercritical hydrothermal processes, which may offer some advantages despite the relatively low production rates. The future directions for such materials will no doubt lie in their use for novel optical advanced materials for displays, lasers, lenses, optochemical sensors, novel security inks or for biological imaging (see later section 5.5), particularly their use in non-oxides based chemistries (e.g. sulfides, nitrides, oxy-nitrides) where tuneable emission and excitation may be possible. Indeed, Tang et al. reported the synthesis of ZnS uncoated particles in, which surface agents were seen to alter its photoluminescent properties (see more information on this material in section $5 \cdot 5$ ) ${ }^{213}$

Other Phosphor Host Materials and Considerations. As well as YAG based materials, there have been a number of reports on the CHFS synthesis of phosphors based on yttrium oxide (or the hydroxide or oxyhydroxide), zirconia $^{214}$ or silicates ${ }^{215}$ or other hosts lattices doped with rare earths such as Eu or Tb, or Pr doped Perovskites..$^{216}$ Lester et al. reported the synthesis and properties of Eu doped $\mathrm{ZrO}_{2}{ }_{1}^{214}$ as well as Eu doped yttria ${ }^{217}$ (evaluated for biological applications as will be seen later in section $5 \cdot 5$ ).

Pr-doped $\mathrm{CaTiO}_{3}$ nanoparticle phosphors (strong red emission peak at $613 \mathrm{~nm}$ ) were made in flow at $400{ }^{\circ} \mathrm{C}$ and $30 \mathrm{MPa}$ from $\mathrm{Pr}$ and $\mathrm{Ca}$ nitrates and $\mathrm{T} \mathrm{TiO}_{2}$ sol $(5 \mathrm{~nm})$, and aqueous solutions of $\mathrm{KOH}^{216}$ A T-type micromixer was 
used for rapid heating of the aqueous solutions ( $22 \mathrm{~nm}$ particles were made at a residence time of $5.0 \mathrm{~s}$ ). In particular, the mechanisms of product formation were better understood by looking at the effects of residence time and $\mathrm{KOH}$ molality on the Ca:Ti ratio, particle, diameter, crystallite diameter, and crystal structure. ${ }^{216}$ With increasing residence time and also $[\mathrm{KOH}]$ the Ca:Ti ratio in the solid increased up to a value of $1: 1$, resulting in $\mathrm{CaTiO}_{3}$ (orthorhombic) as the major phase. A formation mechanism is proposed in which dissolution of the $\mathrm{TiO}_{2}$ sol, led to formation of a $\mathrm{Ca}: \mathrm{Ti}$ hydroxide precursor though hydrolysis, then nucleation-growth of $\mathrm{CaTiO}_{3}$, via dehydration / condensation. ${ }^{216}$

Defects in crystals can arise during the synthesis of ceramic phosphors from component chemicals. These defects can reduce luminescence intensities, however, if lower temperature synthesis and crystallisation methods to manufacture phosphors can be developed, it may be possible to avoid having to heat phosphor materials as high as $1000^{\circ} \mathrm{C}$. Indeed, some of the authors manufactured nanophosphor libraries (using a robotic CHFS system) in order to study the effects of heat-treatment temperatures as well as the dopant concentration on luminescence of the as as-made NPs. ${ }^{218}$ Nanocrystals were synthesized in CHFS from precursor solutions and eight unique compositions comprising pure as-prepared nano-Y $(\mathrm{OH})_{3}$ and several Eu ${ }^{3+}$ doped-yttrium hydroxide nanocrystal samples. The $\mathrm{Y}(\mathrm{OH})_{3}: \mathrm{Eu}^{3+}$ samples were split up and then manually placed in a furnace and heat-treated in air for $4 \mathrm{~h}$ in the temperature range 200 to $1200{ }^{\circ} \mathrm{C}$ [at $100{ }^{\circ} \mathrm{C}$ intervals, giving a total of 84 samples plus one as-prepared ${ }^{28}$ pure $\mathrm{Y}(\mathrm{OH})_{3}$ sample]. ${ }^{218}$ The as-prepared and heat-treated ceramic samples were affixed in an aluminium well plate holder and analysed using a fluorescence spectrometer (illuminated with a $254 \mathrm{~nm}$ light). The $3 \mathrm{~mol} \% \mathrm{Eu}^{3^{+}}$sample heat-treated at $1200{ }^{\circ} \mathrm{C}$ formed $\mathrm{Y}_{2} \mathrm{O}_{3}: \mathrm{Eu}^{3+}$ and gave the most intense fluorescence overall (major red peak at $612 \mathrm{~nm}$ ). However, an identical nanocrystal sample heat-treated at only $500{ }^{\circ} \mathrm{C}$ (also identified as $\mathrm{Y}_{2} \mathrm{O}_{3}: \mathrm{Eu}^{3+}$ after this heat-treatment) was the brightest phosphor under illumination of the samples heat-treated at or below $1000^{\circ} \mathrm{C} .{ }^{218}$ Evidently, these conditions were ideal for the conversion to the oxide with relatively few defects, which promoted the locally high luminescence.

Figure 7 .

Hayashi and co-workers reported that Eu ${ }^{3+}$ doped yttria NPs could be synthesized by continuous hydrothermal process using the respective metal nitrate reagents and $\mathrm{KOH}$ solution, heated at in the temperature range 350 to 
$450^{\circ} \mathrm{C}$ (pressure $30 \mathrm{MPa}$ ), and followed by a further heat-treatment. Powder XRD data for the as-prepared products, confirmed that (doped) $\mathrm{YOOH}$ was made; this could be converted into cubic-phase $\mathrm{Y}_{2} \mathrm{O}_{3}$ after an additional heat-treatment step at $>550^{\circ} \mathrm{C}$. Notably, the particle size was also $<50 \mathrm{~nm}$ after heat-treatment (at as high as $\left.800^{\circ} \mathrm{C}\right)$. Effects of $\mathrm{CHFS}$ process reaction time, post synthesis annealing temperature and amount of Eu $3^{+}$dopant on photoluminescence intensity, were examined. The as-prepared particles exhibited red emission without annealing at high synthesis temperatures, whereas photoluminescence intensity (at $612 \mathrm{~nm}$ ) generally increased with higher annealing temperature (post synthesis). Notably, other researchers have tried to make $\mathrm{Y}_{2} \mathrm{O}_{3}$ (as well as $\mathrm{La}_{2} \mathrm{O}_{3}$ ) directly in a CHFS-type process, but were largely unsuccessful, instead often obtaining $\mathrm{Y}(\mathrm{OH})_{3}\left[\mathrm{or} \mathrm{La}(\mathrm{OH})_{3}\right]$, which then needed to heat-treat for $2 \mathrm{~h}$ to form the oxide at $773 \mathrm{~K}\left[\right.$ for $\left.\mathrm{Y}(\mathrm{OH})_{3}\right]$ or $1073 \mathrm{~K}\left[\right.$ for $\left.\mathrm{La}(\mathrm{OH})_{3}\right]$ for $2 \mathrm{~h} .{ }^{219}$

There are relatively few reports of silicate type phosphor materials in the CHFS literature, therefore, one of the more unusual reports relating to phosphors ${ }^{126}$ was on the synthesis of sub-micron $\mathrm{Zn}_{2} \mathrm{SiO}_{4}: \mathrm{Mn}^{2+}$ particles, where colloidal silica (or sodium silicate) was used as the Si source. ${ }^{126,220}$ The reactions required high temperatures and pressures (temperature range 400 to $500{ }^{\circ} \mathrm{C}$, at pressures ranging from 30 to $35 \mathrm{MPa}$ ) using $\mathrm{NaOH}$ as a base. It was found that when sodium silicate was used, $\alpha$ - and $\beta$ - doped zinc silicate phases were formed regardless of the $\mathrm{Zn}$ and Mn sources. ${ }^{126}$ However, when colloidal silica was used, the products were more sensitive to the $\mathrm{Zn}$ and $\mathrm{Mn}$ precursors used or the use of base. As the reaction temperature increased, the crystallinity of $\alpha$-phase increased and substitution of the $\mathrm{Mn}^{2+}$ into the $\mathrm{Zn}$ sites was shown to decrease. The $\alpha-\mathrm{Zn}_{2} \mathrm{SiO}_{4}: \mathrm{Mn}^{2+}$ particles were round in shape, having an average diameter of ca. $268 \mathrm{~nm}$, with a green-emission (wavelength of $524 \mathrm{~nm}$ ). ${ }^{126}$

\subsection{UV Attenuating Nanoparticles}

Even though a minimal level of solar radiation is considered good for living organisms, ${ }^{221}$ such as encouraging vitamin D production in humans, ${ }^{222} \mathrm{UV}$ light is more energetic than visible light and it can cause damage to human and animal skin, ${ }^{223-227}$ degradation of polymers, ${ }^{228-230}$ bleaching and fading of dyes and paints ${ }^{231-233}$ and reduce the shelf life of foods such as fresh meat and vegetables. ${ }^{234-236}$

The effect of UV on humans was discovered as far back as 1798 when Willan described sensitivity to light under the term 'eczema solare'. ${ }^{237}$ Since then, UV radiation has been found to cause acute and chronic problems to humans after prolonged exposure such as sunburn and premature aging. It can also cause breakage in DNA strands and mutagenesis of p53 cells and damage to chromosomes, leading to tumors $226-227$ and skin cancer. In food, UV 
light also acts as a catalyst for oxidation leading to spoilage and discolouration. ${ }^{234,}{ }^{238} \mathrm{UV}$ free lighting in food displays has significantly extend shelf life of pre-packaged meats. ${ }^{236,239-240}$ UV radiation can cause photodegredation in polymers and it can be a contributing factor for premature failure in plastic components. ${ }^{228}$ UV radiation generates free radicals, which propagate within a polymer and lead to chemical modification and a reduction of chain length. This can lead to reduction in the useful mechanical properties of the polymer $241-24^{2}$ including a loss of transparency, and yellowing. The degradation is mostly at the surface of a polymer, leading to a brittle surface layer and a fragile product. ${ }^{241}$

Inorganic filters are determined by their absorption and scattering properties ${ }^{17}$ and in cosmetic formulations, by their cosmetic acceptability. ${ }^{243} \mathrm{ZnO}$ and $\mathrm{TiO}_{2}$ are widely used as UV absorbers and are the only inorganic UV absorbers allowed for use in human cosmetic applications by the Scientific Committee on Cosmetic and Non-Food Products Intended for Consumers (Europe) ${ }^{244}$ and the food and drug administration (FDA). ZnO and titania are also used as polymer additives and coatings for packaging materials, fabrics and paints. ${ }^{242,245-257}$

Figure 8

Pigmentary $\mathrm{TiO}_{2}$ and $\mathrm{ZnO}$ scatter visible and UV light and appear brilliant white, which makes them aesthetically unsuitable for sunscreens. ${ }^{17}$ Mie scattering predicts that the optimum practical size of titania for light scattering is in the range 180 to $220 \mathrm{~nm}^{258}$ but at this particle size, $\mathrm{TiO}_{2}$ is visible on the skin and is therefore, not transparent or cosmetically desirable ${ }^{259}$. Reducing the particle size of $\mathrm{TiO}_{2}$ or $\mathrm{ZnO}$, reduces the amount of scattering in the visible range and below ca. $50 \mathrm{~nm}$, particles of zinc oxide and titania become transparent on the skin. ${ }^{17,}{ }^{258}$ Nanosized titania and $\mathrm{ZnO}$ absorb UV radiation as below $385 \mathrm{~nm}$ (anatase) or $400 \mathrm{~nm}$ (rutile, $\mathrm{ZnO}$ ), but do not scatter visible light. These micro or ultrafine ceramics are more widely used for cosmetic, coating and packaging applications as they are transparent and therefore, aesthetically more suitable. ${ }^{17}$

$\mathrm{ZnO}$ and titania can also be made in a continuous hydrothermal system for photocatalyst applications (or conversely UV attenuating applications for poor photocatalysts). ${ }^{72,} 87,89,170,197,260-264,270$ The properties of $\mathrm{ZnO}$ can be modified by changing the concentration and flow rates of the metal ion feed, ${ }^{89,265}$ changing the reaction conditions for example by adding base. ${ }^{87}$ The effect of changing the mixing conditions and solubility of $\mathrm{ZnO}$ at the reaction point, can be seen. ${ }^{89}$ The particle size of $\mathrm{ZnO}$ made in CHFS methods, typically ranges from 10 to $300 \mathrm{~nm}$ depending 
on the precursor and conditions. It is known from batch hydrothermal syntheses of $\mathrm{ZnO}$ that additives can be used to control shape and aspect ratio as well as hierarchical structures. ${ }^{120,266}$ Similarly in hydrothermal flow, morphology can easily be changed depending on the mixing methods and additives such as $\mathrm{H}_{2} \mathrm{O}_{2} \cdot{ }^{120,} 208,263,267$

In 2011, Iversen et al. reported a fundamental study into the size control of $\mathrm{ZnO}$ under a range of fast flow conditions and found that anisotropic particles up to $1 \mu \mathrm{m}$ were produced at $122^{\circ} \mathrm{C}$ in flow, whilst $25 \mathrm{~nm}$ rhombic particles we obtained at temperatures $>200^{\circ} \mathrm{C}$. It was observed that variation of the pH gave different shapes; (i) acidic conditions produced large rods, (ii) neutral conditions gave isotropic rhombic particles, and (iii) alkaline conditions resulted in plates. Finally, particle size increased with the precursor concentration. The size and morphology tunability of ZnO made in flow, makes it suitable for UV attenuation applications such as coatings, films and cosmetic applications. ${ }^{268}$ In contrast to $\mathrm{ZnO}$, nano-titania made via CHFS, typically affords particles in the size range 5 to 20 $\mathrm{nm},{ }^{72,219,269}$ which is also potentially suitable for (optically transparent) UV attenuation applications. ${ }^{270}$

Recently, a pilot plant scale continuous Hydrothermal synthesis process was used to control the crystallite size (and surface area) for nano- $\mathrm{TiO}_{2}$ in the range ca. 5 to $18 \mathrm{~nm} .{ }^{271}$ In the report, titanium oxysulfate (and boric acid) were mixed in flow with a feed of potassium hydroxide solution (at $24.1 \mathrm{MPa}$ ), and then this combined stream was mixed with a flow of superheated water (at $400^{\circ} \mathrm{C}$ and $24.1 \mathrm{MPa}$ ) in a confined jet mixer. Nano- $\mathrm{TiO}_{2}$ particles were formed instantly and then cooled inline before being collected as a slurry from the exit of the process. It was observed that the boric acid concentration in the precursor feed, affected reaction $\mathrm{pH}$, which in turn, determined the average crystallite size of the nano- $\mathrm{TiO}_{2}$ as the $\mathrm{pH}$ increased, larger crystallites were obtained. The photoactivity of the nanomaterials were evaluated in a sacrificial photocatalytic water splitting system (hydrogen evolution), and it was found that smaller $\mathrm{TiO}_{2}$ prepared under mildly acidic conditions, yielded the highest photoactivity. ${ }^{32}$

In 2016, some of the authors significantly improved the photocatalytic properties of NP-titania using a multistep process. ${ }^{272}$ First, ultrafine citrate coated $\mathrm{TiO}_{2}$ nano-crystallites (at ca. $5 \mathrm{~nm}$ ) were made in hydrothermal flow and then heat treated at $\mathrm{ca} .300^{\circ} \mathrm{C}$ for $2 \mathrm{~h}$ under a $\mathrm{N}_{2}$ atmosphere to form titania-carbon interfaces (due to the anoxic conditions). The heat-treated sample possessed a highly distorted crystal lattice (with surface oxygen vacancies and $\mathrm{Ti}^{3+}$ species in the bulk). UV/Vis, photoluminesence ( $\mathrm{PL}$ ) and transient photocurrent (TP) measurements, revealed that oxygen vacancies and formation of $\mathrm{C}$ heterojunctions, enhanced visible light absorption and promoted electron-hole pair separation, which led to enhanced photocatalytic hydrogen production (water splitting) being ca. 40 to 50 -fold higher activity than the commercial standard mixture of anatase and rutile titania, Degussa $\mathrm{P} 25 .{ }^{272}$ 
The photoreactivity of pure $\mathrm{TiO}_{2}$ and $\mathrm{ZnO}$, respectively, can limit their usefulness as UV protective agents, despite the fact that they remain largely unaffected by UV radiation like most organic UV filters ${ }^{273}$. The photoelectrons and holes generated on the absorption of UV radiation, can migrate to the surface and react with surface adsorbed species such as water to produce reactive species such as hydroxyl radicals, which can cause degradation in polymers and damage to skin and DNA $243,274^{-275}$. The photoactivity of $\mathrm{TiO}_{2}$ and $\mathrm{ZnO}$ therefore, needs to be diminished for UV protection applications. This can be achieved by coating ${ }^{276-277}$ NPs or doping them..$^{273}$ Doping can easily be accomplished in a CHFS system by adding the appropriate metal salt to the precursor feed in the desired ratio. ${ }^{267,} 278$ Doping with ions such as $\mathrm{Fe}^{3+}$ and $\mathrm{V}^{4^{+}}$has been found to reduce the photocatalytic activity of titania significantly when it is doped in this manner.

CHFS was used by the authors to make mixed Zn-Ti oxides (i.e. a range of anatase titanias doped with zinc oxide and zinc oxides (wurtzite) doped with titania as well as mixtures. ${ }^{279}$ The UV/Vis absorption profiles for the Ti-Zn-O binary mixtures, revealed two main shapes. Firstly, a $\mathrm{ZnO}$ type profile (with a characteristic steep absorption edge and a flat absorption above and below the bandgap (shown as sample 13 in Figure 9, which is pure ZnO). Secondly, a $\mathrm{TiO}_{2}$ type profile was observed, where the bandgap transition was less steep and varied over a wider wavelength range (sample 1, which was pure anatase $\mathrm{TiO}_{2}$ ). For the doped samples and phase mixtures, as prepared samples with high Ti content (samples 2 to 6 ), were similar to the $\mathrm{TiO}_{2} \mathrm{UV} / \mathrm{Vis}$ absorption profile, whereas, the samples with higher Zn ratios (samples 8 to 12), tended to have ZnO like UV/Vis absorption profiles.

Figure 9:

On the other hand, when the photoactivity of a series of doped $\mathrm{ZnO}$ samples was evaluated, Ce doping suppressed the activity of ZnO. Therefore, this material may be a useful UV-attenuator material. ${ }^{267}$ Therefore, similar to the case for the development of photoactive materials, the area of UV attenuating NPs is set to continue and researchers should look at rapidly making and testing doped materials at sub $50 \mathrm{~nm}$ in the future. This will afford a combination of both good UV protection (particularly in the higher energy UVC end too) and optical transparency, as well as good dispersability in appropriate solvents / oils where possible.

\subsection{Refractive Index Modifiers}


The Refractive Index (RI) of a material is a measure of how light propagates through it relative to a vacuum. ${ }^{280}$ The RI of a polymer is based on several factors, which include structure, chain flexibility, molecular geometry, polarizability, and the polymer backbone orientation. The RI of a polymer is a key property for advanced optical devices that include functional materials for lenses, anti-reflective coatings, coatings for organic LEDs and ophthalmic applications. All these devices need high Rls because it allows the devices to be made thinner and more transparent. ${ }^{280}$ High values for RI (>1.50) can be achieved by the inclusion of transparent additives. ${ }^{280}$

A number of materials are known to act as optical (refractive index) modifiers, e.g. $\mathrm{TiO}_{2}, \mathrm{ZrO}_{2}$ and $\mathrm{SiO}_{2}$ to name a few. ${ }^{281}$ For example, zirconia possesses a unique combination of chemical and physical properties, including high refractive index, high transparency (range UV to infrared), ${ }^{281}$ high dielectric constant, high hardness, and high chemical inertness, allowing for uses in a broad array of applications in polymers or paints. ${ }^{231-232,282}$ These nanomaterials can also affect the mechanical properties of the final materials such as improving abrasion or scratch resistance as well as increasing modulus. ${ }^{231-232,282}$

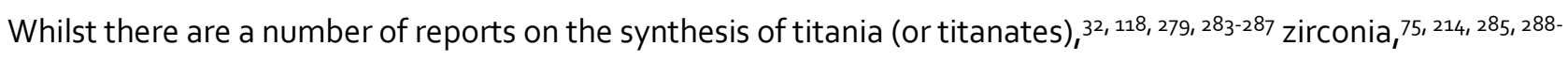
${ }^{289}$ there are no published reports to our knowledge of continuous hydrothermal synthesis made materials being evaluated as a dispersion to alter refractive indices, as such work is most likely highly commercial in nature. However, the authors would suggest that continuous hydrothermal processes are ideal for the manufacture of such materials because they can be surface functionalized. This allows them to be dispersed in polymers / solvents and particles can be made sufficiently small (at least $<50 \mathrm{~nm}$ ) so that they appear transparent / translucent rather than opaque in dispersions / polymers, whilst altering the refractive index of the host. Indeed there are reports in short term batch hydrothermal experiments ${ }^{290}$ that suggest surface functionalized $\mathrm{ZrO}_{2}$ or titania should be readily accessible in flow. Furthermore, Adschiri et al. reported the batch synthesis of $\mathrm{SFN} \mathrm{TiO}_{2}$ (anatase) with a phosphonic acid surface modifier that was very strong bound, resulting in extraordinarily high chemical and thermal stability. ${ }^{44}$

\subsection{Non-linear optics}

Nonlinear optics (NLO) is the branch of optics that describes the behavior of light in nonlinear media, that is, media in which the dielectric polarization $(P)$ responds non-linearly to the electric field $E$ of the light. Hayashi and colleagues reported the batch hydrothermal synthesis of potassium niobate $\left(\mathrm{KNbO}_{3}\right)$ fine powders (synthesis time in the range 2 to $24 \mathrm{~h}$ ) using relatively low potassium hydroxide $(\mathrm{KOH})$ concentrations (in the range 0.1 to $0.5 \mathrm{M}$ that is 
much lower than the $>6 \mathrm{M}$ typically required for the CHFS of this material). Powder X-ray diffraction data of the products suggested that the phases obtained largely depended on the base concentration; at low base concentrations (0.1 M), rhombohedral phase $\mathrm{KNbO}_{3}$ poly-crystalline powders formed (similar to JCPDS reference pattern 710947) with a $\mathrm{Nb}_{2} \mathrm{O}_{5}$ impurity. At moderate base concentrations (ca. $0.25 \mathrm{M}$ ) the same major phase was formed with $\mathrm{a} \mathrm{K}_{4} \mathrm{Nb}_{6} \mathrm{O}_{17}$ impurity phase. At $0.25 \mathrm{M}$ concentration of base, the orthorhombic phase $\mathrm{KNbO}_{3}$ was formed (similar to JCPDS reference pattern 32-0822) instead of the rhombohedral phase. Importantly, when the nonlinear optical properties of the $\mathrm{KNbO}_{3}$ powders were measured and they exhibited strong intensities for second harmonic generation (SHG). To date, we are not aware of any similar reports of materials made in continuous hydrothermal flow being tested for their non-linear optical properties, which is surprising given the number of reported synthesis is flow of similar types of materials. ${ }^{30,291}$

\subsection{Nano Pigments and Thermochromics}

Nano Pigments. Inorganic pigments are used as colourants for various applications such as paints, inks and cosmetics. ${ }^{292}$ In particular, red and orange inorganic pigments are important for high value cosmetics, particularly alternatives to commercial red pigments, such as cadmium reds and oranges. More recently, perovskite-based inorganic red pigment $(\mathrm{Ca}, \mathrm{La}) \mathrm{Ta}(\mathrm{O}, \mathrm{N})_{3}$ have been proposed as $\mathrm{Cd}$-free alternatives to the commercial red pigments. ${ }^{293}$ To obtain the nitride type pigments, however, requires a high temperature, which can lead to larger particles. Other alternatives to red pigments include rubidium vanadates such as $\mathrm{Rb}_{3} \mathrm{~V}_{5} \mathrm{O}_{141}{ }^{294}$ which is considered an environmentally friendly red pigment. Other phases that might be of interest for flow reactors include the high temperature phase of bismuth vanadate, a well-known yellow pigment (that also has photocatalytic properties). ${ }^{295}$ If such pigments can made sufficiently small and with surface coatings, they will undoubtedly have applications in cosmetics or paints or inks if they can be made well dispersed to go into oil or water based formulations.

The color properties of NPs are highly complex indeed, and many NPs tend not to be ideal for high colour strength if not supported onto larger particles (which reflect the white portion of light). Also, the phenomenon of colour change with size is well known especially in systems such as gold and silver (surface plasmon resonance related). ${ }^{181}$ However, coloured NPs also have properties that make them useful in many applications. For example, inorganic particles such as titania or iron oxides in the range 50 to $300 \mathrm{~nm}$, can be used as coatings on mica to make pearl lustre pigments. ${ }^{296}$ The colour varies with coating thickness and the applications for lustre pigments include 
drinks bottles and automotive paints. CHFS synthesis can be used to reliably make titania and iron oxide in the range 5 to $50 \mathrm{~nm},{ }^{71-72,297}$ which could be ideal in such applications.

Ceramic inks from conventional pigments are usually made by firing and grinding, which requires large amount of energy and time and often materials then need to be dispersed in a further step. Batch processes have been used to make surface functionalized coloured NPs in a single step. ${ }^{298}$ Furthermore, Adschiri and co-workers have shown that continuous synthesis processes can also be used to coat larger (premade) micron size particles with dispersants, which allows them to be readily dispersed into polymer composites (or inks). ${ }^{298-299}$ Recent work by the authors has shown that complete surface coverage of citric acid on black magnetite NPs, can be achieved. ${ }^{36}$

Nano-sized gold and silver NPs have many applications including as pigments or in biological sensors ${ }^{300}$ as well as for colouring glass..$^{301}$ As seen for the section on catalysts, CHFS can be used to make nano-sized Au and Ag particles (amongst other metals), ${ }^{302}$ which could be ideal for colour applications (although lower temperature synthesis methods are also very easy to perform for these metals). ${ }^{303}$

Pure titania is brilliant white ( $>200 \mathrm{~nm}$ ) and the addition of contaminant ions to titania, can significantly change the appearance of titania. ${ }^{17}$ Titania can be doped with a wide range of ions by adding them into the respective precursor feed. ${ }^{304}$ By adding the appropriate dopants and elements into the precursor feed, mixed metal oxides can be precipitated in a fast, controllable continuous way. Titanates including potassium hexatitanate, barium titanite and barium strontium titanate, have been successfully manufactured via CHFS, as well as zirconates such as barium zirconate and ceria zirconate. ${ }^{75,30579,306}$

Doping of other known ceramic pigments via CHFS, could lead to new colours or new methods of making known pigments faster. Research in the field of ceramic pigments is particularly orientated towards enlargement of the chromatic set of colours. ${ }^{307}$ Coloured oxides, which are known for pigment properties include iron oxides, for which several examples are known, e.g. iron oxide NPs were made by Sue and Co-workers who made them using collision type micromixers using iron nitrate precursors. ${ }^{308} \alpha-\mathrm{Fe}_{2} \mathrm{O}_{3}$ (hematite) can also be made using relatively simple reagents in CHFS using ferric nitrate. ${ }^{309}$ In the future, CHFS syntheses of pigments will have several possible interesting possibilities; a flow system offers good consistency in the preparation and production of colours, with little or no batch to batch variations. This could also allow industry to "dial in" the exact colours it needs upon demand, rather than relying on the mixing of colour palates of different powders (a high skilled and complex job). Many of these pigments can be made exceedingly finely or with organic coatings to compatibilise them with creams or formula- 
tions for cosmetics. Alternatively, NPs can be formulated into with inkjet printable inks or mixed with polymers for composites. Indeed, the use of multifunctional pigments as "all in one" pigments and UV attenuators, may be achievable in the future.

Thermochromic Nanoparticles. Chromeogenic solid state materials exhibit changes in their optical properties due to some external stimulus; most common of these are photochromic and thermochromic materials, where the stimuli are light irradiation (photons) and change in temperature, respectively. ${ }^{310}$ Thus, thermochromic materials change colour at a characteristic 'transition temperature'. The thermochromic effect occurs over a range of temperatures, which makes them invaluable in a wide range of solar control applications such as energy saving windows..$^{310}$

Due to its thermochromic properties, monoclinic (doped or undoped) vanadium dioxide, $\mathrm{VO}_{2}$, is a material for energy-efficient, intelligent window applications and building materials. ${ }^{310}$ This involves a key thermal phase transition from a monoclinic structure into a rutile phase $\left(\mathrm{VO}_{2}-\mathrm{R}\right)$, with each pair of vanadium ions forming a $\mathrm{V}-\mathrm{V}$ bond; when electrons in the $\mathrm{V}-\mathrm{V}$ bond are released; this results in a change in band structure. ${ }^{310}$ The transition causes a change from semiconducting to semi-metallic behavior, which results in a change in electrical conductivity and in the near-IR transmittance. Consequently, the $\mathrm{VO}_{2}$ coating is highly transmissive of long-wave thermal radiation at ambient conditions, but it becomes reflective above the metal-to-semiconductor transition (MST) temperature, hence, its application for thermochromic materials (see Figure 10). ${ }^{310}$

Figure 10.

To date, CHFS processes have been used to develop monoclinic $\mathrm{VO}_{2}$ thermochromics, for example a two-step synthesis route used CHFS to make $\mathrm{VO}_{2}$ followed by a short post heat-treatment step. ${ }^{311}$ This is a significant achievement because the monoclinic $\mathrm{VO}_{2}$ phase is normally made over several hours in batch a temperature in excess of $200^{\circ} \mathrm{C}$. The authors are very confident that the high temperature phase will one day be accessible if a longer residence time on the order of a minute or less (in excess of $370^{\circ} \mathrm{C}$ or so for the reaction temperature), can be used in flow to only give the monoclinic phase. When monoclinic $\mathrm{VO}_{2}$ thermochromics were made via the CHFS route (plus a short heat-treatment), a range of particle sizes were obtained in the first step that were dependent on CHFS synthesis conditions (size range 50 to $200 \mathrm{~nm}$ by varying reaction temperatures and the residence times in the 
process). ${ }^{311}$ The NPs were crystalline with rod and sphere-like morphologies present, with the size of both the rod and spherical particles being dependent on reaction temperature and residence time. SEM micrographs showed the surface of the CHFS powders was uniform. After a short post-synthesis heat-treatment, the materials were completely phase-pure monoclinic $\mathrm{VO}_{2}$ and this led to them exhibiting a large and reversible switch in optical properties (at near-IR wavelengths). Thus, if such materials can be incorporated into coatings or in composites, they could be used for fenestration in architectural applications..$^{311}$

Unfortunately, the MST temperature of pure $\mathrm{VO}_{2}\left(\mathrm{ca} .68^{\circ} \mathrm{C}\right)$ is too high for practical applications and different approaches have been followed to decrease this temperature to $25^{\circ} \mathrm{C}$. In one $\mathrm{CHFS}$ synthesis study, Nb-doping was used to lower the MST phase transition temperature, possibly due to inducement of strain in the $\mathrm{VO}_{2}$ structure, favoring the transition into a tetragonal (rutile) arrangement. $9^{\circ}$ The doped materials showed a decrease in the MST temperature and a slight improvement in the reflection of long-wavelength radiation at high temperature (for optimum Nb-loading). The materials showed a decrease in the MST transition temperature of $c a .20^{\circ} \mathrm{C}$ for samples containing relatively high $\mathrm{Nb}$ levels ( $>5$ at\%). ${ }^{90}$ However, these samples revealed a comparable change in near-IR transmittance compared to undoped $\mathrm{VO}_{2}$ made under similar conditions. The incorporation of low Nb concentrations $(<1$ at\%) gave improved transmittance modulation, which was likely to be due to the formation of plate-like $\mathrm{NPs}$, which have been widely observed in effective $\mathrm{Nb}$-containing $\mathrm{VO}_{2}$ materials. ${ }^{\circ 0}$

In the future, there are many other materials that have not yet been made via CHFS methods and tested, which are known as solid state thermochromics. These materials include $\mathrm{V}_{2} \mathrm{O}_{31}, \mathrm{~V}_{2} \mathrm{O}_{5}, \mathrm{~V}_{6} \mathrm{O}_{13}$ and $\mathrm{Ti}_{n} \mathrm{O}_{2 n+1}$ magnéli phases..$^{310}$ Other thermochromics include lanthanide based compounds such as a $\mathrm{RNiO}_{3}$, where $\mathrm{R}$ is a rare earth element; all of these exhibit a metal-insulator transition temperature of $130 \mathrm{~K}\left(-140^{\circ} \mathrm{C}\right), 200 \mathrm{~K}\left(-70^{\circ} \mathrm{C}\right), 400 \mathrm{~K}\left(130^{\circ} \mathrm{C}\right)$ and $560 \mathrm{~K}$ $\left(290^{\circ} \mathrm{C}\right)$ for $\mathrm{R}=\mathrm{Pr}, \mathrm{Nd}, \mathrm{Sm}$ and $\mathrm{Gd}$, respectively..$^{310}$

\section{NANOCERAMICS FOR HEALTHCARE}

Whilst there are still fears about human exposure to nanoparticles in the environment, 35 conversely, smart nanoparticles are increasingly being considered for healthcare. ${ }^{182}$ This is because NPs can be functionalized ${ }^{312}$ for a host of uses and as they are far smaller than cells (typically tens of microns) they can be often be delivered where required (such as via injection). Therapeutic and diagnostic nanoparticles generally come into two categories that are (i) inorganic nanoparticles (e.g., metals such as $\mathrm{Au}, \mathrm{Ag}, \mathrm{Pt}, \mathrm{Pd}$, and semiconductors such as $\mathrm{CdSe}, \mathrm{CdS}, \mathrm{TiO}_{2}$, $\mathrm{PbS}$, Si and 
magnetic compounds such as $\mathrm{Fe}_{3} \mathrm{O}_{4}, \mathrm{Co}, \mathrm{CoFe}_{2} \mathrm{O}_{4}$, FePt, CoPt, etc.) and (ii) organic nano or microparticles (e.g., micelles, liposomes, etc. $)^{182}$

Nanoceramics for healthcare can cover a wide range of categories such as antimicrobials, ${ }^{313}$ bioceramics $^{314}$ (to replace hard tissue or in biomedical composites) as well as advanced NPs for therapies or to assist in diagnosis or information gathering (drug delivery, ${ }^{315}$ imaging, sensing and therapeutic intervention ${ }^{117},{ }^{182,}{ }^{316}$ and for the understanding of basic biological processes). For the latter, NPs can be engineered with distinctive size, shape, composition, and surface functionalisation, to facilitate a wide range of therapeutic or biological applications. ${ }^{182,315}$ For example, the unique properties of NPs made via CHFS processes, may enable better study of fundamental biological processes.

We will now discuss areas where continuous hydrothermal synthesis of nanomaterials has been used in healthcare as well as a few areas, which could benefit from being made via this technology in the future. The review called "Nanoparticles in the clinic" by Aaron C. Anselmo and Samir Mitragotri, is recommended as a general introduction to those seeking more information on the general applications of nanoparticles in therapeutics and diagnostics. ${ }^{182}$ Furthermore, the excellent review By Adschiri and Byrappa et al. that covers the use of Supercritical Fluids for making NPs that have biomedical applications. ${ }^{117}$

Surface Biomedical Coatings and Safety of Therapeutic NPs. Surface functionalisation of NPs for healthcare might be carried out for a number of reasons. Functionalisation may be required to reduce agglomeration, to make them more biocompatible, to bind a drug (which may become released), or to bind a fluorescent tag or to adding receptor groups to the particle surface that might be then able to bind to target antibodies or specific cells or other things in the body (for targeted delivery). ${ }^{117,217,312,316}$ Achieving certain types of surface functionality is often difficult for some biological NPs. ${ }^{312}$ Thus, EDC coupling (EDC = 1-ethyl-3-(dimethylaminopropyl) carbodiimide hydrochloride) can be used to covalently link carboxylic acids on the particle surface to amines via formation of a "zero length" amide bond (assuming the carboxylic acid can be pendant from the particle surface, e.g. from a citrate molecule). ${ }^{312}$ The key advantage of this procedure is that it involves no lengthy prep..$^{312}$

It is notable that when NPs are considered for use in therapeutics, they are generally done so after ensuring that they are safe to use.317 Despite the numbers of relevant nanoparticles that have been made via continuous hydrothermal flow methods, the cost and effort in fulfilling necessary medical regulations, represents a significant barrier 
to such materials actually being used in therapeutic medical applications in humans. It is noted that biomedical and other materials often have very high standards in terms of trace heavy or other metal contents, ${ }^{318}$ which may represent a challenge for hydrothermal flow systems to reach. Hereafter, we will discuss a range of relevant materials and applications in healthcare.

\subsection{Magnetic Particles for Healthcare}

Globally, 14 million new cases of cancer are diagnosed annually with 8.2 million cancer-related deaths. ${ }^{39-320}$ Cancer diagnoses are projected to increase ca. 70\% worldwide in the next two decades, according to the WHO (World Cancer Report 2014). Cancer is the second leading cause of death in non-communicable diseases and this has a disproportionate effect on the less well off. ${ }^{319-320}$ The use of NPs in healthcare may be able to offer support in the battle to treat cancer or for supporting clinicians to reduce side effects or minimise discomfort to cancer patients. Some of the most promising work in this area has been in the use of magnetic NPs.

Magnetic Hyperthermia. Magnetic hyperthermia using surface functionalised NPs, is an therapy that attempts to controllably heat and cause the selective death of cancer cells from the inside (see figure 11). ${ }^{221}$ Early clinical studies have already been conducted with positive results..$^{322}$ In this therapy, magnetic NPs are injected into a tumour site so that they can be absorbed by cancer cells (which tend to be more porous) and an AC magnetic field is applied for a fixed time that causes the particles to heat up. The heating occurs because below a critical size, $\mathrm{Fe}_{3} \mathrm{O}_{4}$ behaves as a superparamagnetic single domain crystal, i.e. a single giant magnetic moment, sum of all the individual magnetic moments carried by the atoms in the NP. The magnetic moments of the particles are initially randomly oriented (due to thermal fluctuations with no applied field). However, when the AC magnetic field is applied, the magnetic moments of the particles can flip rapidly, which causes heating via primary and secondary mechanisms;

(i) Primary heating mechanism: The dissipated magnetic energy is converted to thermal energy (heat) known as Neel relaxation (from rotation of the magnetic moment from within the particle).

(ii) Secondary heating mechanism: particles can physically rotate as they try to align themselves to the applied field (due to changes in the magnetic moments), which induces frictional heating. 
Due to their greater porosity, cancer cells tend to take up larger amounts of MNPs than normal cells, which is helpful during magnetic hyperthermia treatments of cancer. Different mechanisms of cell damage can be initiated depending on clinical needs. These are "hyperthermia" and "thermoablation"; in the former, the treatment is controlled to limit temperatures to the range 42 to $45^{\circ} \mathrm{C}$ for up to few hours. This may be in combination with other therapies (such as chemotherapy or indeed irradiation). In thermoablation, temperatures $>50{ }^{\circ} \mathrm{C}$ are used in the tumour area (exposure times of a few minutes to kill tumour cells. ${ }^{321}$ Care must be taken to avoid overheating and shock syndrome due to sudden release of large amounts of necrotic tumour material and triggering a major inflammatory response.

Specifically, magnetic nanomaterials in the body require special surface coatings, e.g. using dextran or other biocompatible materials ${ }^{312}$ and also regulatory approval before being administered into humans, which inevitably increases costs. Although there is no reported use of CHFS processes to produce such materials for testing in the body yet, magnetite synthesis has been made with surface coatings via a single step CHFS process, thus, this synthesis route could offer tremendous advantages as it is able to reproducibly make magnetic NPs with well-defined properties. ${ }^{36,323-327}$

A recent approach to make more tuneable magnetic hyperthermia systems (to better control heating rates) involved the use multiphase composite materials, e.g. $\mathrm{SrFe}_{12} \mathrm{O}_{19} / \mathrm{MgFe}_{2} \mathrm{O}_{4} / \mathrm{ZrO}_{2}$ composites, where the net contributions of different magnetic phases, made it possible to modify the magnetization and anisotropy of the composite material. ${ }^{328}$ To date, such materials have been made using more conventional synthesis approaches such as citratebased chemistry. ${ }^{328}$ Clearly, such composites could be made efficiently in CHFS reactors; if this was also coupled to biocompatible coatings, it would be an exciting approach to the development of completely new materials for hyperthermia. In the opinion of the authors, this is clearly an area for future development.

There have been several reports on the CHFS synthesis of (water) dispersible magnetites..$^{323-326}$ This includes the use a "pulsed" flow system that provided excellent heating and cooling rates, with the ability of using the same reactor both for NP production and in situ synchrotron studies. ${ }^{329}$ The researchers observed that by using iron citrate as a precursor, they could obtain particles of ca $20 \mathrm{~nm}$ or so. ${ }^{329}$ Some of the UCL authors also demonstrated the CHFS synthesis of citric acid coated ferro-fluids; surface functionalization was achieved through introducing a supplementary flow of capping agent (citric acid) to the stream of nascent (newly formed) NPs. ${ }^{36}$ It was found that certain process conditions were critical to effective functionalization of the nascent NP stream, and that high grafting densities 
of the capping agent were obtained in a relatively narrow process window. A method for scaling-up the reactor was also described using a pilot plant at ca. $1 \mathrm{~kg} \cdot \mathrm{h}^{-1}$ (see later in section 10.7). $\cdot^{6}$

In 2012 Adschiri and co-workers, reported the synthesis of water dispersible magnetite $\left(\mathrm{Fe}_{3} \mathrm{O}_{4}\right) \mathrm{NPs}$ coated with 3,4-dihydroxyhydrocinnamic acid (DHCA) using a tubular flow reactor. ${ }^{327}$ As in the case for citric acid coated magnetite, ${ }^{36}$ the $\mathrm{Fe}_{3} \mathrm{O}_{4} \mathrm{NPs}$ were well dispersed in water because the surfaces of the NPs were fully covered by DHCA molecules and the $-\mathrm{COOH}$ groups in the DHCA molecules were exposed to the surrounding water. ${ }^{327}$ In cell studies involving the magnetic NPs, cytokines such as IL-12 and TNF-alpha, were not produced from the dendritic cells of mice, which indicated no substantial immune response was observed for these cells. ${ }^{327}$ Therefore, it was suggested that the $\mathrm{Fe}_{3} \mathrm{O}_{4} \mathrm{NPs}$ might be suitable for biological applications such as magnetic resonance imaging contrast agents and carriers for drug and gene delivery, and potentially for hyperthermia therapy. ${ }^{327}$

Other superparamagnetic NPs made via continuous hydrothermal processes include the ca. $20 \mathrm{~nm}$ size superparamagnetic $\mathrm{MgFe}_{2} \mathrm{O}_{4} \mathrm{NPs}$ that were also made in a flow-type apparatus at $460^{\circ} \mathrm{C}$ (30 MPa) using the $\mathrm{Mg}$ :Fe ratios of $1: 1$ and 1.5:1.330 High reaction temperature and non-stoichiometric reaction conditions were deemed to be key to getting the phase pure product. This non-stoichiometry was similar to that needed in a report for synthesis of barium hexaferrite using a flow-type apparatus used by Hakuta et al. ${ }^{77}$

Figure 11

Magnetic Resonance Imaging. Other noteworthy biomedical materials that are of interest for magnetic resonance imaging, have been made via CHFS in the work of Tang ${ }^{213}$ for the synthesis of $\mathrm{Fe}_{2} \mathrm{O}_{31}, \mathrm{Fe}_{3} \mathrm{O}_{41} \mathrm{Gd}(\mathrm{OH})_{3}$ and $\mathrm{Co}_{3} \mathrm{O}_{4 i}$ their capacity as contrast agents for magnetic resonance imaging was studied. Relaxivity data for the $\mathrm{Fe}_{3} \mathrm{O}_{4}$ based materials, showed values comparable to some commercial contrast agents. ${ }^{213}$

Magnetic Susceptometry (Locating Lymph Nodes). Magnetic nanoparticles can be used to find the location of lymph nodes for biopsies in screening for breast cancer. A spinout of University College London called Endomagnetics $^{319}$ has developed a portable instrument called Sentimag ${ }^{\circledR}$ that uses the principle of magnetic susceptometry to help locate lymph nodes under the arms. If a breast cancer patient requires a biopsy to search for any spreading of 
the primary cancer, biopsies of the lymph nodes, can be taken from under the arms. However, without guidance, this can be a rather difficult process.

Firstly, to reveal the precise location of the lymph nodes, the breast of the patient is injected with medically approved magnetic NPs that are then aided to accumulate into the lymph nodes under the arms. When the surgeon then makes an incision under the arms, the Sentimag ${ }^{\circledR}$ device generates an alternating magnetic field, which transiently magnetises the medical grade iron oxide particles in the lymph nodes. The tiny magnetic signature generated by the particles is then detected by a Sentimag ${ }^{\circledR}$ probe (that looks like a small wand). As the wand gets closer to the NPs in the lymph nodes, a change in the pitch of the machine, informs the clinician of the best and precise locations

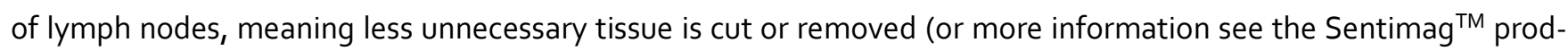
uct). ${ }^{319}$

In closing, there is much scope in the future to use surface functionalised magnetic NPs in therapeutic treatments as well as for aiding surgeons to locate soft tissues such as for biopsies or for MRI or other imaging techniques. The advantage of making such particles in flow processes is that such particles can easily be doped or tuned for size (which affects final properties). The flow process also allows surface coating to be applied, which will aid biocompatibility of the NPs.

\subsection{Antimicrobials}

The presence of dangerous hospital-acquired infections (HAls) including surgical site infections is ca. $300,000 \mathrm{NHS}$ patients hospital in England every year. ${ }^{33^{1}}$ Rigorous hospital cleaning regimes have helped to reduce hospital contamination on surfaces, however, it is difficult to completely eliminate surface contamination or keep low bacterial loads. One strategy to reduce the cycle of bacteria transmission in hospitals is to utilize self-sterilizing surfaces. ${ }^{33^{1}}$ This includes the use of microbiocide-releasing surfaces including those based on copper or silver or photobactericidal approaches. In the latter, light-activated mechanisms in which a host of reactive oxygen species (ros) are generated, are of particular interest, since the non-site specific mode of bacterial attack would be less likely to be countered by bacterial resistance.

Continuous hydrothermal processes have been used in the manufacture of fine particle silver and copper based compounds, although they have not been measured for antibacterial properties, these materials should be effective in such applications. Metallic particles are readily accessible via hydrothermal processes. For example, supercritical 
water $^{152}$ or sc-methanol70 flow systems were used to reduce the precursors to the base metals. Hayashi and coworkers also reported the synthesis of PVP-coated copper NPs, which are stable to oxidation in alcoholic dispersions (> 1 year stability in ethanol as evaluated by shifts in the surface plasmon resonance band of product solutions). The precursors copper formate and polyvinylpyrrolidone (PVP) in a aqueous solution were mixed with a heated feed of formic acid (aq.) before passing into a heating zone (reaction was at $400{ }^{\circ} \mathrm{C}, 30 \mathrm{MPa}, 1.1 \mathrm{~s}$ mean residence time). ${ }^{131^{1}}$ 332 The reaction pathway was suggested to be the step-wise hydrolysis of copper formate, followed by dehydration to oxide products and subsequent reduction by in situ made hydrogen (derived from precursor and formic acid decomposition). The Cu NPs were ca. $18 \mathrm{~nm}$ in size and spherical in shape and contained no oxide contaminants. Conductive films (0.33 microns in thickness) were prepared from the Cu NP conductive inks. Lu Zhou and co-workers reported that the particle size of Cu NPs could be controlled and that the size was very sensitive to the $\mathrm{pH}$ and concentration of precursor (copper sulfate). 333 When the concentration of copper salt increased from 0.05 to $0.5 \mathrm{~mol}^{-\mathrm{L}^{-1}}$, the average particle size increased from 14 to $50 \mathrm{~nm}$. Furthermore, when the molar ratio of the $\mathrm{NaOH}$ (base) to copper salt increased from o:1 to 2:1, the average particle size went from 85 to $14 \mathrm{~nm}$. The concentration of complexing agent ethylenediamine tetra-acetic acid (EDTA) was shown to improve the homogeneity and dispersity of the Cu particles. 333 Supercritical methanol has also been used to make Cu NPs in flow [as well as Ag particles and Ni (metal) / Ni hydroxide mixtures].70

The authors have also developed small nano-CuO particles (which are converted to reduced copper(I) oxide species or copper metal particles under use) for the reduction of $\mathrm{CO}_{2}$ via heterogeneous catalysis 334 or electrocatalytic reduction 335 (in a Nafion ink), the details of which can be found elsewhere in section 7.2.1. (non-precious metal catalysts / supports).

Continuous hydrothermal synthesis was used on the pilot plant scale for the preparation of $\mathrm{TiO}_{2}$ particles, which were embedded into a matrix to give UV-activated photobactericidal polymers. ${ }^{313} \mathrm{Au} / \mathrm{TiO}_{2} \mathrm{NPs}$ were also used by coating the nano-titania with gold particle using a simple reflux process. Both sets of particles were also surfacefunctionalized (heated the powders in excess oleic acid with a catalytic amount of triethylamine to encourage ester formation between the titanol groups on the particle surfaces and the oleic acid) before being added into the polymer. The powders were mixed into commercially available medical grade silicone, using a simple dipping technique and the antimicrobial activity of the NP incorporated polymers, was tested against both Gram-negative and Grampositive bacteria, commonly found in healthcare environments. ${ }^{313}$ A UVA source ( $365 \mathrm{~nm}$ ) was used to stimulate the 
photobactericidal properties of these composite polymers (Figure 12). The antibacterial composites were tested against $\mathrm{S}$. aureus and it was found that unprecedented kills were achieved with bacterial numbers reduced to below detection limits (<15 minutes), without the need for any UV treatment (dark conditions). Interestingly, a small 'dark kill' effect was noted when tested against E.coli, after exposure to UV illumination ( $365 \mathrm{~nm}$ ) for 95 minutes, whereby bacterial numbers were reduced to below the detection limit. ${ }^{313}$ These surfaces induced the lethal photosensitisation of E. coli within 95 minutes, with bacterial numbers reduced to below the detection limit. ${ }^{133}$ They also exhibited efficacious antimicrobial activity against S. aureus via a non-light-activated mechanism, reducing bacterial numbers to below the detection limit within 15 minutes. The incorporation of NPs did not affect polymer colouration, a highly desirable property from a commercial viewpoint.

Figure 12

Recently, Kellici et al. ${ }^{336}$ reported the preparation of reduced graphene oxides ( $\mathrm{rGOs}$ ) with antibacterial properties. The materials were prepared from an aqueous solution of $\mathrm{GO}$ that was pumped to mix with a flow of $\mathrm{KOH}$ solution at room temperature at a T-junction. This mixture was then brought into contact with supercritical water in counter-current (mixer) reactor, whereupon reduction of graphene oxide (to rGO) occurred. The antibacterial properties of GO and rGO were tested against gram negative bacterium Escherichia coli (E. coli) to determine the minimum inhibitory concentration (MIC). The MIC for ( $\mathrm{rGO}$ ) was the same as that for reduced graphene oxide produced via a more conventional reduction method. ${ }^{336}$ In related report, Kellici and colleagues, made Ag graphene nanocomposites [with p-hexasulfonic acid calix[6]arene (SCX6) as a particle stabilizer], with good antibacterial properties and low cytotoxicity. ${ }^{337}$ The nanocomposites showed excellent activity against E. coli (Gram-negative bacteria) and S. aureus (Gram-positive) bacteria. 337

\subsection{Bioceramics (Calcium Phosphates)}

Biomaterials are a class of synthetic materials, which are designed to replace or fix natural hard and soft tissues in the body that have been lost due to disease or accident or other causes. Such materials include prosthetics, implants, bone grafts, dental cements,338-342 bone cements, $338-343$ dentures, plates, screws, organs, skin grafts, etc. 
Bone is a specialized tissue comprised of mineral substances, organic tissues and water. ${ }^{344-345}$ The inorganic nano-apatite rods in bone (which are $<100 \mathrm{~nm}$ ) are arranged in lamellae and bound to collagen (Figure 13a) in a hierarchical structure. The mineral or inorganic component is also known as biological apatite and comprises calcium, phosphate and hydroxyl ions with small amounts fluoride, carbonate, magnesium, and potassium as well as other ions. These ions can play an important role in the chemical and biological behavior of biological apatite. ${ }^{344-345}$

Synthetic bone substitutes (such as bioceramics bone grafts) have been developed to restore bone that has been lost since there is often insufficient availability of autografts (bone harvested from the patient). ${ }^{346}$ Bioceramics are considered to be in one of several classes; bioinert (e.g. alumina, zirconia), resorbable (e.g. tri-calcium phosphate) and bioactive (e.g. bioactive glasses ${ }^{345}$ and hydroxyapatite), which can also be porous for tissue ingrowth. 345

The lack of availability high quality biomedical ceramics at an affordable cost in developing countries, is often overlooked and in cases where such implants are not available in emergencies (e.g. earthquakes in remote areas), it often requires amputation of broken limbs (to save life), rather than rehabilitation of the defect site with an implant. For this reason, the lead author and others have supported the development of low cost biomedical ceramics in the developing nation of Pakistan. ${ }^{347}$ Many of the ideas of continuous or batch or automated synthesis in plastic vessels rather than metal pipes or reactors (albeit at relatively mild conditions compared to CHFS), are being evaluated and attempted for scale-up in the future $347-350$

Figure 13

Perhaps the most well know bioactive bioceramic is synthetic hydroxyapatite $\left[\mathrm{HA}, \mathrm{Ca}_{10}\left(\mathrm{PO}_{4}\right)_{6}(\mathrm{OH})_{2}\right]$, which is chemically similar to biological apatite. ${ }^{346}$ Thus, synthetic HA is of interest as a biocompatible phase / reinforcement in biomedical composites, ${ }^{351}$ for potentially filling bulk bone defects and for coatings on metal implants. HA and other calcium phosphates (e.g. calcium deficient hydroxyapatite, $\mathrm{CDHA}$ ) are also of interest as components in injectable bone cements.

HA powders can be routinely synthesized ${ }^{314}$ via techniques such as co-precipitation, mechanochemical methods, sol-gel processing, emulsion methods, batch hydrothermal processes and chemical vapour deposition. ${ }^{352}$ The disadvantages of these methods include, they are time consuming, often require very precise control over reaction conditions, require expensive starting materials or large amounts of toxic organic solvents and little or no control 
over HA particle characteristics can be achieved (i.e. particle size, agglomeration surface area, shape, etc.). ${ }^{314}$ For example, when conducting wet chemical batch synthesis of HA at room temperature, a long maturation step (often days) followed by a heat-treatment $>600^{\circ} \mathrm{C}$, is often required. 353 If sufficient maturation time is not allowed, a nonstoichiometric (Ca:P ratio not 1.67) phase separated product is obtained upon heat-treatment (which can affect biological properties in vivo). Batch reactions can include organic templating agents, ${ }^{354}$ which are surface bound and therefore potentially unsuitable for biomedical applications.

The synthesis of nano-size calcium phosphate bioceramics via CHFS was first reported in a communication entitled "instant hydroxyapatite" by one of the authors..$^{76}$ In the report, it was shown that not only was the crystallinity of HA controllable via the process temperature, but also that the Ca:P ratio could be controlled by changing the temperature or $\mathrm{pH}$ of the reaction. In the report, basic solutions of calcium nitrate and ammonium phosphate, respectively, were pumped to meet at a T-piece mixer, with supercritical water feed at up to $400^{\circ} \mathrm{C}$ and at $24 \mathrm{MPa}$ (Figure 13b). This initial mixture was then brought to meet a superheated water feed in a reactive mixer, whereupon, a rapid reaction occurred. ${ }^{76}$ The dried product was obtained as a highly crystalline and phase pure material, without the need for an ageing step or subsequent heat-treatment (this can be compared to the several days or hours maturation step mentioned earlier for standard co-precipitation/water based sol-gel reactions353).

Transmission electron microscope (TEM) images of the HA powders synthesized with superheated water in flow at $200^{\circ} \mathrm{C}$ [denoted as sample $\mathrm{HA}(200)$, where the number in brackets is equal to the incoming superheated water feed temperature], revealed small agglomerates. TEM images for $\mathrm{HA}(300)$, showed well defined rods of ca. $15 \times 65$ $\mathrm{nm}$ (aspect ratio ca. 4.3). The TEM image for $\mathrm{HA}(400)$, (figure $13 \mathrm{~b}$ ) revealed well defined crystalline rods of ca. $140 \mathrm{x}$ $40 \mathrm{~nm}$ (average aspect ratio ca. 3.5). Thus, the size of rods obtained was thus, similar in size to the biological nanoapatites found in bone itself (Figure 13a). ${ }^{76}$

BET surface area measurements for CHFS made HAs and calcium deficient apatite (CDHA), were measured for samples that were made using a superheated water feed in the temperature range 300 to $400{ }^{\circ} \mathrm{C}$; powders were found to possess BET surface areas in the range 39 to $100 \mathrm{~m}^{2} \mathrm{~g}^{-1}$. The crystallinity of the nano-powders was assessed by both X-ray powder diffraction (XRD) and Fourier transform infrared (FTIR) spectroscopy. The XRD patterns for samples $\mathrm{HA}(300), \mathrm{CDHA}(400)$ and $\mathrm{HA}(400)$, respectively, showed a good match to phase pure $\mathrm{HA}$ [similar to similar to JCPDS reference pattern o9-0432 (I)-Hydroxyapatite, syn- $\mathrm{Ca}_{5}\left(\mathrm{PO}_{4}\right)_{3}(\mathrm{OH})$ ]. The XRD peaks became increasingly 
sharper and well resolved, with increasing synthesis temperature, the $\mathrm{CDHA}(400)$ sample XRD peaks being noticeably less sharp than the $\mathrm{HA}(400)$ sample peaks.

Since the early reports of calcium phosphates being made via CHFS, Tang was able to use continuous hydrothermal processes to make different morphologies of hydroxyapatite. Attempts to introduce a drug and cations to the structure / surface of hydroxyapatite was also conducted and cell proliferation and bone nodule assays were used to evaluate the hydroxyapatite synthesized via this route. This work revealed that no significant cytotoxic effect on mouse embryonic stem cells occurred. ${ }^{213}$

As well as simple calcium phosphates being used as bioceramics, there is also a need to more closely mimic the chemical and structural component in mineral bone (i.e. in biological apatite). Human bone mineral typically contains carbonate ions ( 8 wt\%) that occupy the normal phosphate and hydroxide sites. It has been shown that carbonate presence in synthetic HA, leads to structural disorder and a higher solubility. Therefore, carbonated hydroxyapatite ( $\mathrm{CHA})$, is considered as an optimized biomaterial, which leads to faster bonding between an inserted body and human bone. Substitution of carbonate ions in the HA lattice can be of two types, A-type, where carbonate ion substitutes the hydroxyl ion and B-type, where the carbonate ion substitutes the phosphate ion. ${ }^{125}$ Atype carbonated hydroxyapatite (CHA), is normally formed when carbon dioxide gas is flow over $\mathrm{HA}$ at a high temperature. B-type $\mathrm{CHA}$ is formed by precipitation. In a modification to the synthesis of "instant $\mathrm{HA}^{\prime}{ }^{76}$ carbonate substituted hydroxyapatite (CHA) was prepared using CHFS. ${ }^{76,124}$ The carbonate content obtained was $\approx 5$ wt\%, which was comparable or more than some of the previously reported values in the literature. ${ }^{125}$ Urea was used as a carbonate source in some reactions and it was found that increasing the urea concentration in solution above a threshold, resulted in biphasic mixtures of carbonate-substituted hydroxyapatite and calcium carbonate.

The rapid continuous hydrothermal synthesis of carbonated hydroxyapatites (CHAs) was also investigated by $\mathrm{Yu}$ et al. in a tubular reactor. ${ }^{130}$ The effects of reaction time and the ratio of $\mathrm{PO}_{4}{ }^{3-} / \mathrm{CO}_{3}{ }^{2-}$ on the apatite structure, were investigated. The main results seem to suggest that $30 \mathrm{~s}$ residence time was enough to produce fully crystalline $\mathrm{CHA}$ nano-rods, ca. $20 \times 70 \mathrm{~nm}$ in size (at $24 \mathrm{MPa}$ ). ${ }^{130}$ However, from the authors own experience, ${ }^{76,124}$ we would suggest it should actually take considerably less time to be formed under these conditions, in fact a few seconds should suffice.

Following pioneering research of the highly distinguished scientist and children's book writer Professor Larry Hench 355 in the use of silicon as a biogenic element in bioglass formulations, ${ }^{345}$ it is known that such biomaterials can 
release silicon into surrounding tissues after implantation, which can accelerate bone growth. 345 Thus, silicon substituted HA is of interest in this regard as a bioactive ceramic. ${ }^{125}$ The CHFS synthesis of silicate-substituted hydroxyapatite $\left(\mathrm{SiO}_{4}-\mathrm{HA}\right)$ was conducted using silicon acetate as a silicate ion source. ${ }^{124}$ It was observed that a substitution threshold of $\sim 1.1 \mathrm{wt} \%$ existed for synthesis of $\mathrm{SiO}_{4}-\mathrm{HA}$ in the CHFS system, which could be due to the decreasing yields with progressive increase in silicon acetate concentration. ${ }^{124}$

As well as carbonate and silicate ions, metal ions can be substituted into synthetic apatites and play an important role in their chemical and biological behaviors. In $\mathrm{Mg}^{2+}$ containing biological apatites such as those found in pathological calcifications, human dental calculus or carious lesions, a magnesium stabilized $\beta$-TCP (Mg- $\beta$ TCP) or Whitlockite $\left[\mathrm{Ca}_{18} \mathrm{Mg}_{2} \mathrm{H}_{2}\left(\mathrm{PO}_{4}\right)_{14}\right]$ is often formed. Dentine, enamel and bone contain up to $1.1,0.4$ and $1.0 \mathrm{wt} \% \mathrm{Mg}^{2+}$ ions, respectively. In calcified tissues, the $\mathrm{Mg}^{2+}$ content is higher at the beginning of the calcification process and decreases with increasing calcification in newborn babies. Mg depletion in aged people, results in decreased osteoblastic and osteoclastic activities and bone fragility. $\mathrm{Mg}^{2+}$ substitution for $\mathrm{Ca}^{2+}$ in $\mathrm{HA}$, reduces crystallinity, increases solubility, and lowers the temperature for conversion of HA into $\beta$-TCP. However, an excess of $\mathrm{Mg}^{2+}$ is known to reduce bioactivity in such materials.

In a report by the authors, CHFS was used to directly produce a range of magnesium doped calcium phosphates.356 Initially, magnesium substituted hydroxyapatite, $\mathrm{Mg}-\mathrm{HA}\left[\mathrm{Ca}_{10-x} \mathrm{Mg}_{\mathrm{x}}\left(\mathrm{PO}_{4}\right)_{6}(\mathrm{OH})_{2}\right]$ was prepared for $\mathrm{x}$ $=0.2$. Biphasic mixtures of $\mathrm{Mg}-\mathrm{HA}$ and Whitlockite were obtained for the range $\mathrm{x}=0.4$ to 0.8 . In the report, the first CHFS synthesis of crystalline Whitlockite $\left[\left(\mathrm{Ca}_{3-y} \mathrm{Mg}_{y}\left(\mathrm{HPO}_{4}\right)_{2}\left(\mathrm{PO}_{4}\right)_{2-2 z / 3}\right]\right.$, was reported using reagents in the range $\mathrm{x}=$ 1.6 to $5.3 .3^{356}$ With increasing $\mathrm{Mg}^{2+}$ substitution (for $\mathrm{Ca}^{2+}$ ), the material became ever more amorphous and surface area was observed to increase. Transmission electron microscopy revealed that in the case of $\mathrm{x}=3.9 \mathrm{where}, \mathrm{Mg}$ substituted TCP material was made in the CHFS process, the particles were hollow spheres of ca. $28 \mathrm{~nm}$ in diameter. 356

In order to develop high strength bioceramics and other ceramics, there has been interest in consolidation methods, particularly ones in which the nano-grained structure can be retained and high density can be achieved. For example, the synthesis of high-strength, completely dense nanograined hydroxyapatite (bioceramic) monoliths are considered a challenge as high temperatures or long sintering times are often required. Some of the UCL authors investigated the use of spark plasma sintering of CHFS made nano-rods of hydroxyapatite (HA) and calciumdeficient $\mathrm{HA}$, to full theoretical density in $\sim 5 \mathrm{~min}$ at temperatures up to $1000{ }^{\circ} \mathrm{C} .{ }^{127}$ After significant optimization, 
fully dense HA discs were obtained, which were translucent (almost transparent!), suggesting they were fully dense (see Figure 14). Significantly high three-point flexural strength values for such materials (up to $158 \mathrm{MPa}$ ) were measured, which are amongst the highest values reported in the literature. ${ }^{127}$ Freeze-fracturing of disks followed by scanning electron microscopy investigations, revealed selected samples possessed sub-200 nm sized grains and no visible pores, confirming they were fully densified. ${ }^{127}$

Figure 14.

In the follow-up report, the authors prepared nano-sized hydroxyapatite co-precipitated with calcium-doped zirconia NPs using CHFS. Rapid consolidation of the co-precipitates was again investigated using spark plasma sintering to achieve $>96 \%$ sintered densities at $1000{ }^{\circ} \mathrm{C}$ (with hydroxyapatite and calcium-doped zirconia as the only two phases). ${ }^{128}$ Mechanical tests of spark plasma sintered samples containing $10 \mathrm{wt} \%$ calcium-doped zirconia, revealed a three-pt flexural strength of 107.7 MPa and Weibull modulus of 9.9. In other related work on spark plasma sintering of CHFS made NPs of yttria stabilized zirconia (YSZ), densities of ca. $98 \%$ were achieved for YSZ and ca. $96 \%$ for $\mathrm{Ni} /$ YSZ cermets (all NiO was converted into Ni due to the reducing atmosphere of the SPS). 357 See section on 8.1 on SOFC materials for more information. The use of SPS for the consolidation of NPs is thus, very promising and is discussed in the latter part of this review as being an area for future development.

Other recent reports on surface functionalized nano-hydroxyapatite using a capping agent of DDSA, led to sufficient hydrophobic functionality to the HA surface so that it could be extracted from the water into an organic solvent. Furthermore, adding the capping agent either before or after the formation of HA (in process), did not affect the final particle morphology. If the DDSA was introduced during particle formation in the process, significantly reduced levels of free dispersant and greater thermally stability was observed, compared to samples in which DDSA was added post-nucleation in the process. ${ }^{129}$

Recently, Lester and co-workers use CHFS to fabricate three different morphologies of HA at relatively low water feed temperatures of $200^{\circ} \mathrm{C}$ or more. The addition of a $\mathrm{Zn}$ salt in the reagents also afforded a mixture of hydroxyapatite and $\mathrm{CaZn}_{2}\left(\mathrm{PO}_{4}\right)_{2} \cdot 2 \mathrm{H}_{2} \mathrm{O}$ (zinc phosphate, parascholzite). A cell study suggested that, cell proliferation was unaffected by exposure to varying concentrations of nanoparticles over the first few days. $35^{8}$ in a further study by 
the same researchers, the method was further extended to allow HA plates to be surface modified via hydrocarbon capping in the same process. ${ }^{129}$

In much of the above work, it was suggested that the calcium phosphate materials could have applications in a biomedical setting such as implantation in the body. However, it must be remembered that due to the nature of the CHFS method, the levels of trace metals that become incorporated into the bioceramics using such methods (due to minute amount of leaching from the stainless steel heated in the CHFS system) may exceed the allowed standards for such materials to gain approval for use in implants in humans. However, with use of lower temperatures or corrosion resistant flow reactors, this may be less of an issue. Therefore, more work is yet to be done in this area. Furthermore, as many of the reactions for HA can actually be made at much lower temperatures (as long as sufficient time is allowed), there also exists the possibility of using plastic flow reactors at temperatures below $100{ }^{\circ} \mathrm{C}$.

In order to reduce contaminants to the levels of international standards for the production of selected bioceramics, the UCL authors developed relatively low temperature plastic flow systems, which overcome some of these limitations. ${ }^{349-350,} 359$ In batch syntheses of phase pure calcium hydroxyapatite, the pH must not drop below 10 or else secondary phases can arise when the material is heat-treated (suggesting it's not stoichiometric Ca:P ratio of 1.67 to start with). 349 The advantage of plastic flow reactors for the synthesis of calcium phosphates such as HA is that they give good $\mathrm{pH}$ control and avoid the need for continuous $\mathrm{pH}$ adjustment and control (as required in batch syntheses of calcium apatites). In the most recent report by the authors, it was shown that surface functionalized calcium phosphates could be made using a continuous plastic flow synthesis (CPFS) system at a reaction temperature of 70 ${ }^{\circ} \mathrm{C}$ for only 5 minutes. 349

In conclusion, making bioceramics in the CHFS is very fast and allows more control over particle properties such as size, shape, crystallinity and surface area. Nano-bioceramics made via this method can be sintered into highly dense ceramic disks which excellent strength and reduced defects. In the forthcoming years, it is envisaged more studies on the potential toxicity of biological effects of such small particles in the body should be assessed before such materials gain acceptance for use. By use of careful control of nucleation and growth, it may also be possible 
to make desirable shapes of particles or properties of calcium phosphates which have the right balance of chemical, structural and biological properties.

\section{$5.4 \mathrm{ZrO}_{2}$ Biomedical Applications}

With regards to non-bioactive bioceramics, YSZ (yttria stabilized zirconia) has been widely used as a high strength sintered inert ceramic material for load bearing implants (as well extensively in dental applications because of its biological (inertness in vivo), mechanical (e.g. low wear rate) and optical properties. ${ }^{344,360}$ In one report on the batch hydrothermal synthesis of $\mathrm{ZrO}_{2}$ (from zirconium oxynitrate), when a reaction temperature of $250{ }^{\circ} \mathrm{C}$ (for $24 \mathrm{~h}$ ), researchers obtained pure monoclinic-structured $\mathrm{ZrO}_{2}$. It was found that at least $12 \mathrm{~h}$ were needed at this temperature to obtain phase pure monoclinic $\mathrm{ZrO}_{2} \cdot{ }^{361}$ In contrast, reactions conducted at the lower temperature of $200^{\circ} \mathrm{C}$ for 24 $\mathrm{h}$ were found to be mixtures of the tetragonal and monoclinic $\mathrm{ZrO}_{2}$. This suggests at these temperatures, that time is an important factor in determining the evolution of phases. ${ }^{361}$ In comparison to batch hydrothermal, there have been several reports on the use of continuous (or rapid) hydrothermal technologies for making zirconia containing ceramics, ${ }^{80,128,357,362-368}$ some of which may have potential use in biomedical applications.

In a typical continuous hydrothermal synthesis such as that reported by Hakuta et al., ${ }^{80}$ zirconia nanocrystals were prepared by hydrothermal reaction of zirconyl nitrate (and zirconyl acetate) solutions at $400{ }^{\circ} \mathrm{C}$ and $30 \mathrm{MPa}$ for a relatively short residence time of $1.8 \mathrm{~s}$. Zirconia powders had high crystallinity and a tetragonal crystal structure with a mean diameter of ca. $6.8 \mathrm{~nm}$ (using $0.05 \mathrm{M}$ zirconyl acetate solution / $0.1 \mathrm{M} \mathrm{KOH}$ ) under supercritical conditions. $^{80}$ In a later report, CHFS was used to make YSZ NPs in the temperature range 300 to $400^{\circ} \mathrm{C}$ ( $30 \mathrm{MPa}$ pressure) and in the $\mathrm{pH}$ range 1 to 11 . It was found that $\mathrm{Y}$ conversion could be increased with increasing temperature and reaction $\mathrm{pH}$. Primary particle sizes of YSZ estimated from BET surface area were in the range 4 to $6 \mathrm{~nm}$, irrespective of solution $\mathrm{pH}$ and temperature. However, secondary particle sizes measured by dynamic light scattering technique, suggested agglomeration occurred which was $\mathrm{pH}$ dependent, i.e. the surface charge was unsurprisingly greatly affected by process $\mathrm{pH}$.

One of the more interesting recent studies to manufacture small size $\mathrm{ZrO}_{2}$ and study its nucleation and growth via in situ X-ray methods was by Becker et al. ${ }^{289}$ who reported particles $<10 \mathrm{~nm}$, with narrow size distributions. The 
choice of synthesis parameters, yielded either monoclinic or tetragonal zirconia phases with a critical particle size of ca. $6 \mathrm{~nm}$ for nano-crystalline monoclinic $\mathrm{ZrO}_{2}$ under certain conditions, which was smaller than estimates (for favouring the monoclinic phase) reported in the literature. In general, a mixture of tetragonal $(t)$ and monoclinic $(m)$ phases have been obtained with higher reaction temperatures. In the report, almost phase pure $t-\mathrm{ZrO}_{2}$ was obtained at low temperature and pressure, whereas high temperature and pressure led to almost phase pure $m-\mathrm{ZrO}$ (an important catalyst). ${ }^{289}$ Others have reported particles also to generally tend to be below $10 \mathrm{~nm}$ when made in hydrothermal flow processes. ${ }^{219}$ If such particles can be dispersed and nano-sized with yttria doping in the tetragonal phase, they would be of interest as sinterable YSZ powders for implants. In one report by Galkin et al. ${ }^{369}$ on zirconia based catalysts, it was found that doping $10 \mathrm{~mol} \% \mathrm{Y}$, could also stabilize the tetragonal phase of zirconia. However, doping 10 mol\% indium, yielded mainly a monoclinic $\mathrm{ZrO}_{2}$ phase. The specific surface area of these systems was in the range 140 to $170 \mathrm{~m}^{2} \mathrm{~g}^{-1} .369$

In a report by Aimiable et al. ${ }^{156}$ (in which several other materials were reported), the researchers found that the phase of $\mathrm{ZrO}_{2}$ made in flow from zirconyl nitrate $\left(450^{\circ} \mathrm{C}, 30 \mathrm{MPa}\right)$ was strongly affected depending on the reaction medium; $t-\mathrm{ZrO}_{2}$ phase was made under normal conditions, however, under basic conditions $(\mathrm{NaOH})$ an amorphous phase was formed. This converted to orthorhombic $\mathrm{ZrO}_{2}$ after heat-treatment at $550^{\circ} \mathrm{C}$ during $6 \mathrm{~h} .{ }^{156}$ Of course, as reported elsewhere in this review, $\mathrm{ZrO}_{2} \mathrm{NPs}$ are of interest in applications such as refractive index modifiers ${ }^{118}$ (see earlier section 4.3) and as an electrolyte in SOFCs (see section 8 on solid oxide fuel cells). ${ }^{357}$ None of the publications to investigate CHFS-made pure YSZ, have looked at sintering or consolidation methods (to the best of our knowledge). Thus, given that YSZ is effectively a bioinert metal oxide, there exists significant scope to develop and exploit the high surface areas of such materials to reduce sintering temperatures (or use it as a sintering aid) in high strength biomedical implants such as high load bearing femoral heads or dental implants (subject to medical regulations). 344

\subsection{Nanophosphors/Q Dots (Bio Use)}

Continuous hydrothermal processes can deliver designer NPs with controlled size and shape and in some cases with surface functionality, which are very much of interest in biomedical labelling applications for cell imaging or tagging..$^{312}$ In the earlier sections, it was reported that nanophosphors are of interest in electroluminescent displays, however, such materials also have potential in biological imaging if appropriately made or functionalised to not elic- 
it a cytotoxic response when in use. ${ }^{214,}{ }^{217}$ In the case of the authors own endeavours in nanophosphors of europium doped yttria (red phosphor), only the corresponding yttrium hydroxide or oxyhydroxide was formed, which suggested that the reaction time and temperature used were not sufficient to fully dehydrate the product to the full oxide product. $^{217}$ However, the authors were eventually able to make crystalline and highly luminescent NP red phosphors with average particle size of $35 \mathrm{~nm}$ (nominal 4 mol\% Eu in $\mathrm{Y}_{2} \mathrm{O}_{3}$ ) using flash heat-treatment of a NP precursor (crystals of the corresponding doped oxyhydroxide). Photoluminescence (PL) and time-resolved PL measurements, were performed on selected heat-treated nanomaterials and revealed a significantly extended lifetime of $>2.25 \mathrm{~ms}$ (bulk material typically ca. $1.7 \mathrm{~ms}$ ). ${ }^{217}$ These particles were also surface-functionalized and further evaluated as probes for biological imaging (using $\operatorname{COS} 7$ cells that are a fibroblast-like cell line). Both the initial (unheated) phosphor and the highly luminescent oxide variant, were clearly resolved in cell imaging studies (see figure 15) under an excitation of $470 \mathrm{~nm}$ (using a wide pass band filter centred at $640 \mathrm{~nm}$ ). ${ }^{217}$

There have been a handful of investigations on the toxicity of CHFS-made nanophosphors with mammalian cells, e.g. Eu-doped $\mathrm{ZrO}_{2}$ made at $350^{\circ} \mathrm{C}$ or higher (> $99 \%$ conversion) was investigated for its potential toxicity towards cells. ${ }^{214}$ The structure of the NPs synthesized at $400{ }^{\circ} \mathrm{C}$ was cubic (X-ray diffraction) and the emission spectra demonstrated characteristic $\mathrm{f} \rightarrow \mathrm{f}$ transitions of $\mathrm{Eu}^{3^{+}}$ions (confirming an effective dispersion of these ions within the $\mathrm{ZrO}_{2}$ host). Furthermore, at a constant residence time in the continuous hydrothermal process, the temperature had an effect on the diameter of as made NPs in the range ca. 2 to $23 \mathrm{~nm}$ (by DLS which measures the hydrodynamic radius, so particles were actually smaller as confirmed via TEM). However, increasing the residence for a constant reaction temperature had little or no effect on size. Thus, size was largely temperature dependant in the residence time range ca. 3.7 to $7.0 \mathrm{~s}$. An initial test for the toxicity of the Eu-doped $\mathrm{ZrO}_{2} \mathrm{NPs}$ involved short ( $2 \mathrm{~h}$ ) or long (18 h) incubation of them with Chinese hamster ovary $(\mathrm{CHO})$ cells that are considered representative of a typical cell line used for live cell imaging applications. ${ }^{214}$ The cells were incubated with trypan blue dye to assess any resulting cell death due to toxicity arising from exposure to the particles (as live cells exclude trypan blue, whilst dead ones will remain stained); it was concluded from the resulting cell images that the cells were still functioning after exposure.

\section{Fig. 15}


More recently, Tang used a continuous hydrothermal process to make both coated and uncoated ZnS uncoated particles of which the 3-mercaptopropionic acid capping agent was shown to not only decrease cluster size, but also shift the isoelectric point of the particles and alter their photoluminescent properties. ${ }^{213}$ Other sulfides that have been relevant to quantum dots (Q-dots) have been made in continuous hydrothermal systems by Lester and coworkers ( $\mathrm{ZnS}, \mathrm{CdS}, \mathrm{PbS}$, etc. $)^{370}$ and thus, it may be possible that continuous hydrothermal systems can have applications for biolabelling in the future (with suitable size control and particle capping). However, to be able to compete with the performance of Q-dots made in organic solvents, may the difficult to achieve as such particles need to be very small, i.e. ca. a few $\mathrm{nm}$.

In summary, the small size of NP phosphors with the added ability to functionalize surfaces in a single process, make CHFS an attractive process for biolabelling of non-toxic alternatives to potentially toxic or Cd based biolabels.

\section{ELECTRONICS, INFORMATION AND COMMUNICATION TECHNOLOGY}

\subsection{Metals and Transparent Conducting Oxides}

Metallic Nanoparticles. Metallic nanoparticles are of interest for applications in printed electronics (for conductive tracks) or possibly for catalytic applications. NPs of copper, silver and gold (metal) are also of interest as active catalyst metals as well as for antibacterial materials (for $\mathrm{Cu}$ and Ag) ${ }^{337}$ to size dependent colourants for gold NPs and colloids. Copper and silver are also of interest for printed electronics, however, the poor stability to oxidation of some copper NPs has led to interest in developing coated copper particles and inks and solvent systems, which are more stable to oxidation. Copper (and copper oxide) particles have also been made from recovered waste acrylonitrile and copper plating water in which there is a synergy from the copper catalyzing the oxidation of the organics and the exothermal process contributing to improving the recovery of the copper..$^{77}$

In general, the authors suggest that many precious metals such as Au and Ag can be made using much milder, low temperature synthesis conditions ${ }^{303}$ and often with very narrow size distribution control, therefore, the authors do not recommend continuous hydrothermal methods as being the best routes for the direct synthesis of unsupported Au or Ag small particles. Nevertheless, both Au and Ag NPs have also been produced via the CHFS method; $;^{371-372}$ in the case of $\mathrm{Au}, \mathrm{NPs}$ were produced from precursors $\left[\mathrm{Au}\left(\mathrm{HSC}_{10} \mathrm{H}_{20} \mathrm{COOH}\right)_{n}\right]$, with no additional re- 
ductant needed (although at temperatures above $523 \mathrm{~K}$ some $\mathrm{Au}_{2} \mathrm{O}_{3}$ was produced). The optimum $\mathrm{CHFS}$ approach was to mix the Au solution with superheated water at $623 \mathrm{~K}$, which produced rounded particles up to $20 \mathrm{~nm}$ in diameter. Lester et al..$^{152}$ reported the production of Ag NPs of $9 \mathrm{~nm}$ diameter, but as yet, details of the synthesis remain unreported in the chemical literature. The authors would suggest that such chemistry is likely to require adequate quenching of the silver nanomaterials via appropriate capping agents to avoid excessive growth of agglomeration, which would reduce their effectiveness as inks. There have also been a number of reports in which metals are reduced in process in the presence of a suitable capping agent. For example, colloidal dispersions of rhodium metal (Rh) NPs have also been made using a flow process via the reduction of Rh(IIII) ions in water, ethanol, or waterethanol mixtures in the presence of poly(N-vinyl-2-pyrrolidone). At $473 \mathrm{~K}$ (25 MPa) Rh particles of $2.5 \mathrm{~nm}$ were synthesized from a solution of ethanol in which $a[R h]=15 \mathrm{mM}$. A very high dilution in water $([\mathrm{Rh}]=1.5 \mathrm{mM})$ particles with a diameter of $2.0 \pm 0.4 \mathrm{~nm}$ were possible.

In 2014, a continuous hydrothermal process was used to make surface-functionalized copper NPs (NPs) which were stable in ethanol. Copper formate, was fed into the process as an aqueous solution with polyvinylpyrrolidone (PVP) [surface modifier] and mixed with an aqueous formic acid stream, which was a source of hydrogen reducing gas in situ (reaction at $400{ }^{\circ} \mathrm{C} / 30 \mathrm{MPa}$ ). The formed surface-modified zero-valent Cu NPs were ca. $18 \mathrm{~nm}$ in size and spherical and displayed long-term (>1 year) stability in ethanol (surface plasmon resonance was used to confirm no change in size) but not in water. The stable copper NPs were used to make conductive films (resistivity of 16 $\mu \Omega . c m$ ). After the initial report, a further study was conducted to make Cu NPs (ca. $27 \mathrm{~nm}$ ) capped with PVP in flow at $400{ }^{\circ} \mathrm{C}$ and $30 \mathrm{MPa}$. These PVP-capped particles could be dispersed in distilled water, methanol, ethanol, 1propanol, 2-propanol, butanol (Figure 16). ${ }^{131}$ Ethanol, the propanols and butanol provided Cu with good oxidative protection as the PVP polymer chains formed large radii of gyration and coil-like conformations in the solvents so that they were arranged uniformly and orderly on the surface of the particles to limit oxidation in these solvents. Alternatively, when the Cu NPs coated with PVP were placed in solvents that provided poor oxidative protection (water, alcohol-water mixed solvents with 30\% water), the PVP polymer chains had globular conformations (due to more hydrogen-bonding interactions and reduced interactions with the particle surface). ${ }^{131}$ The same report also investigated the antioxidative properties of PVP-capped Cu particles.

Figure 16 
EDTA (Ethylenediaminetetraacetic acid) has also been used to cap Cu NPs. Size-controlled Cu NPs were recently prepared by a simple hydrothermal flow process. 333 The average particle size of the products increased from 14 to 50 $\mathrm{nm}$ when the copper sulfate concentration went from 0.05 to $0.5 \mathrm{~mol} . \mathrm{L}^{-1}$. Particle size distribution was broader with the increase of copper sulfate concentration. The $\mathrm{pH}$ was found to greatly affect particle size; when the molar ratio of $\mathrm{NaOH}: \mathrm{Cu}$ increased from o:1 to 2:1, average particle size significantly decreased from ca. 85 to $14 \mathrm{~nm}$. At high concentrations of EDTA in the simple flow process, particles were monodispersed due to capping of the particles. ${ }^{333}$

An interesting paper by Gadhe and Gupta, described the in situ production of Cu NPs for catalyzing the steam reformation of methanol. ${ }^{210}$ They achieved a maximum yield of around $1.2 \mathrm{~mol} \mathrm{H}_{2}$ per $\mathrm{mol}_{3} \mathrm{COH}$ (the theoretical maximum being 3), which is a highly respectable result from particles of $c a .140 \mathrm{~nm}$. This method has the disadvantage that the Cu NPs are effectively part of the waste stream and a method to recycle them would be needed.

Similar to the work on Cu metal particles, the synthesis of Ni, NPs under $20 \mathrm{~nm}$ was possible in flow via in situ reduction using a super rapid heating process with micromixer using of $\mathrm{Ni}(\mathrm{HCOO})_{2}$ solution in the presence of hydrogen at $673 \mathrm{~K}(30 \mathrm{MPa}) .{ }^{373}$ Other than this report, the authors are not aware of too many successful efforts to make $\mathrm{Ni}$ directly in process, however, Sue et al. were able to co-precipitate $\mathrm{Ni}$ with $\mathrm{Fe}_{3} \mathrm{O}_{4}$ in which the majority product was $\mathrm{Ni}$ metal. ${ }^{86}$ In the process, $\mathrm{Fe}_{3} \mathrm{O}_{4}$ was precipitated first, followed by Ni being deposited onto these particles in the presence of a reductant $\left(\mathrm{H}_{2}\right.$ from decomposition of formic acid) and 1,10-phenanthroline as an "antihydrolysis agent". In the same report, there was no detailed metal analysis data (such as energy dispersive X-ray spectroscopy using a TEM or SEM), so the exact location of the Ni was assumed to be coating the $\mathrm{Fe}_{3} \mathrm{O}_{4}$ by the fact that the $\mathrm{Fe}_{3} \mathrm{O}_{4}$ conversion and crystallite size (from XRD) are smaller than identical conditions without the Ni. This indicated that the Ni was coating on the iron oxide and thereby, terminating the growth of the latter particles. Due to their large particle size, these $\mathrm{Ni}$-coated materials were of interest for ceramic capacitor electrodes rather than as fine chemical catalysts.

In the future, the authors suggest that researchers should ask whether there are easier and milder processes that can do the job better in each case. In some cases as we have seen for copper-coated particles, the process can make very useful copper particles that are stable to oxidation to air (in the right solvent system). Future work in this area thus may focus on more aggressive approaches to in situ reduction, the use of capping agents or carbon or other coatings to prevent re-oxidation and finally, to a two-step approach that involves the synthesis of carbon-coated 
metal oxide NPs followed by carbothermal or other reduction 374 . In the latter approach, if flash heating methods are used to reduce the oxides, it may be possible to retain a metal particle size after the additional thermal processing.

Transparent Conducting Oxides (TCOs) based on Indium Tin Oxide. Transparent Conducting Oxides (TCOs) possess a combination of low resistivity and high transparency, which makes them ideal in smart windows, solar cells and in light emitting diodes (LEDs). In particular, tin-doped indium oxide (ITO) is the industry standard TCO with a typical resistivity of ca. $10^{-4} \Omega$.cm and optical transparency for thin films, typically $>80 \%$.

Given the interest in ITO as a TCO, significant research over the last two decades has focused on the development of these materials as inks and thin films. Dense thin films of ITO can be deposited using a range of techniques including chemical vapor deposition (CVD)375 sputtering and pulsed laser deposition (PLD). Some of these processes occur at high temperatures and can also involve processing steps in excess of $700^{\circ} \mathrm{C}$, which can restrict the type of substrates that can be used (e.g. is difficult to print onto plastics for flexible devices). In order to achieve high performance, TCO NPs can be used for printed coatings or tracks with control over crystallite size, morphology and structural defects (such as oxygen vacancies). Much of this depends on the NP synthesis method and conditions used.

The synthesis of ITO nanomaterials typically employs batch solvothermal / hydrothermal or co-precipitation methods. ${ }^{376}$ This includes the use of microwave-assisted solvothermal synthesis in, which some highly promising resistivities were measured. ${ }^{377}$ However, industrial scale-up of some of these processes is limited because of the use of unsuitable organic solvents (due to high Volatile Organic Compound, VOC, content) and batch-to-batch variations in processes. In one important batch hydrothermal paper for the synthesis of ITO for relatively short periods, microcrystals were successfully synthesized using $\mathrm{N}_{2} \mathrm{H}_{4}$ as a reagent. ${ }^{376}$ In the same report, the effects of various parameters and reducing agents $(\mathrm{pH}$, pressure, and temperature) on the development of ITO particles were studied. A mechanism for the evolution of the ITO via $\mathrm{In}(\mathrm{Sn}) \mathrm{OOH}$ was proposed and it was suggested that the $\mathrm{N}_{2} \mathrm{H}_{4}$ could effectively induce the depletion of oxygen in the $\ln (\mathrm{Sn}) \mathrm{OOH}$ structure (possibly via the formation of $\mathrm{H}_{2}$ and $\mathrm{NH}_{3}$ ), which accelerated the formation of cubic ITO particles with a blue colour. ${ }^{376}$ The lessons from this batch work, clearly has some use for those seeking to make phase pure ITO (free of the oxyhydroxide) in continuous hydrothermal flow reactors as will be seen later. 
Continuous Hydrothermal synthesis processes are ideal for manufacture of more sustainable TCO nanomaterials inks. Continuous hydrothermal-made NP dispersions could be suitable for screen-printing and inkjet processes, which when coupled with fast, low-temperature processing, offer a low-cost, environmentally friendly approach for the large-scale manufacture of printed optoelectronics. This includes flexible solar cell applications, where deposition on heat-sensitive, flexible or other specialized substrates is possible via low temperature sintering. In the future, as inkjet inks are becoming more water based (and less reliant on hydrocarbons or organic solvents), the continuous hydrothermal process could be envisaged as offering advantages if the materials need to be dispersed in aqueous systems.

Continuous hydrothermal methods offer the potential for controlled and consistent syntheses of TCO nanomaterials at scale. 93 A number of reports on the flow synthesis of indium oxide and doped analogues are known. For example, the synthesis of ITO by continuous processes has also been reported by Fang ${ }^{378}$ and Lu, ${ }^{379}$ however, in both these reports, $\mathrm{InOOH}$ was observed under certain conditions and reaction temperatures $>400{ }^{\circ} \mathrm{C}$ were necessary to achieve phase-pure ITO. In order to obtain sufficient conductivity in ITO nanomaterials, the extent of oxygen vacancies within the structure is known to be key to the conductivity. To achieve sufficiently reducing conditions (to make oxygen vacancies) in hydrothermal process, one approach is to use formic acid, which decomposes to form $\mathrm{CO}_{2}$ and reducing $\mathrm{H}_{2}$ gas under process conditions. This was successfully employed by Lu et al. for the CHFS production of ITO NPs (with a hexanoic acid surface coating). ${ }^{379}$ The conductivity of the as-synthesized material was $0.12 \mathrm{~S} . \mathrm{cm}^{-1}$ after pressing (9.6 MPa) into compacts of density ca. $3.6 \mathrm{~g} . \mathrm{cm}^{-3}$. This ability of CHFS to facilitate in-process surface functionalization of ITO NPs, may be a useful general approach to making high quality and organic solvent (or water) stable inks in the future.

Whilst the doping of indium oxide with $\mathrm{Sn}$ is needed to form a working TCO, CHFS has been used for the direct synthesis of undoped cubic $\ln _{2} \mathrm{O}_{3}$ (bixbyite) NPs, 93 including for gas sensing applications ${ }^{380}$. More recently, the UCL authors reported the synthesis and characterization of ITO made by CHFS (followed by a heat-treatment), using $\left[\mathrm{In}\left(\mathrm{NO}_{3}\right)_{3} \cdot \mathrm{H}_{2} \mathrm{O}\right]$ and $\left[\mathrm{K}_{2} \mathrm{SnO}_{3} \cdot{ }_{3} \mathrm{H}_{2} \mathrm{O}\right]$ precursors. 93 The influence of $[\mathrm{KOH}]$ and formic acid concentration, on the phase and performance of the materials was studied, as well as electrical resistivity vs tin concentration (using pressed and fired monoliths). Tin-doped indium oxide (known as ITO) NPs with up to 12 at\% Sn, were synthesized at a process rate of $c a .15 \mathrm{~g} \cdot \mathrm{hr}^{-1}$, followed by heat-treatment (inert atmosphere). The use of formic acid in the CHFS process was found to enhance the conductivity of powders that were pressed and heat-treated into pellets. Resistivity values as 
low as $6.0 \times 10^{-3} \Omega . \mathrm{cm}$ were obtained for a sample with a In:Sn ratio of $9: 1$, which is superior to the best ITO pressed powders reported in the literature to date. ${ }^{93}$ However, as much of the conductivity was effectively limited by grain boundary resistance effects of the NPs, the authors expect that higher conductivities could be achieved if the powders could be printed in thin film form using the disks as sputter targets or other printable inks. Following optimization of the dopant concentration within the ITO system, scale-up production of the materials was carried out on a volumetric basis by means of an increase in flow rate on a pilot-plant continuous hydrothermal reactor. Materials produced at a higher rate of production $\left(c a .100 \mathrm{~g} \cdot \mathrm{h}^{-1}\right)$, showed similar primary particle sizes and morphologies to those produced of the lab-scale, with a comparable resistivity value (for a heat-treated disk) of $9.6 \times 10^{-3} \Omega . \mathrm{cm} .{ }^{93}$ see figure 17 .

Figure 17 .

Recently, Adschiri and co-workers reported the synthesis of tin and zinc co-doped indium oxide (IZTO) with a mean diameter of ca. $28 \mathrm{~nm}\left(45^{\circ} \mathrm{C}, 30 \mathrm{MPa}\right)$. When the molar ratio of $\mathrm{In}: \mathrm{Sn}: \mathrm{Zn}$ was 9:1:0, the product was found to be the single phase of cubic $\ln _{2} \mathrm{O}_{3}$ and no other peaks were found. ${ }^{381}$ When the molar ratio of $\ln : \mathrm{Sn}: \mathrm{Zn}$ was $8: 1: 1$, the phase pure $\ln _{2} \mathrm{O}_{3}$ crystal structure was also formed. Conversely, in the case where the molar ratio of In:Sn:Zn was set to 6:2:2, a cubic $\ln _{2} \mathrm{O}_{3}$ structure was obtained with traces of $\mathrm{SnO}_{2}$. The same researchers added hexanoic acid into the precursors to yield surface-modified IZTO that was obtained as $<20 \mathrm{~nm}$ particles, which could be dispersed in organic solvents. ${ }^{81}$ The conductivity and transmittance of these IZTO nanocrystals were also measured and the conductivity in was found to be in the range ca. 0.2 to $9.0 \times 10^{-3} \mathrm{~S} . \mathrm{cm}^{-1}$ depending on composition and pressure for the disks that were measured. ${ }^{381}$

Recently, some of the UCL authors reported that ITO thin films of ITO could be prepared on glass substrates by dip and spin coating methods. ${ }^{382}$ Aqueous dispersions of "ITO nanopowder" (made via continuous hydrothermal synthesis) were prepared and assessed for stability in a range of surface agents, e.g. PEG 400, Tween 80 and $\beta$ alanine. Both PEG 400 and $\beta$-alanine were found to produce stable dispersions that could be used to form good quality films after heat-treating, using both conventional and microwave heat-treatment. ${ }^{82}$ All the films exhibited an average transmittance of $>80 \%$ over the visible spectrum. Furthermore, ITO films prepared with no dispersant showed very high resistivity values for both heating methods, however, addition of 2 wt\% PEG 400 to the dispersion, 
yielded a reduction in the resistivity values to $1.4 \times 10^{-1} \Omega . \mathrm{cm}$ and $3.8 \times 10^{-2} \Omega . \mathrm{cm}$ for conventionally and microwave treated films, respectively. The surface morphological studies confirmed that addition of dispersants improved the film uniformity and inter-particle connections of the ITO films considerably. ${ }^{382}$

More Sustainable TCOs. Given the increasing cost and scarcity of indium, there is a need to find alternative, more sustainable alternative elements for TCOs, e.g. based on $\mathrm{ZnO}^{375}$ or $\mathrm{TiO}_{2}$ hosts. Both these host materials have been synthesized extensively in their undoped form using CHFS. ${ }^{36,120}$ The synthesis of ZnO NPs doped with Al (AZO), Ga (GZO), as well as co-doped with both Al and Ga (AGZO), has been investigated by analogous methods to that used for ITO (note: gallium oxide is not a TCO on its own, its synthesis in a flow type tubular reactor was reported by Anikeev $\left.{ }^{383}\right)$

The optimal composition of aluminum in $\mathrm{AZO}$ was found to be 2.5 nominal at $\% \mathrm{Al}$ (with respect to 97.5 at $\% \mathrm{Zn}$ ) in the precursor solution; the synthesized material was found to have a resistivity of $7.0 \times 10^{-3} \Omega . \mathrm{cm}$, comparable to the observed for ITO synthesized and processed by the same methods. A further increase in dopant composition led to an increase in the resistivity values obtained. Gallium-doping yielded a slightly higher resistivity value of $9.1 \times 10^{-}$ ${ }_{3}^{3} \Omega . \mathrm{cm}$ at an optimum dopant concentration of 3.5 at $\% \mathrm{Ga}$. Further increase in dopant concentration, had little effect on the electrical properties of the material. ${ }^{384}$ Co-doping of Al and Ga up to a total dopant concentration of 5 at $\%$ dopant (at increments of 0.5 at $\%$ for the individual dopants), was also investigated by the authors. ${ }^{85}$ This yielded a ternary contour plot of compositional space with respect to the resistivity values. 'Islands' of optimum conductivity were identified within the compositional space, in particular those observed at Zn:Al:Ga ratios of 96:2:2 and 97:1:2, yielded resistivities of $1.0 \times 10^{-2}$ and $9.1 \times 10^{-3} \Omega . c m$, respectively. The rate of production for each AZO, GZO and AGZO was $60 \mathrm{~g} \cdot \mathrm{hr}^{-1}$. Upscaling of GZO synthesis to a rate of $300 \mathrm{~g} \cdot \mathrm{hr}^{-1}$ on a pilot-scale continuous hydrothermal process, resulted in reduced uptake of $\mathrm{Ga}$ into the $\mathrm{ZnO}$ structure due to a reduced residence time, however with 4.5 at $\%$ Ga in the precursor solution, resistivity of the product was comparable to the laboratory scale process used above. The use of formic acid in synthesis of AGZO was also investigated; this material demonstrated resistivities of $4.6 \times 10^{-2} \Omega . \mathrm{cm}$, indicating that though providential in the synthesis of ITO, formic acid did not have a similarly positive effect in the synthesis of $\mathrm{ZnO}$-based nanomaterials in terms of the electrical properties.

Further scale-up of ZnO-based TCO nanomaterials has also been demonstrated by the authors. Synthesis of $\mathrm{AZO}(2.5 \mathrm{at} \% \mathrm{Al})$ at total metal concentrations in the range $0.2-1.0 \mathrm{M}$ on a pilot-scale CHFS reactor, showed a small 
increase in mean particle length and aspect ratio with increasing concentration, in agreement with observations made for a similar mass-based scale up of undoped $\mathrm{ZnO} .{ }^{170}$ The resistivities of the materials showed little variation across concentration scales, with values measured in the range $5.1 \times 10^{-2}$ to $9.2 \times 10^{-2} \Omega . \mathrm{cm}$. The lower resistivities of materials produced on the pilot-scale compared to those produced by lab-scale CHFS, was attributed to their smaller particle size, resulting in greater grain boundary resistance and higher resistivity of the bulk material.

For the formulation of TCO NP inks for thin film deposition, in-process surface functionalization of AZO NPs by CHFS has been by the UCL authors where it was possible to introduce citric acid (CA) capping agents. Such functionalized nanomaterials can be formulated into inkjet printable inks.

Thus, there appears to be considerable opportunities to improve on current TCO inks for device fabrication and also to rapidly discover new materials and dopants to eventually replace ITO as a TCO. In the future, in order to maximise success, experimentalists and modellers ${ }^{36-392}$ should work in closer collaboration in order to not only discover but also to better understand newly proposed TCOs based on low cost, more sustianable and less toxic materials.

\subsection{Semiconductors (Electronics and Gas Sensors)}

Semiconductors are substances that are neither good conductors (metals) nor insulators (glass) and can take a wide variety of forms, however in the context of CHFS processes this will largely concern doped or undoped metal oxides or other metal compounds. Semiconductors are important for use in electronics, solar cells and gas sensors or other applications. In many applications when semiconductors are incorporated into a device, usually some external influence (light, the presence of a redox gas, etc.) can cause changes in resistance or electronic properties (in a certain temperature range of operation), which can be measured or exploited for a purpose, e.g. sensing, energy harvesting, switching, etc.

Semiconductor Electronics. Many semiconductors are the basic building blocks of modern electronic circuits, including digital and analog integrated circuits, solar cells, light-emitting diodes (LEDs) and transistors. NiO made via CHFS was investigated by Takami et al. as a channel material in a field-effect transistor. ${ }^{393}$ The hydrothermal flow system yielded plates of thickness of ca. $10 \mathrm{~nm}$ and a lateral particle size in the range 100 to $500 \mathrm{~nm}$. It was suggested that the plate-like structure was retained from the intermediate $\mathrm{Ni}(\mathrm{OH})_{2}$ that would have first formed. The plates 
were purified and drop-cast on a bottom-gate substrate and used as the channel material in a field-effect transistor (after annealing at $300^{\circ}$ C). 393 The current-voltage profile was measured and showed that the nanoplates worked as a the p-type semiconductor. 393 In comparison, the UCL authors precipitated NiO directly via a CHFS process. NiO was prepared using superheated water inlet temperature of $450^{\circ} \mathrm{C}$ and pressure of $24.1 \mathrm{MPa}$, with an additional band heater set at $450^{\circ} \mathrm{C}$ on a counter-current mixing point. ${ }^{136}$ The band heater was critical to maintain a high reaction temperature so that pure $\mathrm{NiO}$ was formed (rather than a co-mixture with the hydroxide). TEM images of this sample showed that extremely small ca. $3 \mathrm{~nm}$ (average) particles were formed (range 2 to $8 \mathrm{~nm}$ ) with a BET surface area of $167 \mathrm{~m}^{2} \mathrm{~g}^{-1}$. It will be shown later on the average temperature upon mixing of the hot water and the metal salt feeds is indeed crucial to not only obtaining high yields, but also obtaining small nuclei with narrow particle size distributions (although this also depends on solubilities to some extent also). To some extent, the actual reaction temperature (where nucleation occurs) is a product of the type of mixer used as well as of course the reaction parameters of relative flow rates and inlet superheated water inlet temperature in particular.

Other materials that might be of interest in printed electronics from nanoparticles are tungsten oxides that are wide band gap semiconductors that are used in electrochromic devices and have switchable optical properties. $394-396$ Although such materials made via CHFS methods have not been tested in device applications, it is possible to manufacture them in flow reactors, however, this has required the use of an alkoxide precursor tungsten (VI) ethoxide. ${ }^{397}$ It was found that when a solvothermal (ethanol) flow synthesis route was used an amorphous product was formed. However, using high temperature water feed at $450^{\circ} \mathrm{C}$ (corresponding to an actual temperature after mixing of near to $330^{\circ} \mathrm{C}$ ), $\mathrm{WO}_{3}$ nanoplates ( $93 \%$ hexagonal phase and $7 \%$ triclinic phase) were generated. 397 The reduction temperature of $\mathrm{WO}_{3}$ nanoparticles to tunsten metal was measured and the onset of reduction was found to be much lower $\left(<200^{\circ} \mathrm{C}\right)$ than that for the corresponding bulk tungsten trioxides, suggesting that such materials may be interesting for the industrial production of metallic tungsten.

Gas Sensor Nanomaterials. A limited number of semiconducting nanomaterials made via CHFS have been investigated for gas sensors. The principles of such sensors effectively lie in the exposure of heated electrodes to various gases, which interact to either remove or add electrons, resulting in changes in resistivity. Some of the authors reported the fabrication of gas sensors by screen printing semiconductor ceramic inks onto an alumina substrate with interdigitated gold contacts (Figure 18a). Each layer was dried under an infrared lamp, burning off the organic vehi- 
cle in a furnace. Subsequently, the sensors were spot-welded with platinum wire onto a housing cap that allowed it to fit into a gas sensing rig chamber. The first reported testing of a gas sensing nanomaterial made via CHFS was for indium oxide..$^{380}$

Bulk indium oxide $\left(\ln _{2} \mathrm{O}_{3}\right)$ is a wide bandgap semiconductor $\left(\mathrm{E}_{g}=3.7 \mathrm{eV}\right)$, well-known for its useful optoelectronic properties and as a chemical gas sensor in the doped and undoped forms (particularly sensitive to reducing gases such as ethanol and ammonia and oxidizing gases such as ozone..$^{39}$ The most widely accepted theory of metal oxide semiconductor gas sensing states that "when a metal oxide semiconductor gas sensor is exposed to air, oxygen species are adsorbed on the surface of the sensor material and go on to be ionized by electrons from the materials conduction band to form species such as $\mathrm{O}_{2}{ }^{-} \cdot 399$ As such in air, the measured resistance of $n$-type semiconductors will increase due to the lower concentration of free electrons in the conduction band of the material. Surface reactions between this oxygen species and an analyte gas, may also occur on exposure to a reducing gas (such as ethanol or $\mathrm{NO}_{2}$, see figure $\left.18 \mathrm{~b}^{120}\right)$. This may lead to the release of electrons trapped in the previously ionized oxygen species, back into the material's conduction band, thus, lowering the measured resistance.

Semiconductors such as $\ln _{2} \mathrm{O}_{3}$ crystallites with reduced dimensions, can significantly increase sensor performance, for example, Korotecenkov et al. reported $>103$ fold increase in response to ozone at $300^{\circ} \mathrm{C}$, with a concomitant drop in response time, when the average $\ln _{2} \mathrm{O}_{3}$ primary particle size was reduced from 80 to $15 \mathrm{~nm} .{ }^{400}$ Most syntheses of $\ln _{2} \mathrm{O}_{3}$ NPs have involved batch solvothermal or hydrothermal methods, which are lengthy, often requiring between 12 and $24 \mathrm{~h}$ to complete. These reactions sometimes lead to formation of $\ln (\mathrm{OH})_{3}$, which then requires heat-treatment at $>400^{\circ} \mathrm{C}$ in order to convert it to $\ln _{2} \mathrm{O}_{3}$, resulting in particle growth, which can reduce responsiveness and sensitivity of the final gas sensor. The $\ln _{2} \mathrm{O}_{3}$ particles made via CHFS were well-defined (phase pure cubic bixbyite), with the majority appearing as rounded cubes in the size range 5 to $20 \mathrm{~nm}$ (see Figure $18 \mathrm{c}$ and $18 \mathrm{~d}$ at different magnifications). ${ }^{380}$ The CHFS-made $\ln _{2} \mathrm{O}_{3}$ gas sensors had an almost square response shape observed for ethanol, which suggested that the sensors rapidly responded to ethanol. When the ethanol flow was stopped, the response dropped to near baseline levels (indicative of a lack of surface sites suitable for reaction, i.e., that the resistance of the sensor was surprisingly dominated by the bulk contribution). ${ }^{80}$ It was found that the devices exhibited selectivity towards ethanol and were significantly more responsive to this gas other literature sensors using $\ln _{2} \mathrm{O}_{3}$ giving a response (known as $\mathrm{R}_{\circ} / \mathrm{R}$ where $R_{\mathrm{o}}$ is resistance at the point immediately prior to exposure to ethanol and $R$ is the resistance when exposed to ethanol). ${ }^{80}$ This shows a valuable application for continuous hydrothermal syn- 
thesis in which the NPs can be made directly with a high surface area in flow due to the high synthesis temperature, giving a literature-leading gas sensor response for ethanol. In order to test cross-sensitivity, the $\ln _{2} \mathrm{O}_{3}$ gas sensors were also tested against $\mathrm{NO}_{2}, \mathrm{CO}$, and butane at a variety of different sensor operating temperatures. ${ }^{80}$ Analysis by $\mathrm{XRD}$ and Raman spectroscopy indicated that the nano-size was retained after sensing. Interesting to note is that upon the addition of dopants (such as for transparent conducting oxides), the oxy-hydroxide phase was formed under similar conditions (see transparent conducting oxides section)..$^{380}$

Figure 18

Semiconductor $\mathrm{ZnO}$ nanostructures have also received attention for gas sensing in the literature.401-402 A single step synthesis method was used for the synthesis of crystalline $\mathrm{ZnO}$ nano-rods and nano-prisms via a pilot plant scale-CHFS. ${ }^{120}$ The size and shape of the as-prepared $\mathrm{ZnO}$ nanostructures were affected by the amount of peroxide and zinc precursor used in this CHFS (peroxide is known to give bigger particles by increasing solubility of the $\mathrm{Zn}$ salt). The synthesis of both $\mathrm{ZnO}$ rods and prismatic crystallites as a function of zinc precursor concentration was observed and the depletion of $\mathrm{Zn}^{2+}$ ions in solution resulted in sharpening of the rods. ${ }^{120}$

The $\mathrm{NO}_{2}$ gas sensing properties of as-prepared $\mathrm{ZnO}$ nanostructures was carried out; as the ability of the sensor material to absorb and ionise oxygen species was key to the sensor performance, good performance was likely to be due to the reduced crystallite size, making surfaces more reactive and likely to absorb oxygen (and form ionized oxygen species). ${ }^{120}$ The $\mathrm{ZnO}$ NP sensors were tested against $\mathrm{NO}_{2}$ gas at a variety of concentrations and operating temperatures..$^{120}$ The highest gas response of 128 was observed at an operating temperature of $350{ }^{\circ} \mathrm{C}$ towards 10 ppm NO $\mathrm{N}_{2}$ gas. This compared favorably to other reports of nanoscale $\mathrm{ZnO}$ gas sensors in the literature. The sensors made by the authors gave a gas response of over 80 when exposed to $4 \mathrm{ppm} \mathrm{NO}_{2}$. This compared with a gas response of 3 for literature $\mathrm{ZnO}$ nanotubes exposed to $5 \mathrm{ppm} \mathrm{NO}_{2}$ gas, a gas response of 50 or 60 towards $5 \mathrm{ppm} \mathrm{NO}_{2}$ gas for $\mathrm{ZnO}$ nanowires ${ }^{403-404}$ or a gas response of 4 towards $8.5 \mathrm{ppm} \mathrm{NO}_{2}$ gas for a $\mathrm{ZnO}$ based nano-belt sensor. Note: the optimal operating temperature for these sensors was $350^{\circ} \mathrm{C}$ and is somewhat higher than other reports where it is typically $225^{\circ} \mathrm{C}$ or so.

6.3 Dielectrics, Piezoelectrics, Ferroelectrics and Multiferroics 
Continuous Hydrothermal synthesis reactors have made a number of potential dielectrics, piezoelectrics, ferroelectrics and multiferroics. In many cases, nanomaterials were made, but only tested for one or none of these applications. A good deal of the literature in this area concerns the manufacture of heterometallic oxides including certain perovskites for example, and therefore, it is a challenge to make such materials directly in hydrothermal flow. Before a discussion on specific materials, we will consider what properties and applications such materials possess.

Delectric and piezoelectric ceramics represent advanced commercial materials that as a result of their relatively poor electrical conductivity, have found applications in electrical charge storage or energy scavenging / conversion devices. ${ }^{405,406}$ Capacitors are essential components in electronic circuits that store energy in the form of an electric field generated between two separated, oppositely charged plates. Their capacity can be increased by using a solid dielectric material between these plates. In comparison, piezoelectrics are materials that generate a voltage when mechanical pressure is applied. ${ }^{406}$ Furthermore, they can show a change in dimensions when subjected to an electromagnetic field. Some piezoelectric materials are made of the same ceramic material as capacitor dielectrics.

In order to better understand ferroelectricity and its origins, ${ }^{407}$ we can consider one of the most well-known ferroelectric materials, $\mathrm{BaTiO}_{3}$, which has a face centred cubic (fcc) structure. Above a certain temperature $\mathrm{BaTiO}_{3}$ undergoes a phase transition to tetragonal, resulting in a permanent dipole. This spontaneous polarization is known as ferroelectricity; the temperature below which the polarity is exhibited is called the Curie temperature or point. As polycrystalline materials consist of randomly oriented domains, there is overall cancellation of the polarization. However, if an electric field is applied (such as in the case for a working capacitor), some adjacent domains begin to align with the field, producing large net polarizations. The susceptibility of these materials to electric polarization is directly related to their capacitance, i.e. their ability to store electric charge. Many of the ferroelectric materials (including some perovskites) are also piezoelectrics. ${ }^{407}$

Amongst the most important applications of thin-film ferroelectrics is in random-access memories (RAMs) for computers, including non-volatile ones in which memory is retained even when power is switched off (known as ferroelectric random-access memories, FERAMs). ${ }^{408}$ In FERAMS, the opposing directions of polarization are effectively, two states of binary logic, i.e. 1. or o. Because of their larger dielectric constants, titania-based ferroelectrics can achieve higher bit densities than more conventional silica-based semiconductors when used as thin-film capacitors in dynamic random-access memory (DRAM) applications. ${ }^{408}$ 
Multiferroics is the term used to describe a class of advanced multi-functional materials, which display simultaneous magnetic spin, electric dipole, and ferroelastic ordering and are of interest for a variety of device applications due to their multi-functionality. $4^{09}$ If the magnetic and ferroelectric orders occur independently, the multiferroic material may be denoted as type I. If the ferroelectric and magnetic transitions emerge jointly, the multiferroic is of type II. As such single-phase multiferroic materials exist rarely in nature, there are considerable attempts to discover new ones or to design new engineered materials with strong magneto-electric (ME) coupling. A more detailed review on multiferroics by Fiebig et al. is recommended for further reading. 409

Dielectrics: Materials with high dielectric constant (high- $k$ materials) play an increasingly important role in electronic devices. New dielectric materials with higher dielectric constants are required to develop ever more miniaturised devices for the next generation of high-performance transistor devices, capacitors as well as processors. Recently, progress in making high quality dielectric ceramics, particularly developing structure-property-composition relationships for new materials with high permittivity, better temperature stability and considerably better sintering ability, to develop low temperature co-fired multilayer devices possible.410-413 Due to these demands, finer particles are needed to make a progressive multilayer condenser with high densification and high capacity.

Industrially, dielectric ceramics have been produced by batch processes, such as co-precipitation, ${ }^{414-416}$ sol-gel processes, ${ }^{417-419}$ solvothermal syntheses, ${ }^{420-421}$ plasma chemical vapour deposition (CVD) ${ }^{40,422}$ or other approaches. Since hydrothermal flow synthesis has been shown to be one of the best methods for producing nanoceramics ${ }^{197}$, a worldwide research interest has been stimulated in this a clean and fast way to these materials.

In one of the early studies for the flow synthesis of $\mathrm{BaTiO}_{3}$ by Pommier et al. in $1999,{ }^{423}$ a semi-continuous twostep system was used that combined liquid phase hydrolysis of a mixed Ba-Ti alkoxide in an alcohol water mixture (T range of 150 to $230{ }^{\circ} \mathrm{C}$ and pressure ca. $\left.10 \mathrm{MPa}\right)$, followed by thermal treatment under supercritical conditions $(\mathrm{T}=$ ca. $300^{\circ} \mathrm{C}$ ). This process was successful in obtaining particles that were generally $<50 \mathrm{~nm}$ in size and when sintered at high temperatures of $1250^{\circ} \mathrm{C}$, the grains were ca. 1 micron in size, which is relatively small and usually ideal for obtaining materials with a high dielectric constant. ${ }^{423}$

A different alkoxide based approach for the synthesis of barium titanate NPs was used with various Supercritical Fluids (water in ethanol, or water) using a continuous reactor ${ }^{203}$. Liquid titanium iso-propoxide and powdered barium iso-propoxide, were used as starting materials. The researchers found that water to precursor ratio, reaction 
temperature and the Ba:Ti molar ratio, played a major role in the crystallization of the NPs. Meanwhile, wellcrystallized $\mathrm{Ba}_{x} \mathrm{Sr}_{1-x} \mathrm{TiO}_{3}$ was synthesized over the entire range of compositions, under supercritical conditions..$^{424}$ The mean particle size of monocrystalline NPs was ca. $20 \mathrm{~nm}$ in the case of Sr-rich powders and $40 \mathrm{~nm}$ in the case of Ba-rich powders. The composition of $\mathrm{Ba}_{0.7} \mathrm{Sr}_{0.3} \mathrm{TiO}_{3}$ appeared to display promising dielectric characteristics. $4^{24}$ As will be mentioned elsewhere, the early work by Pommier in supercritical alcohol/water mixtures, has been extended by Aymonier and co-workers, who have reported similar reactions in flow reactors (or in very fast processes), e.g. for in situ studies in barium titanate zirconates, ${ }^{425}$ sub-20 $\mathrm{nm}$ barium titanate zirconates over the whole solid solution range. ${ }^{426}$ Many of these papers are in more detail elsewhere in this review.

Hakuta et al. ${ }^{81}$ investigated the continuous hydrothermal synthesis of barium titanate under sub and supercritical conditions, from mixture of barium hydroxide $(0.1 \mathrm{M})$ and titanium dioxide $(0.12 \mathrm{M})$ as starting reagents at high $\mathrm{pH}$ condition $(\mathrm{pH}>13)$. Experiments were performed within the temperature range of 300 to $420^{\circ} \mathrm{C}$ and pressure of 20 to $40 \mathrm{MPa}$, corresponding to significantly different water densities. The researchers found that cubic phase $\mathrm{BaTiO}_{3}$ could only be made under higher water density (subcritical condition), whereas the tetragonal phase, could be easily obtained under supercritical conditions. Furthermore, the effect of reaction temperature and time on particle size and size distribution at constant pressure, was reported $i^{82}$ an increase in temperature in the range 300 to $380{ }^{\circ} \mathrm{C}$, led to a decreased in size from 46 to $36 \mathrm{~nm}$ (with narrower size distribution). However, at 400 and $420{ }^{\circ} \mathrm{C}$, the reaction was complete within only few seconds and highly tetragonal crystalline $\mathrm{BaTiO}_{3}$ particles of ca. $50 \mathrm{~nm}$ (average) were obtained, with a wide size distribution. By measuring the $\mathrm{TiO}_{2}$ precursor conversion into $\mathrm{BaTiO}_{3}$, they concluded that higher $\mathrm{TiO}_{2}$ conversion occurred at lower flow rates and higher temperatures.

In a further report on the synthesis of $\mathrm{BaTiO}_{3}$, the tetragonality of $\mathrm{BaTiO}_{3} \mathrm{NPs}$ increased with increasing the temperature and pressure, because at higher temperatures, dehydration rates increased and at higher pressure, the products were more crystalline. Figure 19 summarizes the relationship between synthesis temperature and pressure (water density) and the crystal phase. ${ }^{83}$ The same researchers suggested that when fine $\mathrm{BaTiO}_{3} \mathrm{NPs}_{\text {sere }}$ prepared by CHFS $\left(400^{\circ} \mathrm{C}\right.$ and $30 \mathrm{MPa}$ ) from barium hydroxide and titanium dioxide as starting precursors, a reasonably high $\mathrm{pH}$ was needed to dissolve the titania, which then reacted via a dissolution-recrystallization process for the synthesis of $\mathrm{BaTiO}_{3}$ NPs. 53,427 
Hayashi and coworkers used a continuous hydrothermal process at $400{ }^{\circ} \mathrm{C}(30 \mathrm{MPa}$ ) for the synthesis of cubic phase $\mathrm{BaTiO}_{3}{ }^{428} \mathrm{NPs}$ using a $\mathrm{TiO}_{2}$ sol (or $\mathrm{TiCl}_{4}$ ) and barium nitrate (aq.) in a single mixed solution. This was added to $\mathrm{KOH}$ solution in flow before being mixed with the sc-water flow..$^{28}$ In a modified system, Hayashi and co-workers, made cubic barium titanate $\left(\mathrm{BaTiO}_{3}: \mathrm{BT}\right) \mathrm{NPs}$ coated with surface agents using a $\mathrm{TiO}_{2}$ sol, albeit with barium nitrate as the Ba source and $\mathrm{KOH}$ to increase the $\mathrm{pH}$ in flow ${ }^{429}$ (for more details, see section 10.2).

More recently, barium strontium titanate, $\mathrm{Ba}_{x} \mathrm{Sr}_{1-x} \mathrm{TiO}_{3}$, nanopowders were made at lab and pilot-scale from mixed solutions of the corresponding Group 2 metal nitrates with TiBALD [titanium bis (ammonium lactato) dihydroxide], using a large excess of base $(\mathrm{NaOH}) .430$ The products were sub-stoichiometric, which was due to the side formation of the metal carbonates (can be removed with acid wash). It was found that increasing strontium content, led to a reduction in particle size and lattice parameters (as would be expected by Vegards Law). 430 The impure products were made at the pilot-scale of $80 \mathrm{~g} \cdot \mathrm{h}^{-1}$ without using a $\mathrm{TiO}_{2}$ particle source feed (as opposed to a soluble feed of TiBALD). It was suggested that increasing the residence time and or degassing the precursors might have been sufficient to promote a useable product. Therefore, evidently this work will require further investigation in future to be of use to industry.

Other structures, which and may be of interest as dielectrics (or potentially as cathodes in solid oxide fuel cells) include partially substituted perovskite oxides similar to $\mathrm{Ca}_{0.8} \mathrm{Sr}_{0.2} \mathrm{Ti}_{0.9} \mathrm{Fe}_{0.1} \mathrm{O}_{3-x_{.}} 431-433$ The samples were formed using a residence time of less than $10 \mathrm{~s}$ to give an average particle size of $20 \mathrm{~nm}$ and BET specific surface area of ca. $71 \mathrm{~m}^{2} \mathrm{~g}$ ${ }^{1.431}$ This is a very high surface area for such a perovskite material and certainly puts CHFS-technology at the forefront of processes to make high surface area versions of such materials directly in process.

$\mathrm{TiO}_{2}$ based materials (e.g. $\mathrm{TiO}_{2}-\mathrm{Bi}_{2} \mathrm{O}_{3}$ ) ceramics, exhibit a comparatively high dielectric constant in the lowerfrequency band. 434 For example, the composition of $0.92 \mathrm{TiO}_{2}-0.081 \mathrm{Bi}_{2} \mathrm{O}_{3}$ has a high dielectric constant $\left(\varepsilon_{\mathrm{r}}=80\right)$, high $\mathrm{O}(1800$ at $5 \mathrm{GHz})$ and low temperature coefficient of resonant frequency $\left(\tau_{f}=+21 \mathrm{ppm} \cdot{ }^{\circ} \mathrm{C}^{-1}\right) .434$ The phase mixture was identified as $\mathrm{TiO}_{2}$ and $\mathrm{Bi}_{2} \mathrm{Ti}_{4} \mathrm{O}_{11}$ these two phases possess a high $\varepsilon_{\mathrm{r}}$ with a negative temperature coefficient and a high $\varepsilon_{\mathrm{r}}$ with a positive temperature coefficient, respectively. 434

NP $\mathrm{TiO}_{2}$ may also be suitable for use in dielectric Low Temperature Co-fired Ceramic (LTCC) materials. A LTCC material is one in which the ceramic layers are co-sintered along with metal contact layers to form a component in a single step. ${ }^{435-436}$ As the most commonly used contact metal in this application is silver, the ceramic must be fired 
and have useful microwave properties $<961{ }^{\circ} \mathrm{C}$, which is the melting point of silver. No doubt this will be an area, which is in need of development in the future for CHFS-made nanoceramics.

Ferroelectrics and Multiferroics. Ferroelectrics and multiferroics are a class of materials that exhibit switching of physical properties under an external influence of some kind. ${ }^{409,437}$ As explained earlier, ferroelectrics demonstrate a switchable electric polarization when an electric field is applied. This compares to multiferroics that exhibit a similar 'ferroic' behaviour in two or more of their properties (usually electric, magnetic or elastic).

The development of superior nano-grained dense ferroelectrics materials is now an active area of interest, one in which Aymonier and colleagues have shown many examples in which manufacture of such materials in continuous Supercritical Fluids processes, can be useful as a feed powder for sintering.426, 438-439 They have also reviewed the pros and cons of synthesis methods for ferroelectric materials based on $\mathrm{BaTiO}_{3}$ (an important material that is used in the electronic industry due to its outstanding dielectric, piezoelectric and ferroelectric properties) and compared them to materials made under supercritical conditions. 438 Notably, the ferroelectric properties of $\mathrm{BaTiO}_{3}$ materials depend on defect concentrations such as the density of oxygen vacancies (this can be probed via reduction experiments by changing the oxygen partial pressure). Related factors, which also need to be considered, include the possible cation charge compensation mechanisms such as cation vacancies or changes in charge, e.g. $\mathrm{Ti}^{4+}$ or $\mathrm{Ba}^{2+}$ vacancies or $\mathrm{Ti}^{3+}$ species towards charge balance of the $\mathrm{O}^{2-}$ vacancies. $4^{40}$

Ferroelectric materials are widely used in energy harvesting systems and sensors and include the family of piezoelectric materials such as lead zirconate titanates (PZT).407, 440 Replacements for the leading material PZT, are required as environmental restrictions on the use of lead are coming into force. One of the possible lead free PZT replacements is $\mathrm{KNbO}_{3}$ in which the substitutions of $\mathrm{Na}$ for $\mathrm{K}$ and Ta for $\mathrm{Nb}$ can give favourable ferroelectric properties. The development of nanopowders of such materials are of interest because they are more conventionally made using solid state methods in excess of $1000{ }^{\circ} \mathrm{C}$ for between 2 to $4 \mathrm{~h}$ or so..$^{\circ}$ Nanopowders should also be sinterable at lower temperatures, which will be important for production of electromechanical applications (e.g. sensors or energy harvesting). Accurate control of residual defect density is also required to allow use of reproducible ferroelectric materials (in order to better understand their properties). The use of solid state routes, as well as defect chemistry for improving current processing routes to ferroelectrics, including breakthrough approaches, have been reviewed recently by Aymonier and colleagues. 440 
Reports of continuous syntheses of ferroelectrics, include the work of Hayashi and co-workers who made alkali niobate NPs such as $\mathrm{K}_{1-x} \mathrm{Na}_{\times} \mathrm{NbO}_{3}$ ( (size range ca. 50 to $60 \mathrm{~nm}$ ); these particles were made in the temperature range 400 to $480{ }^{\circ} \mathrm{C}$, (pressure range 25 to $30 \mathrm{MPa}$ ) with residence times in the range 0.1 to $8.3 \mathrm{~s}$. Single phase $\mathrm{KNbO}_{3}$ was obtained at $480^{\circ} \mathrm{C}$ and $25 \mathrm{MPa}$ at an initial $\mathrm{K} / \mathrm{Nb}$ molar ratio of $15^{30}$ The $\mathrm{K}_{1-x} \mathrm{Na}_{\times} \mathrm{NbO}_{3}$ nanoparticles were synthesized at $480^{\circ} \mathrm{C}$ and $25 \mathrm{MPa}$ for a range of $\mathrm{Na} / \mathrm{K}$ concentrations. It was found that in order to make the perovskite structure, the rearrangement of the $\mathrm{NbO}_{6}$ units in the presence of the alkaline ions was needed and this rearrangement, proceeded more rapidly with $\mathrm{Na}^{+}$ions. Therefore, formation of $\mathrm{K}_{0.5} \mathrm{Na}_{0.5} \mathrm{NbO}_{3}$ was achieved with an excess amount of $\mathrm{K}^{+}$ions..$^{\circ}$ Potential ferroelectric niobate materials were also made by the same authors (and evaluated for photocatalysts). ${ }^{441}$ Hayashi and colleagues also separately reported the synthesis of $\mathrm{KNbO}_{3}$ using a supercritical water flow system and investigated the materials for non-linear optics ${ }^{30,291}$ (see section 4.4).

In 2010, Aymonier and co-workers used spark plasma sintering for the densification of $15 \mathrm{~nm}(\mathrm{Ba}, \mathrm{Sr}) \mathrm{TiO}_{3}$ particles obtained a sc-water/alcohol flow type process. 439 This afforded reproducible and dense nanostructured ceramics that were made under low-temperature / high-pressure conditions that possessed novel properties. Because strain and ferroelectric polarization are intimately linked, it was observed therein that the final ferroelectric properties in the sintered disk could be significantly influenced by SPS process conditions. 439 Thereafter in 2016, Aymonier et al. made sub-20 nm barium titanate zirconate, $\mathrm{BTZ}\left(\mathrm{BaTi}_{1-\mathrm{y}} \mathrm{Zr}_{\mathrm{y}} \mathrm{O}_{3}\right.$ with $\left.\mathrm{o} \leq \mathrm{y} \leq 1\right)$ nanocrystals in flow (in a mixture of water and ethanol) over the whole solid solution range with a narrow size distribution ${ }^{426}$ The same researchers stated that ferroelectric materials were highly sensitive to changes in grain size when sintered (because strain and ferroelectric polarization have a direct connection). 439 Therefore, in order to retain the desired ferroelectric properties in a dense monolith, they again used spark plasma sintering to prevent significant grain growth during sintering. In comparison to the conventional sintering process, SPS enabled very high heating rates and short dwell times of few minutes to be used, leading to enhanced sintering kinetics. It was possible to obtain dense materials, largely keeping the initial grain size of the nanoparticles. Dielectric measurements were made on selected nanostructured ceramics (range $1 \mathrm{kHz}$ to $1 \mathrm{MHz}$, temperature range 70 to $450 \mathrm{~K}$ ). At ambient conditions, the permittivity values for these nano-scale ceramics were close to a value of 700 (instead of 3000 for micro-scale ones. Such a decrease in permittivity is fully consistent with a nanostructured ceramic (Figure 20). The researchers further commented on the quality of the disks by noting that superposition of the observed dielectric responses ( $1 \mathrm{kHz}$ to $1 \mathrm{MHz}$ ) in the paraelectric region, reflected the limited amount of extrinsic defects at the grain boundaries and highlighted the 
quality of their nano-structured ceramics, despite the high density of grain boundaries. ${ }^{426}$ Notably, both the BaTi ${ }_{1}$. $\mathrm{y}_{\mathrm{Z}} \mathrm{O}_{3}$ and the $(\mathrm{Ba}, \mathrm{Sr}) \mathrm{TiO}_{3}$ systems, were also the subject of in situ X-ray studies by the same researchers. 425,442

Figure 20

Multiferroic materials can possess magnetic and electric ordering parameters coupling in the same crystal structure. In particular, $\mathrm{BiFeO}_{3}$ is of interest as multiferroic material because both its electrical and magnetic ordering occur above room temperature; it shows antiferromagnetic ordering with a high Neel temperature of $\sim 643 \mathrm{~K}$ and ferroelectric ordering with a high Curie temperature of $\sim 1098$ k.437 For the Continuous hydrothermal synthesis of $\mathrm{BiFeO}_{3}$, Iversen et al. used stoichiometric amounts of iron(III) nitrate and bismuth nitrate in $0.4 \mathrm{M} \mathrm{HNO}_{3}$ (aq.) solution. ${ }^{443} \mathrm{~A}$ solution of $\mathrm{KOH}$ with concentration of $2 \mathrm{M}$ was used as the supercritical reagent and the synthesis was carried out at $400{ }^{\circ} \mathrm{C}$ (pressure 250 bar). In the case of the heterometallic oxide formation, it was suggested that it may have proceeded at high temperatures via a reaction between in situ formed crystalline bismuth oxide and amorphous ferric hydroxide. 443 In the case for the hydrothermal flow system, a small impurity of a $\mathrm{Bi}_{25} \mathrm{FeO}_{40}$ was detected in the sample, possibly due to the shortness of the reaction time in the continuous process. .43

\subsection{Magnetic Particles (Non Bio)}

Magnetic NPs (MNPs) have been proposed for a number of future technologies such as in high frequency miniature circuits super dense storage media, where the smaller size of the magnetic domains will allow the overall size of the devices to be reduced. Multiferroic materials (see earlier), with magnetic and electric ordering parameters coupling in the same structure, have attracted interest in data storage applications, spintronics, sensors, (quantum) electromagnets, and electronic devices. 409

Magnetic NPs (MNPs) have emerged as one of the important diagnostic and therapeutic materials for a variety of healthcare applications. When the dimension of these MNPs falls below a critical value (typically below $30 \mathrm{~nm}$ ), they exhibit superparamagnetism. $36,327,330,444$ Due to their comparable size with cells, these superparamagnetic particles find several biological applications (discussed elsewhere in this review). MNPs have been produced by CHFS since the technique was first developed. 67, 135, 323-327 In 1992, Adschiri and co-workers published a paper which described the flow synthesis of, amongst other things, $\mathrm{Fe}_{2} \mathrm{O}_{3}, \mathrm{Fe}_{3} \mathrm{O}_{4}, \mathrm{CO}_{3} \mathrm{O}_{4}$ and $\mathrm{NiO} .{ }^{72}$ This paper demonstrated that magnetic 
NPs could be produced using this technique, although not necessarily at a size likely to be useful (ranging from 50 $\mathrm{nm}$ for $\mathrm{Fe}_{2} \mathrm{O}_{3}$ to $200 \mathrm{~nm}$ for $\mathrm{NiO}$ ). Work on these homometallic oxides has continued, with other groups producing them, often as amongst their first papers on this subject, due to the simplicity of the materials. ${ }^{88,135,142,445-446}$ In particular, sub-10 nm particles of $\mathrm{Fe}_{2} \mathrm{O}_{3}$ and $\mathrm{NiO}$ and sub-20 nm particles of $\mathrm{CO}_{3} \mathrm{O}_{4}{ }^{136}$ have now been produced. Since there has been a lot of work on these materials, the effect of various conditions on their particle size is well known.

For very small $\mathrm{NiO}$ nanoparticles $(<10 \mathrm{~nm})$ that were made using a continuous hydrothermal flow pilot plant, the authors were able to unambiguously show the existence of a magnetically disordered outer layer (suggesting a "core / shell" model) in single crystal magnetic NiO using a combination of neutron powder diffraction and magnetometry measurements. ${ }^{138}$ Using neutron diffraction, allowed the magnetism of the $\mathrm{NiO}$ nanoparticles to be probed simultaneously with the structural information (the latter can also be obtained by conventional X-ray diffraction). By using uncoated antiferromagnetic $\mathrm{NiO}$ nanoparticles, the authors were able to completely separate the magnetic from the structural diffraction peaks; it was determined the nanoparticles, thus, consisted of ca. $5.2 \mathrm{~nm}$ diameter ordered antiferromagnetic core surrounded by a ca. $0.7 \mathrm{~nm}$ thick disordered shell. ${ }^{138}$ Further magnetic measurements showed that this disordered shell possessed significant polarisable magnetisation, (ca. $1 / 5^{\text {th }}$ that of pure $\mathrm{Ni}){ }^{138}$

Hao and Teja found that the particle size of $\mathrm{Fe}_{2} \mathrm{O}_{3}$ made in hydrothermal flow could be reduced from ca. 30 to 13 $\mathrm{nm}$ by reducing the initial $\mathrm{Fe}\left(\mathrm{NO}_{3}\right)_{3}$ concentration from $0.5 \mathrm{M}$ to $0.01 \mathrm{M}$. Other factors were not so important for $\mathrm{Fe}_{3} \mathrm{O}_{4}$ particle size control; neither temperature ${ }^{142}$ nor pressure ${ }^{88}$ had any big effect. Cote et al. experimented with reactor design, mixing streams of iron nitrate, sodium hydroxide and superheated water in different ways. ${ }^{142}$ They found that mixing the superheated water with the sodium hydroxide, then with the iron nitrate, gave more uniform (but not smaller) particles. This was also the case for $\mathrm{Co}_{3} \mathrm{O}_{4} ;$ the $\mathrm{UCL}$ authors have found that the use of $\mathrm{KOH}$ and $\mathrm{H}_{2} \mathrm{O}_{2}$ at $723 \mathrm{~K}$, can reduce the particle size of $\mathrm{CO}_{3} \mathrm{O}_{4}$ and $\mathrm{NiO}$ to 16 and $3 \mathrm{~nm}$, respectively. ${ }^{136}$

In more recent work, surface functionalised magnetite of ca. $4 \mathrm{~nm}$ has been reported in a single step process. ${ }^{323}$ At high citrate to iron values, some hematite was formed, however, upon reducing the citrate levels, only phase pure magnetite was obtained. ${ }^{323}$ The aqueous aggregates of dispersions were stable for more than 6 months at physiological pH. ${ }^{323}$ The NPs exhibited a superparamagnetic blocking temperature of $50 \mathrm{~K}$ at 500 Oe. Moreover, the peak in $M(T)$ was broad, indicating a gradual transition to the superparamagnetic state. The superparamagnetic state at ambient temperature was confirmed by hysteresis loops. The saturation magnetization was $37 \mathrm{emu}^{-1} \mathrm{~g}^{-1}$ at $10 \mathrm{~K}$, 
which was lower than the theoretical value of the bulk magnetite $\left(92 \mathrm{emu} \cdot \mathrm{g}^{-1}\right)$ and attributed to the surface spin canting, surface disorder and adsorbed species.

Using synchrotron source X-rays, in situ experiments were conducted for the one-step continuous hydrothermal synthesis of phase pure crystalline magnetite NPs. The fast heating of the apparatus, allowed very short reaction times under controlled and reproducible conditions, thus, chemical stabilizers were not required. Time-resolved in situ SAXS / WAXS experiments were carried out under supercritical conditions to study the growth of 6 nm superparamagnetic magnetite. The apparatus allowed rapid heating rates and pulsing of materials to study the evolution of the particles in situ; this allowed the team to better understand any transformations to more crystalline products in flow. 447 (see later in section 10.5 for overview of in situ research in flow)

More recently, there has been interest in the incorporation of well-ordered magnetic nanocrystals into magnetooptical (MO) devices for the spatial measurement of magnetic fields. 448 With the aim to achieving films displaying the so called magneto-optical Kerr effect (MOKE) [in which the light reflected from magnetized surfaces undergoes a change in both polarization and intensity on the basis of the strength and direction of magnetization], Adschiri and colleagues developed surface functionalised nano-magnetites via batch reactions (rather than flow). 448 They further developed routes to semi-continuous magnetic films, which hold promise for the development of high quality MOKE devices in the future. This could therefore, be an area in which flow reactors could make commercial surface functionalised nano-magnetites in future.

Another popular area of research for magnetic materials has been spinel structures containing $\mathrm{Fe}$, known as ferrites. They have a formula of $\mathrm{MFe}_{2} \mathrm{O}_{4}$. Cabañas et al. made the $\mathrm{Co}, \mathrm{Ni}, \mathrm{Zn}$ and $\mathrm{Co}_{x} \mathrm{Ni}_{(1-x)}$ oxide versions. ${ }^{135}$ They found that under the conditions used, the stoichiometry was rather variable and appeared to correlate with the extent to which the material would precipitate on its own in hydrothermal conditions. For example, Zn would not precipitate on its own at all and gave the lowest stoichiometry of ca. $\mathrm{Zn}_{0.7} \mathrm{Fe}_{2} \mathrm{O}_{4}$, whereas $\mathrm{Ni}$ had the highest yield on its own and in the ferrite, gave approximately the correct formula of $\mathrm{NiFe}_{2} \mathrm{O}_{4}$. The formula for $\mathrm{CoFe}_{2} \mathrm{O}_{4}$ was also close to stoichiometric. A later paper by some of the same authors studied the same material family using EXAFS and XANES 449 . In contrast to these reports, Cote et al. struggled to obtain the correct ratio of $\mathrm{Co}: \mathrm{Fe}$, and obtained a molar ratio varying from 1.74:1 to 1.87:1, with low levels of $\mathrm{Fe}_{2} \mathrm{O}_{3}$ as an impurity. ${ }^{450}$ They used metal nitrates as precursors (rather than acetates), and also $\mathrm{NaOH}$ as an additive, which may explain the differences in outcomes from others. The magnetic susceptibilities of the particles was reported as being similar to comparably sized $\mathrm{CoFe}_{2} \mathrm{O}_{4}$ parti- 
cles reported previously. Another group produced $\mathrm{CoFe}_{2} \mathrm{O}_{4}$ in a similar manner (using nitrates as precursors and $\mathrm{NaOH}$ as a pH modifier). ${ }^{297}$ They found that they had a slight excess of Fe in products, with Fe:Co ratios of ca. 2.04:1.00. The coercive field at $77 \mathrm{~K}$ was measured, and it was found to be 3730 Oe in the as-prepared powder, increasing to 7350 Oe after heat-treatment (at $873 \mathrm{~K}$ for $4 \mathrm{~h}$ ), which was a higher than the value for a spinel prepared by co-precipitation other work. ${ }^{451}$ Another spinel material $\mathrm{NiCO}_{2} \mathrm{O}_{4}$, was produced in flow at $723 \mathrm{~K}$ in flow with $\mathrm{KOH}$ and $\mathrm{H}_{2} \mathrm{O}_{2} \cdot{ }^{136}$ Aimable et al. also synthesised $\mathrm{Fe}_{3} \mathrm{O}_{41}, \mathrm{Fe}_{2} \mathrm{CoO}_{41}, \mathrm{Fe}_{2} \mathrm{NiO}_{41}$ and $\mathrm{Fe}_{2} \mathrm{ZnO}_{4} \cdot{ }^{156}$ of note in this report was a ca. $3 \mathrm{~nm}$ defective $\mathrm{Fe}_{2} \mathrm{CoO}_{4}$ spinel that was annealed at $600{ }^{\circ} \mathrm{C}$ to improve the structure, with a resultant particle size increasing to $34 \mathrm{~nm}$. However, the heat-treated material was still measured to possess a high coercive field of 7350 Oe at $-196^{\circ} \mathrm{C} . .^{156}$

Other reports on ferrites include the work of Hayashi and co-workers who used Fe( $\left(\mathrm{NO}_{3}\right)_{3}$ and $\mathrm{M}\left(\mathrm{NO}_{3}\right)_{2}[\mathrm{M}=\mathrm{Ni}$, $\mathrm{Cu}, \mathrm{Zn}$ ] aqueous solutions in a continuous hydrothermal reactor at $673 \mathrm{~K}(30 \mathrm{MPa}$ ) to make the corresponding particles $(<10 \mathrm{~nm})$ of $\mathrm{MFe}_{2} \mathrm{O}_{4}$ and $\gamma-\mathrm{Fe}_{2} \mathrm{O}_{3}$. The mixed phase, suggested the reactions did not go to completion in the time available..$^{42}$ It was shown that conversion of $\mathrm{Fe}^{3+}$ was more than 0.97 and was extremely fast, whereas that for $\mathrm{Ni}^{2+}, \mathrm{Cu}^{2+}$, and $\mathrm{Zn}^{2+}$, it showed some variation. ${ }^{452}$ Addition of $\mathrm{KOH}$ increased the conversion of the divalent cations to 0.87 , but there was always some phase separation using the conditions employed in that report. The particle size was observed to increase with increasing residence time. ${ }^{42}$ In a later report in 2011 , Sue et al. reported the synthesis of nickel ferrite from the metal nitrate salts, using a collision type micromixer (developed for rapid heating of a starting solution). 453 Temperature, residence time, and nitrate concentration were varied in the ranges 573 to $673 \mathrm{~K}, 0.02$ to $2.00 \mathrm{~s}$, and 0.05 to $0.50 \mathrm{~mol}^{\mathrm{kg}} \mathrm{kg}^{-1}$, respectively. Performing the reactions with no base, gave a Ni conversion $<2 \%$ (at temperatures up to $623 \mathrm{~K}$ ); this value reached $50 \%$ conversion of $\mathrm{Ni}$ (at $673 \mathrm{~K}$ ). It was suggested that suggest that in the flow process, under certain conditions, amorphous or low-crystallinity NPs nucleated as Ni-deficient $\mathrm{NiFe}_{2} \mathrm{O}_{4}$ at an early stage of the reaction and this is further crystallized through $\mathrm{Ni}^{2+}$ uptake. Furthermore, it was suggested that the aggregation growth process of nucleated nanoparticles was promoted under high-molality conditions and that these aggregates subsequently crystallized in flow (see Figure 21).453

Figure 21 
Amongst one of the most remarkable early magnetic nanomaterials produced via CHFS was barium hexaferrite, $\mathrm{BaO} \cdot 6 \mathrm{Fe}_{2} \mathrm{O}_{3}$. This material was first synthesized via CHFS in 1998 by Arai's group 77 who found that a rather complicated flow synthesis was required to produce the phase pure material. In the flow process, firstly the solutions of barium and iron nitrate were mixed with $\mathrm{KOH}_{\text {; }}$ this combined mixture was then mixed with superheated water at

$473 \mathrm{~K}$. Finally, the stream was mixed with even hotter water in the temperature range 723 to $773 \mathrm{~K} .{ }^{.7}$ In addition to this, a Ba:Fe ratio of $>0.5$ (i.e. more than a $6 x$ stoichiometric excess of barium) and an excess of $\mathrm{KOH}$, was used. The reason for mixing with superheated water twice was to produce an homogeneous reaction environment for the second mixing point, since it was calculated that $\mathrm{Ba}(\mathrm{OH})_{2}$ and $\mathrm{Fe}(\mathrm{OH})_{3}$ would be soluble in water at $473 \mathrm{~K}$. The $\mathrm{BaO} \cdot 6 \mathrm{Fe}_{2} \mathrm{O}_{3}$ particles were found to be in the size range ca. 100 to $200 \mathrm{~nm}$ and had a saturation magnetisation of 50 emu. $\mathrm{g}^{-1}$ and a coercively of 2550 Oe, which was similar to commercially available barium hexaferrite. ${ }^{77} \mathrm{~A}$ further publication featuring barium hexaferrite, managed to produce phase pure products at subcritical conditions (523 K). ${ }^{197}$ However, these particles had poorer magnetic properties than the report mentioned above (1622 Oe and 35.1 emu. $\mathrm{g}^{-1}$ ). This was rationalised by a lesser degree of dehydration at the lower synthesis temperature (and therefore higher TGA weight loss in the range 473 to 673 K). Subsequently, Kim et al. (from South Korea) conducted similar experiments to make barium hexaferrite and obtained similar results.454-455 More recently, Adschiri et al reported the synthesis of surface functionalised barium hexaferrite directly in a flow process. 456

\section{CATALYSIS APPLICATIONS}

Heterogeneous catalysts are important in the chemical industry and transport for a wide range of processes from the improvement of oil, gas and coal to polymerisation, in green chemistry, fine chemistry, environmental remediation, efficient conversion of biomass, or indeed to treatments of waste exhaust or industrial waste gases.7-9, 457 Advancing knowledge in the optimization or development of new catalysts is of enormous importance in order to increase efficiency and reduce energy costs. ${ }^{7-9,} 457$

Nanostructured catalysts have high specific surface area to volume ratios and high surface energies, which can lead to higher catalytic activity and potentially less side reactions (by allowing reactions at a lower temperature). ${ }^{9}$ There are a number of obvious areas where well-defined NPs made via CHFS or other methods, could be of benefit for catalysis applications. The CHFS method is particularly suited to preparing relatively unagglomerated catalyst particles with very high surface areas (e.g. typically nano titania with a diameter of $5 \mathrm{~nm}$, has a very high specific 
surface area of ca. $280 \mathrm{~m}^{2} \mathrm{~g}^{-1}$ ) and narrow particle size distributions, which would be ideal particle properties for some catalytic materials. The small size and narrow size distribution is possible to achieve in a flow system due to the rapid changes in supersaturation that occur and the relatively mild synthesis temperatures used (well under $1000^{\circ} \mathrm{C}$ ). This could be of advantage if one considers the improvements in efficiency that have been made for wellknown energy intensive heterogeneous catalyst reactions such as for the Haber process, which uses a nano-sized iron catalyst for converting $\mathrm{N}_{2}$ and $\mathrm{H}_{2}$ into $\mathrm{NH}_{3} \cdot{ }^{7}$ By varying the continuous reactor parameters or through the use of chemical additives, it is possible to affect particle shapes and sizes, e.g. obtain longer needles, ${ }^{120}$ etc., consequently, the CHFS method offers the potential to prepare catalysts with preferred surface orientations or particular edge / corner sites, which are catalytically relevant to the reaction in question. Such a capability, would help to make heterogeneous catalysts more active and selective (e.g. to minimise sites where unwanted side reactions may occur). Potential catalyst materials that can be prepared in CHFS systems, will be discussed below.

\subsection{Photocatalysts}

Photocatalysis using inorganic (semiconductor) photocatalysts is an emerging process for water and air purification and pollution remediation or for self-cleaning surfaces.4, 458-459 Fundamental and applied research on this subject has been performed all over the world during the last $40+$ years. 460

Titania and its Cation Doped Photocatalysts. The anatase form of titania is one of the best known photocatalysts as it is photostable, non-toxic, relatively inexpensive, low cost and usually active on its own or as a mixture.44 459, ${ }^{461-469}$ Anatase titania possesses a large band gap of $3.2 \mathrm{eV}$, consequently, by absorbing light with a wavelength < $400 \mathrm{~nm}$ (in the UV region), titania can generate electron hole pairs to drive a photocatalytic reaction. The anatase form of titania can generate electron-hole pairs to drive a photocatalytic reaction. In the report by Malinger et al. on anatase synthesis, the conditions of flow reactor were much slower than that used by the vast majority of researchers; they used a mixture of $\mathrm{TiCl}_{4}$ with $\mathrm{NaOH}$ in a tubular reactor, with residence times in the range 10 to 30 min and reaction temperatures in the range 120 to $220^{\circ} \mathrm{C} .{ }^{287}$ This did yield small particles in the size range 7 to 13 $\mathrm{nm}$, but should be seen more like a "continuous crystallizer" from an initial precipitate that is steadily heated in flow rather than a continuous hydrothermal process, where the water is heated first to create supersaturation and rapid reactions. 
The authors at UCL and others ${ }^{156}, 287,470$ have investigated many CHFS reactions of titania (anatase) and its doped materials, which can be made with a particle size of ca. $5 \mathrm{~nm}$ and surface area of almost $280 \mathrm{~m}^{2} \mathrm{~g}^{-1}$. In the CHFS work of the authors on $\mathrm{TiO}_{2},{ }^{260}$ it was found that the post-synthesis treatment conditions could greatly affect photocatalytic performance. The 'as prepared' powders were heat-treated in air in the temperature range 100 to $1000^{\circ} \mathrm{C}$ (at intervals of $100{ }^{\circ} \mathrm{C}$ )..$^{260}$ It was found that the anatase to rutile transition largely took place in the range 700 to $900{ }^{\circ} \mathrm{C}$ (although onset of rutile formation actually started at $500^{\circ} \mathrm{C}$ ). $\mathrm{TiO}_{2}$ (anatase) heat-treated at $300{ }^{\circ} \mathrm{C}$ in air, showed the best photoactivity for decolourisation of a simulated pollutant (methylene blue dye) under light irradiation for $4 \mathrm{~h}$. This was assumed to give the best balance of high surface area and good crystallinity. The photocatalytic activity of the sample heat-treated at $800{ }^{\circ} \mathrm{C}$, was also better than expected, which was attributed to formation of a mixture of anatase and rutile phases, which facilitated efficient electron transfer (to minimize electronhole recombination compared to anatase alone). Raman spectroscopy was used to monitor the peak changes with crystallite size in selected anatase materials; from the 'as prepared' $\mathrm{TiO}_{2}$ (peak at $\sim 150 \mathrm{~cm}^{-1}, 4.5 \mathrm{~nm}$ crystallite size as estimated by XRD) to heat-treated titania at $400{ }^{\circ} \mathrm{C}$ (peak at $\sim 147.7 \mathrm{~cm}^{-1} ; 17 \mathrm{~nm}$ crystallite size as estimated by $\mathrm{XRD}$ ). For samples heat-treated at $600{ }^{\circ} \mathrm{C}$ and above, the $\mathrm{E}_{\mathrm{g}}$ Raman band wavenumbers resembled those of bulk anantase (see figure 22 , peak at $\sim 144.5 \mathrm{~cm}^{-1}$ ). ${ }^{260}$

Figure 22

Iversen et al. made phase pure anatase $\mathrm{TiO}_{2}$ in the size range ca. 5 to $8 \mathrm{~nm}$ in supercritical isopropanol/water using a continuous hydrothermal reactor. 470 They investigated the catalysed photodegradation of rhodamine $B(R h B)$ under visible light irradiation and showed that the as-prepared $\mathrm{TiO}_{2}$ had a much higher efficiencies for photodegradation than a commercial photocatalyst (Degussa $\mathrm{P}_{25}$, which is in fact an optimised mixture of anatase and rutile). ${ }^{470}$ Anatase cannot normally absorb in the visible spectrum due to its large bandgap, however, in this case, as there was overlap between the energy distribution function of the excited $\mathrm{RhB}$ and the conduction band of $\mathrm{TiO}_{2}$, it was possible for the $\mathrm{TiO}_{2}$ to be photosensitized (by RhB dye). 470 The authors were also able to show a difference in the degradation process of $\mathrm{RhB}$ for the commercial photocatalyst and nano- $\mathrm{TiO}_{2} ;$ it was suggested that the less crystalline nano- $\mathrm{TiO}_{2}$, facilitated quicker RhB N-deethylation and slower cleavage of conjugated chromophore structure. In 
contrast, the more crystalline commercial photocatalyst, led to slower N-deethylation and quicker cleavage of conjugated chromophore structure.

CHFS made nano-titania was also recently reported as a feedstock for suspension plasma sprayed (SuPS) coatings; photocatalytic nanograined titania coatings with 90 to $100 \%$ anatase phase content, were obtained (with the remainder being rutile). ${ }^{37}$ This process of using a CHFS made suspension as a feedstock for a high temperature thermal spray process, can allow a small grain size to be retained in the coatings. This also removes the need for dry powder feeding in the spray apparatus.

Since ca. $97 \%$ of solar spectrum has wavelengths $>400 \mathrm{~nm}$, much of it cannot readily be accessed by pure undoped titania due to its large bandgap of $3.2 \mathrm{eV}$. Hence, there has been much interest in doping titania with other metals or ions in order to alter the bandgap and allow more of the solar spectrum to be absorbed and thus, potentially improve its photoactivity (see later). Conversely, introducing certain intra-band states due to doping, can also facilitate recombination or de-excitation pathways; therefore some materials can also have sunscreen applications (good absorbers but poor photocatalysts).

The authors and others have investigated libraries of doped CHFS made titanias as photocatalysts; for example, 54 crystalline doped nano-titanias (anatase), each with crystallite size of $<10 \mathrm{~nm}$ and possessing a large BET surface area (typically ca. $\left.285 \mathrm{~m}^{2} \mathrm{~g}^{-1}\right)$, were made using eighteen dopant elements at three different levels (0.5, 2.5 and 5 mol\%)..$^{304}$ It was found the $\mathrm{Ag}^{+}, \mathrm{Er}^{3+}, \mathrm{Gd}^{3+}, \mathrm{Pr}^{3+}, \mathrm{Sr}^{2+}, \mathrm{Zn}^{2+}$ and $\mathrm{Y}^{3+}$ materials were particularly good photocatalysts for dye decolourisation. In the case of nano-titania doped with either $\mathrm{Ce}^{3+}, \mathrm{CO}^{2+}, \mathrm{Fe}^{3+}, \mathrm{La}^{3+}, \mathrm{Mn}^{2+}, \mathrm{Nd}^{3+}, \mathrm{Ni}^{2+}$ or $\mathrm{V}^{4+}$, the photocatalytic activities were substantially reduced compared with the undoped material. ${ }^{304,471}$ Lester et al. also investigated $\mathrm{Co}$ and $\mathrm{Ni}$ doped titania photocatalysts; the nickel doped material showed a four-fold increase in photoactivity relative to the undoped material when tested using methylene blue photodegredation tests. 472

Photocatalytic tests are sometimes problematic because, they do not always give the same result when evaluating different model pollutants; therefore, it is often important to evaluate several different reagents to find catalysts that perform well. Therefore, some of the UCL authors evaluated several doped nano-titanias via a large-scale screening test including decolourisation of methylene blue (in the presence of oxygen), partial oxidation of a simple alcohol (propan-2-ol) and the degradation of aqueous solutions of dichloroacetic acid. ${ }^{262}$ A set of five dopants were used $\left(\mathrm{Zn}^{2+}, \mathrm{Sr}^{2+}, \mathrm{La}^{3+}, \mathrm{Ag}^{+}\right.$or $\left.\mathrm{Pr}^{3+}\right)$ at three different dopant levels (0.5, 2.5 or 5.0 mol\% of dopant) to give 15 unique doped titanias made using the CHFS system. The test results were cross-correlated and it was found that two of the 
tests, (DCA degradation and propan-2-ol), gave similar rank ordering for the nanopowders. Despite the popularity of methylene blue, the test results for it did not suggest a strong correlation with any other test studied.

Composites incorporating titania nanoparticles are also of interest for superior photocatalysts. 466,469 For example, films of titania containing hydroxyapatite (HA) nanoparticles coatings were prepared by taking HA made in an aqueous reaction at low temperatures in a plastic flow reactor and then using this as in a suspension with titanium isopropoxide for growing a HA/titania film via Aerosol Assisted Chemical Vapour Deposition (AACVD). ${ }^{359}$ It was shown that the presence of the HA nanoparticles, affected the morphology and crystallinity of the films. Selected materials containing HA showed superior photocatalytic activity and good photostability. 359

Anion Doped in Titania Photocatalysts. Anion doping in titania based photocatalysts can lead to improvements in the ability to absorb in the visible region. Visible-light activated titanium dioxide photocatalysts have been obtained by doping nitrogen, sulfur, or fluorine into the host lattice using a range of conventional synthesis methods. $462,464,467$ These anionic dopant species were better than transition-metal dopants with respect to stability of the catalyst. For example, for $\mathrm{N}$-doped titania $462,464,467$ a red shifting of the band-gap into the visible-range (owing to the mid-gap level of $\mathrm{N} 2 \mathrm{p}$ orbitals located between valence band and conduction band of titania), makes it possible to carry out a wide range of photocatalytic investigations under visible light. The range of doping concentrations reported recently for photocatalysts has been very narrow, generally lower than ca. 1 at $\%$. Some of the authors extended the aforementioned synthesis of 'as prepared' nano-titania powder made in CHFS by heat-treating the powder for $5 \mathrm{~h}$ in an $\mathrm{NH}_{3} / \mathrm{Ar}$ gas flow of $200 \mathrm{~mL}$. min'-1.473 Nitrogen-doped $\mathrm{TiO}_{2}$ (and titanium oxynitride) photocatalysts with only the anatase phase and a relatively small crystallite size (range 11.4 to $51.5 \mathrm{~nm}$; Figure 23a) were obtained under the nitridation temperatures in the range 400 to $700{ }^{\circ} \mathrm{C}$. Powders prepared at $600{ }^{\circ} \mathrm{C}$, had the best photocatalytic activity towards decolourisation of methylene blue under visible-light irradiation (Figure 23b), even better than the 'as precipitated' starting $\mathrm{TiO}_{2}$ nanopowder.

The higher activity of the N-doped samples, could be correlated with the modification in their absorption spectra, since the radiation used for the determination of the photocatalytic activity was in the edge of UV-Vis radiation. Under more severe conditions, it was possible to make polygonal shaped (and weakly agglomerated) TiN nanopowders using nitridation temperatures of $1000{ }^{\circ} \mathrm{C}$ with $5 \mathrm{~h}$ heating, under a gas flow at $200 \mathrm{~mL} \cdot \mathrm{min}^{-1} .473$ Figure $23 \mathrm{a}$ 
shows some of the changes in properties of the titania after heat-treatment in ammonia atmosphere) size, BET surface area and $\mathrm{N}$ level), whilst Figure 23b shows the photodegradation rates for methylene blue dye. 473

Figure 23.

Following the above report on $\mathrm{N}$-doping of titania, in 2010, $\mathrm{lhm}$ and co-workers reported the continuous one step hydrothermal synthesis of $\mathrm{N}$-doped titania and its photocataytic activity. ${ }^{474}$ The material was made directly in process from a precursor solution of titanium(IV)tetraisopropoxide (0.1 M) and nitric acid solution (0.8 M), which was left to stir for up to $3 \mathrm{~h}$ and then used at reaction temperatures of either 200,300 or $400{ }^{\circ} \mathrm{C} .474$ This yielded particle sizes of ca. 5, 10 and $16 \mathrm{~nm}$, respectively (by XRD analysis). Thus, the $\mathrm{N}$-doped titania was able to absorb not only UV but also visible light, which resulted in superior photocatalytic degradation of methyl orange (MO), when compared to standard photocatalyst Degussa p25.

Titanate Photocatalysts. Potassium hexatitanate is of interest as a photocatalyst and can be made via batch reactions under subcritical and supercritical water conditions; this work yielded fiber shaped photoactive particles that had a ruthenium oxide active catalytic phase on the surface. Such materials were shown to possess photocatalytic activity in water decomposition reactions. ${ }^{229}$ Despite being made from a batch hydrothermal synthesis, activity improvement of more than 13 -fold were obtained for the photocatalysts synthesized under (batch) subcritical water conditions and in the range 27 to 59 -fold for those synthesized under (batch) supercritical water conditions. Such materials would certainly be of interest in solar water splitting if made in flow reactors, where presumably the particle size should be smaller than batch.

Photocatalytically active potassium hexatitanate $\left(\mathrm{K}_{2} \mathrm{O} \cdot 6 \mathrm{TiO}_{2}, \mathrm{KTO}\right)$ can also be made in a continuous hydrothermal reactor. ${ }^{79}$ Starting materials used in this report were a titanium hydroxide $\left[\mathrm{Ti}(\mathrm{OH})_{4}\right]$ sol and potassium hydroxide $(\mathrm{KOH})$. It was observed that more $\mathrm{KTO}$ phase was formed when the $\mathrm{KOH}$ concentration was increased from 0.02 to $0.4 \mathrm{M}$, with a fixed concentration of $\mathrm{Ti}(\mathrm{OH})_{4}$ solution $(0.2 \mathrm{M})$. The products from these reactions ranged from being a mixture of spherical and fibrous particles $\left(T=350^{\circ} \mathrm{C}\right)$, to all fibrous $\left(T=420^{\circ} \mathrm{C}\right)$, depending on reaction temperatures used at the mixing point. ${ }^{79}$ The single-phase KTO particles obtained at a mixing point temperature of 400 ${ }^{\circ} \mathrm{C}$, were ca. $10 \mathrm{~nm}$ in width with lengths in the range ca. 500 to $1000 \mathrm{~nm}$. In contrast, particle sizes obtained at 420 
${ }^{\circ} \mathrm{C}$ were ca. five times larger than that obtained at $400{ }^{\circ} \mathrm{C}$. In particular, the researchers also evaluated the photocatalytic activity of KTO for photodecomposition of methanol. They observed that KTO obtained via CHFS, displayed ca. ten times greater activity than micron sized KTO particles produced in a conventional hydrothermal batch reactor (Figure 24).

Figure 24

The UCL authors used TiBALD, ${ }^{346}$ as a precursor for the synthesis of sodium titanate nano-sheets via CHFS 475 This solid was made using a solution of TiBALD that was pumped to meet a large excess of sodium hydroxide in flow, and thereafter, a superheated water feed at $450^{\circ} \mathrm{C}$ and 24.1 MPa. The UCL authors conducted photocatalytic testing on the freeze-dried nano-sheets (see Figure 25 for XRD data and TEM images), which showed outstanding performance in photo-decolourisation of methylene blue solution with visible-light irradiation, two times better than the commercial catalyst $\mathrm{P} 25 \cdot{ }^{346}$ More recently, Hayashi and colleagues reported the direct synthesis of sodium titanate (NTO) nano-sheets by a hydrothermal flow system from a Ti-sol and $\mathrm{NaOH}$ solution. Under supercritical conditions $\left(>400^{\circ} \mathrm{C}\right)$ at the initial $\mathrm{Na} / \mathrm{Ti}$ molar ratio $>5$, the product was microns in size and a few nm thick. It was also shown that the $\mathrm{Na}^{+}$ions could be removed from the solid to give a hydrated protonated titanate and that tetrapropylammonium (TPA) ions could also be used to intercalate into this Na free titanate. ${ }^{476}$

Figure 25

Zinc Oxide Photocatalysts. Zinc oxide ( $\mathrm{ZnO})$, a semiconductor that has a similar band gap to $\mathrm{TiO}_{2}$ and has been investigated for photocatalysis applications (as well as UV attenuating properties). The greatest advantage of ZnO is that it absorbs a large fraction of the solar spectrum and overall, more light quanta than undoped $\mathrm{TiO}_{2} 477$. Illumination of semiconductors such as $\mathrm{ZnO}$ and $\mathrm{TiO}_{2}$ with photons of energies greater than their band gap energies $(\mathrm{hv}<$ $400 \mathrm{~nm}$ ), promotes electron transitions from the valence band to the conduction band, leaving behind positive holes. 477 . The valance band potential is positive enough to generate hydroxyl radicals at the semiconductor surfaces and the conduction band is negative enough to reduce $\mathrm{O}_{2}$. The hydroxyl radical or hole $\left(h^{+}\right)$is a powerful oxidizing 
agent and attacks organic pollutants at or near the surface of semiconductor photocatalysts, resulting usually in their complete oxidation to $\mathrm{CO}_{2} 4,460$

It has been well known that the structural and morphological characteristics of $\mathrm{ZnO}$ determined by the different fabrication methods will influence its photocatalytic properties as well as degradation efficiencies towards organic molecules $^{12}$. In the earlier section, the UV attenuating properties of $\mathrm{ZnO}$ were discussed as well as its use in phosphors, ${ }^{172,} 204-205,207,209,478$ therefore, we will discuss its use in photocatalysis and methods for altering size, composition and shape in some detail.

The particle size and crystallinity of photocatalysts are amongst the most important factors in determining their photocatalytic properties ${ }^{11}$. Various methods adopted for the preparation of $\mathrm{ZnO}$ particles include the thermal deposition or co-precipitation ${ }^{479}$. Compared to such methods, CHFS syntheses have allowed the preparation of highly crystalline NPs with narrow size distribution and highly purity without further heat-treatment at higher temperatures ${ }^{197}$.

ZnO particles with high crystallinity were obtained by Ohara et al. using a CHFS process [see figure 26]. ${ }^{208}$ $\mathrm{Zn}\left(\mathrm{NO}_{3}\right)_{2}$ was pressurized to $30 \mathrm{MPa}$ at room temperature and heated to $390^{\circ} \mathrm{C}$ by mixing with supercritical water. Whiskers were produced when the concentration of metal salt solution was $<0.02 \mathrm{M}$. In the same work, room temperature photoluminescence spectra of $\mathrm{ZnO}$ whiskers was measured and showed a strong near-band-gap edge emission with broad red light emission, which indicated a possible use in UV-light emitting and laser devices.

Figure 26.

In order to reduce the particle size further, Sue and co-workers ${ }^{265}$ synthesized nano-sized ZnO fine particles (particle size range in this work was 15 to $25 \mathrm{~nm}$ ) in a CHFS system via rapid heating of an aqueous solution in a basic solution, using $\mathrm{Zn}\left(\mathrm{NO}_{3}\right)_{2}$ and potassium hydroxide solutions. The CHFS system pressure was maintained at $30 \mathrm{MPa}$, and temperatures used ranged from 573 to $673 \mathrm{~K}$. Particle size of $\mathrm{ZnO}$ from the CHFS system was about 10 times smaller than that of the reported analogous batch system reaction. They found that particle size of the product was increased with higher initial $\mathrm{Zn}\left(\mathrm{NO}_{3}\right)_{2}$ concentration. Also, by adding $\mathrm{KOH}$ into the system, smaller particles with higher product yields were obtained, due to the lower $\mathrm{ZnO}$ solubility in the $\mathrm{scH}_{2} \mathrm{O}$ mixture with base. ${ }^{265}$ The same group further examined the effect of $\mathrm{KOH}$ and $\mathrm{Zn}\left(\mathrm{NO}_{3}\right)_{2}$ (aq.) concentrations of starting solutions, flow rate, and 
other mixing factors on the particle size and morphology. ${ }^{89}$ Two methods of mixing were involved in this study. In the first method $(\mathrm{M} 1)$ supercritical water and $\mathrm{KOH}$ were first premixed and then mixed with $\mathrm{Zn}\left(\mathrm{NO}_{3}\right)_{2}$ in line, whilst in the second method $\left(\mathrm{M}_{2}\right)$, the supercritical water was premixed with $\mathrm{Zn}\left(\mathrm{NO}_{3}\right)_{2}$ first and then met with the $\mathrm{KOH}$ flow. The system temperature and pressure were maintained at $673 \mathrm{~K}$ and $30 \mathrm{MPa}$, respectively, and the products obtained were single phase $\mathrm{ZnO}$ (rhomboid shaped particles or rods under different conditions). It was reported in a previous publication on the flow synthesis of $\mathrm{ZnO}^{265}$, that increasing $\mathrm{KOH}$ concentration from $1 \times 10^{-3}$ to $2 \times 10^{-3}$ mol. $\mathrm{kg}^{-1}$, resulted in a decrease in particle size, however, no significant difference in particle size was observed when this was attempted for $\mathrm{ZnO}$ in this work. By changing the method of mixing from $\mathrm{M}_{1}$ to $\mathrm{M}_{2}$, a partial reduction in mean particle size, was observed (from 23 to $15 \mathrm{~nm}$ ) and the number of rods contained in the products, also increased. The authors proposed that the formation of rod-like $\mathrm{ZnO}$ was due to preferential precipitation and growth of crystals along the (0001) surface, resulting from the high supersaturation obtained via method M2. This work nicely demonstrated that the CHFS system could vary crystal morphology through control of nucleation and crystal growth in the heat-up, reaction, and cool-down cycle.

For the production of very small $\mathrm{ZnO}$, Sue et al. ${ }^{263}$ explored the effect of using mixing tee reactors with different inner diameters under fixed reaction conditions for a $\mathrm{CHFS}$ reaction involving $\mathrm{ZnSO}_{4}$ and $\mathrm{KOH}$ as precursors. In contrast to earlier work, ${ }_{1}^{89,265}$ they introduced cooling water at the exit of the mixing reactor to rapidly quench the products. Their results indicated that the introduction of cooling water minimized the product's particle size. Presumably, introduction of cold water to growing particles inhibited further crystal growth reactions of nuclei. Furthermore, in the same report, particle size was observed to decrease with decreasing inner diameter of the mixing tee reactor; this can be explained by the reduced heat-up time and enhanced mixing efficiency (of the salt and base solutions) for a small inner diameter mixer.

Viswanathan and Gupta reported the synthesis of ZnO NPs (as a potential photocatalyst material) via the oxidation of zinc acetate in supercritical water in a continuous tubular reactor. ${ }^{480}$ In their process, a preheated water and peroxide solution was pumped to meet a cold metal salt solution feed. Pressure and temperature of the system was kept at $400{ }^{\circ} \mathrm{C}$ and $245 \mathrm{~atm}$. The authors also stated that particles synthesized via this method were spheres in the range ca. 120 to $320 \mathrm{~nm}$, and that surprisingly, particle size did not appear to be greatly affected by flow rate or feed concentration. The wide particle size distribution and large particle size in flow, strongly suggests that the main 
mechanism for particle synthesis is via crystallization and non-uniform growth of smaller crystallites to form particles with a wide particle size distribution.

Sue and co-workers investigated both batch reactions and $\mathrm{CHFS}$ of $\mathrm{ZnO}$ using various zinc salts of $\mathrm{Zn}\left(\mathrm{CH}_{3} \mathrm{COO}\right)_{2}$ $\mathrm{ZnSO}_{41} \mathrm{Zn}\left(\mathrm{NO}_{3}\right)_{2}$, and base $(\mathrm{LiOH}, \mathrm{KOH})$ solutions. ${ }^{87}$ In one batch experiment, the diameter of particles synthesized in $\mathrm{LiOH}$ aqueous solution was smaller than that in $\mathrm{KOH}$ with otherwise identical conditions. It was suggested that particles from the $\mathrm{LiOH}$ reaction are in fact $\mathrm{Li}$-doped $\mathrm{ZnO}$, due to the high $\mathrm{Li}$-concentration found in the particles and closeness in the ionic radii allowing $\mathrm{Li}^{+}$(ionic radius $=1.28 \AA$ ) to partly substitute for $\mathrm{Zn}^{2+}$ (ionic radius $=1.22 \AA$ ) in lattice or even interstitial positions. In contrast, for the CHFS reaction product, phase pure and relatively Li-free $\mathrm{ZnO}$ was obtained with $\mathrm{Zn}\left(\mathrm{NO}_{3}\right)_{2}$ and $\mathrm{LiOH}$ as precursors. ${ }^{87}$ Particularly small particles (ca. $\left.16 \pm 8 \mathrm{~nm}\right)$, were obtained using a mixing nozzle with an inner diameter of $150 \mu \mathrm{m}$. This $\mathrm{ZnO}$ was ca. 35 times smaller than that synthesized in the corresponding batch apparatus. The particle diameter was also smaller than that obtained using $\mathrm{Zn}\left(\mathrm{NO}_{3}\right)_{2} / \mathrm{KOH}$ in a hydrothermal flow reaction under the same reaction parameters, which was reported in earlier work by the same group. ${ }^{265}$

Doped ZnO or Composite Photocatalysts. Viswanathan and co-workers ${ }^{41}$ further developed the concept of a

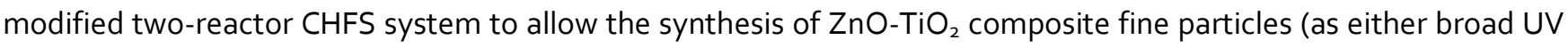
blocker materials or possibly as photocatalysts); two reactors were used in series and had two inlets each. Aqueous zinc acetate and hydrogen peroxide solution was fed through two inlets of the first reactor, and $\mathrm{TiCl}_{4}$ solution was fed through one of the feed lines in the second reactor, with the other feed line being the exit stream from the first reactor. The composite particles obtained were in the sandwich form, with $\mathrm{TiO}_{2}$ particles sandwiched between $\mathrm{ZnO}$ particles. Size range of $\mathrm{TiO}_{2}$ and $\mathrm{ZnO}$ were in the ranges 200 to $300 \mathrm{~nm}$ and 20 to $50 \mathrm{~nm}$, respectively. They also concluded that the formation of composite particles with $\mathrm{TiO}_{2}$ between $\mathrm{ZnO}$, could effectively minimize the agglomeration of $\mathrm{ZnO}$ particles.

UCL authors also used CHFS to synthesize crystalline NP catalysts in the Ti-Zn-O binary system. ${ }^{230}$ The as prepared nanopowders were heat-treated at $850^{\circ} \mathrm{C}$ for five hours in air to give photoactive semiconductor mixtures of rutile and zinc oxide and/or zinc titanates. These product powders were initially of interest for UV attenuators with low photoactivity (for their ability to scatter the sun's harmful UVA and UVB radiation). However, zinc titanate heterometallic phases or their phase mixtures, are also of interest as photocatalysts. Photocatalytic activity of the 
samples towards the decolourisation of methylene blue dye was also assessed over $6 \mathrm{~h}$. The reaction rate was calculated assuming first order kinetics (equation 5 below), which has been shown to fit well for photo-decolourisation of certain dyes including MB (in air). In all cases, a linear relationship was obtained for $\ln \left(C / C_{0}\right)$ against time

$$
\ln (\mathrm{C} / \mathrm{Co})=-k^{\prime} t
$$

$\left(C=\right.$ concentration at time $t, C_{0}=$ initial concentration, $t=$ time, $k^{\prime}$ is the composite rate constant $)$. Using XRD data for the as-prepared mixtures, it could be seen that the samples displaying low rates of decolourisation had broad indistinct XRD peaks, suggesting the samples were less crystalline. In contrast, the samples with more Zn, e.g. sample 7 (Ti: Zn nominal ratio 50:50) to sample 13 (Ti: Zn nominal ratio 0:100), the photocatalytic activity was amongst the highest of the as-prepared nanopowder mixtures. All the Zn-rich samples showed a sharp transition at the bandgap with a higher UVA absorption (see figure 9 earlier) for all the UV-Vis profiles of the as-prepared samples). ${ }^{230}$ It was suggested that the additional absorption could contribute to the higher activity, whilst surface oxygen deficiencies (seen in the XPS spectra of samples 8 to 13 that were high in Zn content) could be acting as capture centres for photoexcited electrons (to reduce recombination), enabling surface absorbed $\mathrm{OH}$ to form hydroxyl radicals that can take part in photocatalytic reactions. For the heat treated Ti-Zn-O binary samples, in most cases, a decrease in photocatalytic activity was observed in comparison to the corresponding as-prepared materials. The photoactivity also varied depending on the crystallinity and the absorbance as well as the structure and surface area. For the heat-treated powders, the $\mathrm{ZnO}$ and $\mathrm{Zn}_{2} \mathrm{TiO}_{4}$ mixtures displayed high activity, which may be of interest for UV photocatalysis applications. ${ }^{230}$ The highest activity was observed when the as-prepared sample containing a Ti:Zn ratio of ca. 10:90 that was heat-treated to form a phase mixture of $\mathrm{ZnO}$ and $\mathrm{Zn}_{2} \mathrm{TiO}_{4}$. The heat-treated sample (Ti: $\mathrm{Zn}$ nominal ratio 40:60) showed only one phase of pure zinc orthotitanate, which exhibited the lowest activity of all the samples made. ${ }^{230}$

More recently, a high throughput continuous hydrothermal $(\mathrm{HiTCH})$ flow synthesis approach was used to produce a library of 54-doped $\mathrm{ZnO}$ samples (18 different dopant ions at three concentration levels plus two undoped samples). Each sample was made from the reaction of an aqueous solution of basic zinc nitrate (and dopant ion or 
modifier) solution with a flow of superheated water at $450^{\circ} \mathrm{C}$ (pressure $24.1 \mathrm{MPa}$ ). The photocatalytic activity of the freeze-dried powders was evaluated (decolourization of methylene blue dye). It was observed that transition metal dopants tended to reduce photocatalytic activity because they formed levels deep in the band gap that acted as recombination centres. $\mathrm{Fe}, \mathrm{Ag}, \mathrm{Mn}, \mathrm{V}, \mathrm{Ni}$, and $\mathrm{Cr}$-doped samples all had weak colours and suppressed photocatalytic activity.

In general, increasing the doping concentration caused a lowering in the photocatalytic activity (silver and vanadium dopants were the exceptions to this, possibly due to a change in oxidation state of vanadium and possibly by a change in location of the silver ions. The authors suggest that $\mathrm{Fe}, \mathrm{Ag}, \mathrm{Mn}, \mathrm{Ni}$, and $\mathrm{Cr}$-doped $\mathrm{ZnO}$ may be suitable candidates for UV attenuation, because of their small $(<100 \mathrm{~nm}$ ) size, low photocatalytic activity and good UV absorption. ${ }^{270}$ Ideally, UV attenuators would have no visible absorption, but these samples had some limited colour, which may be acceptable in this application or in cosmetics. Co and Ti-doped zinc oxide had reasonable photocatalytic activity, comparable to that of the commercial $P_{25}$ photocatalyst. Furthermore, doping of rare earth ions generally increased photocatalytic activity. The order of activity for the decolourization of MB was $1.0 \mathrm{Nd}>1.0 \mathrm{Pr}>0.5 \mathrm{Y}>$ $0.5 \mathrm{Gd}>0.5 \mathrm{La}>1.0 \mathrm{Er}>\mathrm{ZnO} \gg 1.0 \mathrm{Ce}$ (where the number represents the dopant atomic percentage and with the most active dopant level being given per metal). Thus, for the rare earth dopants, it is likely that at higher concentrations, they acted as defect sites, which promoted recombination. Finally, it was found that the photodecolourisation activities of the rare-earth-doped $\mathrm{ZnO}$ were higher than that of undoped zinc oxide by up to a factor of two times and far exceeded that of the commercial standard photocatalyst $P_{25}$. Therefore, these materials may be promising candidates for photocatalytic applications. On the other hand, Ce-doping suppressed the activity of $\mathrm{ZnO}$, therefore, it may be a useful UV-attenuator material.

What was particualrly exciting in this work on doped $\mathrm{ZnO}$ samples was that the dopants of choice, gave failrly consistent changes in particle properties across the three loading levels used; these changes were see in particle size and BET surface areas (see figure 27), which not only shows the CHFS process to be highly reproducible, it also shows that the low level of dopants have a measurable effect on particle properties for $\mathrm{ZnO}$.

Figure 27 
Despite some interesting developments towards more active and more stable photocatalysts, ${ }^{4,16,482}$ real progress in this area has been slow, as comparison of results is difficult and it takes effort to build comprehensive structure-property-compositional relationships for these materials. To some extent there are now move towards standardization of photocatalytic reactions in order to be able to better compare different materials. ${ }^{483}$ One possible approach to discovering better photocatalysts and improving understanding of the science has been to make and screen many materials via high throughput synthesis and screening methods in order to cover large regions of compositional space in relatively short time. ${ }^{484}$ Such approaches have been recently applied by rapid synthesis in a high throughput CHFS system (HT-CHFS) and rapid screening methods. ${ }^{227}$ Furthermore, the ability to photocatalytically decolourize methylene blue or resazurin and other dyes in a simple parallel testing apparatus, was reported with either UV or a mixture of UV-visible light illumination. ${ }^{304}$ This work first suggested the potential of CHFS systems for the rapid manufacture of very large numbers of doped nanomaterials in relatively short times.

Bismuth-substituted ceria nanoparticles $\left(\mathrm{Bi}_{x} \mathrm{Ce}_{1-x} \mathrm{O}_{2-x / 2}\right)$ made in flow, have also been evaluated as photocatalysts for the photodecomposition of a model dye compound (rhodamine B). ${ }^{485}$ The compound was made in flow from a precursor made by dissolving $\mathrm{Bi}\left(\mathrm{NO}_{3}\right)_{3} \cdot{ }_{5} \mathrm{H}_{2} \mathrm{O}$ in a small amount of water by addition of nitric acid, then neutralizing with $\mathrm{NaOH}$. Then $\left(\mathrm{NH}_{4}\right)_{2} \mathrm{Ce}\left(\mathrm{NO}_{3}\right)_{6}$ was added followed by more neutralization, to give a $0.1 \mathrm{M}$ final solution concentration for the combined metal ions. 485 The reactor conditions were set at 350 and $375^{\circ} \mathrm{C}$, respectively, at 250 bar and a $15 \mathrm{~s}$ residence time was used. It was noted that as-prepared samples had some surface adsorbed $\mathrm{NO}_{x_{1}}$ water, and hydroxide groups; heat-treating the samples rendered them active for photocatalytic decomposition of rhodamine B. 485

\subsection{Heterogeneous Catalysts}

\subsubsection{Non-Precious Metal Catalysts / Supports}

This section will cover catalysts and catalyst supports containing mainly non-precious metals. As discussed earlier, where free NPs are used directly in a catalytic reaction, it is usually somewhat of an exception in heterogeneous catalysis. In the vast majority of cases, the metal particles (or other active species) are immobilized on a support, usually a metal oxide such as ceria, zirconia, alumina and silica, etc. 486

The support material of course possesses active oxygen species that are critically important in the reaction itself, e.g. for oxidation. The use of cation doping can have an effect of making some of this oxygen mobile under relative- 
ly mild conditions, which makes the catalyst more active (and may reduce the need for precious metals). Incorporation of a divalent cation onto a trivalent cation lattice (or a trivalent for a tetravalent) site, results in a charge imbalance that needs to be compensated by another lattice defect. This can usually happen in one of three ways,(i) creation of a host lattice oxygen vacancy, (ii) formation of a dopant (2+) interstitial ion or creation of (iii) a lattice selfinterstitial cation (i.e. the higher charge cation into the interstitial position). Because the CHFS process offers the ability to fine-tune dopant levels as well as synthesis conditions in a systematic way, it should be invaluable in understanding the interplay between such defects and their effects on performance.

In recent years, it has become apparent that the influence of the support on both the activity and the reusability of the catalyst is important. Therefore, much effort has gone into designing new catalyst supports. By manufacturing such supports as high surface area NPs (which may or may not be agglomerated), offers the possibility to then coat them with highly active metals in a subsequent sequence of impregnation and drying / firing steps.

Alumina Based Materials. $\mathrm{Al}_{2} \mathrm{O}_{3}$ is an industrially important catalyst support..$^{87}$ There are a number of reports on the continuous hydrothermal synthesis of the oxides of Al and its oxyhydroxide / hydroxides. An early paper attempting to make alumina via CHFS, was published by Arai's group, which described the production of $\mathrm{AlO}(\mathrm{OH}) .^{71-72,}$ 488 The same researchers and others, continued to work on this material and indeed eventually successfully prepared $\mathrm{Al}_{2} \mathrm{O}_{3}$ directly in a number of papers. ${ }^{489-490}$ For example, $\mathrm{Al}_{2} \mathrm{O}_{3}$ was produced directly at a high temperature of $773 \mathrm{~K}_{i} 490$ because researchers have accumulated so much data on the production of these materials, it has also become a fruitful system for theoretical/modelling studies 488,491

The Arai group papers from 1992, reported production of bohemite particles in the size range 0.1 to $1.5 \mu \mathrm{m}^{71-72}$ with considerable variations in particle size with temperature and pressure, and variation in particle shape with temperature and concentration of $\mathrm{Al}\left(\mathrm{NO}_{3}\right)_{3}$. As an indication of how the field of CHFS has progressed, by 2005, particle sizes in the range ca. $60-70 \mathrm{~nm}$ were made with smaller size distribution, and in this case, particle size and shape were found to be related to reaction temperature, pressure, reaction time, $\mathrm{pH}$ and concentration of reactants. ${ }^{81}$ More recently, highly anisotropic nano-crystalline boehmite was made in a flow reactor using near-critical and supercritical water and inexpensive $\mathrm{Al}\left(\mathrm{NO}_{3}\right)_{3} \cdot 9 \mathrm{H}_{2} \mathrm{O}$ as the precursor with a reaction time of $<2$ minutes. 492 The morphology of particles was found to be pressure dependent and at 400 bar pressure, bar-shaped nano-crystalline boehmite particles were obtained (Figure 28). At lower pressures, e.g. 200 bar, an inhomogeneous product result- 
ed. ${ }^{492}$ The size and shape of the average NP size was extracted from PXRD data and showed good agreement with TEM images. The dry boehmite particles under heat-treatment, maintained their overall morphology upon thermal transformation to $\gamma-\mathrm{Al}_{2} \mathrm{O}_{3}$, making this method an excellent route to developing alumina with small size. ${ }^{492}$

Figure 28

\section{Ceria Based Catalysts and Supports.}

Catalytic converters form part of a car's exhaust system and can remove harmful emissions, instead of release into the environment. 493 The catalytic converter is named so because it can covert $\mathrm{CO}$ and hydrocarbons into $\mathrm{CO}_{2}$ and water and $\mathrm{NO}_{x}$ into $\mathrm{N}_{2}$ and $\mathrm{O}_{2}$ (three way catalysts). A number of requirements are desirable in next generation autocatalysts including "fast light-off catalysts" that allow the catalytic converter to work at lower exhaust temperatures (better for short journeys), ${ }^{494}$ as well as more thermally durable catalysts 495 that can increase the life of the catalyst and allow the catalytic converter to be mounted closer to the engine. Furthermore, catalysts with reduced amounts (or more effective use of) precious metals that also display improved oxygen storage properties, are desirable. Cerium dioxide $\left(\mathrm{CeO}_{2}\right.$, ceria) and ceria based mixtures are becoming key constituents in catalytic systems as 2016 saw the 4oth anniversary since ceria was first used by Ford Motors (as an oxygen storage component in automotive catalytic converters). 496

Poliakoff and co-workers first reported the CHFS synthesis of very homogenous $\mathrm{Ce}_{x} \mathrm{Zr}_{1-\mathrm{x}} \mathrm{O}_{2}$ materials with highly defective pseudo-cubic phases, including some phases that could be stabilized beyond previously reported phase boundary limits. ${ }^{97}$ The nanomaterials were obtained with the surface areas as high as $180 \mathrm{~m}^{2} \mathrm{~g}^{-1}$. Korean researchers in collaboration with Hanwa Corporation of Korea, ${ }^{404}$ measured the oxygen storage capacity (OSC) of similar ceriazirconia nanomaterials and found that they had excellent OSC with very labile oxygen at low temperatures.

The importance of thermal durability in three-way catalyst (TWCs) has been highlighted earlier; ${ }^{495}$ some of the authors at UCL reported the CHFS syntheses of Ce-Zr-La-Y oxide solid solutions; the effects on OSC were studied for both the as-dried materials as well as those subjected to accelerated ageing. ${ }^{363}$ The samples were each dried using either freeze or hotplate drying and then either analyzed as dried powders or after an further $10 \mathrm{~h}$ heattreatment (at either 1000,1100 or $1200^{\circ} \mathrm{C}$ ). The TPR profiles were very different depending on the thermal history of the samples and can be summarized as follows; (1) despite the $10 \mathrm{~h}$ heat-treatments (BET surface areas typically < 
$\left.15 \mathrm{~m}^{2} \mathrm{~g}^{-1}\right)$, the samples generally possessed high oxygen storage capacities and (2) the freeze-dried materials displayed highly mobile oxygen at low temperatures. ${ }^{363}$

CHFS has tremendous potential in the synthesis of support or active three way catalysts for cars in systems such as Ce-Zr-Y oxide based supported catalysts. One of the major questions around the use of CHFS is if the materials adversely sinter with aging and what is the nature of the interaction when precious metals are added? As has been mentioned earlier, low temperature co-precipitation methods can suffer from the oxides precipitating out at very different rates leading to inhomogeneities in binary solid solutions. The CHFS method can allow rapid precipitation under precise conditions and hence, perhaps a different view of solid solution solubility in phase diagrams, may emerge.

In an extension of the work of some of the UCL authors and others in Ce-Zr oxide systems 75,497 as well as YSZ (yttia stabilized Zirconia) solid solutions or mixtures,357, 498 a new high throughput (HT) synthesis capability was developed to make an entire phase diagram (66 samples) of Ce-Zr-Y oxide in 12 h. $3{ }^{33-34}$ Consequently, the following questions were posed;

1) What is the phase behavior and solid solution solubility after heat-treatment at $1000{ }^{\circ} \mathrm{C}$ (typical temperature at which such materials are aged for testing) and how does it differ from conventional methods?

2) Does the process of synthesis allow the materials to be made with gradual changes in properties (such as unit cell volume changes) across the compositional map?

3) Are the phases metastable? and

4) Which materials compositions sinter the most?

From the 66 samples, the UCL authors obtained Raman data, metal analyses via EDS and high resolution XRD data during the commissioning experiments for a new high throughput automated beamline XRD (Station l11 at Diamond Synchrotron in Oxford) which allowed very high quality data sets in less than 10 mins per set.33 The Raman assignments were then used to guide the batch Reitveld refinements in order to suggest trends in lattice parameters with changes in composition. This work allowed the team to reveal unusually large phase fields of solid solution solubility, that were not known previously (Figure 29). In a follow-up publication, the pre-heat treated samples were also characterized. ${ }^{33}$ 
Figure 29 .

In Figure 29a, the compositional space for $\mathrm{Ce}_{x} \mathrm{Zr}_{y} \mathrm{Y}_{z} \mathrm{O}_{2-\delta}$ (heat-treated) is shown in which each hexagon represents a single sample, and the nominal molar content of $\mathrm{Ce}, \mathrm{Zr}$, and $\mathrm{Y}$ in each sample is proportional to the amount of red, green, and blue colour, respectively. Samples were synthesized in sequential order starting at number 1 and ending at 66 (see Figure 29a) thus, reduced the possibility of cross contamination for the binary compositions. In Figure 2gb, a simplified phase diagram for $\mathrm{Ce}_{x} \mathrm{Zr}_{y} \mathrm{Y}_{z} \mathrm{O}_{2-\delta}$ is shown that was made on the basis of synchrotron PXRD data. The figure shows the following phase types; fluorite (shown in gray), monoclinic $\mathrm{ZrO}_{2}$ (shown in yellow), tetragonal $\mathrm{ZrO}_{2}$ (shown in red), bixbyite (shown in blue), and a $2 \times 2 \times 2$ oxygen-deficient fluorite superstructure (pseudo- $\mathrm{Y}_{2} \mathrm{O}_{3}$ ) with oxygen-vacancy ordering (shown in green). Hexagons in a single colour indicated single-phase material, while the hexagons with three dots indicated two-phase behavior, and those with striped colours indicated a single-phase region, where there is a smooth transition between fluorite and the $2 \times 2 \times 2$ oxygen deficient fluorite superstructure. In Figure 29c, the lattice parameter information from Rietveld-fitted PXRD data was converted to mean volume per metal oxide $\left(\mathrm{MO}_{2-\delta}\right)$ unit and shows a smooth pseudo-linear variation with mole fraction. Figure 29d shows the relative crystallite size (as indicated by the circle diameters) obtained by application of the Scherer equation to the strongest PXRD peak (at $2 \theta \approx 15^{\circ}$ ) for each of the 66 samples (taking instrumental peak broadening into account). The largest crystallite size was for pure $\mathrm{CeO}_{2}$ (shown in the diagram as a filled hexagon).

More recently, the authors at Zhejiang published an article describing the preparation of what are sometimes known third generation TWCs, which introduced alumina doping and / or $\mathrm{\gamma}-\mathrm{Al}_{2} \mathrm{O}_{3}$ particles to prevent sintering of the $\mathrm{Ce}_{x} \mathrm{Zr}_{1-x} \mathrm{O}_{2}$ grains ${ }^{92}$ (see later in section on precious metals supported catalysts). In a further report, some of the authors investigated the possible correlations between measured properties and spectroscopic or other data. ${ }^{471}$ This is because one of the wholly grails for the development of TWCs (and other catalysts) is to discover compositions or conditions of active catalysts, without the need to actually measure each and every sample (OSC and TPR profiles can take an order of magnitude longer than the time required to make materials). Ideally, if inferential measurements can be used, such as correlation of spectral peaks to desired properties, this would certainly aid such optimizations. Chemometrics were applied to the spectroscopic and other data to investigate any significant correlations 
between the measured surface areas, OSC and between peaks in the spectroscopic data (e.g. Raman data)..$^{411}$ No doubt, a much larger dataset will benefit such investigations in the future.

Highly active ceria-zirconia oxide / graphene nanocomposites made via CHFS, were used as a catalyst for the direct synthesis of DMC (dimethyl carbonate) from $\mathrm{CO}_{2}$ and methanol. DMC has low toxicity and high biodegradability, which makes it a green reagent for the production of polycarbonates. The effect of various catalyst test parameters such as the reaction time, temperature, $\mathrm{CO}_{2}$ pressure, catalyst loading and the use of a dehydrating agent, was evaluated. The ceria-zirconia/graphene catalyst was easily recycled and reused several times without any significant deterioration in its catalytic performance. ${ }^{362}$

$\mathrm{ZrO}_{2}$ based catalyst supports (some of which contain ceria) have also been made via CHFS and their catalytic properties evaluated. Galkin et al. produced a range of doped or pure zirconiass ${ }^{369}$ including $\mathrm{Ce}_{0.5} \mathrm{Zr}_{0.5} \mathrm{O}_{2}$, $\mathrm{Ce}_{0.1} \mathrm{Y}_{\times} \mathrm{Zr}_{0.9} \mathrm{O}_{2}, \mathrm{Zr}_{1-\mathrm{x}} \mathrm{Y}_{\times} \mathrm{O}_{2}, \mathrm{Zr}_{1-\mathrm{x}} \mathrm{In}_{\times} \mathrm{O}_{2}$, and $\mathrm{ZrO}_{2}$ (as well as $\mathrm{Pd} / \mathrm{Rh} / \mathrm{ZrO}_{2}$ ).

Other applications for nano-sized ceria based catalysts are for oxidation reactions; Kim et al. impregnated various transition metals (e.g. $\mathrm{Mn}, \mathrm{Fe}, \mathrm{Co}, \mathrm{Ni}$, and $\mathrm{Cu}$ ) onto a $\mathrm{CHFS}$-made $\mathrm{CeO}_{2}$ support for the catalytic wet oxidation (CWO) of phenol. The catalysts showed excellent activity and $\mathrm{CO}_{2}$ selectivity. 499 In other oxidation catalysis research using ceria containing materials, some of the authors attempted to produce a series of transition metal codoped ( $\mathrm{Mn}$, $\mathrm{Cr}$ or $\mathrm{Cu}) \mathrm{Ce}_{x} \mathrm{TiO}_{2}$ samples and revealed that the $\mathrm{Cu}$ and $\mathrm{Ce}$ co-doped samples showed the highest activity in the catalytic oxidation of toluene, which was comparable to some noble metal catalysts. ${ }^{278}$ The authors further reported that a Strong-Metal Support Interaction (SMSI) feature was also obtained in CHFS made a $\mathrm{Ce}_{x} \mathrm{TiO}_{2}$ sample which showed superior activity in Selective Catalytic Reduction (SCR) of $\mathrm{NO}$ with $\mathrm{NH}_{3} .{ }^{500}$ The special contact between $\mathrm{CeO}_{2}$ and $\mathrm{TiO}_{2}$ had induced enriched surface oxygen vacancies in the sample, which effectively facilitated the oxidation of $\mathrm{NO}$ to $\mathrm{NO}_{2}$ in $\mathrm{SCR}$ reactions. These contacts also lowered the $\mathrm{NH}_{3}$ oxidation over $\mathrm{CeO}_{2}$, effectively retaining the $\mathrm{NH}_{3}$ for $\mathrm{SCR}$ reactions. ${ }^{500}$

Ceria-zirconia based solid solutions incorporating other elements will be important materials in future automotive catalysts and would benefit from applying a materials discovery approach if new and rapid testing methods ${ }^{501}$ or diagnostic analytical methods can be developed.

Perovskites or Related Structures. Often, in order to obtain the desired balance of properties in an inorganic support, heterometallic supports are used rather than homometallic ones. In general, materials with exceptional 
framework structures (e.g. perovskites) are accompanied with extraordinary properties that are of interest for many catalyst applications. These materials, however, tend to be difficult to make, requiring multi-step and prolonged processes to obtain pure phases. ${ }^{02}$ The authors believe that the $\mathrm{sc}-\mathrm{H}_{2} \mathrm{O}$ crystallizing environment could be an ideal reaction medium to solve this problem, as it will be able to facilitate either direct synthesis (due to extreme conditions) or provide mixtures that can readily react to form complex structures. The former could be a new focus for CHFS. For example, $\mathrm{Ca}_{0.8} \mathrm{Sr}_{0.2} \mathrm{Ti}_{0.9} \mathrm{Fe}_{0.1} \mathrm{O}_{3-\delta}$ was synthesized directly via CHFS; the material was of interest as a support for nickel catalysts in the partial oxidation of methane. ${ }^{431}$ These type of doped perovskite materials usually require a high temperature synthesis in order to be made phase pure (in excess of $850{ }^{\circ} \mathrm{C}^{362}$ ), which usually results in low surface areas (typically ca. $5 \mathrm{~m}^{2} \mathrm{~g}^{-1}$ or less). A continuous hydrothermal process was used to produce this phase pure material at a temperature of only $673 \mathrm{~K}$, resulting in a nanopowder with a BET surface area of $70.6 \mathrm{~m}^{2} \mathrm{~g}^{-1}$. Other notable flow synthesis of perovskites structures include $\mathrm{Pr}$-doped $\mathrm{CaTiO}_{3}$, which is discussed in the section on nanophoshors (section 4.1.). ${ }^{216}$

Researchers working at the University of Nottingham (UK) reported the synthesis of phase pure catalytically active and relatively high surface area $\left(19 \mathrm{~m}^{2} \mathrm{~g}^{-1}\right)$ perovskite $\mathrm{La}_{2} \mathrm{CuO}_{4}$ using $\mathrm{CHFS}$ synthesis followed by a further heattreatment. ${ }^{140}$ TPR of the material was used to assess the behavior of the material compared to "traditionally prepared" low surface area $\left(0.4 \mathrm{~m}^{2} \mathrm{~g}^{-1}\right) \mathrm{La}_{2} \mathrm{CuO}_{4} \cdot{ }^{140}$ The TPR trace showed that the CHFS (plus heat-treatment) material displayed a low temperature first reduction peak assigned to the loss of weakly bound oxygen (this was ca. $100^{\circ} \mathrm{C}$ lower than the first reduction peak for "traditionally prepared" material). This indicated profoundly increased bulk oxygen mobility for the CHFS-made sample (even though the total hydrogen uptake was virtually identical for both types of samples). The oxidation of $\mathrm{CO}$ (using $\mathrm{CO}$ pulsing experiments) was also compared and the CHFS sample had ca. 2.5 times the oxidation activity compared to the "traditionally prepared" $\mathrm{La}_{2} \mathrm{CuO}_{4} \cdot{ }^{140}$

The authors at Zhejiang University recently studied the potential catalytic applications of $\mathrm{La}_{n+1} \mathrm{Nin}_{n} \mathrm{O}_{3^{n+1}}$ compounds ( $\mathrm{n}$-layered perovskite block separated by a rocksalt LaO layer) that were prepared by using CHFS followed by high temperature heat-treatments. ${ }^{503}$ These $\mathrm{La}_{n+1} \mathrm{Ni}_{n} \mathrm{O}_{3 n+1}$ materials were evaluated for the catalytic oxidation of toluene and methane with the composition and lability of active oxygen species being investigated by analytical techniques including, XPS $\mathrm{O}_{2}$-TPD and $\mathrm{H}_{2}$-TPR. They found that the $\mathrm{LaNiO}_{3}(n=\infty)$ material had the greatest amount of chemisorbed oxygen and the lowest activation energy $\left(E_{a}, c a .70 .1 \mathrm{~kJ} \mathrm{~mol}^{-1}\right)$ for tolvene oxidation. The $\mathrm{La}_{4} \mathrm{Ni}_{3} \mathrm{O}_{10}(\mathrm{n}=3)$ compound showed a superior lattice oxygen mobility and the lowest $\mathrm{E}_{\mathrm{a}}\left(\mathrm{ca}\right.$. $\left.111.0 \mathrm{~kJ} \mathrm{~mol}^{-1}\right)$ in me- 
thane oxidation (see Figure 30). The interstitial oxygen ions in the LaO layers were not active in both oxidation processes. ${ }^{503}$

Figure 30

Ultrafine perovskites (at ca. $5 \mathrm{~nm}$ ) were also prepared by using CHFS route. Islam et al. ${ }^{504}$ recently reported that with careful control of the NP residence time in CHFS reactor; ultrafine $\mathrm{La}_{1-x} \mathrm{Sr}_{x} \mathrm{MnO}_{3}$ ( $\mathrm{x}$ in the range 0.1 to 0.3 ) nanocrystallites could be directly produced with no need for an additional heat-treatment. ${ }^{504}$ They indicated that the direct production of perovskites should ideally be conducted under supercritical water conditions $\left(\mathrm{T}>374^{\circ} \mathrm{C}\right.$ and pressure higher than $22.1 \mathrm{MPa}$ ). Only the residence time at ca. $0.17 \mathrm{~s}$ was required to yield the phase pure $\mathrm{La}_{1-x} \mathrm{Sr}_{x} \mathrm{MnO}_{3}$ ( $x$ in the range 0.1 to 0.3 ) product..$^{504}$ Some of the authors recently revisited this direct synthetic approach using $\mathrm{H}_{2} \mathrm{O}_{2} .{ }^{505}$ Ultrafine $\mathrm{LaMnO}_{3}$ nano-crystallites could be produced as long as the residence time was $>$ ca. $0.7 \mathrm{~s}$. After being subjected to $\mathrm{H}_{2}$-TPR and $\mathrm{O}_{2}$-TPD, the material yielded distinct $\mathrm{H}_{2}$ consumption and oxygen desorption peaks. Compared to "traditionally prepared" $\mathrm{LaMnO}_{3}$ material, the CHFS made sample had a higher surface area and greater oxygen mobility (due to the creation of anion vacancies from the doping of $\mathrm{Sr}^{2+}$ for the $\mathrm{La}^{3+}$ cation), which was comparable to some noble metal containing catalysts (low $E_{a}$ for toluene oxidation ca. $54 \cdot 3 \mathrm{~kJ} \mathrm{~mol}^{-1}$ ).505

In a further report using $\mathrm{CHFS}$, some of the authors co-precipitated a high surface area $\mathrm{LaCoO}_{3} / \mathrm{MgO}$ composite mixture from a solution of the metal salts..$^{506-507}$ The as-prepared product was then heat-treated in air at $700{ }^{\circ} \mathrm{C}$ for 2 h. The aim was to obtain a high dispersion of active $\mathrm{LaCoO}_{3}$ with the $\mathrm{MgO}$ support (the latter acting as a sintering retardant) as well partial doping of $\mathrm{Mg}^{2+}$ into the perovskite to increase oxygen vacancies. ${ }^{506-507}$ The latter promoted the formation of active oxygen species, resulting in remarkable tolvene or methane oxidation performances (such $\mathrm{Mg}$-doped $\mathrm{LaCoO}_{3}$ materials are also potential of interest as visible light photocatalysts). ${ }^{08}$ Further modification onto the $\mathrm{A}$ site in $\mathrm{LaCoO}_{3}$ perovskite was also undertaken, where $\mathrm{Ca}^{2+}$ was introduced to partially replace $\mathrm{La}^{3+}$ ions. ${ }^{507}$ Dual-site substitution of $\mathrm{Ca}^{2+}$ and $\mathrm{Mg}^{2+}$ was found to be beneficial for toluene oxidation (with an apparent lowering of activation energy to only $34 \mathrm{~kJ} \mathrm{~mol}^{-1}$ that was on par to some catalyst incorporating precious metals). Clearly, the introduction of $2+$ cations into the structure, had created anion vacancies (charge compensation) and consequently, highly active oxygen anions. However, this substitution had somehow deactivated the methane oxi- 
dation as the substituted $\mathrm{Ca}^{2+}$ could inhibit the generation of surficial lattice oxygen and decreased the reducibility of $\mathrm{LaCoO}_{3}$.

Petrochemical/Coal Related Catalysts. The Fischer-Tropsch (FT) process involves chemical reactions that convert a mixture of carbon monoxide and $\mathrm{H}_{2}$ (syngas) into liquid hydrocarbons. ${ }^{509}$ A number of transition metal catalysts based on Fe or Co etc. can be used for such processes. Cobalt catalysts are more active for Fischer-Tropsch (FT) synthesis when the feedstock is natural gas and therefore, there has been interest in making oxides of cobalt that can be reduced in situ to cobalt metal on a silica support. ${ }^{509}$ As such catalysts are used for FT-reactions, eventually the catalyst has to be regenerated (via oxidation) to remove / burn off any carbonaceous deposits. It has been found that solid Co metal particles $>10 \mathrm{~nm}$ can form hollow particles on oxidation that fracture after regeneration (i.e. when reducing back to the active Co metal); this is cycle is often referred to as Reduction-Oxidation-Reduction (ROR) treatment..$^{510}$ Particles of cobalt metal $<11 \mathrm{~nm}$ appear to be relatively stable to multiple oxidation and reduction cycles so the catalysts can often be regenerated without loss of the active particle integrity. ${ }^{510}$ Thus, the authors investigated routes to control the size of cobalt oxide particles to below $10 \mathrm{~nm}$ (ideally) and study size control under different growth conditions. ${ }^{171}$

The authors used an arrangement of two confined jet mixers in series with each other in, which the first mixer formed the spinel nanoparticles and the second one, acted as a cooling quench (with only ambient temperature water). The concept was to study the nucleation and growth behaviors in the first mixer under laminar and turbulent conditions at various $\mathrm{pH}$ regimes and try to use this to explain the yields and size distributions obtained at different mass production rates. Four different tandem mixer configurations in either laminar or turbulent flow regimes, were studied in the pipe downstream of the mixer. ${ }^{171}$ The distribution of sizes of the NPs was influenced by the flow regime, the concentration of the precursor, and the presence of the quench. Mechanisms of complete nucleation followed by growth via coalescence and incomplete nucleation followed by growth from solution, were observed. The nature of the nucleation and growth were strongly dependent on the mixing conditions as well as the initial concentration of cobalt acetate and resulting product $\mathrm{pH} .{ }^{171}$

Under certain conditions, the quench feed allowed a reduction of the average crystallite size without significantly influencing the yield of the reaction. An increase of the crystallite size with increasing concentration, was observed under turbulent mixing conditions, whereas a decrease in size with increasing concentration, was observed for lami- 
nar mixing conditions. Overall, the main benefits of the CHFS synthesis method in this case was the ability to make NPs with a D50 size of $6.5 \mathrm{~nm}$ at a rate of $20 \mathrm{~g} \mathrm{~h}^{-1}$ using a laboratory scale continuous reactor. ${ }^{171}$ No doubt, further exploration of these NPs for suitability for FT-catalysis, will be investigated in the future to understand the effects of activity and selectivity with particle size and faceting.

Whist FT reactions make hydrocarbons from small molecules, certain high surface area catalysts can also be used to break C-C bonds. Matson et al. produced a number of iron-based materials to test for activity in C-C bond scission reactions in napthylbibenzylmethane (as a model reaction for the liquefaction of coal). ${ }^{134,}{ }^{511}$ They compared the reactions of these materials to similar materials produced by a modified reverse micelle reaction, and to conventionally produced iron compounds. In all, the researchers identified five different phases produced by CHFS, although they suggested that some of these phases were not phase pure. They found that lower temperature CHFS reactions (T-mixing point at $\sim 200{ }^{\circ} \mathrm{C}$ ) tended to favor the formation of ferrihydrites $\left(\mathrm{Fe}_{5} \mathrm{HO}_{8} \cdot{ }_{4} \mathrm{H}_{2} \mathrm{O}\right.$ ), whereas at higher temperatures ( $\mathrm{T}$ mixing point at $\sim 300{ }^{\circ} \mathrm{C}$ ), crystalline hematite was formed. ${ }^{134}$ At low temperatures, the shortest residence times produced the most disordered ferrihydrite phase 2 -line ferrihydrite (meaning it has only two broad lines in its powder XRD pattern). This tended towards formation of the more ordered 6-line ferrihydrite (six lines present in the powder XRD pattern) with longer residence times. Use of acetate buffer or urea appeared to encourage the formation of ferrihydrites over hematite. ${ }^{134} \mathrm{Fe}(\mathrm{II})$ was rapidly oxidized to $\mathrm{Fe}(\mathrm{III})$ under hydrothermal conditions to form hematite, except when urea was used (resulting in magnetite being formed). Finally, iron oxyhydroxysulphate formed by adding $\mathrm{Na}_{2} \mathrm{SO}_{4}$ to the reagent feed.

On testing these iron-based catalysts, they found that the 6-line ferrihydrite, magnetite and iron oxyhydroxysulphate had amongst the highest performances of the CHFS-produced materials, each with over 90\% yield and selectivity. ${ }^{134}$ The CHFS-made catalysts were better than those made via reverse micelle or more conventional synthesis materials, although the authors were not able to conclude whether this was specifically due to particle attributes or the constituent phases. This was because of the difficulty in directly comparing the products from each synthesis method (different phases, particle sizes, surface areas etc.)

Bismuth-doped ceria catalysts are used as "oxygen reservoirs" in the oxidative dehydrogenation of propane. In such reactions, the lattice oxygen is used to selectively combust hydrogen from the dehydrogenation mixture at ca. $550^{\circ} \mathrm{C}$. Similar materials are also of interest as photocatalysts. More information on the flow synthesis of bismuthsubstituted ceria nanoparticles can be found in the section on photocatalysis (Section 7.1). ${ }^{485}$ 
Despite, their extensive use in the petrochemical industry as catalysts, to date, there has only been one report of the production of zeolites via a continuous hydrothermal process. Zeolites are microporous aluminosilicate materials onto which, molecules may be adsorbed to catalyze chemical reactions or for environmental decontamination (in the latter via cation exchange reactions). Furthermore, proton-exchanged $(\mathrm{H})$ zeolites are extensively employed in for cracking crude oil fractions into fuels and chemical feedstocks for other processes. ${ }^{512}$ One of the most well know zeolites is ZSM- 5 that as patented by Mobil Oil Company in 1975 [Zeolite Socony Mobil-5, an aluminosilicate zeolite with chemical formula is $\left.\mathrm{Na}_{n} \mathrm{Al}_{n} \mathrm{Si}_{96-n} \mathrm{O}_{192} \cdot 16 \mathrm{H}_{2} \mathrm{O}(0<\mathrm{n}<27)\right]$. It is used in the petroleum industry as a heterogeneous catalyst for hydrocarbon isomerization reactions. Typically, in the synthesis of zeolites, organic templates are often used and the framework is also slowly aged prior to its crystallization in a batch hydrothermal type process. ${ }^{512-514}$ Wakihara and co-workers reported the synthesis of ZSM-5 on the order of seconds in a continuous flow reactor ${ }^{515}$ via direct mixing of a precursor (at $90^{\circ} \mathrm{C}$ ) with superheated water at $\left(370^{\circ} \mathrm{C}\right.$ ), which gave a mixture in the range 240 to $300^{\circ} \mathrm{C}$ ). Other types of framework structures that can be made in flow reactors and that may have applications in catalysis (or gas storage) are for Metal-Organic Frameworks (MOFs), ${ }^{516}$ which are covered in more detail in section 10.4)

In the petrochemical industry, there is often a need to remove impurities that are naturally present in fuels. Due to increasingly stringent environmental regulations to limit sulfur in fuels, catalysts for removal of sulfur from crude oil and other derivatives, have been termed as deep-hydrodesulfurization (deep-HDS) catalysts. In order to achieve removal in a cost effective way, efficient and inexpensive deep-HDS catalysts are required. In one report by Aymonier and colleagues, a continuous flow process in supercritical water/alcohol mixtures (with a long residence time) was used to make $\mathrm{NiMoO}_{41}$ a preferred catalyst for deep-HDS (a Co analogue was also reported). ${ }^{31}$ By changing the type of alcohol in the solvent mixture, it was possible to tune BET surface area of the material; the as-made material contained a highly active rod like hydrate $\mathrm{NiMoO}_{4} \cdot 0.75 \mathrm{H}_{2} \mathrm{O}$ phase with a BET surface area up to $180 \mathrm{~m}^{2} \mathrm{~g}^{-1} \cdot 3^{1}$ Catalytic tests on a model desulfurization chemical 4,6-dimethyldibenzothiophene (4,6-DMDBT), showed better conversion of the reagent compared to commercial catalysts for the deep-HDS reaction. Furthermore, it was shown that there was an optimal $\mathrm{Ni}$ value and that the reaction could be performed at lower temperatures when using the nano-structured unsupported catalysts. ${ }^{31}$ 
Catalysis of Carbon Dioxide. Anthropogenic activities have led to a rise in greenhouse gases (including $\mathrm{CO}_{2}$ ) from ca. $280 \mathrm{ppm}$ before the industrial revolution (to ca. $390 \mathrm{ppm}$ in 2010), and which is predicted to be ca. $570 \mathrm{ppm}$ by the year $2100.5^{517}$ In tandem with $\mathrm{CO}_{2}$ storage and sequestration, conversion of $\mathrm{CO}_{2}$ to useful products such as formic acid, methane, methanol, is an appealing way to utilize $\mathrm{CO}_{2}$ as carbon source to fossil fuels. ${ }^{517}$ Recently, monodispersed $11 \mathrm{~nm}$ CuO nanoparticles (NPs) were synthesized via continuous hydrothermal synthesis and evaluated as heterogeneous catalysts for $\mathrm{CO}_{2}$ hydrogenation. 334 The nanoparticles were evaluated using a wide range of tests, e.g. they were characterized by diffuse reflectance infrared Fourier transform spectroscopy (DRIFTS), extended Xray absorption fine structure (EXAFS), X-ray diffraction (XRD), and catalytic testing, under $\mathrm{H}_{2} / \mathrm{CO}_{2}$ mixtures (temperature range 25 to $500{ }^{\circ} \mathrm{C}$. In situ DRIFTS data suggested surface formate species were present at temperatures as low as $70^{\circ} \mathrm{C}$, whilst DFT calculations suggested that reduction to $\mathrm{Cu}_{2} \mathrm{O}$ would facilitate formate formation. Indeed, In situ EXAFS supported a strong correlation between the formate peak intensity and $\mathrm{Cu}_{2} \mathrm{O}$ phase fraction. 334 The concurrent phase and crystallite size evolution were monitored by in situ XRD, which suggested the CuO NPs were stable in size before the onset of reduction, with $\mathrm{Cu}_{2} \mathrm{O}$ crystallites being formed from $130^{\circ} \mathrm{C}$. The formation of Cu metal from $190^{\circ} \mathrm{C}$, was followed by a rapid decrease of surface formate (and the detection of adsorbed CO from $250^{\circ} \mathrm{C}$ ); these results were in concordance with parallel heterogeneous catalytic tests (surface CO was observed over the same temperature range). Furthermore, $\mathrm{CH}_{4}$ was detected when the $\mathrm{Cu}$ metal phase was present, with a maximum conversion rate of $2.8 \%$ measured at $470^{\circ} \mathrm{C}$, suggesting an independent reaction pathway for the conversion of $\mathrm{CO}_{2}$ to $\mathrm{CH}_{4}$ and $\mathrm{CO}_{2}$ to $\mathrm{CO} .334$

As well as heterogeneous catalyzed reactions of $\mathrm{CO}_{2}$, some of the authors also recently reported to use of ultrafine $17 \mathrm{~nm}$ nanoparticles copper(II) oxide nanoparticles made via continuous hydrothermal synthesis for the electrocatalytic reduction of $\mathrm{CO}_{2}$ to formic acid was studied by evaluating nano-CuO / Nafion based inks on glassy carbon electrodes.335 In particular, the authors studied the influence of Nafion fraction on the Faradaic efficiencies and overpotential (for formic acid production), with the highest Faradaic efficiency being $61 \%$ (for ink with 25 wt $\%$ Nafion fraction), at a potential of $1.4 \mathrm{~V}$ vs. $\mathrm{Ag} / \mathrm{AgCl} .335$ This value for efficiency was significantly higher than the majority of comparable reports for copper catalysts in the literature and makes these materials potentially attractive to larger PEMFC-like designs for $\mathrm{CO}_{2}$ reduction, especially considering the scale up capabilities, green nature and ease of formulation of the continuous hydrothermal synthesis method. 335 
CHFS was used for the synthesis of graphene-oxide (GO) inorganic catalyst composites, denoted as Ce-La-ZrGO. ${ }^{518}$ The nanocomposite was synthesized from pre-mixed aqueous solution of cerium, lanthanum, zirconium nitrate and GO under alkaline conditions in flow. The resulting nanocomposite catalyst was heat-treated at either 773, 973 or $1173 \mathrm{~K}$ and then tested for the direct synthesis of propylene carbonate (PC) from cycloaddition of $\mathrm{CO}_{2}$ and propylene oxide (PO). ${ }^{518}$ The optimum reaction condition for the synthesis of PC was found at $443 \mathrm{~K}, 70$ bar and 10 $\%(\mathrm{w} / \mathrm{w})$ catalyst loading and the catalyst could be reused several times without losing catalytic activity. ${ }^{518}$

A number of other potential catalytic systems are of interest for the reduction of $\mathrm{CO}_{2}$ include the development of bio-inspired iron sulfide catalysts in the appropriate conditions. ${ }^{519}$ For example, greigite $\left(\mathrm{Fe}_{3} \mathrm{~S}_{4}\right)$ presents similar surface structures to the active sites found in many modern-day enzymes. It has recently been shown that particles of greigite can reduce $\mathrm{CO}_{2}$ under ambient conditions into chemicals such as methanol, formic, acetic and pyruvic acid. ${ }^{519}$ These results also lend support to the origin of life theory on alkaline hydrothermal vents (i.e. the formation of the building blocks of life from $\mathrm{CO}_{2}$ and other chemicals under conditions found on earth). Given the large number of reports now on the synthesis of sulfides in the continuous hydrothermal synthesis literature, ${ }^{115,122,370}$ there is potentially scope to develop new sulfide based low temperature $\mathrm{CO}_{2}$ reduction catalysts in the future.

\section{Other Oxidation / Reduction Catalysts.}

Several multi-component mixed metal oxide systems, including three phases of bismuth molybdate, namely $\mathrm{Bi}_{2} \mathrm{MoO}_{6}, \mathrm{Bi}_{2} \mathrm{Mo}_{2} \mathrm{O}_{9}$ and $\mathrm{Bi}_{2} \mathrm{Mo}_{3} \mathrm{O}_{12}$, have attracted considerable attention as oxidation catalysts..$^{520-521}$ Their synthesis typically involve post-synthesis treatment at elevated temperatures, which can make it difficult to control particle morphology and size in the products. Batch hydrothermal syntheses of bismuth molybdates generate crystalline products with high purity and narrow particle size distribution with synthesis temperatures in the range 140 to 280 ${ }^{\circ} \mathrm{C}$ and reaction times in the range 3 to $12 \mathrm{~h}$. In addition, judicious choice of reaction temperature, time, stoichiometry and $\mathrm{pH}$ are important for the controlled synthesis of specific phases. Although batch methods are widely used, the authors attempted to make finer bismuth molybdate particles (with higher surface areas) and perhaps greater control over particle size and shape. Depending on conditions, the authors successfully obtained $\alpha-\mathrm{Bi}_{2} \mathrm{Mo}_{3} \mathrm{O}_{12}$ and $\gamma^{-}$ $\mathrm{Bi}_{2} \mathrm{MoO}_{6}$ and found that simple variation in synthetic conditions and appropriate solution stoichiometry was sufficient to select the phase of the products. ${ }^{22}$ The CHFS reactions were as follows; bismuth nitrate and a basic solution of molybdic acid were each pumped at $10 \mathrm{~mL} \mathrm{~min}^{-1}$ in the reactor and had been diluted to achieve the desired 
metal stoichiometries (i.e. either a 2:1 or 2:3 atomic ratio of Bi:Mo) when they were mixed together at a low volume 1/8" tee-piece. ${ }^{522}$ At this point, an initial precipitate formed in situ was at $\mathrm{pH}$ 4. This precipitate was then brought into contact with a supercritical water feed being pumped at $20 \mathrm{~mL} \cdot \mathrm{min}^{-1}$ at $\leq 450^{\circ} \mathrm{C}$ and $24.1 \mathrm{MPa}$ in an countercurrent mixer, resulting in rapid crystallisation of the respective products. ${ }^{22}$ Depending on conditions, the materials were obtained as yellow powders in yields of 82 and $86 \%$ for the syntheses of $\alpha-\mathrm{Bi}_{2} \mathrm{Mo}_{3} \mathrm{O}_{12}$ and $\gamma-\mathrm{Bi}_{2} \mathrm{MoO}_{6}$, respectively.

Copper-manganese oxides are of considerable industrial interest as sustainable catalysts for the oxidation of CO at ambient temperature as well as for combustion of hydrocarbons or unwanted halide and nitrogen containing compounds.523 Adschiri et al. first reported the hydrothermal flow synthesis of $\mathrm{CuMn}_{2} \mathrm{O}_{4}$ nanocrystals at only $400{ }^{\circ} \mathrm{C}$ in a single step..$^{24} \mathrm{~A}$ feature of the research (as seen for some other heterometallic oxides) was that an initial mixed metal "hydroxide" precipitate or sol was first made, cleaned and then used as the precursor to be subsequently mixed with a sc-water feed in flow. ${ }^{524}$ In the report, it was found from XRD data that $\mathrm{CuMn}_{2} \mathrm{O}_{4}$ was a major phase with traces of $\mathrm{CuO}$, which may in part be due to the use of an initial inhomogeneous hydroxide precipitate in the mixture that was fed into the reactor or due to an excess of $\mathrm{Cu}$ in the initial precipitate that later evolved into CuO. Nevertheless, this general approach holds much promise to develop high surface area and more active oxidation catalysts.

NPs made via CHFS are ideally suited for use in a range of catalytic applications, particularly where a high surface area may benefit reaction rates. Due to the relatively higher surface energies of such small particles and extended defect surfaces, it is often observed that NPs are much more active catalysts at lower than expected temperatures, e.g. for the absorption of small molecules or mobility of lattice oxygen, etc. One of the most interesting recent reports to emerge on oxygen evolution catalysts made via continuous hydrothermal processes was on the synthesis of NiCo layered double hydroxide (LDH) nanoplates.525 LDHs are a family of $2 \mathrm{D}$ layered synthetic materials with stacked brucite-like $\mathrm{M}(\mathrm{OH})_{2}$ layers, where some of the $\mathrm{M}^{2+}$ ions are substituted by $\mathrm{M}^{3+}$ ions, resulting in positively charged host layers with charge-balancing anions between them. Several classes of LDHs are seen as inexpensive and versatile (allow doping) alternatives to more conventional precious metal-containing oxygen evolution reaction (OER) catalysts such iridium and ruthenium oxides. In the report by Jin et al. as-prepared NiCo LDH nanoplates showed high OER catalytic activity, particularly after increasing their surface area after chemical exfoliation (best samples gave a current density of $10 \mathrm{~mA} \mathrm{~cm}^{-2}$ at an overpotential of $367 \mathrm{mV}$ and a Tafel slope of $40 \mathrm{mV}$. $\left.\mathrm{dec}^{-1}\right) .5^{25}$ 
In closing, the topic of catalyst supports and non-precious metal supported catalysis is ever expanding, and there have been a number of developments. These new systems offer tunable reactivity, good abundance of starting materials (transition metals) and structural stability. Indeed, recent theoretical predictions indicate that complex perovskites (free of precious metals) can catalyze both ORR and OER. ${ }^{226}$ Such catalysts would also be of interest for ORR and OERs in not only heterogeneous catalysis but also fuel cell, electrolysis and energy storage applications. Thus, the development and use of nano-size and more active, defective/doped and high surface area catalysts that reduce or eliminate precious and scarce metals, is an area in which continuous hydrothermal processes should be explored in the future.

\subsubsection{Precious Metal Containing Particles for Catalysts}

In heterogeneous catalysis, precious metals such as Platinum Group Metals (PGMs) are often dispersed into a support matrix that can be either inert or indeed active within the catalytic process. By supporting the PGMs, it is also more likely to prevent sintering and lowering of the active surface. It is also possible for the PGM to be incorporated into the host matrix or for the precious metal to be near the surface, where dramatic changes oxygen mobility / lability can occur (and significantly lowering of the onset reaction temperature), due to the so called metal-support interaction. ${ }^{92}$ For example, formation of a mixed phase of $\mathrm{Pd}_{x} \mathrm{Ce}_{1-x} \mathrm{O}_{2-x-\delta}$ solid solution can form oxidized $\mathrm{PdO}_{x}(\mathrm{~s}) / \mathrm{Pd}-\mathrm{O}-\mathrm{Ce}(\mathrm{s})$ clusters, which generated highly reactive oxygen for the low temperature oxidation of $\mathrm{CO} .486$

Three Way Autocatalysts and Active Oxygen Applications. Amongst the most well-known catalysts are PGMs on ceria-zirconia based solid solutions for three way catalysts to convert $\mathrm{CO}$, hydrocarbons and $\mathrm{NO}_{x}$ into $\mathrm{CO}_{2}, \mathrm{H}_{2} \mathrm{O}$ and $\mathrm{N}_{2} .493,495,501$ Since the reaction rate is related to the surface area of the catalyst, the PGM particles must be as small as possible. Traditionally these catalysts are made either by impregnating a preformed support with a metal salt, or by co-precipitating the metal salt and support material. In both cases, the PGM NPs are formed on the surface of the support by heating the material under reducing atmosphere. It is also possible to make unsupported nanoparticles with PGMs under mildly reducing conditions in hydrothermal flow. Kimura and co-workers synthesized Pt from $\mathrm{H}_{2} \mathrm{PtCl}_{6}$ and $\mathrm{Na}_{2} \mathrm{PtCl}_{6}$, respectively, varying the metal salt concentration from 0.6 to $15 \mathrm{mM}$, with the mixing temperature in the range 373 to $563 \mathrm{~K}$ and the pressure ranging from 0.1 to $25 \mathrm{MPa} .{ }^{527}$ This paper, apart from being the first synthesis of nano-sized Pt by CHFS, was notable for the use of mixtures of ethanol and water as the solvent and 
for the use of PVP as a capping agent. The authors found that the size of the Pt particles was typically 2 to $3 \mathrm{~nm}$, where colloidal solutions were obtained, with the smallest particle size being $1.9 \mathrm{~nm}$ in pure water, however, 1:1 mixtures of water and ethanol afforded narrower particle size distributions. Using similar equipment and conditions, they produced Rh NPs in the range 2 to $5 \mathrm{~nm} \cdot{ }^{528}$ In these two papers, it was stated that the ethanol acted as a reducing agent, however, the ions were still reduced to the metal even in aqueous solution. This could be explained by the reducing effect of some decomposed PVP at the low temperatures used in these papers.

In the literature, a series of supported reduction catalysts were made via CHFS processes consisting of Pt, Pd and $\mathrm{Rh}$ supported onto $\mathrm{CeO}_{2}, \mathrm{ZrO}_{2}$ and $\mathrm{TiO}_{2}$, respectively. ${ }^{529}$ The supports were all made at $450{ }^{\circ} \mathrm{C}$ and $24.1 \mathrm{MPa}$, both with and without base for comparison. Selected samples were heat-treated in the temperature range 100 to $500{ }^{\circ} \mathrm{C}$ for 1 hour after drying. In addition, $\mathrm{NPs}$ of $\mathrm{CeO}_{2}, \mathrm{ZrO}_{2}$ and $\mathrm{TiO}_{2}$, respectively, were produced via $\mathrm{CHFS}$ and used as the supports for new catalysts via a further incipient wetness (IW) and heat-treatment step. In total, 53 individual catalysts were produced, tested for their activity for the hydrogenation of nitrobenzene to aniline and compared to other catalysts, which were used for commercial applications. It was found that the activities generally varied in the order $\mathrm{Pt}>\mathrm{Pd}>\mathrm{Rh}$ and $\mathrm{TiO}_{2}>\mathrm{ZrO}_{2}>\mathrm{CeO}_{2}$ for both the co-crystallized and incipient wetness samples.529 All the Rh catalysts were inactive for the hydrogenation of nitrobenzene to aniline, but interestingly, Rh metal NPs produced without a support, did have some activity. ${ }^{529}$ This may indicate that Rh was deactivated by the support. Heat-treatment up to $400^{\circ} \mathrm{C}$ did not make any difference to the activity of any of the above catalysts, indicating that any surface groups present on the active metal, did not significantly affect the activity of the catalysts. Heattreatment at $500^{\circ} \mathrm{C}$, tended to reduce catalytic activity, suggesting the onset of sintering and loss of surface area. TEM analysis showed that in catalysts where the noble metal cation was larger than the support metal cation, the catalysts were phase separated, i.e. there were noble metal particles clearly visible on the TEM image of the support.529 $\mathrm{Rh}$ dispersed over $\mathrm{Ce}_{\mathrm{x}} \mathrm{Zr}_{1-\mathrm{x}} \mathrm{O}_{2}$ (made via CHFS) was also prepared by $\mathrm{lhm}$ and co-workers.530 The material was used for catalytic reduction of $\mathrm{NO}$ by $\mathrm{CO}$, which showed enhanced reducibility for Rh and superior catalytic performance.

There are examples of CHFS reactions directly producing supported catalysts, by co-precipitating an active PGM with a support phase simultaneously in $\mathrm{sc}-\mathrm{H}_{2} \mathrm{O}$. Galkin et al. produced a number of potential catalysts including Rh and $\mathrm{Pd}$ on $\mathrm{ZrO}_{2}$ and $\mathrm{TiO}_{2}$, respectively. ${ }^{369}$ They found from TPR studies that pure $\mathrm{ZrO}_{2}$ made via CHFS, displayed three reduction peaks below $1273 \mathrm{~K}$ (bulk $\mathrm{ZrO}_{2}$ cannot normally be reduced at temperatures below $1000^{\circ} \mathrm{C}$ ). Three 
steps of reduction for $\mathrm{ZrO}_{2}$ made via CHFS were observed below $1000^{\circ} \mathrm{C}$, and the first reduction peak was observed at $232^{\circ} \mathrm{C} .369$

When rhodium was added into the in the $\mathrm{ZrO}_{2}$ catalyst made via CHFS, the consumption of hydrogen increased. Interestingly, when palladium rather than rhodium was used, hydrogen uptake began at room temperature, and desorption of hydrogen was at ca. $70^{\circ} \mathrm{C}$. It was suggested that a possible advantage of CHFS was the higher (by at least an order of magnitude) concentration of $\mathrm{Zr}^{3+}$ ions (compared to the material prepared by more conventional processes), which are regarded as the active sites in the Fischer-Tropsch processes..$^{509}$

The authors thus, believe that there is potential to utilise the CHFS method for the development of next generation TWC's, particularly in areas where the surface area and microstructure of the ceria-zirconia based solid solution can be controlled to reduce sintering and to enhance the presence of defect of highly mobile oxygen. In many catalytic supports, the effect of ageing or a heat-treatment can result in a loss of surface area or pore volume, which can reduce activity. In a report by Kim and co-workers, it was shown that supercritical flow synthesis of $\mathrm{Ce}_{0.65} \mathrm{Zr}_{0.35} \mathrm{O}_{2}$ supported catalysts showed reduced loss of activity after redox ageing (treatment of the solids with $\mathrm{H}_{2}$ and $\mathrm{O}_{2}$ in turn at $1000^{\circ} \mathrm{C}$ for $6 \mathrm{~h}$ ), as a result of less sintering. This was in contrast to a more conventional room temperature co-precipitated material. ${ }^{367}$ In the report, the ceria-zirconia materials were made and then heat-treated for 12 h. For the conventionally co-precipitated materials, the ceria-zirconia mixed oxide was obtained by heat-treating the initially formed hydroxide in an air flow at $700^{\circ} \mathrm{C}$ for $5 \mathrm{~h}$. After redox ageing of the supercritical and conventionally made ceria-zirconia catalysts, it was shown that the OSC for the latter decreased from $700 \mu \mathrm{mol}-\mathrm{O}_{2} \cdot \mathrm{g}^{-1}$ to $330 \mu \mathrm{mol}-$ $\mathrm{O}_{2} \cdot \mathrm{g}^{-1}$, while the OSC was largely retained for former material, due to less sintering on aging (original value 680 $\left.\mu \mathrm{mol}-\mathrm{O}_{2} \cdot \mathrm{g}^{-1}\right)$. Rh-loaded ceria-zirconia mixed oxides were also prepared using incipient wetness method, followed by drying and heat-treatment in air at $600^{\circ} \mathrm{C}$ for $3 \mathrm{~h}$. The Rh loaded aged materials, showed excellent OSC retention on ageing, which was in contrast to the ceria-zirconia samples made conventionally. ${ }^{367}$ Such considerations about the effects of sintering and ageing are critical in designing future automotive catalysts.

To some extent the catalytic activities of supported catalysts are highly dependent on the nature of the dispersion of active species and the interaction between the active species and supports (or materials that reduce sintering), where the former can also profoundly affect the latter. The authors described the preparation of $\mathrm{Ce}_{0.6} \mathrm{Zr}_{0.3} \mathrm{La}_{0.05} \mathrm{Y}_{0.05} \mathrm{O}_{2-\delta} / \mathrm{\gamma}-\mathrm{Al}_{2} \mathrm{O}_{3}(\mathrm{CZA})$ and $\mathrm{Pd} / \mathrm{Ce}_{0.6} \mathrm{Zr}_{0.3} \mathrm{La}_{0.05} \mathrm{Y}_{0.05} \mathrm{O}_{2-\delta} / \mathrm{\gamma}-\mathrm{Al}_{2} \mathrm{O}_{3}(\mathrm{PdCZA})$ composite oxides that were prepared in one step as nanopowders using CHFS..$^{2}$ The team investigated the homogeneities, thermal stabilities 
and catalytic activities of the samples after a $10 \mathrm{~h}$ heat-treatment at $1000^{\circ} \mathrm{C}$ in air (accelerated ageing). It was observed that this accelerated ageing changed the BET surface areas for the CZA sample from ca. 110 to ca. $36 \mathrm{~m}^{2} \mathrm{~g}^{-1}$ (which was about three-fold higher than that of aged ceria-zirconia solid solution sample at ca. $13 \mathrm{~m}^{2} \mathrm{~g}^{-1}$ ). Interestingly, accelerated ageing did not significantly affect the $\mathrm{T}_{90}$ (time taken to decompose $90 \%$ of the gas) for $\mathrm{NO}$ and $\mathrm{C}_{x} \mathrm{H}_{y}$ during three-way catalytic activity measurements (from ca. 265 to ca. $310^{\circ} \mathrm{C}$ after ageing)..$^{22}$ This implied a remarkably high thermal stability of the PdCZA sample made via the CHFS route due to the co-precipitation with zirconia (see figure 31). Such an approach to get intimate mixtures of two or more phases, which can then be exploited (for solid state chemistry or prevent sintering) is an attractive benefit for CHFS as will be seen later for example in highly conducting composite Ni / YSZ cermets 357 and for solid state chemistry of difficult to make heterometallic oxides. ${ }^{38,498,531}$

Figure 31

The interaction of PGM atoms or particles and a support can result in Strong-Metal Support Interaction (SMSI) that can confer extraordinary properties for a large set of technologically important applications, e.g. they could increase oxygen mobility and reducibility for materials, which profoundly enhances their activities for catalytic reactions. SMSI features have been studied for CHFS made samples when the PGM was in intimate contact with the support metal oxide.532 The authors used a spherical aberration corrected scanning transmission electronmicroscope (Cs-corrected STEM) to study a SMSI feature between Pt and $\mathrm{CeO}_{2}$ hybrid oxides obtained from CHFS. ${ }^{52}$ From the Cs-corrected STEM image, Pt atoms were observed to be interlocked with the Ce atoms (see Figure 32) after they were co-precipitated in flow using sc- $\mathrm{H}_{2} \mathrm{O}$. The $\mathrm{SMSI} \mathrm{Pt} \mathrm{/} \mathrm{CeO}_{2}$ hybrid oxides showed remarkable reducibility that yielded a much higher activity for toluene oxidation compared to "traditionally prepared" Pt / $\mathrm{CeO}_{2}$. In the report, the authors also showed that addition of formic acid in a CHFS reactor could effectively adjust the valence of $\mathrm{Pt}$, which led to further enhanced SMSI and catalytic activity. ${ }^{532}$

Figure 32 
ultimately solar-driven) splitting of seawater into hydrogen and an oxidised chloride $\left(\mathrm{Cl}_{2} / \mathrm{NaOCl}\right)$. The oxidised chloride can be stored either as $\mathrm{Cl}_{2}$, or hypochlorite (depending on reaction $\mathrm{pH}$ ), thereby providing an inexpensive and portable route to chlorinated water or disinfectant production, whilst the useful by-product of hydrogen gas, could be used to provide heat / electricity at a later date (by burning or via a fuel cell, respectively). Mixtures of ruthenium and titanium oxides (similar to those used in the industrial production of chlorine through the chlor-alkali process ${ }^{533}$ ), were produced by the authors using continuous Hydrothermal synthesis and tested as catalysts for both oxygen gas and chlorine gas evolution. A rapid colourimetric screening test was used, where Ce(IV) [yellow] was used as a sacrificial oxidant and was reduced to Ce(III) [colourless] in a brine solution. ${ }^{534-535}$ CHFS nanopowders were then compared against a high performing $\mathrm{RuO}_{2}$ (Ru-Adams) material, synthesized using a modified Adams type molten salt approach. Improved catalytic activity resulted; this was due to the higher surface area of the materials. Improved selectivity towards chlorine (versus oxygen) gas evolution (up to ca. $92 \% \mathrm{Cl}_{2}$ yield) was seen for samples with low ruthenium content. ${ }^{334}$ Figure 33 a shows a typical UV/Vis absorbance decay profile showing the reduction of $\mathrm{Ce}(\mathrm{IV})$ to $\mathrm{Ce}(\mathrm{III})$ at $430 \mathrm{~nm}$. In this case, three serial injections of $90 \mathrm{~mL}$ of $0.1 \mathrm{M} \mathrm{Ce}(\mathrm{IV}$ ) (aq.) were added to a stirred dispersion of catalyst that was ultrasonicated. The most promising Chloride Oxidation Catalyst $(\mathrm{ClOC})$ sample made in CHFS (containing 50 at\% Ru), was used to produce an anode on transparent conducting glass and then used in a demonstrator device for solar brine splitting that incorporated a solar cell. $534-535$

The $\mathrm{ClOC}$ electrode was shown to produce hydrogen and chlorine when placed in the cell with acidified brine (under illumination). Figure $33 \mathrm{~b}$ shows the results for the photo-cleavage of brine using a 50 at $\%$ Ru anode and platinum mesh cathode, coupled to a Si solar cell operating at $1.89 \mathrm{~V}$, in $0.5 \mathrm{M} \mathrm{H}_{2} \mathrm{SO}_{4}$ and $2 \mathrm{M} \mathrm{NaCl}$. The figure shows (i) the predicted temporal $\mathrm{H}_{2}$ yield, the measured temporal $\mathrm{H}_{2}$ yield (based on $\mathrm{GC}$ measurements) the final $\mathrm{Cl}_{2}$ yield (measured using a $\mathrm{KI}$ trap). The corresponding $\mathrm{Cl}_{2}$ yield was also shown to improve when a potentiostat set at $1.44 \mathrm{~V}$ was used instead of the solar cell $\left(80 \% \mathrm{Cl}_{2}\right.$ yield obtained due to the less competition with oxygen production from water splitting at this potential).

Figure 33 
This work has effectively shown that highly selective brine splitting catalysts can be developed at reduced Ru content, which can be run at low current densities (compared to the commercial chlor-alkali process533), which offers the potential for personal or smaller scale domestic production of hydrogen or chlorine or perchlorate. The authors are currently trying to better understand the location of the Ru and Ti atoms within the structures of the CLOC catalysts and believe that there is still further potential to utilise the (high throughput) CHFS method for the development of other more sustainable $\mathrm{ClOC}$ catalysts in the future (i.e. with little or no Ru). Notably, these Ru-Ti oxide oxidation catalysts should also be of interest as supercapacitor materials. 535 The results of these endeavours will be reported in due course.

\section{8.o DEVICES (ENERGY HARVESTING / CONVERSION / FUELS)}

As we have already seen, the continuous hydrothermal process can make catalytic and active nanomaterials. Some of these catalysts and related materials can also be used in energy conversion, harvesting or related devices (e.g. as electrode catalysts or components in fuel cells or solar cells) or for devices that create fuels or separate oxygen from the air. Some of these devices are critical to improving energy efficiencies in the future or for reducing reliance on fossil fuels for the benefit of mankind.

\subsection{Solid Oxide Fuel Cell Materials (Including Mixed Ion Conductors) and Related Technologies}

Fuel cells are one of the major commercial applications of mixed ion conductors and PGMs, attracting tremendous interest because of their as yet unfulfilled potential in transport and domestic and large scale power generation. ${ }^{336}$ They can offer a combination of high efficiency for direct conversion of chemical energy into electrical energy, producing much lower emissions of $\mathrm{S}$ and $\mathrm{N}$ oxides, and reducing $\mathrm{CO}_{2}$ and hydrocarbon pollutants. Altogether, they offer significant environmental advantages over conventional power generation methods. With relevance to the applications of different types of fuel cells, the authors will initially focus on Solid Oxide Fuel Cells (SOFCs). ${ }^{38,91,357,498,}$ 537-538 There have been some initial reports on the CHFS of nanomaterials that might be suitable as components in SOFCs. The authors envisage that other fuel cells such as polymer electrolyte membrane fuel cells (PEMFCs), might 
also be able to benefit from the use of metal oxide or metal NPs made via CHFS for improving performance (for oxygen reduction or oxygen evolution reactions, respectively).

SOFCs are particularly of interest for combined heat and power applications for domestic and large-scale power generations. A SOFC generally consists of an anode where the fuel (e.g. CO and hydrogen) can react, an oxygen ion electrolyte (oxide ion conductors, such as yttria stabilized zirconia, YSZ, that is electronically insulating) and a cathode where the oxygen is reduced to oxygen anion $\left(\mathrm{O}^{2-}\right)$. The oxygen anions in a SOFC pass from the cathode, through electrolyte under electrical load and react with the fuel at the anode. A basic SOFC process produces $\mathrm{CO}_{2}$ and water, electricity and heat. An excellent review by Ormerod is highly recommended for those wishing to learn more about this subject. ${ }^{56}$ There are many opportunities to use NPs made via continuous hydrothermal (or of course other methods) in the development of SOFC materials application, particularly in the development of anode, cathode, interconnect and electrolyte materials.

\section{Electrolyte Materials for SOFCs.}

By far the most popular material for this component is yttria-stabilized zirconia $\left(\mathrm{ZrO}_{2}\right)_{1-x}\left(\mathrm{Y}_{2} \mathrm{O}_{3}\right)_{x}$. The doping of a $3^{+}$ cation of $\mathrm{Y}$ into the $4^{+} \mathrm{ZrO}_{2}$ oxide structure, leads to oxygen ion vacancies (including single vacancies and vacancy pairs), which will depend on the yttria concentration and in turn affect the ionic conductivity of the material under operating conditions.

For electrolyte materials (e.g. YSZ), the fabrication of these materials for SOFC device stacks has its difficulties. The YSZ ceramic ink is often screen printed as one of several layers and then the whole device has to be fired at high temperatures $>1200^{\circ} \mathrm{C}$ to give a gas tight YSZ barrier between the electrode layers. As such, there is interest in reducing the sintering temperature of the YSZ itself and producing gas tight, fully dense YSZ layers after sintering. NPs could provide a route to resolve exactly these issues, particularly using continuous hydrothermal technology. Investigations by the authors on CHFS-made YSZ NPs (at ca. $5 \mathrm{~nm}$ ), revealed that the onset of sintering could be reduced from ca. $1300^{\circ} \mathrm{C}$ (for bulk YSZ) to ca. $1070^{\circ} \mathrm{C}$, which is in keeping with the literature reports of the significant lowering of the onset of sintering temperature for nano-sized YSZ made via other methods. ${ }^{539}$ Such a lowering of sintering temperature was attributed to the high surface energy of NPs.

Reports on the syntheses of YSZ materials made using the continuous hydrothermal technique, include reports by Hayashi et al. ${ }^{288}$ and Teja et al. ${ }^{668}$ where the latter suggested that CHFS manufacture of YSZ NPs should avoid 
$\mathrm{KOH}$ (as it can damage zirconia electrolytes). In the former report, it was found that particles made in the temperature range 300 to $400{ }^{\circ} \mathrm{C}(\mathrm{pH}$ range 1 to 11$)$, it was found that crystallite sizes were in the size range 4 to $6 \mathrm{~nm}$ (estimated from $\mathrm{BET}$ ) irrespective of solution $\mathrm{pH}$ and temperature. Furthermore, it was observed that $\mathrm{Y}$ conversion increased with increase in solution $\mathrm{pH}$ and temperature and the correct stoichiometric $\mathrm{Y}: \mathrm{Zr}$ solid solution was obtained at a $\mathrm{pH}>8 .{ }^{288}$

Key to the performance of YSZ in a SOFC is the composition and method of materials manufacture and how this affects lifetime and operation. Degradation can happen over time and this is generally due to, two mechanisms; (i) short-range ordering of oxygen vacancies and (ii) precipitation of a tetragonal $\mathrm{ZrO}_{2}$ phase from the $\mathrm{cubic} \mathrm{ZrO}_{2}$ matrix. Thus, in the future, it could be useful to correlate long-term stability to the methods of synthesis.

Anode Materials for SOFCs. SOFC anode materials are required to electrically conduct and withstand the reducing conditions of the fuel and the transport of oxygen ions across the electrolyte. In order to achieve these dual properties, Ni-YSZ cermet (a composite of metal and ceramic) is of interest as it can achieve suitable properties at >30 vol.\% Ni content. The high level of Ni coupled to thermal mismatch with the ceramic part, can lead to stress buildup and fracture of the anode during the fabrication process and in operating during temperature cycling. The UCL authors postulated that it may be possible to reduce the amount of Ni metal required to achieve reasonable conductivity and improved thermal characteristics if the Ni and YSZ can be made as a sintered intimate co-precipitate. In collaboration with Imperial College fuel cell group of Nigel Brandon, ${ }^{100}$ the UCL authors investigated nano-coprecipitates of Ni and YSZ that were made by continuous hydrothermal. A rapid consolidation technique known as spark plasma sintering (SPS) was used to rapidly densify the material in a very short timeframe and to in situ reduce the $\mathrm{NiO}$ to Ni during sintering. The continuous hydrothermal method successfully produced NPs in which the Ni was evenly distributed in the YSZ (see Figure 34), which effectively improved the thermal stability and conductivity. Electrical conductivity tests showed that the CHFS made material after SPS produced a Ni/YSZ cermet with higher than expected electrical conductivity. The 24 vol\% Ni-containing material had an electrical conductivity of $\sim 200 \mathrm{~S} . \mathrm{cm}^{-1}$ at the fuel cell operating temperature, which corresponded to an effective conductivity of $\sim 117 \mathrm{~S} . \mathrm{cm}^{-1}$ if a porosity of 30 vol\% was to be introduced. This significantly improved electrical conductivity at low Ni content $(<30$ vol\%) means that the layer is likely to be more redox cycle tolerant and have better thermal expansion compatibility with the ceramic electrolyte. 
Figure 34

In the fabrication of printed solid oxide fuel cell devices, nanoparticles can be formulated with larger micron sized particles because the former will sinter at relatively lower temperatures, whilst the latter will undergo less shrinkage upon densification. SOFC anodes based in Ni containing ceria-gadolinia (CGO) were made using impregnation of nickel nitrate solution on CGO scaffolds. ${ }^{337}$ The scaffolds were produced from inks containing a mixture of CHFSmade CGO, larger size commercial CGO and polymeric pore formers. Mechanically robust scaffolds were made by heat-treatment at either 1000 or $1300^{\circ} \mathrm{C}$ (onset of sintering for the NPs was in the range 900 to $1000^{\circ} \mathrm{C}$ ). $537 \mathrm{Impreg}$ nated scaffolds were used to produce symmetrical fuel cells, with the lower temperature heat-treated scaffolds showing improved gas diffusion, but poorer charge transfer. Using these scaffolds, lower temperature heat-treated fuel cells of Ni-CGO / $200 \mu \mathrm{m}$ YSZ / CGO-LSCF, performed better at $700^{\circ} \mathrm{C}$ (and below) in hydrogen, and performed better at all temperatures using syngas, with power densities of up to $0.15 \mathrm{~W} . \mathrm{cm}^{-2}$ at $800{ }^{\circ} \mathrm{C} .537$ The authors suggest that in this "bricks and mortar" approach, the nanoceramics were the binding component for bringing together larger micron sized particles for the manufacture of robust SOFC anodes.

Cathode Materials for SOFCs. For solid oxide fuel cell cathode materials, there is a requirement for mixed electrical and oxygen ion conductivity. ${ }^{536}$ Such materials are effective catalytically active materials that could in theory have appeared in other sections of the review (e.g. as oxidation catalysts). There are of course specific points that arise for such materials and these will be discussed herein for the development of fuel cells. For example, it important that we understand the role of defects in affecting the ionic and electronic properties; for example, under oxygen rich conditions, the defect chemistry may favour p-type conductivity, with cation vacancies playing a crucial role. In contrast, in oxygen poor conditions, it could be that $\mathrm{O}^{2-}$ vacancies dominate, leading to $\mathrm{n}$-type conductivity.

Many mixed ion conductors are heterometallic layered oxides and are inherently difficult to make. An example of such materials includes the (La-Ni-O) binary oxide system that forms a series of compounds with the general formula $\mathrm{La}_{n+1} \mathrm{Ni}_{n} \mathrm{O}_{3 n+1}$. As a consequence of their layered framework, these phases can accommodate a range of nonstoichiometry. Changes in the La:Ni ratio for the series, can lead to different ionic and electronic behaviours. For ex- 
ample, the slightly oxygen deficient $n=2$ and $n=3$ compounds $\left(\mathrm{La}_{3} \mathrm{Ni}_{2} \mathrm{O}_{7-\delta}\right.$ and $\mathrm{La}_{4} \mathrm{Ni}_{3} \mathrm{O}_{10-\delta}$, respectively) can possess a good balance of both ionic and electronic conductivity, due to the increasing number of perovskite layers (between each set of LaO rocksalt layers). Such materials are considered possible candidates for oxide-ion and electronic conducting cathode materials in SOFC devices; however, their syntheses are laborious and energy intensive. Typically, the literature shows that $\mathrm{La}_{4} \mathrm{Ni}_{3} \mathrm{O}_{10-\delta}\left(\right.$ or $\mathrm{La}_{3} \mathrm{Ni}_{2} \mathrm{O}_{7-\delta}$ ) can be made by heating the corresponding metal oxide powders at $1100^{\circ} \mathrm{C}$ over 4 to 5 days with intermittent regrinding (homogenization) steps..$^{540-541}$

The UCL authors investigated how "difficult to make" $\mathrm{La}_{n+1} \mathrm{Nin}_{n} \mathrm{O}_{3 n+1}$. materials could be made more efficiently using the NP co-precipitates; co-precipitates of lanthanum and nickel hydroxides were prepared with different La:Ni ratios in a continuous hydrothermal system. These co-precipitates were subsequently heat-treated (only using $6 \mathrm{~h}$ ) in air in the corresponding La:Ni ratios, for direct syntheses of phase pure $\mathrm{La}_{4} \mathrm{Ni}_{3} \mathrm{O}_{10-\delta}$ and $\mathrm{La}_{3} \mathrm{Ni}_{2} \mathrm{O}_{7-\delta}$, respectively. This result was the fastest and first reported direct syntheses of $\mathrm{La}_{4} \mathrm{Ni}_{3} \mathrm{O}_{10-\delta}$ and $\mathrm{La}_{3} \mathrm{Ni}_{2} \mathrm{O}_{7-\delta}$ from any precursor. It was speculated that such successful direct syntheses of $\mathrm{La}_{4} \mathrm{Ni}_{3} \mathrm{O}_{10-\delta}$ and $\mathrm{La}_{3} \mathrm{Ni}_{2} \mathrm{O}_{7-\delta}$ by not using any regrinding steps, was due to the high intimacy of mixing achieved between the respective metal hydroxides for the initial coprecipitates (made in CHFS). This facilitated efficent mass transfer during the subsequent heat-treatments, to give phase pure products after only $6 \mathrm{~h}$. Compared to more conventional room temperature batch co-precipitation syntheses (where several phases can be formed at different times during the reaction), the CHFS method generally ensures both initial phases are rapidly formed and in the worst case, they are intimately mixed (if not doped). This allows the limitations of insufficient mass transfer to be overcome (upon heating) that would normally exist for less homogenous or larger particle mixtures.

In order to better understand the solid state transformations that were occurring between the lanthanum and nickel hydroxide NPs, the authors investigated an in situ VT-XRD study. ${ }^{53^{8}}$ Due to the use of a platinum strip resistive heater, the results were not consistent with previous results obtained via muffle furnace heating of the precursors, (likely to be due to differences in oxygen partial pressures). However, notably the synthesis of $\mathrm{La}_{2} \mathrm{NiO}_{4-\delta}$, was achieved directly from the hydroxide nano-precipitates at a heat-treatment temperature of only $700^{\circ} \mathrm{C}$ (both in air and argon), which was the lowest synthesis temperature for this phase reported to date.

An automated robotic CHFS system RAMSI, was used to make $\mathrm{La}_{4} \mathrm{Ni}_{3-x} \mathrm{Fe}_{x} \mathrm{O}_{10}$ phases $(x=0.0$ to 3.0 ) directly from CHFS from nano-precipitates without comminution. RAMSI incorporated an automated (rather than manual) High Throughput Continuous Hydrothermal $(\mathrm{HiTCH})$ flow synthesis reactor, as shown in figure 35a. The CHFS samples 
were split into two identical cloned libraries, which were heat-treated (at 1348 or $1573 \mathrm{~K}$ for $12 \mathrm{~h}$ ) to find the right conditions to bring about solid-state transformations. The heat-treated samples from each library were embedded into a wellplate and analysed by powder X-ray diffraction (to study trends in phase behavior). ${ }^{542}$ Several new compositions for phase-pure Ruddlesden Popper type $\mathrm{La}_{4} \mathrm{Ni}_{3-x} \mathrm{Fe}_{x} \mathrm{O}_{10}$ structures were identified and their DC electrical conductivities, measured. 542

A similar but manual HiTCH flow synthesis approach was used for other doped lanthanum nickelates. ${ }^{443}$ In this HiTCH flow synthesis approach; each metal salt composition was introduced manually into the apparatus using $\mathrm{P}_{\mathbf{1}}$, whereupon it mixed with $1 \mathrm{M} \mathrm{KOH}$ solution $\left(\mathrm{P}_{2}\right)$ in a T-piece mixer. The reaction mixture of $\mathrm{KOH}$ and metal salt was then mixed with a stream of supercritical water in a $1 / 4^{\prime \prime}$ outer diameter co-current mixer (or other mixer as appropriate), whereupon nano-sized metal oxide co-precipitates were made. After precipitation, the NPs were cooled, recovered and then cleaned up. Initial libraries were made sequentially using HiTCH flow synthesis to co-precipitate a mixture of intimately mixed $\mathrm{La}$ and $\mathrm{Ni}$ oxides in the presence of additional metal ion dopants $(\mathrm{M}=\mathrm{V}, \mathrm{Cr}, \mathrm{Mn}, \mathrm{Fe}, \mathrm{Co}$, $\mathrm{Ni}$, and Al).543 A total of eight precursors were made to achieve a nominal formula after heat-treatments of $\mathrm{La}_{4} \mathrm{Ni}_{2.7} \mathrm{M}_{0.3} \mathrm{O}_{10-\delta}$ (where $\mathrm{M}=\mathrm{V}, \mathrm{Cr}, \mathrm{Mn}, \mathrm{Fe}, \mathrm{Co}, \mathrm{Ni}$, and $\mathrm{Al}$ ).

In order to elucidate the correct thermodynamic temperature to obtain phase pure materials, each set of precursors was divided into three cloned libraries. The libraries were heat-treated for 12 hours at either 1348,1448 or 1548 $K$, respectively, to bring about solid-state transformations (see Figure 35 b). 543 This gave an $8 \times 3$ set of samples $=24$ samples of which 12 samples were phase pure according to PXRD (four compositions previously unknown were identified). The DC conductivity of seven of the phase pure samples was tested, which revealed that undoped $\mathrm{La}_{4} \mathrm{Ni}_{3} \mathrm{O}_{10-\delta}$ had the highest conductivity of $241 \mathrm{~S} \cdot \mathrm{cm}^{-1}$ at $523 \mathrm{~K}$ (disk density $=69 \%$ ). All doped samples were less conductive, most likely due to hole doping of the valence band as a result of too high M(III) content. The greatest conductivity of the doped samples was for the Fe doped sample $\mathrm{La}_{4} \mathrm{Ni}_{2.7} \mathrm{Fe}_{0.3} \mathrm{O}_{10-\delta}$ (with a conductivity of $100 \mathrm{~S} . \mathrm{cm}^{-1}$ at $573 \mathrm{~K}$ ) for a sample of $61 \%$ density (see Figure $35 \mathrm{c}$ ). ${ }^{543}$ The least conductive was the Mn doped one, $\mathrm{La}_{4} \mathrm{Ni}_{2.7} \mathrm{Mn}_{0.3} \mathrm{O}_{10-\delta}$, which had the conductivity of $51 \mathrm{~S} \cdot \mathrm{cm}^{-1}$ at $573 \mathrm{~K}$ (for a sample of $61 \%$ density).

Figure 35 
Other materials that might be of interest as mixed ion conductors in SOFCs include the $\mathrm{La}_{2} \mathrm{CuO}_{4}$ perovskite. This was obtained as an intimate mixture of $\mathrm{La}(\mathrm{OH})_{3}$ and $\mathrm{CuO}$ to give single phase $\mathrm{La}_{2} \mathrm{CuO}_{4-\delta}$ after a single $5 \mathrm{~h}$ heattreatment at $600^{\circ} \mathrm{C} .{ }^{140}$ Such a material is in fact of interest as an oxidation catalyst so other discussion of the properties of this compound is given in the section on catalysts.

SOFC cathodes are therefore, still very much an area, which warrants future research. Presently, attention is focused on perovskite structures (which are based in the $\mathrm{ABO}_{3}$ structure such as $\mathrm{LalnO}_{3}$ or $\mathrm{LaCoO}_{3}$ that can accommodate large amounts of anion vacancies (that can enhance oxygen conductivities. In order to increase the population of mobile oxygen vacancies, researchers can substitute some of the trivalent cations for divalent ones (so charge compensation occurs in the anions). Future, research will no doubt focus on optimising the ionic conductivity with the dopant for perovskite systems. CHFS might be a valuable tool for making such doped materials quickly and reliably in order to develop more active materials that can work in lower temperatures (intermediate) SOFCs that operate near to $600{ }^{\circ} \mathrm{C}$ rather than $1000^{\circ} \mathrm{C}$.

8.2 High Temperature Membrane Technologies. Mixed ion conductors have a range of applications in oxygen separation membranes 544 for making pure oxygen or for converting gases into hydrocarbon fuels.

In the aforementioned La-Ni-oxides, ${ }^{38,91,538}$ the structures were based on doped / undoped alternating perovskite and rock-salt layers, which alter the balance of electronic and ionic conductivity. However, the perovskite structure itself, can be doped to make mixed ion conductors, which contain large amounts of anion vacancies (that can enhance oxygen conductivities (see above for SOFC cathodes). Such materials are of interest in solid oxide fuel cell cathodes as well as oxygen separation membranes 544 for making oxygen gas or for conversion of methane to syngas, which also needs oxygen (which is itself then used to make liquid fuels downstream).

In $\mathrm{ABO}_{3}$ perovskite-type oxides, the $\mathrm{A}$-site tends to be occupied by large alkaline earth metals and the $\mathrm{B}$-site by lanthanides and smaller first row transition elements. Doping heterovalent cations of a similar size into the $\mathrm{ABO}_{3}$ structure, results in oxygen vacancies being created, consequently, this alters the ionic and electronic conductivity of the end materials. In 2008, Hayashi et al. reported the continuous hydrothermal synthesis of perovskite $\mathrm{Ca}_{0.8} \mathrm{Sr}_{0.2} \mathrm{Ti}_{1-}$ ${ }_{x} \mathrm{Fe}_{\times} \mathrm{O}_{3-\delta}(\mathrm{CTO}) \mathrm{NPs}$, in which $\mathrm{Ca}^{2+}$ and $\mathrm{Ti}^{4+}$ sites were partially doped by $\mathrm{Sr}^{2+}$ and Fe-ions, respectively. 433 They found that a single-phase CTO materials were obtained at $673 \mathrm{~K}(30 \mathrm{MPa})$ and under high pH only, with particles typically ca $20 \mathrm{~nm}$ and BET surface areas in the range 70 to $90 \mathrm{~m}^{2} \mathrm{~g}^{-1}$. At lower Fe contents, it was possible to manufacture 
phase pure CTO under subcritical conditions, otherwise supercritical conditions were required in the flow system. In 2012, Hasyahi et al. then used similar perovskite materials to make asymmetric membranes to produce synthesis gas from methane. ${ }^{431}$ The 20 micron thick membranes were made using $\mathrm{Ca}_{0.8} \mathrm{Sr}_{0.2} \mathrm{Ti}_{1-x} \mathrm{Fe}_{x} \mathrm{O}_{3-\delta}(\mathrm{CTO})$ perovskite (with Ni being supported on it) powders. The performance of the membrane was for the partial oxidation of methane, gave conversions of methane and selectivity to $\mathrm{CO}$ of $82.3 \%$ and $99.8 \%$ at $1173 \mathrm{~K}$, respectively. This was significantly improved compared with the unsupported solid disk type membrane with ca. 500 micron thickness. ${ }^{431}$

Composite cermets using CHFS-made NPs have also been developed for more sustainable low-metal-content oxygen separation membranes. ${ }^{544}$ A dense composite of silver and $\mathrm{Ce}_{0.8} \mathrm{Sm}_{0.2} \mathrm{O}_{2-\delta}(\mathrm{Ag}-\mathrm{CSO})$ was manufactured from ceramic NPs coated by electroless deposition of silver. At $700^{\circ} \mathrm{C}$, a $1 \mathrm{~mm}$-thick membrane of the composite, delivered an excellent oxygen permeation rate from air with a value of $0.04 \mu \mathrm{mol} . \mathrm{cm}^{-2} . \mathrm{s}^{-1}$, using argon as the sweep gas and $0.17 \mu \mathrm{mol} . \mathrm{cm}^{-2} \cdot \mathrm{s}^{-1}$ using hydrogen. ${ }^{544}$ The low sintering temperature of the CSO NPs, allows the use of Ag rather than Pt or Pd and reduced the amount of metal needed for electronic conductivity to just 5.6 vol\%, which was much lower than any value reported in the literature (see Figure 36). Oxygen diffusivity measurements confirmed that the oxygen migration remained high in the composite, with an increase in surface exchange coefficient of three orders of magnitude over Gd-doped ceria. The ease of membrane fabrication, combined with encouraging oxygen permeation rates, demonstrate the promise of the material for high-purity oxygen separation below $700{ }^{\circ} \mathrm{C} .544$

Figure 36

Although there has been limited wholesale use of nanomaterials for full working SOFCs ${ }^{426}$ and gas membrane reactions to date (particularly using continuous hydrothermal synthesis), the authors believe that this subject will be increasingly of interest. Future work might focus on formulating the nanomaterials (made via CHFS) by perhaps using the SPS technique to construct a whole-cell fabrication and to assess the redox tolerance and general electrochemical performance for SOFC operation. By taking the advantages of continuous hydrothermal synthesis in producing evenly distributed (or intimately mixed) hybrid oxides and SPS (in rapidly sintering and in situ reducing the metals), it could lead to advanced nano-structured SOFC layers or entire stacks with improved electrochemical performance.

\subsection{Thermoelectrics and Energy Scavenging}


The conversion of thermal energy to electricity is known as thermoelectric (TE) conversion. The TE effect can be used for both electronic refrigeration and power generation. When a temperature gradient $(\Delta T)$ is applied to a TE couple consisting of n-type (electron-transporting) and p-type (hole-transporting) elements, any mobile charge carriers at the hotter end can diffuse to the cold end, producing a potential $(\Delta V)$. This characteristic is known as the Seebeck effect (where $\alpha=\Delta V / \Delta T$, the Seebeck coefficient).

The efficiency of TE devices is strongly associated with the dimensionless figure of merit $Z T$, which is equal to $\left(\alpha^{2} \sigma / \kappa\right) T$, where $\sigma, \kappa$ and $T$ are the electrical resistivity, thermal conductivity and temperature (Kelvin), respectively. $Z T$ values of near to 3, are required to allow TE energy converters to compete on efficiency with mechanical power generation and active refrigeration. The challenge is to tailor the interconnected physical parameters $\alpha, \sigma$ and $\mathrm{k}$ for a crystalline system. Nanocomposites provide a chance to disconnect the linkage between thermal and electrical transport by introducing scattering mechanisms for ZT enhancement.

There are no reports of thermoelectrics being made via continuous hydrothermal syntheses and evaluated in devices to the best of our knowledge, however, there is at least one report of continuous hydrothermal synthesis of $\mathrm{Bi}_{2} \mathrm{Te}_{3}$ nanoparticles using glucose as reductant (when alloyed with $\mathrm{Sb}$ or Se this material has been known to form an efficient thermoelectric material). ${ }^{123}$ This work showed that the manufacture of such materials is highly challenging because of the requirement for simultaneous conversion from precursors as well as the reduction of the metals. Thus, this report on bismuth telluride and the handful of metal sulfides ${ }^{115,} 122,370$ that have been reported, suggests that the synthesis of such chalcogenides for thermoelectrics should be viable if suitable precursors can be used and suitable devices can be made and tested. Therefore, the authors believe that there is sufficient scope in this area to use continuous Hydrothermal synthesis methods on the manufacture of polycrystalline thermoelectric devices (because the presence of grain boundaries is considered beneficial for phonon scattering), e.g. from metal chalcogenides of $\mathrm{S}$, Se and Te using metals such as $\mathrm{Sn}, \mathrm{Cu}, \mathrm{Bi}$, etc.

Other energy scavenging applications of nanoparticels include the use of piezoelectric nanoparticles for energy scavenging devices in which energy is obtained from movement. 406 The pioneering research of the Wang group is highlighted in this area as they have pioneered the use of nanomaterials in devices for such energy scavenging. ${ }^{406} \mathrm{~A}$ number of piezoelectric candidate materials that could be envisaged for such applications have been made by CHFS methods but not tested to date including lead free piezoelectric ceramics ${ }^{30,291,545}$ or indeed $\mathrm{ZnO}$ based nanowires 
amongst others. Some of these materials are mentioned in other sections elsewhere in this review, for example, ZnO has been made and tested for UV attenuating properties.

\subsection{Dye Sensitized or Other Solar Cells}

NPs such as those obtainable via continuous hydrothermal methods, could play a role in solar energy harvesting applications such as dye-sensitized solar cells and more recently, perovskite solar cells (the latter is certainly an area, which is leading to rapid improvement in efficiencies). The most obvious application of continuous hydrothermal nanomaterials is to act as a high surface are mesoporous support for whatever solar absorbers are used. Typically in solar cells, batch hydrothermal titania is used and has a surface area of less than $100 \mathrm{~m}^{2} \mathrm{~g}^{-1}$, which compares to continuous hydrothermal-made titania (which is often doped) that is typically closer to $280 \mathrm{~m}^{2} \mathrm{~g}^{-1} .260,262$ The key point for such applications is also the tight size control that continuous hydrothermal processes can offer (with scalability).

Dye sensitized solar cells (DSSCs) have attracted extensive interest for solar energy harvesting due to their low cost and simple design and ability to be manufactured using scalable roll to roll processes. ${ }^{546}$ A typical DSSC is composed of a working electrode usually made from a mesoporous $\mathrm{TiO}_{2}$ (coated with dye molecules on a conductive transparent oxide (which is usually fluorine doped Sn oxide, FTO), a counter electrode (usually a Pt on a conductive oxide) and a redox electrolyte such as iodine. The photoexcited electrons which are generated in the dye molecules, are injected into the conduction band of $\mathrm{TiO}_{2}$ (upon illumination), and then are transferred into the conductive oxides, whereupon the electron travels through a circuit and back to the counter electrode, to be returned to the dye via the electrolyte. ${ }^{546}$ The interfaces in DSSCs play a significant role for unidirectional charge flow; charge recombination at the interface of the conductive coating (which supports the porous titania) and the electrolyte cannot be neglected because the porous structure of $\mathrm{TiO}_{2}$ cannot completely inhibit recombination from percolation of charge between the electrolyte and FTO (which is supporting the mesoporous titania). Hence, compact and thin blocking layers between FTO and the photo-anode are used to reduce leakage and increase overall efficiency. In this regard, $\mathrm{Li}$ et al. ${ }^{547}$ used electrophoretic deposition to coat a CHFS-made $\mathrm{TiO}_{2}$ blocking layer on a substrate that was then used in dye-sensitized solar cells (DSSCs). There was an improvement in efficiency from 13.1 to $15.0 \%$, when the layer was added. Notably, the titania was made on a scaled up continuous Hydrothermal synthesis pilot plant at a rate of $380 \mathrm{~g} \cdot \mathrm{h}^{-1} .547$ 
There are also opportunities to use nanomaterials in DSSCs or other types of solar cells, e.g. such as more sustainable transparent conducting oxide (TCO) for printed current collectors made from CHFS NPs (see Section 6.1). ${ }^{121,379,384,385}$ Instead of using dyes to absorb solar energy, some designs of solar cells use inorganic materials such as $\mathrm{Cd}$ based compounds or quantum dots..$^{54^{8}}$ Whilst metal sulfides have been made via continuous hydrothermal routes, ${ }^{115,}{ }^{122,} 370$ the authors are not aware if CHFS-made chalcogenides have been developed for solar cells to date.

\section{ENERGY STORAGE APPLICATIONS}

\subsection{Nanomaterials for Energy Storage}

This section will largely focus on the merits of using very small NPs from continuous hydrothermal flow reactors in the field of secondary (rechargeable) batteries and supercapacitors for energy storage. As we can see from Figure 37, supercapacitors are good for high power applications, whilst batteries offer high energy. It is also possible now to manufacture hybrid devices, which use nanoceramics to offer both high power and high energy, sometimes simultaneously.

Figure 37

The current methods of synthesizing Li and Na-ion battery materials, are dominated by spray pyrolysis and batch solid-state heat and grinding methods, and even some batch hydrothermal synthesis. ${ }^{549}$ However, such methods may offer poor reproducibility or lack of control over particle attributes, e.g. being able to make nano-sized particles with specific properties, which may be useful in battery applications. As we will see herein, CHFS methods offer potential benefits for the manufacture of both anode and cathodes in Li-ion batteries and indeed in the case of the Hanwha Corporation in Korea, the process was developed to a larger scale, reportedly with the potential to make hundreds of tonnes pa for olivine cathode materials. 550

Continued interest in high performance secondary Li-ion batteries, has driven the development of new electrode materials and their synthesis techniques, often targeting the scalable production of high quality nanoceramics $(<100$ $\mathrm{nm}$ in diameter) without loss of particle attributes and in cell performance (see later in this section and also in section 10.7 on scale-up). 
Firstly, we shall address the question of what is a Li-ion battery and what are its requirements? A general lithiumion battery consists of an anode and cathode, separated with a separator soaked with a lithium-ion salt containing electrolyte. Upon discharging of the secondary Li-ion battery, Li-ions from the anode are deintercalated and then pass across the separator to the cathode, accompanied by an electron in an external circuit. Upon charging, the reverse happens, i.e. the Li-ions are deintercalated from the cathode and travel via the electrolyte to be intercalated into the anode material (with an electron being accepted by the anode via the charger). This requires the anode and cathode active materials to be in ohmic contact with the current collectors and so active ceramic anodes and cathodes for academic research, are normally printed with for example, a weight ratio of 80:10:10 of active : conductive agent (such as carbon black, Super P):binder (such as in polyvinylidene fluoride, PVDF). Electrolytes for Li-ion batteries typically include $\mathrm{LiPF}_{6}$ in 3:7 wt\% ethylene carbonate / ethyl methyl carbonate. For those wishing to know more on this topic, the UCL authors have provided two relevant videos showing (i) the process of making a printed electrode from a nanopowder ${ }^{551}$ and (ii) assembly of a Li-ion coin cell using the printed electrode. $55^{2}$

Before discussing each type of battery electrode or material choice, it is useful to understand that much of the requirements for battery materials can be understood in terms of the power and energy densities (either gravimetric or volumetric possibly). High power applications tend to mean that a large amount of energy is delivered in a short space of time, whilst high energy is concerned with the total capacity to store energy, which in the case of a laptop will be delivered over a longer time (see Ragone plot later). Nano-sizing battery electrode materials can provide benefits for high power and sometimes for high energy.

Many of the candidate cathode and anode materials actually possess low ionic and electronic conductivities. This can be partly overcome by doping them and / or nano-sizing such materials and covering them with a thin layer or conducting carbons or formulating them with various conducting phases in a printed electrode. The effect of this can be dramatic, because of the significant increase in electronic conductivity (including the possibility for enhanced electron tunneling) and the large surface to volume ratio increase for sub $50 \mathrm{~nm}$ particles, for which the latter, allows more Li ions to be stored nearer to the particle surface. Having Li ions near the surface can benefit one-dimensional Li-ion intercalation materials, which are limited as they only have intercalation and deintercalation channels along one crystallographic direction.

The authors and others have also shown that nano-sized electrode materials also show very different cyclic voltammograms (see current versus potential plots in figure 38 ). This data suggests that well defined intercalation 
charge storage events in bulk materials, can switch to less well defined pseudocapacitive surface charge storage mechanisms in NPs (similar mechanisms that are often seen in supercapacitors). Thus, NPs are ideal for supercapacitor applications as well as high power batteries (as shall be seen later). In a similar way, for a bulk materials, whilst the intercalation / deintercalation of Li-ions occurs at a well-defined potentials, nanomaterials surfaces can support defect sites, which are known to increase Li charge storage. As shown in figure 38 , the potential vs capacity plots show that in a bulk electrode the potential is at a well-defined plateau, which is different to a nanomaterial displaying pseudocapacitive charge storage.

Figure 38

\subsection{Li -ion Battery Cathodes}

Cathode materials are generally required as one of the electrodes in Li-ion batteries for mobile phones, 553 laptops 554 and many other electronic types of equipment $555-557$. These tend to operate at higher voltage ranges that the corresponding anodes and due to generally lower capacities compared to anodes, there is a great deal of interest in extending the specific capacities of cathode materials. ${ }^{20,} 283,558-562$

The vast majority of research in continuous hydrothermal cathodes has been on metal oxides and phosphates. Iron sulfides have been made via continuous hydrothermal, ${ }^{370}$ but not really tested to any extent for potential applications in Li-ion battery cathodes to date. In order to increase the energy densities, higher voltage cathodes are required. However, this has demanded the development of new electrolytes, which are stable at higher voltages, so this brings additional research effort and potentially more cost. Overall, the specific capacity of a cell is related to its operating voltage window and therefore, in the future, researchers seek to increase the capacity of cells via improvements in materials or indeed new types of cells such as Li air cells (see figure 39). The latter types of cells may well require NP catalysts, which is an area in which continuous hydrothermal may prove useful in the next few years.

Figure 39

Since good electronic conductivity and stable lithium ion diffusion, are important factors in achieving highperformance in rechargeable lithium batteries, highly crystalline materials with high surface area are thus, of inter- 
est. ${ }^{563}$ Many researchers have tried various methods to achieve this goal, however, most of them use solid-state routes that involve high temperatures ${ }^{564}$, or routes that need complex steps ${ }^{565-566}$, resulting in large particles and long processing time. However, some alternative methods, such as sol-gel, hydrothermal synthesis ${ }^{549}$ and ionexchange techniques, which are carried out at relatively milder temperatures are generally associated with relatively low crystallinity ${ }^{19,} 567$.

Li-Co Oxide (LCO) Based Cathodes. Supercritical water synthesis, has been shown to be one of the best methods in producing crystalline and uniform NPs, which are of interest as cathodes for rechargeable batteries. ${ }^{197,549}$ Indeed, Adschiri and co-workers ${ }^{20}$ manufactured $\mathrm{LiCoO}_{2}$ crystals continuously in a hydrothermal flow system. $0.02 \mathrm{M}$ $\mathrm{Co}\left(\mathrm{NO}_{3}\right)_{2}$ and $0.4 \mathrm{M} \mathrm{LiOH}$ aqueous solutions were used as starting materials, and $0.07 \mathrm{M} \mathrm{H}_{2} \mathrm{O}_{2}$ was also introduced into reactor as an oxygen source (for oxidizing $\mathrm{Co}^{2+}$ to $\mathrm{Co}^{3+}$ ). In their report, phase pure rock salt $\mathrm{LiCoO}_{2}$ could be synthesized at supercritical conditions, whereas at 300 or $350^{\circ} \mathrm{C}_{1} \mathrm{CO}_{3} \mathrm{O}_{4}$ spinel was produced. More effective oxidation of $\mathrm{Co}^{2+}$ happened at $400{ }^{\circ} \mathrm{C}$, which was probably due to the formation of the reaction atmosphere of the solution and oxygen gas. Particle size of crystals was in the range of ca. 0.5 to $1.0 \mu \mathrm{m}$, and the electrochemical properties of the $\mathrm{LiCoO}_{2}$ crystals evaluated by a constant current discharge and charge tests, suggested high stability of the material prepared by this method.

Kanamura et al..$^{568}$ produced $\mathrm{LiCoO}_{2}$ by both supercritical water synthesis and solid state reaction methods, and found out material obtained in continuous hydrothermal flow system, had much smaller particle size than one made by a solid-state reaction. Intercalation and deintercalation of $\mathrm{Li}$-ions into and from $\mathrm{LiCoO}_{2}$ particles, were conducted with good reversibility. More recently, Shin and co-workers reported the continuous hydrothermal synthesis of $\mathrm{LiCoO}_{2}$ (nanoplates) via the reaction of cobalt nitrate, $\mathrm{LiOH}$, and $\mathrm{H}_{2} \mathrm{O}_{2} .{ }^{569}$ The flow experiments were carried out in both subcritical and supercritical water, at temperatures in the range 300 to $411{ }^{\circ} \mathrm{C}$, with residence times typically $<1$ min. Under supercritical conditions, the variations in temperature and residence time did not have a significant bearing on particle size distribution, the average crystallite size or on the morphology. ${ }^{69}$ Importantly, an excess of hydroxide and peroxide were required to enable the materials to be made phase pure.

In a report by Anikeev, ${ }^{383}$ a number of $\mathrm{LiMO}_{n}$ compounds such as $\mathrm{LiCoO}_{2}$ and $\mathrm{LiNiO}_{2}$ (as well as $\mathrm{LiZnO}_{2}$, and $\mathrm{LiC}$ $\mathrm{uO}_{2}$ ) were made in a tubular flow reactor. Unfortunately, as in the case of many of the reports where these materials were made in flow, they were not however actually tested as cathodes for Li-ion battery materials. 
$\mathrm{LiMn}_{2} \mathrm{O}_{4}$ (LMO) Type Cathodes. Similarly, another Li-ion battery cathode material is the spinel $\mathrm{LiMn}_{2} \mathrm{O}_{4}$. $\mathrm{LiMn}_{2} \mathrm{O}_{4}$ was prepared using a hydrothermal flow process in supercritical water at $400{ }^{\circ} \mathrm{C}$ and $30 \mathrm{MPa} .5^{61} \mathrm{LiOH}(0.05$ M) and $\mathrm{Mn}\left(\mathrm{NO}_{3}\right)_{2}(0.05 \mathrm{M})$ salt solutions were used as starting precursors and $\mathrm{H}_{2} \mathrm{O}_{2}$ was added with various molar ratios in order to control an oxidation state of $\mathrm{Mn}$ ions in as-prepared samples. The report indicated that the addition of $\mathrm{H}_{2} \mathrm{O}_{2}$ into supercritical water was effective in preparing spinel $\mathrm{LiMn}_{2} \mathrm{O}_{4}$ in a single phase. The prepared $\mathrm{LiMn}_{2} \mathrm{O}_{4}$ had a relatively small crystallite size $(<0.5 \mu \mathrm{m})$, but relatively poor performance as a cathode material (only 85 mAh. $\mathrm{g}^{-1}$ under cycling at $0.1 \mathrm{C}$ ), which was lower than the theoretical capacity $\sim 148 \mathrm{mAh} . \mathrm{g}^{-1}$. Note: a $1 \mathrm{C}$ represents a charge or discharge in 60 mins; to calculate other $C$ rates, the time is divided by 60 mins, i.e. a fast charge of $30 \mathrm{C}=$ $60 / 30=2$ minutes. Additional heat-treatment was performed on as-precipitated $\mathrm{LiMn}_{2} \mathrm{O}_{4}$ and the sample heated to $800^{\circ} \mathrm{C}$ had improved discharge capacity and cycle stability, with a capacity of $125 \mathrm{mAh} . \mathrm{g}^{-1}$ (at $0.1 \mathrm{C}$ after $60 \mathrm{cycles}$ )..$^{61}$ The researchers concluded that $\mathrm{LiMn}_{2} \mathrm{O}_{4}$ obtained by hydrothermal synthesis had a much better electrochemical performance than that made via the conventional solid-state method, generally because the former contained an excess $\mathrm{Li}$ and the average oxidation state of $\mathrm{Mn}$ was higher. ${ }^{561} \quad \mathrm{LiMn}_{2} \mathrm{O}_{4}$ particles were also made via a continuous hydrothermal process by Lee and Ham., but were not evaluated as cathodes. 570 Different phases such as $\mathrm{LiMn}_{2} \mathrm{O}_{4 \prime}$ $\mathrm{Mn}_{2} \mathrm{O}_{3}$ and $\mathrm{MnO}_{2}$ were obtained depending on process conditions and reactant compositions. Selective synthesis of phase pure $\mathrm{LiMn}_{2} \mathrm{O}_{4}$ was dependent of the amount of $\mathrm{OH}^{-}$ions, but not necessarily the Li/Mn ratio or lithium nitrate amount. Particle size of $\mathrm{LiMn}_{2} \mathrm{O}_{4}$ was ca. $48 \mathrm{~nm}$ (Figure 40). The authors concluded that the amount of OH-ions, had the most significant effect on the reaction pathways and the size and structure of particles obtained. 570

\section{Figure 40}

In order to understand how lithiated-phases can form under hydrothermal conditions, in situ XRD batch hydrothermal studies conducted over very short timescales (seconds), can often give useful insights. A study by Iversen and colleagues ${ }^{178}$ on the hydrothermal formation of $\mathrm{LiMn}_{2} \mathrm{O}_{4}(\mathrm{LMO})$ nano-crystallites, revealed that the reaction progresses in steps, that can be sumarised as $\mathrm{KMnO}_{4}$ to disordered $\delta-\mathrm{MnO}_{2}$, to disordered $\delta-\mathrm{MnO}_{2}$ to $\mathrm{LiMn}_{2} \mathrm{O}_{4}$ to $\gamma^{-}$ $\mathrm{Mn}_{2} \mathrm{O}_{3}$ to $\mathrm{Mn}_{3} \mathrm{O}_{4}$. Thus, LMO is formed as an intermediate. The phase purity of LMO was therefore dependent on reaction time / temperature, giving phase pure LMO after 150 to 210 seconds at $220^{\circ} \mathrm{C}$ ( 45 to 140 seconds at $260^{\circ} \mathrm{C}$ ). 
Furthermore, in situ measurements suggested that LMO first appeared to form as thin platelets (size range 3 to 13 $\mathrm{nm}$ ) which progressed by growing faster along the [111] direction to give rods. ${ }^{178}$

Lithium Iron Phosphate (Olivine) Based Cathodes. Perhaps the most promising new commercial candidates for Liion battery cathodes are based on lithium iron phosphate, LFP $\left(\mathrm{LiFePO}_{4}\right)$, which were discovered by Goodenough et al. ${ }^{571}$ Such cathodes are considered more sustainable and safer alternatives to commercial Li-ion battery cathodes based on Co and Mn. ${ }^{572}$ The literature clearly shows that the synthesis route requires slightly reducing synthesis conditions and synthetic approach, is a key factor that determines cathode composition/properties and performance. ${ }^{549}$ For example, solid-state synthesis can give rise thermodynamic products, which may be inhomogeneous (due to mass transfer limitations of the processing) i.e. resulting in mixed phases. However, sometimes, a secondary impurity phase alongside the olivine structure can benefit the cathode performance. For example, vanadium phosphate impurity phases can sometimes be co-synthesized along with V-doped LFP and enhance conductivity. ${ }^{573}$ Thus, whilst solid-state routes are scalable to some extent and offer moderate performance at this scale, they are not suited to make nano-sized particles (which are better for high power materials).

A number of high performing battery nanomaterials in the literature, often make samples at very small scales or using significant amounts of surfactants and chemicals. However, it is often very unlikely that this performance can be readily replicated at scale with reproducibility and reasonable cost. As shall be seen below, many high performance cathode materials have been synthesized via simple continuous hydrothermal processes, $549,562,574-577$ and also then evaluated as cathodes at large-scale (capacity potentially up to 1000 tpa if fully operational) by the Hanwha Corporation of Korea (see Figure 53 later on in section in scale-up). .50

A number of cathode Li ion battery materials have been made via CHFS to date, and in some cases the nanomaterials have been tested in half-cells versus lithium chip. This often shows excellent properties when tested (due to the large excess of $\mathrm{Li}$ in the counter electrode), however, in order to go towards commercial applications, such materials need to be tested in full cells versus a relevant anode. To our knowledge, there has only been one report in the academic literature in which a full cell has been made using at least a nanomaterial made via CHFS..$^{60}$ In that report, nano-sized vanadium-doped LFP (V-LFP with 5 at\% dopant) made via CHFS was cycled against an inexpensive micron-sized metallurgical grade Si-containing anode. ${ }^{560}$ At the time of writing, there is still no report in the academic Li-ion battery literature in which both active components have been made via CHFS. 
In the discussion below, we will now explain why LFP based cathodes benefit from being made using continuous hydrothermal synthesis methods and how metastable forms can be manufactured. Its first important to understand the structure and properties of LFP and this will explain why it can benefit from nano-sizing and doping. LFP has a Pmnb space group and the volume of the unlithiated unit cell (272.4 $\left.\AA^{3}\right)$ is less than the lithiated LFP (291.4 $\left.\AA^{3}\right)$. The structure of LFP (olivine structure) is made from corner-shared $\mathrm{FeO}_{6}$ octahedral units and phosphate tetrahedra, which are linked creating channels between them for the $\mathrm{Li}$ ions to sit in. The $\mathrm{FeO}_{6}$ octahedra share $\mathrm{O}$ atoms with the corners of the $\mathrm{PO}_{4}$ tetrahedra, resulting in a three-dimensional zigzag framework containing intercalated $\mathrm{Li}^{+}$ions ( $\mathrm{LiO}_{6}$ groups) forming a linear chain of edge-shared octahedra which are parallel to the $b$ axis. Despite the fact that undoped bulk LFP, can be made scalably via solid state and other methods, it is potentially unsuitable as a cathode material because it does not possess a sufficient balance of high electronic conductivity and high ionic diffusivity, (reported to be in the range ca. $10^{-9}$ to $10^{-8} \mathrm{~S} \cdot \mathrm{cm}^{-1}$ and $10^{-17}$ to $10^{-12} \mathrm{~cm}^{2} \mathrm{~s}^{-1}$, respectively). ${ }^{578-580}$

Batch hydrothermal reactions for the synthesis of LFP micro and nanoparticles, have been reported such as by Teja et al., , $^{81-582}$ who upon testing the heat-treated products, achieved specific capacities in half cells of ca. 140 $\mathrm{mAh} \cdot \mathrm{g}^{-1}$ at only $0.1 \mathrm{C}$ (and closer to $100 \mathrm{mAh} \cdot \mathrm{g}^{-1}$ at $1 \mathrm{C}$ )..$^{582}$ Clearly, flow reactors offer much more promise to be able to develop very much smaller particles that should display high capacities at higher power situations as will be seen later in this section. It should be noted that even if such materials are made via CHFS, they generally still require a post synthesis heat-treatment (typically $600{ }^{\circ} \mathrm{C}$ in a reducing atmosphere) to overcome the anti-site defects, otherwise the capacity is often reduced considerably. This is in contrast to many of the anode materials for Li-ion batteries, which used directly from continuous hydrothermal processes after drying. ${ }^{29,}$, $283,583-584$

Continuous hydrothermal synthesis of $\mathrm{LiFePO}_{4}$ was reported by $\mathrm{Xu}$ et al., where a precursor ratio of 1:1:3 $\mathrm{FeSO}_{4}: \mathrm{H}_{3} \mathrm{PO}_{4}: \mathrm{LiOH}$ produced phase-pure $\mathrm{LiFePO}_{4}$ for both subcritical and supercritical water feeds.577 The use of supercritical water resulted in an increase in particle size compared to the subcritical case (made at a scale of ca. 0.3 g.h $\mathrm{h}^{-1}$ assuming $100 \%$ yield). In contrast, Aimable et al. were able to synthesize LiFePO ${ }_{4}$ with a residence time of $12 \mathrm{~s}$ by using a Fe:P:Li ratio of 1:1:3.75. ${ }^{562}$ However, of the materials that were tested, the best high-rate performing $\mathrm{LiFePO}_{4}$ had a modest capacity (105 mAh.g-1 at $\left.1 \mathrm{C}\right)$. Kim and co-workers reported a CHFS study to make nano-sized lithium iron phosphate in the pressure range 25 to $30 \mathrm{MPa}$ and at $400{ }^{\circ} \mathrm{C}$. The discharge capacity of the LiFePO${ }_{4}$ (which was not phase pure as made due to oxide impurities) delivered only $90 \mathrm{mAh} \cdot \mathrm{g}^{-1}$ for up to ten cycles, however, with a carbon coating, the discharge capacity of $\mathrm{C}-\mathrm{LiFePO}_{4}$ was as high as $141 \mathrm{mAh} . \mathrm{g}^{-1}$, which corresponds to $84 \%$ of 
the theoretical capacity. ${ }^{85}$ It should be remembered that these materials were cycled at only a $0.1 \mathrm{C}$ rate, which was relatively slow and this would be therefore be expected to get near to the theoretical value. In a second report, Kim and co-workers reported the continuous hydrothermal synthesis particles of LFP in the size range 200 to $800 \mathrm{~nm}$, with BET surface areas in the range 6.3 to $15.9 \mathrm{~m}^{2} \mathrm{~g}^{-1}$. LiFePO ${ }_{4}$ synthesized in supercritical water exhibited a discharge capacity in the range 70 to $80 \mathrm{mAh} . \mathrm{g}^{-1}$ at $0.1 \mathrm{C}$ after 30 cycles. A smaller capacity fade from 135 to $125 \mathrm{mAh} . \mathrm{g}^{-1}$ was then observed during the 30 cycles in carbon-coated $\mathrm{LiFePO}_{4}$ synthesized using supercritical water. ${ }^{575}$ In a third report by the Kim and co-workers,574 carbon content, carbon structure, morphology, electronic conductivity, and electrochemical performance of the carbon-coated $\mathrm{LiFePO}_{4}\left(\mathrm{C}-\mathrm{LiFePO}_{4}\right)$, were studied as a function of the process conditions, i.e. sucrose concentration, post synthesis heat-treatment temperature and time. The particles produced using supercritical water had sizes in the range $400-1000 \mathrm{~nm}$, with a BET surface area of $7.3 \mathrm{~m}^{2} \mathrm{~g}^{-1}$ and lower degree of particle aggregation compared with those produced via solid-state synthesis. The authors suggested that the differences in particle attributes and performance were due to the differences in the uniformity of the carbon coating, carbon structure, and electronic conductivity. 574 A less uniform carbon layer coating was suggested for the hydrothermal flow process, which gave a capacity of $135 \mathrm{mAh} \cdot \mathrm{g}^{-1}$ at $0.1 \mathrm{C}$. At a relatively high current of $1.7 \mathrm{~A} \cdot \mathrm{g}^{-1}$ (10C), the carbon coated LFP particles produced via the solid state and continuous hydrothermal method were 55 and $52 \%$, respectively, of the theoretical values ( $6 \mathrm{wt} \%$ carbon). .574

A further way to improve the performance of LFP in Li-ion batteries is using doping of metal ions to enhance conductivity of the active phase, which also has the effect of subtly distorting the olivine lattice in such a way as to enhance Li-ion transport. If the dopant is electrochemically active in a wider potential range vs $\mathrm{Li}^{\prime} \mathrm{Li}^{+}$that that normally used for LFP, it can also lead to increase energy storage. For example, Mn-doping in the LFP can give a higher theoretical energy, but not necessarily higher power capability. ${ }^{586}$ The authors recently reported the synthesis of Vdoped $\mathrm{LiFePO}_{4} / \mathrm{C}$ (and the undoped analogue) on a pilot-plant scale CHFS process. ${ }^{866}$ Samples containing vanadium at $2.5,5,10$, and 20 at\% (with respect to $\mathrm{Fe}$ ) were carbon-coated in process and the dried powders were all heattreated to graphitize the carbon coatings and remove anti-site defects (Figure 41). The residence time of only $6.5 \mathrm{~s}$ in the continuous reactor was sufficient to obtain phase pure materials. From this report the sample with composition $\mathrm{Fe}_{0.95} \mathrm{~V}_{0.05} \mathrm{PO}_{41}$ achieved a reversible specific discharge capacity of $119 \mathrm{mAh} . \mathrm{g}^{-1}$ at a high discharge rate of $1.5 \mathrm{~A} . \mathrm{g}^{-1}$ (this rate is ca. 9 C, i.e. $60 / 9=6.67$ minutes charge and discharge). ${ }^{586}$ This was comparable to the best performances achieved in the academic literature for similar materials. Moreover, when it is considered that the samples were 
made on a pilot-plant production scale (6 kg per day), the result is even more remarkable. In the same report, it was found that experimental and computational techniques, ${ }^{586}$ were consistent with the assertion that vanadium doping can occur on both Fe and $\mathrm{P}$ sites within the structure at a low level. However, the topic of $\mathrm{V}$ substitution in olivines is contentious and, therefore, more definitive characterization will be required in future to ascertain if the $V$ can indeed replace some of the $P$ in the LFP structure.

Figure 41

High power nano-sized phase pure Nb-doped $\mathrm{LiFePO}_{4}$ (LFP) NP cathodes (carbon-coated), were recently developed using the using a pilot-scale CHFS process (rate of $\left.0.25 \mathrm{~kg} \cdot \mathrm{h}^{-1}\right) .5^{8}$ Six Nb-dopant levels in the range 0.01 and 2 at\%, were doped into the olivine, which were then heat-treated. Electrochemical testing showed the performance of the doped materials improved with increasing dopant concentration up to a maximum at 1.0 at $\%$, which gave a specific capacity of $110 \mathrm{mAh} \cdot \mathrm{g}^{-1}$ at $10 \mathrm{C} .5^{58}$ It was suggested that the $\mathrm{Nb}$ in the sample not only distorted the lattice (it would be expected to replace the Fe in octahedral sites), which aided Li-ion transport, but it also possibly enhanced conductivity of the samples. ${ }^{558}$

Mn doping into LFP (samples known as LMFP) has also been achieved using continuous Hydrothermal synthesis at a production rate of $1 / 4 \mathrm{~kg} \cdot \mathrm{h}^{-1} .559$ For the complete replacement of Mn for Fe in LFP (known as LMP), the LMP has an increased theoretical energy density in comparison to LFP (697 vs. 586 Wh. $\left.\mathrm{kg}^{-1}\right)^{587}$ however, LMP displays lower electronic conductivity than LFP $\left(10^{-10} \mathrm{~S} \cdot \mathrm{cm}^{-1}\right.$ compared to the range ca. $10^{-7}$ to $\left.10^{-10} \mathrm{~S} \cdot \mathrm{cm}^{-1}\right) \cdot{ }^{588}$ Therefore, these materials are more important for low power applications where energy density is more important. In the report from some of the UCL authors, phase pure, nano-sized Mn-doped $\mathrm{LiFePO}_{4} \mathrm{NPs}$ (carbon-coated), were made via the pilot scale ${ }^{271}$ continuous Hydrothermal synthesis process.559 Four dopant levels of $\mathrm{Mn}$ in LFP were made at $11,21,57$, and 81 at\%. Raman studies confirmed that the particles were substantially coated with amorphous carbon, which became more graphitic and flatter after a further heat-treatment. Electrochemical testing suggested that $\mathrm{LiFe}_{0.43} \mathrm{Mn}_{0.57} \mathrm{PO}_{4}$ was an ideal material for low current applications, with a specific capacity 161 mAh.g ${ }^{-1}$ (energy

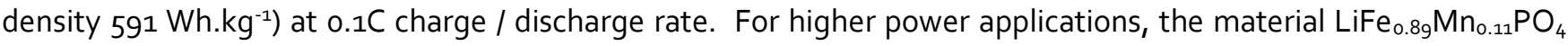
was found to be the best with a specific capacity of $108 \mathrm{mAh} . \mathrm{g}^{-1}$ (calculated energy density of $301 \mathrm{Wh}^{\mathrm{kg}} \mathrm{k}^{-1}$ ) at an impressive ${ }_{5} \mathrm{C}$ rate. ${ }^{559}$ Figure $42 \mathrm{a}$ shows the cycling performance of the Fe-doped LMP family of cathode materials at 
different $\mathrm{C}$ rates. Whilst, Figure $42 \mathrm{~b}$ shows the $\mathrm{LiMn}_{1-x-y} \mathrm{Fe}_{x} \mathrm{~V}_{y} \mathrm{PO}_{4 \prime}$ area under investigation highlighted with a green triangle, which apply to the heat-maps at C-rates of C/2 (Figure $42 \mathrm{C}$ ) and ${ }_{5} \mathrm{C}$ (Figure $42 \mathrm{~d}$ ).

Figure 42

Other Potential Li lon Cathode Materials. Continuous hydrothermal processes will no doubt have a number of future opportunities for Li-ion cathodes. Indeed in many instances, the materials have possessed high specific capacities or high energies in a Li-ion coin cell. 549 There are of course a number of other cathode materials, which would still warrant some attention due to their potential high capacities if the chemistries were amenable to continuous hydrothermal flow methods. The benefits of nano-sizing via continuous hydrothermal are explained above in broader terms. These materials include lithium-excess layered oxides with stable reversible capacities of 220 mAh.g. ${ }^{-1}$ with composite electrodes containing sub-micrometer particles of $0.5\left[\mathrm{Li}_{2} \mathrm{MnO}_{3}\right] \cdot 0.5\left[\mathrm{LiMn}_{0.3} \mathrm{Ni}_{0.3} \mathrm{Co}_{0.3}\right] \mathrm{O}_{2}(2.0$ to $4.8 \mathrm{~V}$ working voltage range), ${ }^{589}$ or the high capacity cathodes based on $\mathrm{Li}_{2} \mathrm{MSiO}_{4}$ (Where $\mathrm{M}=\mathrm{Mn}, \mathrm{Fe}, \mathrm{Co}$ etc.), ${ }^{549}$ amongst others. For the latter material, it is highly likely that forcing conditions would be required as well as a suitable silicate source in order to deliver such materials in a phase pure form (as can be achieved in batch hydrothermal supercritical reactions). However, the very large capacity of the ultrathin $\mathrm{Li}_{2} \mathrm{MnSiO}_{4}$ nano-sheets and $\mathrm{Li}_{2} \mathrm{FeSiO}_{4}$ materials made via batch supercritical processing at $400^{\circ} \mathrm{C}$, has a large capacity of over $340 \mathrm{mAh} \cdot \mathrm{g}^{-1}$ (albeit at an elevated temperature of $45 \pm 5^{\circ} \mathrm{C}$ ). However, this high capacity was only achieved after many processing steps, as the process included heating in an autoclave at $400{ }^{\circ} \mathrm{C}$ for $10 \mathrm{~min}$ and then ball-milling with poly $\left(3,4^{-}\right.$ ethylenedioxythiophene) conductive polymer and multiwall carbon nanotubes, followed by mild heat-treatment at $300{ }^{\circ} \mathrm{C}$ for $3 \mathrm{~h}$ under $\mathrm{Ar}$ atmosphere. Using a flow process, it may be possible to co-precipitate various conductive with the active materials themselves, thereby reducing the extra number of processing steps. In the case of lithiumexcess layered oxide particles of $0.5\left[\mathrm{Li}_{2} \mathrm{MnO}_{3}\right] \cdot 0.5\left[\mathrm{LiMn}_{0.3} \mathrm{Ni}_{0.3} \mathrm{Co}_{0.3}\right] \mathrm{O}_{2,}$, it is unlikely that CHFS reactors can directly make the higher capacity materials of this type. However an initial precipitate of the oxides/hydroxides followed by a high temperature heat-treatment / lithiation to yield the thermodynamic product mixtures of phases, should make these high temperature mixtures possible (at around $900{ }^{\circ} \mathrm{C}$ in the presence of excess Li source no doubt)..$^{89}$ Other less well-known cathodes that might be interesting to make via CHFS include lithium Nickel Cobalt Aluminum oxide (NCA), which, potentially offers high specific energy and reasonably good specific power and a long life span.590 
Other potential cathode materials, which might be accessible via CHFS, include those based on metal sulfides. For example, lithium sulfur batteries have the potential to reversibly store considerable electrical energy at low cost. ${ }^{591}$ Whether or not Li-S batteries will be able to fulfil this potential depends on simultaneously solving many aspects of its underlying conversion chemistry which is complex and the use of additives to improve it. 592

Li-air cell is a challenging type of energy storage and conversion device. Its successful commercialization highly depends on the timely development of key components (including nano-catalysts, which will be a key part of the air electrode). ${ }^{593}$ Within the next 30 years or longer, cathodes co-catalyst components for Li air batteries may also be required to be nano-sized and CHFS will be an ideal way to make such materials. Looking nearer to the future, there are exciting possibilities of multivalent cathodes (e.g. Al or Mg-ion batteries) such as those based around spinel structures ${ }^{594}$ possessing large pores. If magnesium-ion (or Al or other ion) batteries can be realized in the next decade or so, they have a high theoretical volumetric capacity due to doubly charged $\mathrm{Mg}^{2+}\left(3832 \mathrm{mAh} \mathrm{cm}^{-3}\right.$ for $\mathrm{Mg}$ vs. $2062 \mathrm{mAh} . \mathrm{cm}^{-3}$ for Li and $1136 \mathrm{mAh} \cdot \mathrm{cm}^{-3}$ for $\mathrm{Na}$ ). Other benefits include high safety (dendrite-free Mg deposition), low cost and good sustainability (earth abundant Mg). $595-596$

As continuous hydrothermal processes continue to develop for energy storage, the integration or protection of the active materials in electrodes with carbons of various kinds in process (or indeed inert coatings to protect the actives), should be developed. This could offer more complex, resilient and / or conductive composite NPs, which will perform better under demanding high power conditions. Other areas for development will be to make NP inks for inkjet printing which would facilitate printable or flexible batteries in future. In terms of testing these novel materials, most of the electrochemical cell tests are vs Li chip as the counter electrodes. Thus, in order to verify that these materials indeed have promise, they need to be evaluated against suitable anode materials, some of which are covered below.

Finally, in order for battery materials to reach the marketplace, the scale-up of battery materials brings its own challenges. In order for these materials to be of interest to industry, it will be important to go from the lab scale and small coin cells and test them on yet larger scale cells and evaluating the cathode materials on $\mathrm{Kg} \cdot \mathrm{h}^{-1}$ or higher scales in order to move up the technology readiness levels and prove the technology has potential for industry. Other than the efforts from the Hanhwa Corporation 550 and the abovementioned work by the authors, $32,170,261,586$ this has not been carried out routinely at scales of more than a few $\mathrm{g} \cdot \mathrm{h}^{-1}$ despite the tremendous performance benefits that may accrue if higher surface area battery electrodes can be made direct in process. As we move from petrol engines to- 
wards hybrid engines (and eventually full electric in the future), the market for nano-sized high power cathode (and anode) materials will increase. Therefore, large-scale continuous hydrothermal processes will become increasingly likely.

\subsection{Lithium Ion Battery Anodes}

Different from cathode materials, there are more classes of anode materials including insertion/intercalation, conversion and alloying materials, which are all based on (transition) metal oxides offering a huge number of possible candidates synthesized via CHFS routes. Insertion materials such as (lithium) titanium oxides (e.g. $\mathrm{TiO}_{2}=168$ $\mathrm{mAh} . \mathrm{g}^{-1}$ and $\mathrm{LTO}=175 \mathrm{mAh} \cdot \mathrm{g}^{-1}$ ) offer good sustainability, low cost, low risk to the environment, high cell safety, low capacity loss, high power capability and a very high cycle life due to minimal volume and structural change during cycling. However, they often have a narrower operational potential window, which dramatically decreases specific energy. 597

In 2000, Poizot et al. investigated various metal oxides such as $\mathrm{CoO}$ and $\mathrm{FeO}$ as negative electrode materials and measured very high capacities of $700 \mathrm{mAh} \cdot \mathrm{g}^{-1} .{ }^{598}$ It was shown that during charge, some metal oxides were (fully) converted to the metallic state, which motivated them to be called conversion materials. There are a huge number of possible conversion materials including transition metal oxides ( $\mathrm{Co}, \mathrm{Fe}, \mathrm{Cu}, \mathrm{Ni}, \mathrm{Mn}$ oxides and many more) ${ }^{598}$ and sulfides, which all show high capacities (e.g. $\mathrm{Fe}_{2} \mathrm{O}_{3}=1007 \mathrm{~mA} \cdot \mathrm{h} \mathrm{g}^{-1}$ ) but a huge voltage hysteresis, high irreversible capacity loss and low cycle life due to drastic structural changes within the electrode network. The voltage hysteresis drastically decreases the energy density of the cell (high delithiation voltage) which is a limitation for their commercial development.

Alternative Li-ion anode materials such as alloying materials ( $\mathrm{Si}, \mathrm{Sn}, \mathrm{Sb}, \mathrm{Zn}, \mathrm{Ag}, \mathrm{Al}$ and more) show high theoretical capacities (e.g. $\mathrm{Si}=3579 \mathrm{mAh} \cdot \mathrm{g}^{-1}, \mathrm{Sn}=993 \mathrm{mAh} \cdot \mathrm{g}^{-1}$ and also a lower operating voltages ${ }^{599}$ compared to insertion materials. ${ }^{590}$ The high capacity and the wider operating potential window result in higher energy densities, however, they show large irreversible capacity loss during the initial cycles and high capacity fading (poor cycle life) due to tremendous volume and structural changes in the anodes during each lithiation and delithiation cycle (e.g. $>$ 200+ vol\% change for Sn alloys). ${ }^{600}$

A number of simple transition metal oxides made using continuous hydrothermal process including CuO, $\mathrm{NiO}$, and $\mathrm{MnO}_{2}, \mathrm{Co}_{3} \mathrm{O}_{41} \mathrm{Fe}_{2} \mathrm{O}_{3}$ particles, were evaluated as anodes for Li-ion batteries. ${ }^{85}$ However, the particles 
were rather large (as shown by their low surface areas and from TEM images) and the electrodes generally showed poor cycle stability. ${ }^{585}$ The initial capacities were (in brackets) $\mathrm{CuO}\left(791 \mathrm{mAh} \cdot \mathrm{g}^{-1}\right), \mathrm{NiO}\left(878 \mathrm{mAh} \cdot \mathrm{g}^{-1}\right), \mathrm{MnO}_{2}(1,110$ mAh.g $\left.\mathrm{g}^{-1}\right), \mathrm{Fe}_{2} \mathrm{O}_{3}\left(951 \mathrm{mAh} \cdot \mathrm{g}^{-1}\right)$, and $\mathrm{Co}_{3} \mathrm{O}_{4}\left(1,132 \mathrm{mAh} \cdot \mathrm{g}^{-1}\right)$ and after ten charge and discharge cycles, the capacity decreased in the range 82 to $62 \%$ of the initial values. ${ }^{58}$ It should remembered that these are storing charge from the conversion reaction of the metal oxide with Li-ions and therefore, in the absence of smaller particles or a more sophisticated conductive network in the electrode, the fading on cycling was not surprising.

Titania or Titanate Based Anodes. Lithium titanate, $\mathrm{Li}_{4} \mathrm{Ti}_{5} \mathrm{O}_{12}(\mathrm{LTO})$ spinel phase has attracted interest as a highly stable, high power anode material as it undergoes very little structural changes during the discharge/charge process and possesses high Li-ion mobility. As an anode, it has a stable voltage plateau at $1.5 \mathrm{~V} \mathrm{vs} \mathrm{Li}^{\mathrm{Li}} \mathrm{Li}^{+}$. In the academic published literature, the direct CHFS synthesis of $\mathrm{Li}_{4} \mathrm{Ti}_{5} \mathrm{O}_{12}$ (LTO) was reported using lithium ethoxide and titanium isopropoxide as reactants at near- and supercritical conditions. ${ }^{601}$ The LTO NPs had an average crystallite size of ca. $4.5 \mathrm{~nm}$ with a BET specific surface area of $230 \mathrm{~m}^{2} \mathrm{~g}^{-1}$. The same authors also reported the use of in-situ synchrotron powder X-ray diffraction measurements on annealed LTO nano-crystallites to investigate the structural and microstructural changes in the range from room temperature to $727^{\circ} \mathrm{C}$. The as-prepared NPs showed significant strain, which was reduced by annealing. Electrochemical tests of two samples were carried out for the as-made LTO and a sample annealed at $600^{\circ} \mathrm{C}$, which showed that specific capacity and rate capability was significantly improved for the annealed sample. For the as-prepared material, the first charging cycle at a rate of $0.1 \mathrm{C}$ showed a high irreversible capacity of $220 \mathrm{mAh} . \mathrm{g}^{-1}$. In subsequent cycles, this was reduced to below the theoretical capacity of $175 \mathrm{mAh} . \mathrm{g}^{-1}$. By charging and discharging the as-prepared material at higher rates (up to $20 \mathrm{C}$ for both charge rate and discharge rate), the specific capacity was ca. 80 and $100 \mathrm{mAh} . \mathrm{g}^{-1}$ for charging and discharging, respectively ${ }^{601}$ For $1 \mathrm{C}$ charge / discharge rates, the specific capacity dropped down to ca. $140 \mathrm{mAh} \cdot \mathrm{g}^{-1}$, and then down to $100 \mathrm{mAh} \cdot \mathrm{g}^{-1}$ after a further 85 cycles.

Heat-treated LTO showed superior electrochemical performance compared to the as-prepared LTO and the characteristic plateau at $\sim 1.55 \mathrm{~V}$ vs $\mathrm{Li}^{\mathrm{Li}} \mathrm{i}^{+}$of the former, was flatter and longer. Cycling tests for the heat-treated LTO showed an initial specific capacity of $\sim 160$ mAh.g $\mathrm{g}^{-1}$ with $95 \%$ capacity retention after 50 cycles. ${ }^{601}$ This report was certainly excellent in terms of performance, however, the authors note that the precursors were relatively ex- 
pensive compared to less chemically intensive alternatives and thus, in terms of scale-up potential, this would significantly add to the cost of production.

The sensitivity to reactions conditions in the continuous hydrothermal processes to the successful synthesis of phase pure LTO was underlined by the recent report by Hayashi and co-workers. ${ }^{602}$ They observed that single phase LTO NPs could be synthesized using an initial Li/Ti molar ratios >2 for various titania sols (and Li OH), whereas they were unable to make it from $\mathrm{Ti}\left(\mathrm{SO}_{4}\right)_{2}$ (aq.) solutions, even with initial $\mathrm{Li} / \mathrm{Ti}$ molar ratios of up to 10 . The continuous hydrothermal reactor used in this report, ${ }^{602}$ involved feeding of the Ti-sol and $\mathrm{LiOH}$ solutions in separate feeds to be premixed in flow before being fed into a reactor at flow rate of $5 \mathrm{~mL} \cdot \mathrm{min}^{-1}$. This mixed feed was then introduced to a feed of superheated water $\left(40 \mathrm{~mL} \cdot \mathrm{min}^{-1}\right)$ to give reaction temperatures in the range $350-410{ }^{\circ} \mathrm{C}$. The combined flows then passed through a $13.5 \mathrm{~m}$ long $1 / 8^{\prime \prime}$ heated reactor zone (set at temperatures in the range 350 to $410^{\circ} \mathrm{C}$ ) in order to provide a sufficient residence time for the reaction to occur. The NPs which formed were then cooled in flow and recovered. ${ }^{602}$ BET surface area data revealed specific surface areas in the range of 98 to $116 \mathrm{~m}^{2} \mathrm{~g}^{-1}$ for phase pure LTO. After heat-treatment at 400 and $600^{\circ} \mathrm{C}$, the BET surface areas were ca. 83 and $38 \mathrm{~nm}$, respectively. Although this report showed some nice results for LTO synthesis control, no electrochemical property data was reported.

Other titanate anodes for Li-ion batteries can also be made by manufacturing the corresponding sodium-ion electrode material and then replacing the sodium with lithium via an electrochemical or other methods (e.g. in the cell). This can often yield structures for Li-ion materials that are inaccessible under normal synthesis conditions. For example, $\mathrm{Na}_{2} \mathrm{Ti}_{3} \mathrm{O}_{7}$ is an interesting sheet like anode material for Li-ion batteries because the analogous $\mathrm{Li}$-ion material $\mathrm{Li}_{2} \mathrm{Ti}_{3} \mathrm{O}_{7}$ (orthorhombic space group $\mathrm{Pbnm}$, Ramsdellite-type) does not form the same crystal structure. It may be expected that lithiation of the sodium form would potentially give access to the $2 \mathrm{D}$ structure for intercalation. The authors previously reported the synthesis of photoactive $\mathrm{Na}_{2} \mathrm{Ti}_{3} \mathrm{O}_{7}$ (see section 7.1). 475 An interesting feature of making this in CHFS is that the material is does not form a rolled up parchment structure as it does under batch hydrothermal processing. This rolling up has been linked to a need to reduce strain in the sheets as they are formed and evidently, the speed of synthesis and recovering the products made in CHFS, are largely in an unrolled form with the edges exposed. In the most recent report by the authors, a material with the nominal formula " $\mathrm{Na}_{2} \mathrm{Ti}_{3} \mathrm{O}_{7}$ " was made via CHFS. As it was found to be sodium deficient and assigned the formula $\mathrm{H}_{1.1} \mathrm{Na}_{0.9} \mathrm{Ti}_{3} \mathrm{O}_{7}$ in which both $\mathrm{Na}^{+}$and $\mathrm{H}^{+}$ ions reside between the anionic layers, provide overall charge balance. The flow synthesis was carried out using 0.3 
$\mathrm{M}$ TiBALD, $12 \mathrm{M} \mathrm{NaOH}$ and a supercritical water flow at $c a .450^{\circ} \mathrm{C}$. Samples were used as the electrode active materials without any further post-synthesis heat-treatment of any kind. Cyclic voltammetry of the material, which was evaluated in a half-cell in the potential range of 0.05 to $3.0 \mathrm{~V} \mathrm{vs} \mathrm{Li/Li+}$ suggested that the material showed significant pseudocapacitance behavior. For cycling tests, at a current rate of $50 \mathrm{~mA} \cdot \mathrm{g}^{-1}$, the specific capacity was ca. 220 mAh. $\mathrm{g}^{-1}$, but showed very poor coulombic efficiency (59\%), which was be attributed to electrolyte destruction at very low potentials beyond $0.5 \mathrm{~V}$. At higher applied currents of $500 \mathrm{~mA} \cdot \mathrm{g}^{-1}$, the cycle life remained excellent and the columbic efficiency was always $>99.6 \%$ for the first 1200 cycles measured (at which point the specific capacity was > $\left.110 \mathrm{mAh} \cdot \mathrm{g}^{-1}\right)$. At current rates of up to $10 \mathrm{Ag}^{-1}$ it was even possible to obtain a specific capacity of as much as 38 mAh.g-1 (Figure 43)

Figure 43

The above-mentioned titanates containing $\mathrm{Li}$ or $\mathrm{Na}$ ions, often require relatively forcing conditions to manufacture them in continuous hydrothermal flow processes, however, it is still possible to manufacture materials such as titanias and doped titanias (or other host anode lattices) in high yield, which can also function as high power anodes for Li-ion batteries. Pure anatase $\mathrm{TiO}_{2}$ anode made using continuous hydrothermal synthesis have been evaluated as anodes. The half cells were cycled galvanostatically at applied currents ranging from $0.1 \mathrm{~A} \cdot \mathrm{g}^{-1}(\mathrm{ca} .0 .5 \mathrm{C}$ charge or discharge rate which is in ca. 30 minutes) to $10 \mathrm{~A} . \mathrm{g}^{-1}$ which was $c a .60 \mathrm{C}$ (i.e. 1 minute charge or discharge rate), in the potential range 1 to $3.0 \mathrm{~V}$ vs $\mathrm{Li} / \mathrm{Li}^{+}{ }^{283}$ This $\mathrm{CHFS}$-made nano- $\mathrm{TiO}_{2}$ showed excellent rate performance with a capacity of $88 \mathrm{mAh} . \mathrm{g}^{-1}$ at $5 \mathrm{~A} \cdot \mathrm{g}^{-1}$ and $70 \mathrm{mAh} . \mathrm{g}^{-1}$ at $10 \mathrm{~A} \cdot \mathrm{g}^{-1}$, which is comparable to or better than other powdered anatase titania anodes for Li-ion batteries in the literature.

More recently, the authors used a CHFS reactor for the fabrication of high Power Nb-doped $\mathrm{TiO}_{2}$ (25 at\% dopant) NP anodes for lithium-ion batteries. ${ }^{583}$ Again it is worth mentioning that the high level of dopant achieved in the titania lattice, is far in excess of that which would normally be expected, which allows very unique (metastable) compositions and properties to be obtained. The as-prepared nanopowders with $\mathrm{Nb}$ dopant $(<6 \mathrm{~nm})$ were used to make printed anodes without any further heat-treatment or processing at all after the synthesis process other than freeze drying. The nanomaterials were made into electrodes in half cells versus lithium chip and were initially investigated via cyclic voltammetry and galvanostatic charge/discharge cycling tests in the range 1.2 to $3.0 \mathrm{~V}$ vs $\mathrm{Li}^{\mathrm{L}} \mathrm{Li}^{+}$. 
Interestingly, the $\mathrm{Nb}$-doped $\mathrm{TiO}_{2}$ samples showed superior capacity retention at high current rates compared to the corresponding undoped nano- $\mathrm{TiO}_{2}$. For example, cycling the cells at current rates of 5 and ${ }_{15} \mathrm{~A} \cdot \mathrm{g}^{-1}$, the doped material (bracketed undoped $\mathrm{TiO}_{2}$ values) showed a specific capacity of $105 \mathrm{mAh} \cdot \mathrm{g}^{-1}$ (88 mAh.g $\mathrm{g}^{-1}$ undoped) and $48 \mathrm{mAh} . \mathrm{g}$ ${ }^{1}$ (27 mAh.g $\mathrm{g}^{-1}$ undoped), respectively. ${ }^{583}$

The superior performance at high currents for the doped titania sample was believed to be due to a combination of the higher electronic conductivity and a greater charge storage contribution from pseudocapacitive charge storage (i.e. Faradaic surface chemical processes and possibly some Helmholtz double layer charge storage). ${ }^{83}$ Interestingly, the results from the same report, suggested that the BET surface area was not the most critical parameter for high power performances. Therefore, the combined effect of higher electronic conductivity, improved lithiumion kinetics and higher capacity for charge storage via surface effects, benefitted the high power performance compared to the undoped material..$^{58}$

A further benefit of using a simple host material such as nano-titania as an anode material for Li-ion batteries, is that the lattice can be doped over a wide range of concentrations to improve electrochemical performance in a number of ways; the dopant can improve the conductivity of the titania, which can improve the rate performance. Secondly, the dopant can stay in the lattice and it could alter the mechanism of charge storage under certain rate conditions, such as increasing the pseudocapacative contributions due to forming stronger interactions between the dopant and with Li-ions near the surface (see before for Nb doped titania). ${ }^{83}$ Thirdly, the dopant can act to widen the electrochemical potential range of the anode material, generally via becoming a separate phase which undergoes its own reactions after the first cycle (as will be seen from Sn doped titania below ${ }^{283}$ ).

The authors recently reported the laboratory scale continuous hydrothermal production of titania and Sn doped titania derivatives, which are potential anodes for Li-ion batteries. ${ }^{283}$ The metal salt precursor solution containing acidified titanium oxysulphate hydrate and tin sulphate (aq.) in the appropriate ratio (pump flow rate $=40 \mathrm{~mL}^{\mathrm{min}} \mathrm{m}^{-1}$ ) was premixed with a $\mathrm{KOH}$ (aq.) feed $\left(40 \mathrm{~mL} \cdot \mathrm{min}^{-1}\right)$ at room temperature in a dead volume tee piece. This combined feed was then brought into contact with a flow of supercritical water at $c a .400^{\circ} \mathrm{C}$ and $24.1 \mathrm{MPa}\left(80 \mathrm{~mL}\right.$. $\left.\mathrm{min}^{-1}\right)$ to form the products in a confined jet reactor, CJM under a highly turbulent mixing regime (Reynolds number was ca. 6500, residence time $c a .5 \mathrm{sec}$ ). The base concentration was kept constant throughout at $0.0703 \mathrm{M}$ and the total metal salt concentration of $0.0625 \mathrm{M}$, and the titanium salt was used with a concentration of $0.0625,0.06,0.575$ and $0.055 \mathrm{M}$ (with the Sn precursor making up any shortfall). The actual amounts of Sn measured in the samples were 
close to this in the precursor feeds and were given as follows (with the Sn precursor nominal levels given in brackets; $\mathrm{TiO}_{2}$ (from o at\% $\mathrm{Sn}$ in precursor), $\mathrm{Ti}_{0.94} \mathrm{Sn}_{0.06} \mathrm{O}_{2,}$ (from 4 at\% $\mathrm{Sn}$ in precursor), $\mathrm{Ti}_{0.89} \mathrm{Sn}_{0.11} \mathrm{O}_{2,}$ (8 at\% $\mathrm{Sn}$ in precursor) and $\mathrm{Ti}_{0.85} \mathrm{Sn}_{0.15} \mathrm{O}_{2}$ (12 at\% $\mathrm{Sn}$ in precursor). ${ }^{29}$ After synthesis, the samples were freeze-dried and then used directly for anode inks as-prepared with no further processing. This later point is in stark contrast to many other anode and cathode materials in this review, which often require a further post synthesis high temperature heat-treatment step in order to realize high electrochemical performance.

XRD data for all Sn doped titania samples suggested the presence of phase pure anatase $\mathrm{TiO}_{2}$ type structure [similar to similar to JCPDS reference pattern 21-12729] with no additional peaks in the patterns (e.g. such as those for $\mathrm{SnO}_{2}$ or other impurities), suggesting that a metastable phase had been stabilized. ${ }^{29}$ Doping appeared to reduce the peak intensity significantly, suggesting highly strained or defective doped crystallites (a small shift towards lower angles was observed which was due to the dopant). The BET surface area decreased with higher Sn doping content $\left(\mathrm{TiO}_{2} 245 \mathrm{~m}^{2} \mathrm{~g}^{-1}, \mathrm{Ti}_{0.94} \mathrm{Sn}_{0.06} \mathrm{O}_{2} 230 \mathrm{~m}^{2} \mathrm{~g}^{-1}, \mathrm{Ti}_{0.89} \mathrm{Sn}_{0.11} \mathrm{O}_{2} 208 \mathrm{~m}^{2} \mathrm{~g}^{-1}, \mathrm{Ti}_{0.85} \mathrm{Sn}_{0.15} \mathrm{O}_{2} 178 \mathrm{~m}^{2} \mathrm{~g}^{-1}\right)$ corresponding to the increase of the average crystallite size. ${ }^{283}$ The influence of Sn doping in nano-sized $\mathrm{TiO}_{2}$ on the rate performance and specific capacity at different current rates at two different potential ranges was explored (these were 1.0 to $3.0 \mathrm{~V}$ vs $\mathrm{Li}_{/} \mathrm{Li}^{+}$and 0.05 to $3.0 \mathrm{~V}$ vs $\mathrm{Li} / \mathrm{Li}^{+}$, respectively). In the absence of in situ $\mathrm{X}$-ray measurements in the cell to determine the fate of the $\mathrm{Sn}$ in the doped samples, the authors interpreted the cyclic voltammetry as pointing to the likely processes going on in the Sn doped titanias when cycled in the wider voltage window towards $0.05 \mathrm{~V} \mathrm{vs} \mathrm{Li}_{/} \mathrm{Li}^{+}$.

The authors suggested that during the first reduction cycle, the $\mathrm{Sn}^{4+}$ in the anatase structure was reduced at low potentials to form a separate phase of elemental tin $\left(\mathrm{Sn}^{\circ}\right)$ with $\mathrm{Li}_{2} \mathrm{O}$ being irreversibly formed (this was indeed accompanied by a high irreversible initial capacity loss). It was then suggested that the elemental tin behaved thereafter like a separate alloying material, i.e. the nanomaterial effectively became a $\mathrm{Sn}$ and $\mathrm{TiO}_{2}$ nanocomposite anode in which the $\mathrm{TiO}_{2}$ appeared to effectively buffer the high volume expansion of the dispersed $\mathrm{Sn}$ formed in situ. Thus, the Sn phase could be lithiated in the range 1.0 to $0.05 \mathrm{~V} \mathrm{vs} \mathrm{Li/Li+}$ for form $\mathrm{Li}_{x} \mathrm{Sn}$ alloy (where $0<x \leq 4.4$ ).

Delithiation of the samples in the potential range 0.05 to $3.0 \mathrm{~V} \mathrm{vs} \mathrm{Li/Li+} \mathrm{(forward} \mathrm{sweep} \mathrm{in} \mathrm{the} \mathrm{CV)} \mathrm{showed} \mathrm{a} \mathrm{broad}$ oxidation peak at $0.5 \mathrm{~V}$ vs $\mathrm{Li}_{/} \mathrm{Li}^{+}$which was suggested due to the delithiation of the $\mathrm{Li}_{x} \mathrm{Sn}$ (de-alloying reaction). Thus, the $\mathrm{Sn}$ was largely active below $1.0 \mathrm{~V}$ vs $\mathrm{Li} / \mathrm{Li}^{+}$with the $\mathrm{TiO}_{2}$ phase being active in the range ca. 1.0 to $3.0 \mathrm{~V}$ vs $\mathrm{Li} / \mathrm{Li}^{+} .29$ 
The sample with anode active phase composition $\mathrm{Ti}_{0.85} \mathrm{Sn}_{0.15} \mathrm{O}_{2}$ had a capacity of ca. $350 \mathrm{mAh} . \mathrm{g}^{-1}$ at applied constant currents of $100 \mathrm{~mA} \cdot \mathrm{g}^{-1}$ and a capacity of $192.3 \mathrm{mAh} \cdot \mathrm{g}^{-1}$ at a current rate of $1500 \mathrm{~mA} \cdot \mathrm{g}^{-1}$ (Figure 44 ). After 500 charge / discharge cycles (at a higher constant current rate of $382 \mathrm{~mA} \cdot \mathrm{g}^{-1}$ ), the same nanomaterial anode retained a relatively high specific capacity of $240 \mathrm{mAh} . \mathrm{g}^{-1} .^{29}$ Given the long-term retention of a decent specific capacity for some of these compositions, the Sn doped titanias offer an interesting balance of moderate power and capacity anodes.

Figure 44

Other Metal Oxide/Sulfide Anodes (Nb, V, Sn, Co, Nb, Fe, Sb, Mo, etc.). Nano-sized, semi-crystalline niobium pentoxide $\left(\mathrm{Nb}_{2} \mathrm{O}_{5}\right)$ was synthesized in a single step via a continuous hydrothermal process with o.1 $\mathrm{M}$ ammonium niobate $(V)$ oxalate hydrate used as only precursor. ${ }^{603}$ The as-prepared material displayed $98.6 \%$ capacity retention after 800 cycles) and showed promising high rate performance, with a specific capacity of $43 \mathrm{mAh} . \mathrm{g}^{-1}$ at an applied current of $10 \mathrm{~A} . \mathrm{g}^{-1}$ (in the wide potential range of 0.05 to $3.0 \mathrm{~V} \mathrm{vs} \mathrm{Li/} / \mathrm{Li}^{+}$). Scan rate tests were used to investigate the proportion of stored charge from diffusion-limited processes and that from surface effects (pseudocapacitance); these experiments clearly showed that at higher currents, charge storage from the latter was dominant. ${ }^{603}$

In a further report by the UCL authors, the performance of undoped $\mathrm{VO}_{2}$ nano-sheets made via continuous hydrothermal route was improved further. ${ }^{604}$ The $\mathrm{VO}_{2}$ nano-sheets showed excellent capacity retention, with a specific capacity of $350 \mathrm{mAh} . \mathrm{g}^{-1}$ at an applied current of $0.1 \mathrm{~A} \cdot \mathrm{g}^{-1}$ and $95 \mathrm{mAh} \cdot \mathrm{g}^{-1}$ at $10 \mathrm{~A} \cdot \mathrm{g}^{-1}$ in the potential range 0.05 to 3.0 V vs. Li/Li+. Additional electrochemical testing suggested that a significant proportion of the charge storage in the cells was due to pseudocapacitive processes. ${ }^{604}$ Therefore, many of the synthesized insertion/intercalation anode materials synthesized via continuous hydrothermal routes showed far improved high power performances just due to the facilitated particle morphology, crystallinity and size.

Based on the aforementioned promising results for $\mathrm{Nb}_{2} \mathrm{O}_{5}$ and also $\mathrm{Sn}$ doped titania, the authors used continuous hydrothermal processes to make $\mathrm{Sn}$ doped niobium oxide in the compound $\mathrm{Nb}_{1.63} \mathrm{Sn}_{0.34} \mathrm{O}_{5}$ (production rate ca. 130 g.h-1 ${ }^{-1}{ }^{605}$ Pure $\mathrm{Nb}_{2} \mathrm{O}_{5}$ made under similar conditions had a specific capacity of $191 \mathrm{mAh} . \mathrm{g}^{-1}$, which was lower in comparison to $\mathrm{Nb}_{1.66} \mathrm{Sn}_{0.34} \mathrm{O}_{2}\left(272 \mathrm{mAh} \cdot \mathrm{g}^{-1}\right.$ at $\left.100 \mathrm{~mA} \cdot \mathrm{g}^{-1}\right)$. Again, the high capacity had to be due to the combination of 
the charge storage reactions for the $\mathrm{Nb}$ oxide as well as the $\mathrm{Sn}$ oxide. The specific capacities of $\mathrm{Nb}_{1.66} \mathrm{Sn}_{0.34} \mathrm{O}_{5}$ at different current rates were $181 \mathrm{mAh} . \mathrm{g}^{-1}$ at $500 \mathrm{~mA} \cdot \mathrm{g}^{-1}, 146 \mathrm{mAh} \cdot \mathrm{g}^{-1}$ at $1.0 \mathrm{~A} \cdot \mathrm{g}^{-1}$ and $110 \mathrm{mAh} . \mathrm{g}^{-1}$ at $2.0 \mathrm{~A} \cdot \mathrm{g}^{-1} .605$ The Coulombic efficiency was $<95 \%$ at $100 \mathrm{~mA} \cdot \mathrm{g}^{-1},>98.5 \%$ at $500 \mathrm{~mA} \cdot \mathrm{g}^{-1}$ and $>99.4 \%$ at higher currents. The capacity remained quite unstable during a further 100 cycles at $500 \mathrm{~mA} \cdot \mathrm{g}^{-1}$ with capacity retention of $71 \% .605$

Similar to the work on $\mathrm{Nb}$ oxide, recent work from some of the authors has shown that vanadium oxide doped with $\mathrm{Sn}$, namely $\mathrm{V}_{0.8} \mathrm{Sn}_{0.2} \mathrm{O}_{2 \text {, }}$ (production rate of $65 \mathrm{~g} \cdot \mathrm{h}^{-1}$ ) could display increased charge storage due to the electrochemical activation of the Sn-dopant. The compound had a large specific capacity ${ }^{605}$ of 674 mAh.g $\mathrm{g}^{-1}$ (at $100 \mathrm{~mA} \cdot \mathrm{g}^{-1}$ ) and in the variable C-rate test ( 5 cycles per C-rate) the sample had capacities of 673 mAh.g $g^{-1}$ at 100 A.g $g^{-1}, 607$ mAh.g ${ }^{-1}$ at $500 \mathrm{~mA} \cdot \mathrm{g}^{-1}, 560 \mathrm{mAh} \cdot \mathrm{g}^{-1}$ at $1,000 \mathrm{~mA} \cdot \mathrm{g}^{-1}$ and $515 \mathrm{mAh} \cdot \mathrm{g}^{-1}$ at 2,000 mA.g. $\mathrm{g}^{-1}$. These capacities were far higher compared to the pure $\mathrm{VO}_{2}\left(255 \mathrm{mAh} . \mathrm{g}^{-1}\right.$ at $\left.100 \mathrm{~A} \cdot \mathrm{g}^{-1}\right){ }^{605}$ The coulombic efficiency was $<97 \%$ (after 4 cycles) at $100 \mathrm{~mA} \cdot \mathrm{g}^{-1}$ and $>99.5 \%$ at higher currents. The higher reversible capacities were due to possible particle cracking during cycling (enabling more accessible Li-ion storage sites) and higher delithiation activities at higher potentials. Thus, the authors could show that for three different classes of insertion / intercalation anode nano-materials (namely $\mathrm{TiO}_{2}, \mathrm{Nb}_{2} \mathrm{O}_{5}$ and $\mathrm{VO}_{2}$ ), the capacity can be increased via doping of $\mathrm{Sn}$ in the crystal lattice within the same synthesis method / step.

There have also been reports of nano-crystalline $\mathrm{SnO}_{2}$ containing materials, which might be of interest in a Li-ion or Na-ion battery. The material was made in the range 385 to $415^{\circ} \mathrm{C}$ ( $30 \mathrm{MPa}$ ) using a residence time range of 38 to $106 \mathrm{~s}$ in a tubular flow reactor. ${ }^{606} 0.1$ to $0.4 \mathrm{M} \mathrm{SnCl}_{4}$ was used as a reagent with a particle size of ca. $3 \mathrm{~nm}$ and yields in the range 53 to $81 \%$, (or $98 \%$ when $0.1 \mathrm{M} \mathrm{NaOH}$ was added). After heat-treatment at $450^{\circ} \mathrm{C}$ for $2 \mathrm{~h}$, the particles were only ca. $4 \mathrm{~nm}$, whilst a heat-treatment at $600{ }^{\circ} \mathrm{C}$ for $10 \mathrm{~h}$, improved crystallinity with a particle size of ca. 9 $\mathrm{nm} .{ }^{606}$ In another report, $\mathrm{SnO}_{2}$ shell on a titania core ${ }^{158}$ was were developed via a new dual stage reactor by Iversen and co-workers. Due to the very small size of the $\mathrm{SnO}_{2}$ obtained, such materials may be ideal as anodes for $\mathrm{Li}$ or $\mathrm{Na}$ ion batteries. If the nano- $\mathrm{SnO}_{2}$ can be reduced ex-synthesis, then the $\mathrm{Sn}$ on the titania, may prove to be a useful material in this regard.

Another interesting anode material $\mathrm{CoSb}_{2} \mathrm{O}_{4}$ that has been made rapidly under hydrothermal conditions (in a batch system that essentially mimics flow) was reported in an in situ high pressure PXRD study by Iversen and colleagues, which combines the idea of conversion and alloying electrode materials. The material was scaled up via a more conventional reaction and then evaluated as an anode in a Li-ion half-cell showing an initial discharge capacity 
of $1131 \mathrm{mAh} . \mathrm{g}^{-1}$, corresponding to $\sim 15 \mathrm{Li}$ atoms reacting with each $\mathrm{CoSb}_{2} \mathrm{O}_{4}$ (some of the Li-ions will alloy with the

Sb and some will form a lithium oxide / hydroxide type species). ${ }^{607}$ The first charge capacity of the sample was 417 mAh. $\mathrm{g}^{-1}$, going down to $\sim 20 \mathrm{mAh} . \mathrm{g}^{-1}$ after the tenth cycle. Thus this material still requires some development if it is to compete with the alternative anodes which have been reported earlier. This was an impressive publication because the authors were able to use in situ synchrotron PXRD to follow the formation and growth of $\operatorname{CoSb}_{2} \mathrm{O}_{4} \mathrm{NPs}_{\mathrm{s}}$ using different precursor stoichiometries. ${ }^{607}$

Continuous hydrothermal processes have also been used to make materials which could have applications as anodes (but were not tested for such yet), e.g. $\operatorname{MoS}_{2} \cdot{ }^{122}$ In the first reported continuous hydrothermal synthesis of $\mathrm{MoS}_{2}$, the reaction of ammonium heptamolybdate with preheated thiourea (with in flow addition of acid) required an additional heating unit in process between the mixer (reactor) outlet and the cooling system. This gave an additional residence time of $30 \mathrm{~s}$ at a set temperature of $250^{\circ} \mathrm{C}$ in order to produce relatively phase pure $\mathrm{MoS}_{2}$ directly in process (rather than amorphous $\mathrm{MoS}_{3}$, which is initially produced at lower temperatures). Larger size $\mathrm{MoS}_{2}$ is of interest as an inorganic lubricant due to its extensive 2D or sheet like structures, which allow exfoliation and can consequently significantly reduce friction. The sheet-like structures are also idea for intercalation of Li ions. Overall, the huge number of possible anode materials based on (alkali-) (transition) metal oxides (sulfides) shows already endless opportunities when using a continuous hydrothermal synthesis route.

\subsection{Beyond Li-ion Batteries}

Due to the expense and potential future fears on the scarcity of lithium, a number of global industries and researchers are also conducting research into alternatives such as sodium, magnesium, metal-air, metal-sulfur, and metal-organic batteries. Some advantages of Na-ion batteries are as follows;

(i) Na-ion materials have lower material costs than Li-ion materials

(ii) Cathode and electrolyte costs can be reduced (these can be as much as ca. $50 \%$ of cell costs),

(iii) $\mathrm{Na}$ is far more abundant in the earth's crust than $\mathrm{Li}(\mathrm{Na} \sim 2.6 \% \mathrm{vs}$. $\mathrm{Li} \sim 0.005 \%)$.

(iv) Na-ion materials can be processed just like Li-ion materials, from the synthesis of the active materials to the electrodes that will allow utilizing of existing cell fabrication lines and

(v) More inexpensive current collectors such as Al based ones can be used in sodium-ion cells. 
The advantage of cost of raw material and sustainability ( $\mathrm{Na}$ vs Li salt) has important implications for continuous hydrothermal synthesis because many battery materials are indeed made with an excess of $\mathrm{Li}$ or $\mathrm{Na}$ in the process and if not all of it is incorporated into the particles, it must then be recovered from the waste water streams.

Li-ions have the smallest ionic radius ( $0.76 \AA \AA$ ). During charge and discharge, Li-ions can readily intercalate into a wide range of hosts. Considering that solid state diffusion is the major rate-determining step of electrochemical reactions, the small ionic size permits fast kinetics Furthermore, the small mass number for Li (6.941 amu) and low redox potential (-3.04 V vs. standard hydrogen electrode, SHE), enable high theoretical specific capacity and energy density of rechargeable energy storage systems. Na-ions (1.02 Å) are only slightly larger that Li-ions in Group 1, therefore it has broadly similar intercalation / alloying / conversion chemistry as well as a higher redox potential $\left(-2.71 \mathrm{~V}\right.$ vs. SHE) of $\mathrm{Na} / \mathrm{Na}^{+}$. Thus, $\mathrm{Li}$ and $\mathrm{Na}$-ions have different interactions host structures, which can influence the kinetics as well as thermodynamics.

To the knowledge of the authors, there no published examples on the use of CHFS made Na-ion batteries. The authors are actively working in this area and will report their findings in due course. It is expected that high power capabilities will be achievable for $\mathrm{Na}$-ion nanostructured anodes that would show a shift from battery-like (intercalation) behavior at low C-rates to oxide supercapacitor-like behavior at higher C-rates. Thus, Na-ion (and Al or Mgion) batteries, offer the potential to develop metal-ion chemistries in the future which do not rely on Li-ions, which may become expensive or in short supply. The authors are convinced that making suitable $\mathrm{Na}$-ion or multivalent-ion nano-sized electrodes via methods such as continuous hydrothermal flow will be a critical breakthrough technology to allow such materials to actually work and compete or exceed Li-ion materials in terms of power and energy density.

\subsection{New Supercapacitor Nanomaterials}

Next generation electrochemical capacitors (ECs) are now emerging as a promising energy storage technology for high power applications. ${ }^{608}$ The weakness of EC systems is the limited energy density, which restricts applications to delivery of high power over timescales of a few seconds. Thus, much of the current research has investigated methods to improve both the power and the energy capacity of such devices. An excellent review by Dunn et al. ${ }^{609}$ is recommended as it highlights the many opportunities for investigating nano-sized supercapacitor materials, most of which can be readily made via CHFS. 
Since the energy density is a product of capacitance and the voltage (squared), many strategies are focused in how to increase these values. So called traditional electrochemical double-layer capacitors (EDLCs) store charge in the Helmholtz double layer (electrical double layer) which is situated at the interface between the electrode and electrolyte. Such devices are known for their very long cycle lives and high stability. Most commonly, various types of activated carbons, which have very high surface areas or large number of defects of dangling bonds or with porosity to allow ionic diffusion, are used in EDLCs in order to increase the size of the interface, which increases capacitance. However, more recently, other materials are being increasingly used such as electroactive polymers and NP metal oxides, the latter of which have the potential for very high capacitances in excess of 1000 Farads per gram $\left(F . g^{-1}\right)$, which is far higher than the values that carbons can produce (see figure 45 )

Figure 45

In particular, it appears that carbon incorporated nano-sized metal oxides such as $\mathrm{RuO}_{2}, \mathrm{MnO}_{2}, \operatorname{Ir}-\mathrm{Mn}$ oxides, $\mathrm{NiO}$, Co oxides are attracting a great deal of interest in this regard. Already, several groups around the world have reported the continuous hydrothermal synthesis of similar materials but not tested them in such applications to date. $^{19,119,136,142,166,446,534-535,610-614}$

The literature contains examples of various amorphous or nanomaterials, which are suitable as supercapacitor electrodes; in the case of hydrous $\mathrm{RuO}_{2}$ prepared by sol-gel methods, the high capacitance was attributed to the hydrated surface layers that promoted facile electron and proton transport (such high capacitance was not stable during high rate cycling). Such materials (with differing levels of hydration) could probably be made via continuous hydrothermal methods with careful control of reaction conditions such as temperature. Furthermore, CLOC oxidation catalysts made by CHFS and based on Ru-Ti oxides 535 might well be worth investigating as supercaps as well due to the fine dispersion of Ru that is obtained and accessible.

A recent trend in supercapacitor materials is the use of nano-sized metal oxides that are integrated with carbons, which seems promising for high stability as well as high power. This could present an ideal opportunity for researchers working in continuous hydrothermal synthesis because such processes have the potential to generate carbon (normally from fructose or sucrose) in situ, which may facilitate the manufacture of carbon-inorganic core/shell or nanocomposites such as has been reported to Li-ion cathode materials recently. ${ }^{586}$ 
Recently, there has been a move towards asymmetric hybrid devices that combine an electric double layer capacitor electrode (mainly double layer charge storage) combined with a battery type anode (pseudocapacitive), which can give a better balance of high power and high energy density. ${ }^{608}$ Based on promising results mentioned earlier on high power anode materials made from continuous hydrothermal, $283,583,603$ which showed that charge storage mechanisms of these anodes were largely pseudocapacitive, the authors have begun to explore CHFS made NPs as high surface area electrodes for supercapacitors. Hybrid supercapacitors can be made from nano-sized anatase and doped titanias and used directly in hybrid Li-lon asymmetric supercapacitors. In principle, doped $\mathrm{TiO}_{2}$ could be used as the negative electrode material and activated carbon (AC) as positive electrode. It would be expected that such hybrid supercapacitors will possess good energy densities at moderate power densities (see Ragone plot in figure 38 for the expected performance curves for CHFS nanomaterials hybrid supercapacitors versus other types of energy storage devices). However, at the time of writing this review, not a single report on hybrid supercapacitors using any CHFS NPs, has been published (although research is underway in UCL).

It may be even able to get better performance when metal oxide nanoparticles are electronically in contact with well-structured porous graphene's. Kaner and co-workers have investigated the design of high-energy-high-power hybrid supercapacitor electrodes. A number of typical designs are shown in Figure 46A-D. They suggested that improving the ionic current (IC) and electronic current $(E C)$ within the electrode is a key. They have compared different approaches including compact thick films (Figure 46A), nanostructured metal oxide films (Figure 46B), addition of conductive materials with nano metal oxide (Figure 46C) and growing or depositing a nanostructured metal oxide on 3D interconnected graphene network with high surface area and high electronic conductivity (Figure 4D). They suggest that only the latter design offers the best potential for high-energy and high-power hybrid supercapacitor electrode performance (in their case for hybrid microcapacitors). Therefore, structuring the active and any conductive carbons or networks within an electrode, will be important. CHFS methods will be able to offer the possibility of integrated carbons and actives in this regard in the future no doubt.

Figure 46

In closing, there are many opportunities in the development of new nanostructures and high surface area CHFS materials and carbons for supercapacitors. Significant gains may be possible in terms of "getting more charge stor- 
age with less material", i.e. by nano-sizing precious metal oxides such as $\mathrm{RuO}_{2}$, or by also doping materials, which are less expensive, it may be possible to increase capacity to cost ratio significantly.

\section{MISCELLANEOUS NANOMATERIALS CHEMISTRY AND PROCESS DEVELOPMENTS}

For purposes of completeness, this section will cover some of the miscellaneous applications and uses and development of continuous hydrothermal that were not discussed in any detail in earlier sections.

10.1 Misc Precursors, Co-solvents, Additives for Novel Chemistry or Phase Targeting

In the past several groups have discussed the effect of additives in the reagent feeds in order to control redox properties or to control particle nucleation and growth kinetics inside the continuous hydrothermal. Such innovations are set to continue as researchers push the boundaries of what might be possible inside continuous hydrothermal reactors. Great care should be taken to understand the effects of reagents and additives; in particular, the use of stronger reducing agents in situ, may enable an expansion of the current capabilities of what metallic materials can be made in situ. Use of alcohols and molecules such as ammonia are also set to allow the change in critical temperatures and perhaps even the composition of the final oxide or oxynitride materials as they are obtained from the systems themselves.

Obtaining a particular NP product from continuous hydrothermal is not simply a case of heating metal salt and base / modifier solutions to supercritical conditions; indeed, a number of synthetic strategies have been developed to allow greater control of materials properties. As mentioned earlier, a third HPLC pump can be used in process in order to add a flow of base solution or other additives prior to nucleation, during nucleation, or during growth. For example, iron nitrate hydrolyses to hematite at $573 \mathrm{~K}$, but adding urea (which decomposes to $\mathrm{NH}_{3}$ under hydrothermal conditions) via a third pump, produces ferrihydrites. ${ }^{134}$ The quantity of base added can also affect the product; for example, in the production of yttrium aluminum garnet doped with Tb (YAG:Tb), the concentration of base $(\mathrm{KOH})$ is critical to achieving a phase pure product. ${ }^{14^{1}}$ The $\mathrm{R}$ ratio (ratio of the concentration of hydroxide ions to the total concentration of all other anions), has to be 1.0. At $\mathrm{R}=0.7, \mathrm{AlO}(\mathrm{OH})$ is produced as an impurity phase, whereas at $\mathrm{R}=1.2, \mathrm{YO}(\mathrm{OH})$ appears as an impurity. The results by Hakuta et al. demonstrate that varying the quantity of base in a continuous hydrothermal reaction, can be used as a strategy to access different phases. For example, in a continuous hydrothermal system with superheated water at $623 \mathrm{~K}$ (flow rate $\left.20 \mathrm{~mL} \cdot \mathrm{min}^{-1}\right)$, a $0.1 \mathrm{M} \mathrm{Co}\left(\mathrm{NO}_{3}\right)_{2}$ solution 
mixed with $0.2 \mathrm{M} \mathrm{KOH}$ (both precursor feeds at $10 \mathrm{~mL} \cdot \mathrm{min}^{-1}$ ) hydrolyses to form $\mathrm{Co}(\mathrm{OH})_{2}$, whereas under the same conditions but with a base concentration of $1.0 \mathrm{M}, \mathrm{CoO}(\mathrm{OH})$ is formed. Likewise, with either 0.1 or $1.0 \mathrm{M}$ base (but at $723 \mathrm{~K}$ for the superheated water inlet temperature), $\mathrm{Co}_{3} \mathrm{O}_{4}$ and $\mathrm{CoO}$, respectively, are formed. ${ }^{136}$

The type of the counter ion (anion) is also important for continuous hydrothermal chemistry, as quite often under such severe conditions, anions can be broken down to form reducing or oxidizing gases in situ. These molecules can affect the particular oxide or other phases that are obtained. For example, when iron(III) sulphate or nitrate salts are hydrolyzed at $673 \mathrm{~K}$, hematite $\left(\mathrm{Fe}_{2} \mathrm{O}_{3}\right)$ is formed, whereas ammonium iron(III) citrate decomposes to form a partially reduced oxide form, magnetite $\left(\mathrm{Fe}_{3} \mathrm{O}_{4}\right) \cdot{ }^{72}$ This is because the breakdown products of the citrate ion $\left(\mathrm{C}_{6} \mathrm{H}_{5} \mathrm{O}_{7}{ }^{3-}\right)$ are reducing in nature. Likewise, acetate salts tend to favor reduction. Nitrate ions can form oxidizing gases such as $\mathrm{NO}_{x}$ and $\mathrm{OH}$ radicals; ${ }_{i}^{615}$ when $\mathrm{Co}\left(\mathrm{NO}_{3}\right)_{2}$ reacts with superheated water in a continuous hydrothermal system, $\mathrm{Co}_{3} \mathrm{O}_{4}$ tends to be the major product, ${ }^{72}$ whereas if $\mathrm{Co}(\mathrm{Ac})_{2}$ is used, $\mathrm{CoO}$ results (where all the $\mathrm{Co}$ is in $2+$ oxidation state). ${ }^{135}$

There have been several examples of additives being used to create a reducing or oxidizing atmosphere within the mixing point of the continuous hydrothermal system. For example, $\mathrm{H}_{2} \mathrm{O}_{2}$ breaks down under hydrothermal conditions to form a number of oxidizing species such as $\mathrm{O}_{2}$ and hydroxyl radicals $\left(\mathrm{OH}^{\bullet}\right)$. The first use of $\mathrm{H}_{2} \mathrm{O}_{2}$ in continuous hydrothermal reactions was in the synthesis of $\mathrm{LiCoO}_{2}$, where $\mathrm{H}_{2} \mathrm{O}_{2}$ was added to the superheated water feed in order to fully oxidize $\mathrm{Co}^{2+}$ to $\mathrm{Co}^{3+} .{ }^{668} \mathrm{H}_{2} \mathrm{O}_{2}$ does not solely act as an oxidant; in the hydrolysis of $\mathrm{Co}\left(\mathrm{NO}_{3}\right)_{2}$, using a superheated water feed of $623 \mathrm{~K}$ with $1 \mathrm{M} \mathrm{KOH}$ (without $\mathrm{H}_{2} \mathrm{O}_{2}$ ), $\mathrm{CoO}(\mathrm{OH})$ was formed. In contrast, when $\mathrm{H}_{2} \mathrm{O}_{2}$ was added to the metal salt feed, $\mathrm{CO}_{3} \mathrm{O}_{4}$ was formed as the exclusive product. ${ }^{136}$ In the case of $\mathrm{CoO}(\mathrm{OH})$, $\mathrm{Co}$ is in a $3^{+}$oxidation state, whereas for $\mathrm{CO}_{3} \mathrm{O}_{4}$, the average oxidation state is $+2^{2} / 3$. This indicates that peroxide, or its breakdown products, are not solely acting as oxidants, but may be facilitating dehydration under such conditions.

In contrast to oxidizing molecules, a mildly reducing atmosphere can also be created in situ by adding other reagents, such as formic acid $\left(\mathrm{H}_{2} \mathrm{CO}_{2}\right)$, which breaks down into $\mathrm{CO}_{2}$ and $\mathrm{H}_{2}$. Formic acid was used in a continuous hydrothermal reaction by Sue et al. to produce metallic Ni directly from Ni(Ac) ${ }_{2}{ }^{86}$ Interestingly, the Ni metal NPs did not appear to precipitate homogeneously from solution; instead, the NPs were precipitated onto a $\mathrm{Fe}_{3} \mathrm{O}_{4}$ core nanomaterial that was produced in the flow process at an earlier mixing point.

As well as using water alone, researchers have also made use of other solvents such as alcohols (or water/alcohol mixtures) as the process liquid phase. ${ }^{203}$ Alcohols are often used because they can aid the solubility of the precursors 
initially and / or because they may have a beneficial crystallising effect on the products, 94 due to their lower critical temperatures compared to water (e.g., for $\mathrm{scMeOH}_{1} \mathrm{~T}_{\mathrm{c}}=240^{\circ} \mathrm{C} / \mathrm{P}_{\mathrm{c}}=81 \mathrm{bar}^{28}$ ).

10.2 Surface Functionalised Hybrid NPs and Nanocomposites

In natural composites, we are familiar with the bonded interactions between hard (inorganic) and soft (organic or polymer) biomaterials being mixed on the nano or atomic levels. In nature this give rise to natural composites (e.g. bone, teeth, etc.) with novel and impressive combinations of mechanical and biological properties. In some materials such as calcium phosphates, binding of surface agents (such as phosphonates for example), can occur under relatively mild conditions. ${ }^{616}$ Surface functionalisation can also be achieved in flow reactors under relatively mild conditions in minutes $\left(<100^{\circ} \mathrm{C}\right), 35^{30}$ however, for the vast majority of papers herein, we will discuss surface functionalisation of large oxide NPs under supercritical flow conditions. metal-organic frameworks (MOFs) $516,617-620$ which are usually a 3D network of inorganic and organic ensembles to form a true atomic level hybrid, will be dealt with in a separate section in this review (part 10.4). The area of carbons and their treatment in continuous hydrothermal processes, is largely beyond this review as there is a large diverse literature on biomass or other chemicals in such processes (batch supercritical processes for developing high quality graphenes from coal are known though ${ }^{621}$ )

Surface-Functionalized Nanoparticles. Surface-functionalized nanoparticles, SFNs (also described as hybridorganic-inorganic nanomaterials by Adschiri and others. ${ }^{25,36,54,65-66,137,217,290,298,429,444,524,622-624}$ These will be called SFNs hereafter and can be thought of as inorganic NPs (e.g. a metal or metal oxide) which have a surface coating of some organic species, preferably one with appropriate ligands that are added in the process in the ideal case. The process of surface functionalization of agents into the surface of a NP can be done in either batch of flow processes or indeed or indeed it can be done post process (via refluxing or chemical coupling reactions). ${ }^{312}$ An excellent review by Aymonier and colleagues on "creation of interfaces in composite/hybrid nanostructured materials using Supercritical Fluids" is recommended for an overview of this area in terms of how surface engineering can be used to develop inorganic-inorganic and inorganic-organic nanostructured interfaces for advanced composites. ${ }^{622}$

Adschiri and colleagues have undertaken many short batch reactions in the presence of organic capping agents in order to given suggestions about what may be possible in flow systems with extended residence times (several minutes). For example, organic-functionalized nanoscale yttrium aluminium garnet (YAG) nanoparticles were syn- 
thesized in ca. 10 minutes at $420^{\circ} \mathrm{C}$ (followed by rapid quenching). ${ }^{193}$ Reactions were carried out in the presence of oleylamine and oleic acid as organic surface modifiers (6:1 molar ratio of organic agents to total Yb $+A l)$. The presence of the organic molecules resulted in YAG particles with various morphologies including cubic, spherical and chainlike. Because of their functional groups, the organic reagents allowed the particles to disperse in certain organics solvents. In related batch experiments, the synthesis of surface functionalised $\mathrm{ZrO}_{2}$ was achieved from the reaction of $\mathrm{Zr}(\mathrm{OH})_{4}$ with various surface modifiers (oleic, sebacic, dodecanedioic, and 12 aminododecanoic acids) at 400 ${ }^{\circ} \mathrm{C}$ for $10 \mathrm{~min}$. As expected, a reduction in particle size versus non-modified reactions, was observed (due to inhibition of growth due to surface binding in the former). ${ }^{290}$

More relevant to this review, surface functionalization has also been carried out in continuous hydrothermal processes (i.e. during the nucleation or growth of particles in flow). There are a number of proposed routes to in process direct production of SFNs which have been developed and articulated primarily by Professor Adschiri's pioneering research and more recently by others and these generally fall under the following categories;

Type 1; pumping a slurry of the preformed material to be coated into a stream superheated water feed and adding a surface agent at some point,

Type 2; making NPs from the reaction of a metal salt, which is also in the presence of a surfactant or adding this surfactant in a second mixer that is downstream of the first reaction (Figure 47 shows a schematic flowchart for making SFN particles from solution and then add a capping agent in a second mixer)

Type 3; gradually increasing the temperature of a NP/surfactant feed in a long residence time reactor set-up.

Reduction agents can also be added the sc-water feed before it reacts with the metal salt to get a metallic SFN. Using a type 2 SFN approach, Teja et al. prepared surface coated iron oxide $\left(\alpha-\mathrm{Fe}_{2} \mathrm{O}_{3}\right)$ from precursor solutions of PVA and iron(III) nitrate, which were fed to meet a flow of sc-water and then into a crystallizer zone. The presence of PVA during particle formation was an effective way to obtain narrow particle size distribution as it limited particle growth and coalescence under certain condtions. ${ }^{137}$ The results showed that the average particle size decreased 
with increasing PVA concentration when the residence time was ca. $2 \mathrm{~s}$. This became nearly independent of PVA concentration when the residence time was much longer at $>10$ S. ${ }^{137}$

Figure 47

Using a type 2 approach for SFN synthesis, Adschiri et al. reported attempts to surface coated cerium oxide with either L-glutamic acid, L-aspartic acid, or L-arginine. ${ }^{625}$ This was conducted by pumping an aqueous solution of the metal nitrate in the presence of the surface modifiers at a relatively modest temperature of $275^{\circ} \mathrm{C}$ in a flow reactor (presumably, at higher temperatures there may have been significant degradation of the surface agents). ${ }^{625}$ The products synthesized with L-glutamic acid of primary $\mathrm{CeO}_{2} \mathrm{NPs}$ bound together by the surface agent to give larger cubic-like nano-assemblies. ${ }^{625}$ In a related report, Adschiri et al. the formation of hierarchical cubic nanoassemblies of octahedral $\mathrm{CeO}_{2}$ nanocrystals was described; in the report, an aqueous solution of cerium(III) nitrate with hexanedioic acid was heated in a lab-scale plug-flow reactor. ${ }^{624}$ The main aim behind such ideas is to create intelligent hierarchical systems for nano-devices, however, there may ultimately be other interesting uses for such assemblies such as in the reduction or control of sintering of catalyst upon ageing for example (e.g. in TWCs 493,495$)$ There are of course variants of this approach. However, the authors suggest that the consensus is that the temperature for capping should not be too high and ideal conditions for capping will depend on residence time and mixer turbulence for an effective surfactant-coating window in the approximate range 180 to $250^{\circ} \mathrm{C}$.

Figure 48 shows a batch process example where the expected phase behavior for water and organic solvents containing a surface agent and water containing metal salts at a) room temperature, b) supercritical conditions when the two phases can become homogenous and c) back to room temperature. The metal oxide nanoparticles are formed under supercritical conditions via hydrolysis and dehydration in the presence of the surfactant for form surface functionalized nanoparticles which can be separated in the organic phase (if sufficiently hydrophobic). ${ }^{117}$ In Figure $48 \mathrm{~d}$, a dispersion of oleic acid capped cobalt blue NPs from the Adschiri laboratory that are dispersed in hexane. ${ }^{298}$ Thus, if we assume that under continuous (rather than batch) hydrothermal conditions, it would also be expected that any organic phase (or hydrophobic surfactant) and the superheated aqueous phase may become more homogenous; this would also favor the formation of SFNs in flow (although this may take a few minutes in 
some cases). As in the above batch example shown in Figure 48 , if hydrophobic surface agents are added during the synthesis of NPs in flow, then, at the end of the process (after cooling), the SFNs would be expected to separate from the aqueous layer.

Figure 48

As an alternative to in-process capping, post-synthesis surface modification can also be conducted, e.g. silanecoupling agents are known to stick organic groups onto the surface of particles. This relies on the reaction of pendant silane groups with surface OH groups on the particles, giving Si-O groups on the NP surface, which could affect properties. Thus, there has been interest in synthesizing SFNs with organic agents that are able to use either charge or steric stabilization so particles can be formulated into inks or dispersed into polymers. $36,117,290,350,623,626$

By far the most developments in SFNs for flow (and batch) syntheses, has been carried out by Adschiri and coworkers. They have shown tremendous innovation and versatility in this area and pushed towards commercial applications. ${ }^{68,299,625,627-628}$ One of the most interesting observations made by Adschiri et al., is that highly coated SFNs behave very differently to uncoated (or partially coated) particles. ${ }^{299}$ It appears that by coating the entire surface of the NP with an organic "sheath" effectively makes the particle present a more oleophillic exterior to the surrounding solvent or polymer it is dispersed in. In a ceramic ink such as that used for inkjet printing for example, Prof Adschiri has suggested that this can result is inks that do not appreciably shear thicken as expected over a wide range of shear rates. ${ }^{299}$ Examples of SFNs in the literature include alumina oxyhydroxide, $\mathrm{TiO}_{2}{ }^{54}$ copper manganates, ${ }^{524}$ boron nitrides, ceria, $\mathrm{Co}$ oxide spinels, nickel oxide, $\mathrm{CoAl}_{2} \mathrm{O}_{4}{ }^{298}$ pigments amongst others; in all these cases, the surface functionalization also reduce the particle size and reduced agglomeration significantly. ${ }^{299}$

In 2010, Adschiri and co-workers reported the synthesis of surface functionalized (oleic acid) Ba-hexaferrite nanocrystals with controlled size and morphology. 456 In the process, a solution of iron nitrate and barium hydroxide (Ba/Fe molar ratio of 0.5 ) was mixed with $\mathrm{KOH}$ in distilled water to give an initial hydroxide that was made up to 0.05 M. $45^{6}$ In a separate feed the oleic acid was made in the range 0.25 to $0.5 \mathrm{M}$ in ethanol. The metal salt first met with sc-water flow to give a reaction temperature in the range 450 to $500^{\circ} \mathrm{C}(30 \mathrm{MPa})$ and this mixture was heated in flow (residence time 20s) before meeting the oleic acid feed to give a reaction temperature in the range 350 to $380^{\circ} \mathrm{C} .456$ This was then cooled to give the capped products. It was shown that the oleic acid reagent played a key role in con- 
trolling shape of Ba-hexaferrite as well as giving a drastic reduction in particle size from 30 to $9 \mathrm{~nm}$. The surface functionalized nanocrystals exhibit a high coercivity of ca. 2800 Oe at 5 and $280 \mathrm{~K} .456$

In Japan, a company called ITEC Co. Ltd has been set up which offers capabilities for lab and larger scale facilities called Momicho-mini, Mega and Giga from smallest to largest) examples given on the company website. ${ }^{627,}{ }^{629}$ The industrial SFNs (and functionalized microparticles) are made via continuous hydrothermal processes include surface functionalized $\mathrm{CeO}_{2}, \mathrm{LiFePO}_{4}, \mathrm{LiMn}_{2} \mathrm{O}_{4 l} \mathrm{BaTiO}_{3}$, AlN, barium ferrite, various metal nanoparticles. Furthermore, it was suggested that organic modification can also be made on ready-made particles of aluminum oxide $\left(\mathrm{Al}_{2} \mathrm{O}_{3}\right)$ and boron nitride (BN). ${ }^{118,} 299,627-628$ Other than that, a few reports that have emerged on SFNs, much of the research may indeed be commercially sensitive (and therefore may remain unpublished to date) as this group has been working with numerous companies to develop a wide range of NPs for composites.

In 2010, Hayashi and co-workers reported the synthesis of cubic hybrid barium titanate $\left(\mathrm{BaTiO}_{3}: \mathrm{BT}\right) \mathrm{NPs}$ coated with polyacrylic acid (PAA) or Tween 80 [polyoxyethylene(20) sorbitan mono-oleate]. Such coated particles might be ideal for inkjet printable inks for dielectric device applications or supercapacitors. ${ }^{429}$ For the synthesis of hybrid $\mathrm{BaTiO}_{3}$, a TiO 2 sol and $\mathrm{Ba}\left(\mathrm{NO}_{3}\right)_{2}$ aqueous mixture (2.5:3.0 molar ratio, i.e. excess $\mathrm{Ba}$ ) was mixed in flow with $\mathrm{KOH}$ (aq), before the combined flow met with a supercritical water feed in a tee mixer. This mixture was then heated in flow in a furnace and then introduced to the ambient temperature aqueous modifier solution (containing the surface agent) before in process cooling. As in many other reports, due to the excess of Ba used, the as-made material was washed with diluted acetic acid solution and pure water to remove surface $\mathrm{BaCO}_{3} .429$ Thermogravimetric analysis (in the temperature range 200 to $600^{\circ} \mathrm{C}$ ) of the samples coated with PAA and Tween 80 , exhibited weight losses due to the ligands of ca. $11.2 \%$ and $19.5 \%$, respectively, (exothermic peak centered at ca. $350^{\circ} \mathrm{C}$ ), indicating that surface modification on the particles was significant.

More recently, the UCL authors and others have begun to develop the science and different methods of adding surface agents in flow, i.e. in the same process. ${ }^{36,129,324,623,630-631}$ Generally, it appears that the addition of a surfactant using a second mixer (upstream of NP formation), is a noteworthy approach as it gives a lot of process flexibility for scoping out changes in dispersion with a range of factors..$^{6}$ The UCL authors have also found that when some surface coverage is achieved for ITO and doped ZnO particles in ink bases, it is possible to keep modest viscosities and reduce caking of the inks (long term stability) which makes them better for inkjet printing. Work is currently underway to assess their conductivities after processing via low temperature routes. In Figure 49 non-citrate coated 
(transparent) conducting oxide nanoparticles can be seen, which can be made into inks that don't form homogenous mixtures that are suitable for inkjet printing. On the lower part of Figure 49 , high loading ceramics inks can be made for inkjet printing in excess of $40 \mathrm{wt} \%$ or more when surface functionalisation is imparted on the particles.

Figure 49

Surface coatings of inorganic coatings on inorganic cores (i.e. core-shell particles) have also begun to emerge and it has been noted that such inorganic coatings can confer improved dispersion or other properties on the core materials.632 For example, gamma- $\mathrm{Fe}_{2} \mathrm{O}_{3} @ \mathrm{SiO}_{2}, \mathrm{TiO}_{2} @ \mathrm{SiO}_{2}$, and alpha- $\mathrm{Fe}_{2} \mathrm{O}_{3} @ \mathrm{SiO}_{2}$ core-shell NPs were made by Iversen et al. using a continuous flow dual-stage reactor. In the products, uniform silica shells of $2 \mathrm{~nm}$ thickness were obtained (on gamma- $-\mathrm{Fe}_{2} \mathrm{O}_{3}$ and $\mathrm{TiO}_{2} \mathrm{NPs}$ ) while more irregular shells were deposited on alpha- $\mathrm{Fe}_{2} \mathrm{O}_{3}$ due to the low $\mathrm{pH}$. The silica coating was added using TEOS (in isopropanol) which was added after nucleation of the core and cooling. After the TEOS was added, the mixture was then heated in a secondary reactor. ${ }^{632}$

SFNs for Nanocomposites. Nanocomposites are hybrid organic inorganic materials, in which mixing of the NP filler phase is achieved, ideally at the sub $100 \mathrm{~nm}$ level. Nanocomposites have generated much research interest owing to remarkable enhancements in the properties at very low volume fractions (typically $<10$ vol\%. This morphology of nanoscale dispersion can lead to tremendous interfacial contact between the NPs with the polymer matrix. This effectively confines the polymer chains, giving synergistic improvements in the composite properties (increased modulus, strength, etc.), where the overall properties are superior to those of the individual components.

The properties of nanocomposites are directly affected by values such as the filler volume fraction, particle aspect ratio, surface coating, agglomeration of particles, alignment in the composite and other considerations. If such composites can in fact be bonded to the polymer after mixing, then of course the resulting composites will being to resemble natural nanocomposites to some degree (although this would also require some form of hierarchical structure of the composite as well to be truly comparable).

It appears that some of the high loading composites are being driven by the need to make improved composites. Conventionally in composites, when the filler content is increased above $50 \mathrm{wt} \%$, the viscosity can increase which makes it difficult to process into a final form. Adschiri has suggested that surface modification of BN particles by a 
continuous hydrothermal approach improves the affinity for polymers, resulting in $>90 \mathrm{wt} \% \mathrm{BN}$ content composite being processable (without any void formation) that possesses a thermal conductivity of $40 \mathrm{Wm}^{-1} \mathrm{~K}^{-1}$ (order of magnitude higher than the currently used materials). Despite such high loadings, the devices that were made from this SFN BN were flexible and insulating with good ease of fabrication. $\mathrm{SFN} \mathrm{Al}_{2} \mathrm{O}_{3}$ particles have also been developed for packaging materials and transparent dispersion of high refractive index nanocomposites (with $\mathrm{SFN} \mathrm{TiO}_{2}$ and $\mathrm{ZrO}_{2}$ ) have been developed, in polymers with good transparency using new monomer, (with a pendant chelating $\mathrm{OH}$ groups) which affords a high loading ratio up to $70 \mathrm{wt} \%$ of $\mathrm{ZrO}_{2}$ in a composite. Despite some information being available, it is likely therefore that due to commercial sensitivities, some of the research will not reach the chemical literature fully. ${ }^{299}$

One of the most recent areas where we are aware of the using of continuous hydrothermal process for particle coating of NPs, has been in the field of composite fire retardants. Egyptian researchers investigated the use of surface coated fire retardants, which were made using continuous hydrothermal processes. ${ }^{630-631,633} \mathrm{~A}$ number of materials were investigated for similar applications including surface functionalized titanias, hydroxyapatites and AlOOH particles which were readily dispersible in epoxy resins giving better dispersions that uncoated particles. ${ }^{633}$

Magnesium di-hydroxide (MDH) was mixed in polymer composites and shown to have a significant impact on flammability performance. MDH rods of ca. $120 \times 20 \mathrm{~nm}$ were made and incorporated into composites and from the loss of water upon exposure to heat from the metal hydroxide, MDH was used as a endothermic heat sink. Hydroxyapatite (surface modified) was also investigated as a fire retardant in a recent report by the same researchers..$^{630}$ CHFS-made aluminum-oxide-hydroxide $(\mathrm{AlOOH})$, was also recently published as a clean and non-toxic flame retardant. The phase transition of $\mathrm{AlOOH}$ to $\mathrm{Al}_{2} \mathrm{O}_{3}$ was demonstrated by X-ray and enhanced flammability properties were achieved in polymer composites at low solids loading. ${ }^{633}$ Finally, for the calcium phosphates with an intumescent agent known as Exolit AP750 in a multi-component epoxy nanocomposite, was able to resist a flame at $1700^{\circ} \mathrm{C}$ and self-extinguish after the flame had been removed, which was significantly better than virgin epoxy.

Aymonier and co-workers developed a continuous process for the manufacture of Talc, a clay mineral $\left[\mathrm{Mg}_{3} \mathrm{Si}_{4} \mathrm{O}_{10}(\mathrm{OH})_{2}\right] .{ }^{634}$ Talc is not only used in automotive plastics and household appliance plastics, as well as in window profiles, food packaging, polypropylene pipes, laptop housings. ${ }^{635}$ In these applications, talc can increase rigidity, creep strength and impact resistance. In the report for talc synthesis, aqueous feeds of sodium metasilicate and a feed of magnesium acetate (in acetic acid) were mixed in flow in a tee mixer that caused an initial precipitation. This 
mixture then entered a heated coil zone (volume of $8 \mathrm{~cm}^{3}$ ) at a temperature up to up to $500{ }^{\circ} \mathrm{C}$ that was followed by cooling in flow before being collected in a filter downstream. ${ }^{634}$

\subsection{Nanograined Coatings and Monoliths}

Surface functionalized or unagglomerated NPs can be used as a feed material for nano-grained coatings or for the fabrication of nanograined ceramic monoliths. ${ }^{127,128,451}$ Surface functionalized nanoparticles can readily dispersed in an appropriate liquid medium that can act as a delivery vehicle (via an aerosol or a spray) for transport towards heated substrates or some kind of flame or plasma torch that facilitates thermal and kinetic energy to the particles. ${ }^{37}$

Thermal Spraying. Thermal spraying is one of a number of techniques including chemical vapor deposition and physical vapor deposition, which have been used to produce NP coatings (Figure 50)37. These processes utilize high temperature heating / flame / plasmas to deposit particles on substrates. A reducing atmosphere in such processes is often caused by incomplete combustion of fuel gases (in flame processes ${ }^{41-43}$ ), or via the use of hydrogen (in plasmas). One of the drawbacks of plasma dry powder coating processes is that the feed of powders require careful size selection to only use large micro-sized particles otherwise, compaction can occur in the feed hopper and the feed becomes blocked. This means that pre-sieving of powders is needed that adds cost and makes powder particle size rather inflexible. This has led to interest in methods that allow any size of particles to be introduced from microns to NPs, such as suspension spray techniques. ${ }^{37,}{ }^{6} 6$ In such techniques, the powder has to be first dispersed into a liquid phase such as water for example. This suspension is then sprayed onto a surface via a flame or plasma to remove the liquid by evaporation and provide the energy to make particles agglomerate, neck, sinter or melt and then stick onto a substrate such as stainless steel. 37

The continuous hydrothermal process, preferably with the presence of surface coated additives is an ideal feedstock for thermal suspension spraying, because as mentioned above, it is possible to manufacture coated particles which can be stable in dispersions. ${ }^{37}$ The UCL authors recently reported the use of Suspension Plasma Spraying (SUPS) for the deposition of conformal titanium dioxide coatings. The process utilizes a CHFS-made NP slurry of $\mathrm{TiO}_{2}$ (ca. 6 and $12 \mathrm{~nm}$ in size, respectively) in water, which was fed into a high temperature plasma jet (ca. 7000 to 20 $000 \mathrm{~K}$ ) in a spray system, ${ }^{37}$ which allowed deposition of nanostructured titanium dioxide coatings. The coatings were 
produced by varying the feedstock crystallite size, spray distance and plasma conditions. The coatings produced exhibited anatase phase contents in the range ca. 90 to $100 \%$, with the remainder being rutile. The coatings had a granular surface consisting of densely packed agglomerates interspersed with some melted material. All of the coatings were shown to be photoactive by means of a sacrificial hydrogen evolution test under UV radiation and compared favorably with reported values for CVD coatings and compressed discs of commercial photocatalyst $\mathrm{P} 25 \cdot{ }^{37}$

Figure 50.

NP Consolidation / Sintering Methods. In the manufacture of nanograined ceramics, methods of sintering NPs without grain growth, are important. Perhaps the single most useful method here is spark plasma sintering that can be used to densify nanoparticles made via continuous hydrothermal methods. ${ }^{127,} 128,357,426,439,451$ Importantly, the rapid heating rates and short heating times, allow for fast densification without significant or extensive grain growth to occur, which offers the promise of stronger or novel nanograined all-ceramic monoliths or nanocomposites.

Alternative to conventional heat-treatments for sintering, include the use of microwave heat treatments in order to improve the inter-particle connections..$^{382}$ Such methods can afford high heating rates and often lower temperatures for sintering. This is still a relatively underdeveloped area, which holds much promise for the future, particularly for printing onto thermally sensitive substrates.

10.4. Hybrid Metal-Organic Frameworks (MOFs)

Much of this section will focus on nano-sized Metal-Organic Frameworks (MOFs) made via continuous hydrothermal processes, which have the capacity to store gases or indeed chemicals, some of which have energy, environmental or indeed healthcare applications. Key applications for MOFs are in $\mathrm{CO}_{2}$ capture / storage, $\mathrm{H}_{2}$ storage, catalysis and pharmaceutical drug delivery. MOFs consist of an inorganic unit and an organic linker, which are bonded to create a 3D lattice, which usually has very large pore volumes and low densities. Normally, MOFs are synthesized using batch methods (hydrothermal and solvothermal) and many of the reports in flow systems are not purely or wholly water based, however, they are mentioned here as they offer a new development in flow reactor solvothermal processes. Some notable reports for semi-continuous synthesis of MOFs have recently been reported by Schoenecker and co-workers for the synthesis of UiO-66 (using a batch process which allowed removal of products 
and introduction of reagents). ${ }^{516}$ Subsequently, MOFs, of HKUST-1 and CPO-27(Ni) were reported using the Continuous hydrothermal synthesis method (using the counter-current mixer) of Lester and co-workers. ${ }^{516}$

Figure 51

The group of Lester, have made significant efforts in the synthesis of MOFs and found that under a wide range of conditions up to $400{ }^{\circ} \mathrm{C}$, (from ambient pressure to $240 \mathrm{bar}$ ) the choice of reagents and the resulting $\mathrm{pH}$ levels are critical in obtaining the desired product to yield particles on the micro- and nanoscales. MOFs in many cases require long residence times for syntheses and temperatures do not always need to be so high, which is good for process intensification purposes. Notably, for the copper(II) carboxylate compound, HKUST-1 (commercial name Basolites C300), the material could be made highly crystalline with a residence time of only $1 \mathrm{~s}$ or so (see Figure $51 \mathrm{c}$ for the profile fit to a powder X-ray pattern of HKUST-1 sample prepared at $300{ }^{\circ} \mathrm{C}$ with $0.03 \mathrm{M} \mathrm{Cu}^{2+}$ ). The analogous batch process for HKUST-1 is typically performed at $<150^{\circ} \mathrm{C}$. In the flow process, materials made at $300{ }^{\circ} \mathrm{C}$, showed some variation in size and shape of crystals as the concentration and flow rate of the reagents was changed, with larger crystals seen at lower dilution. In the same report for the synthesis of $\mathrm{CPO}-27(\mathrm{Ni})$, nitrogen sorption analysis showed a type I isotherm with a BET surface area of ca. $1030 \mathrm{~m}^{2} \cdot \mathrm{g}^{-1}$. Furthermore, the $\mathrm{CPO}-27(\mathrm{Ni}) \mathrm{MOF}$ was shown to uptake $\mathrm{CO}_{2}$ gas at $19.57 \mathrm{wt} \%$ in tests, which is similar to data for samples from conventional syntheses (despite the fact that the material was made in a fraction of the time). ${ }^{516}$ Figure 51 a shows a TEM image of CPO-27(Ni) prepared at 300 ${ }^{\circ} \mathrm{C}$ and high concentration, whilst Figure $51 \mathrm{~b}$ shows a SEM image of the same material made at $200{ }^{\circ} \mathrm{C}$, prepared at lower concentration)

Other notable reports for the continuous synthesis of MOFs has included the following; Rubio-Martinez and coworkers, who reported the continuous synthesis of HKUST-1, UiO-66 and NOTT-400 using a flow reactor with Tpiece mixing point (followed by a long residence time heating stage under 7 bar pressure). ${ }^{618}$ For a typical reaction in the flow process, solutions of $0.1 \mathrm{M} \mathrm{Cu}\left(\mathrm{NO}_{3}\right)_{2} \cdot 3 \mathrm{H}_{2} \mathrm{O}$ and $0.24 \mathrm{M}$ benzene-1,3,5-tricarboxylic acid (BTC) in ethanol, were mixed under flow conditions and heated in a tubular reactor. The synthesis was conducted at $140^{\circ} \mathrm{C}$ using a total flow rate of $90 \mathrm{~mL} \cdot \mathrm{min}^{-1}$, which gave a residence time of $1.2 \mathrm{~min}$ (yield $100 \%$ ). A similar tee-piece reactor with post-heating was used to continuously make a MOF based on cerium-terephthalate ${ }^{619}$ (100 Bar pressure) and MIL$53(\mathrm{Al})$ made in the temperature range $250-300{ }^{\circ} \mathrm{C}$ and a pressure of 230 bar. ${ }^{617}$ In the work on cerium- 
terephthalate ca. $500 \mathrm{~nm}$ highly crystalline mesoparticles based in $\mathrm{Ce}_{5}\left(\mathrm{OOC}-\mathrm{C}_{6} \mathrm{H}_{4}-\mathrm{COO}\right)_{7.5}(\mathrm{DMF})_{4}$ were made in $30 \mathrm{~s}$ rather than the $12 \mathrm{~h}$ required for the analogous batch solvothermal process at $150^{\circ} \mathrm{C}$ (batch process gives large $\sim 10$ $\mu \mathrm{m}$ particles. This is evidently an area, which is set to continue to develop, however, it will be important to conduct reactions, which use water rather than organic solvents if MOF type materials are to be commercialized and able to compete with batch processes.

10.5 In Situ Flow / Rapid Heating Studies

In the literature, in situ X-ray studies have been widely used in both solid-state chemistry and wet-chemistry to study a variety of reactions and processes, but until recently, studies under supercritical conditions were few and mainly limited to static experiments. A number of groups have now begun to develop special experiments or analytical techniques in which they can follow the nucleation, growth and evolution of NPs under flowing, rather than more conventional batch hydrothermal conditions. In some cases, rapid batch system heating is applied, which simulates what can occur in a flow situation, whilst in other situations, flow reactors are employed and the data can be collected at various locations to gain spatial and temporal information.

The use of in situ X-ray methods with customized reactors (batch injection type with rapid heating using hot air or a heated block design are typical) and in some cases flow processes) to follow nucleation and crystal development, has been particularly impressively developed by Iversen and Aymonier and co-workers. In 2014, Iversen and colleagues reviewed the use of powder diffraction and total scattering methods for in situ studies of solvothermal and hydrothermal reactions in, which experimental setups used for in situ X-ray and neutron studies, were presented along with and methods of data analysis. In specific reports, Iversen et al. used specially built apparatus setups to carry out X-ray scattering (and related in situ) experiments to reveal information on process kinetics for batch and flow experiments. $161,173,175,177-180,289,326,365,425,442,447,485,607,637-638$ In 2008 , this team also reported a reactor design for in situ X-ray scattering studies of nanoparticle formation in supercritical water. $447,63^{8}$ of relevance to this review, in the report, synchrotron X-rays were used to investigate a reaction occurring inside either a sapphire or a stainless steel 316 tubing (partially surrounded by a heating block with access for allowing X-rays in and out). The apparatus used as well as typical data generated, is shown in figure 52 
Figure 52

A number of different designs and requirements for in situ XRD have been proposed including designs in which the metal salt solution is not pre-heated, but instead is rapidly heated in a block in flow in seconds. 447 For example, the group demonstrated the growth of $\mathrm{Fe}_{3} \mathrm{O}_{4}$ nanoparticles (synthesized using $0.50 \mathrm{M}$ ammonium iron(III) citrate at 350 ${ }^{\circ} \mathrm{C}$ and $300 \mathrm{bar}$ ) that were monitored using a set up for both Small Angle X-ray Scattering (SAXS) and Wide Angle Xray Scattering (WAXS) data on the same detector. By using peak broadening data, they were able to estimate crystallite size along the tube and effectively reveal that after ca. $10 \mathrm{~s}$, a sudden doubling in particle size was observed. This apparatus clearly shows the benefit of being able to perform in situ analysis experiments in understanding nucleation and growth processes. The same authors have also suggested that other complimentary methods (to WAXS and SAXS) such as refined or modelled PDF (Pair Distribution Function) and XAS (X-ray Absorption Spectroscopy) may also be of use to provide information about the local structure interatomic distances of crystalline or indeed amorphous nanoparticles formed during nucleation/growth. 447

Studies comparing both batch and flow in situ experiments have been reported by Aymonier and colleagues, including the production of $\mathrm{BaTi}_{1-y} \mathrm{Zr}_{y} \mathrm{O}_{3}(0 \leq \mathrm{y} \leq 1, \mathrm{BTZ})^{425}$ nanocrystals that is a challenging system due the low reactivity of zirconium reagents. As previously, the team used in situ synchrotron wide angle X-ray scattering (WAXS) analyses in batch (at $150^{\circ} \mathrm{C}$ ) and flow (at $400^{\circ} \mathrm{C}$ ) to follow, the development of BTZ nanomaterials in real time; the work highlighted the complexity of the nucleation and growth mechanisms in this system $)^{425}$

In other designs in which in situ X-ray diffraction was conducted, a flow reactor was employed in which preheated water feed was mixed with an ambient temperature metal salt feed in the conventional way. ${ }^{442}$ The researchers found that coupling of in situ synchrotron PXRD with ex situ spectroscopy and other analyses, e.g. FTIR (Fourier Transform Infrared Spectroscopy), Raman Spectroscopy, XPS (X-ray photoelectron spectroscopy), ex-situ X-ray diffraction and high resolution transmission electron microscopy, led to a better understanding of particle growth behaviors, in particular, highlighting temperature and composition effects. ${ }^{42^{2}}$ Other notable work involving in situ PXRD methods by Iversen and colleagues include the (rapidly heated in a batch sapphire tube) hydrothermal synthesis of $\mathrm{CoSb}_{2} \mathrm{O}_{4}$, which is of interest as a conversion Li-ion anode material. ${ }^{607}$

Other materials that have been investigated for rapid heating (to simulate conditions similar to what may be seen in flow); these include an in situ total X-ray scattering study of the formation of $\mathrm{WO}_{3}$ nanoparticles, ${ }^{177}$ the syn- 
thesis of $\mathrm{Pt}$ and $\mathrm{Pt}_{3} \mathrm{Gd}$ nanoparticles under solvothermal conditions, ${ }^{180}$ and the evolution of $\mathrm{TiO}_{2}$ nanoparticles (from an alkoxide precursor) under hydrothermal conditions. Prior to these reports, in 2014, the same team of researchers also investigated in situ total scattering studies of zinc oxide ${ }^{176}$ and iron oxide (maghemite, $\gamma-\mathrm{Fe}_{2} \mathrm{O}_{3}$ from ammonium iron(III) citrate) also. ${ }^{175}$ Other notable reports by the same team include the first comprehensive structural description of the solvothermal formation of yttria-stabilized zirconia (YSZ), from its precursor in solution to the final crystal (using an array of in situ methods). ${ }^{174}$

Yoko et al. investigated the use of situ high-energy X-ray diffraction measurements to look at reactions occurring in millisecond timescales for barium zirconate $\left(\mathrm{BaZrO}_{3}\right)$ at $400{ }^{\circ} \mathrm{C}, 30 \mathrm{MPa}$. First-principles calculations were used to support the proposed formation mechanism for $\mathrm{BaZrO}_{3}$, which involved nuclei consisting of mainly zirconium oxide to absorb the barium ions during crystallization. ${ }^{639}$

Tomographic and imaging methods under extreme flow reactor conditions are also of interest to better understand flows and how they mix. Some of the UCL authors in collaboration with the UCL Industrial Materials group at $U C L$, undertook a collaboration to try and take a closer look inside a counter-current reactor mixing zone (where supercritical water and the metal salts meet). ${ }^{640-641}$ The authors built a specially commissioned portable continuous hydrothermal reactor system that was small enough to be housed on a large $X-Y$ stage at the high-energy beamline ID15A of the European Synchrotron Radiation Facility in Grenoble, France. ${ }^{641}$ Tomographic Energy Dispersive Diffraction Imaging (TEDDI) was applied through the $1 / 4$ inch stainless steel counter current mixing zone in which tomographic images were obtained while the material was actually nucleating in flow over a $12 \mathrm{~h}$ period and images were recorded (assuming steady state) for ceria NPs made from cerium ammonium nitrate, CAN, solution. In effect, each data point was like a unique XRD pattern; by application of the Scherer equation, a crystallite size map was built-up.

An imaging capability: "T/EDDI" was also used in which a combination of tomographic angle and energy dispersive diffraction imaging, was realized and used to uncover the nature of crystallization growth processes occurring within the counter-current mixing zone. The technique revealed the buildup of $\mathrm{CeO}_{2}$ during synthesis and, importantly, it was able to precisely locate the main crystallization zone for a counter-current mixer. In contrast to these "point by point" methods, which create a time averaged "image" of what is going on, researchers have used neutron imaging (of a Tee piece mixer) to gain insights into the behavior of mixing under supercritical conditions due to the different densities of the hot and ambient temperature feeds ${ }^{161-162}$ (see Figure 5a). Such methods can clearly show any effects of buoyancy or other forces and also be used as a good tool for finding ideal conditions. 
The use of high throughput (HT) synthesis and screening techniques to speed up the discovery process is well established, with pharmaceutical companies being the first to invest heavily into the concept. ${ }^{64^{2}}$ The key is to test as many samples as possible for indicative properties rather than conduct comprehensive characterisation on each sample. This type of approach can be either driven by or explained by use of appropriate computational methods to develop structure-property-compositional relationships. . $^{63-646}$

The authors were inspired to develop High Throughput Continuous Hydrothermal (HiTCH) flow reactors to be able to meet the need to rapidly make libraries of nanoceramic samples. Much of the inspiration to develop HiTCH flow reactors came from a research project led By Professor Julian Evans (then at Queen Mary University London) developed an automated discovery robot called LUSI (London University Search instrument) in which ceramic inks were mixed and homogenized prior to printing and firing (to bring about a solid state reaction) to make combinatorial libraries. ${ }^{647}$

The LUSI concept which worked well for some ceramic materials such as mixed titanates, however, in selected cases, incomplete mass transfer occurred in mixtures upon firing so that certain phase pure heterometallic materials or solid solutions were not accessible (as further heat and grind steps were needed to homogenize the compositions). This can in fact be a perennial problem in solid-state materials science where two precursor oxides are mixed and fired to obtain a product. Several other groups have also tried to conduct combinatorial ceramic materials research using liquid or homogenous sources of metal ions in order to make compositional grids. ${ }^{643-646}$ However, such processes are invariably limited due to lack of homogenous metal distribution unless very careful crosslinking or gelation approaches are used in the pre-firing stage.

Thus, the UCL lead author sought to exploit the speed and homogeneity of continuous hydrothermal processes for devising modified flow reactors in which combinatorial ceramic libraries could be created in series. There were several important criteria for the design of any such flow reactor systems, which can generate libraries in series in this way.

(i) A continuous hydrothermal reactor needs to be modified to allow multiple compositions to be introduced, (ii) Strategies for minimizing cross-contamination are required, (iii) Metal salt mixtures need to be accurately mixed manually or via an automated system 
(iv)metal ion distribution in precursors needs to be homogenous (i.e. ideally from solution not a heterogeneous suspension), and

(v) ideally the materials could either be made directly from the process or via a single heat-treatment step post drying (akin to a solid state reaction from nanoparticles ${ }^{91}$ ).

To date, a number of HiTCH flow synthesis (materials discovery) processes have been developed by the UCL authors in the past and are described in numerous publications. $33-34,93,218,267,471,559,648$ The first incarnation of the HiTCH flow synthesis type reactor used a manual injection loop in which metal salts solution of $2 \mathrm{~mL}$ in volume could be sequentially introduced into the flow reactor. In the first report using a $\mathrm{HiTCH}$ flow reactor, over 66 samples were made in a short time (a complete phase diagram of a three-component system) with each sample being separated in steps where samples differed in composition by ca. 10 at\%.

The samples were made in quantities of < $1 \mathrm{~g}$ per sample within a day or so, which would normally take many weeks of synthesis in batch or similar processes. Such small amounts of material adequate as long as the nanomaterials can then be tested for pertinent properties. The same approach can of course be used to generate fewer samples, with each sample being made in larger amounts (if so needed by the testing process). Thus, much of the $\mathrm{HiTCH}$ flow synthesis productivity depends on the reactors design, and the time needed to clean out the reactor between serial samples.

The later designs of the HiTCH flow reactors, the authors added an isco pump to the inlet of the injector (as well as a few minor changes) to allow larger volumes of metal salt precursors to be added, and the third design was a fully automated robot called RAMSI (Rapid Automated Materials Synthesis Instrument). ${ }^{218,471,542}$ The main aspects of the RAMSI system were automated metal mixing, pumping of the mixtures into the reactor and collection and automated clean-up and printing of the ceramic slurries. The process of building automation led to several valuable learning outcomes and approaches, which the authors feel are important for the future. These include the outcome that some parts of high throughput research such as metal salt mixing should be automated, whilst other parts are best done manually!

In 2011 the RAMSI process was decommissioned to make way for a higher throughput manual HiTCH processes that allows more flexible sample sizes.93, 121, 559 Metal salt mixing in the UCL authors laboratory is now conducted by a liquid handler which offers significant time savings and better accuracy than a person doing such tasks. For exam- 
ple, a Hitch type approach was used to make libraries of $\mathrm{Li}$ ion cathode materials based on $\mathrm{LiMn}_{1-x-\mathrm{y}} \mathrm{Fe}_{\mathrm{x}} \mathrm{V}_{\mathrm{y}} \mathrm{PO}_{4} \cdot 559$ Figure 43a shows the cycling performance of the Fe-doped LMP family of cathode materials at different $\mathrm{C}$ rates. Figure $43 \mathrm{~b}$ shows the overall phase diagram of $\mathrm{LiMn}_{1-x-y} \mathrm{Fe}_{x} \mathrm{~V}_{y} \mathrm{PO}_{4 \prime}$ with the area for investigation highlighted with a green triangle, which apply to figure $43 \mathrm{C}$ and $43 \mathrm{~d}$ heat-maps. Heat-maps in the Mn-rich part of the phase diagram show low specific capacity and as expected, the material with the most Fe was amongst the best performing (due to improved electronic conductivity). ${ }^{559}$ A gradual change in properties was observed that suggests that the flow synthesis process allowed access to consistent nanomaterials that were subtly different.

In the current incarnation of HiTCH flow reactors in the laboratory of the UCL authors, typically it is possible to make 66 samples (of ca. $1 \mathrm{~g}$ each) per day, or less samples in larger amounts (e.g. 25 samples in the $10 \mathrm{~g}$ scale per sample in a few hours). High throughput continuous hydrothermal mode has also been carried out using a pilot scale reactor the authors in a similar way, albeit at much higher flow rates (this can give 20 samples at ca. $50 \mathrm{~g} \mathrm{a}$ sample in a few hours typically).

When samples are made using high throughput methods, invariably more rapid methods of gathering useful analytical data required such as automated XRD auto samplers to save time in changing samples. Furthermore, the authors learned that affixing samples into well-plates for automated analysis, was a faster approach to materials screening for nanoceramics, which can be useful to get a basic idea of phase behaviors without having to carry out one at a time slow individual XRD, Raman, UV-Vis etc. or other measurements.

In summary, the nanoparticle discovery approach using continuous hydrothermal flow reactors followed by rapid data collection and analysis, has enabled materials with useful properties to be more quickly identified so that they can then be scaled up using a pilot plant (or larger synthesis process), which will ultimately aid translation up the technology readiness levels, ${ }^{649}$ towards commercial development.

\subsection{Scale-up of Hydrothermal Flow}

Scaling up hydrothermal reactions can pose several new problems and has to be compared to product quality and cost versus other existing technologies which have been commercialized or are based in chemical companies. ${ }^{650}$ Any scaled up hydrothermal process would have to compete with other NP processes such as flame synthesis and plasma synthesis methods. .1-43 $^{1-4}$ 
Hydrothermal reactors have been long since scaled up for batch processes and to some extend this is an area where flow reactors can have a further advantage as they can go to high pressures without compromising safety. In contrast, as batch reactors are scaled up, the require ever thicker walls to achieve high pressures. Hence, scaled up batch reactors might be expected to operate at lower temperatures and pressures compared to an analogous flow system. Having very large batch volumes of superheated fluids also causes a significant hazard to operator health in the case of catastrophic failure due to the large volumes of fluids, which will exist at any one time. Furthermore, getting a homogenous reactor environment inside a scale-up batch reactor becomes very difficult. In contrast, for a scaled up flow process (e.g. x20 from a lab-scale), the actual in pipe mixing "reactors" for hot and cold feeds will only be expected to be slightly larger than the laboratory size. ${ }^{170}$ This is because scaled up continuous hydrothermal reactors can run at a very high flow rates and concentrations as long as the resulting slurry properties are adequate for the process.

Larger scale supercritical water flow systems have been known for some years, particularly for oxidation of chemicals or undesirable wastes. ${ }^{651-652}$ These include oxidation of pharmaceutical wastes, pulp, and paper mill wastes, bulk VX nerve agent hydrolysates, chemical agent, explosives, shipboard wastes (U.S. Navy), rocket, propellant (U.S. Army Force), dyes; chemical agent and explosives (U.S. Army), oxygenated and nitrogen-containing hydrocarbon (e.g., alcohols, glycols, amines). Other oxidation reactions include recovery of spent catalyst (recover platinum group metals ${ }^{653}$ ), oxidation of municipal sludges, PCBs, chlorinated wastes and industrial wastes (e.g. wastewater from a DNT/MNT plant and melamine plants. ${ }^{651}$ The authors are aware that corrosion is certainly a major issue in some cases. For example, in the recovery of precious metals $\mathrm{s}^{60}$ where halide may be present in the feeds, that might give significant corrosion problems at certain temperatures / conditions.

A number of other processes in under high-pressure conditions may also have potential for future scale up in flow if they can me made viable. These include the conversion, treatment or breakdown of "biomass" or natural materials to high value chemicals, e.g. direct liquefaction of biomass (biopolymers cellulose, hemicellulose and lignin) ${ }^{654}$ An excellent review by Loppinet-Serani and colleagues was also published in $2009{ }^{655}$ which covers state-ofthe-art processes using sc- $\mathrm{H}_{2} \mathrm{O}$ (and in some cases alcohols) in the field of environment remediation.

The same authors highlighted the properties of sc-water in sc-Water Oxidation (SCWO), Supercritical water Biomass Gasification (SCBG); and Hydrolysis of Polymers in Supercritical Water (HPSCW) for composites/plastics recycling. ${ }^{655}$ In these applications, sc-water can play the role of a solvent or reagent for organics, polymers, and plas- 
tics to give products including gases, high-value monomers, aqueous and/or organic liquids and energy. For further reading on conversion of biomass in sc-water/subcritical fluids, the author's recommend a review by Toor et al. on "hydrothermal liquefaction of biomass (sub-critical water)" ${ }^{656}$ and a book chapter review by Aymonier et al. on "hydrolysis in near- and supercritical water for biomass conversion and material recycling" .657 Knez et al. have also reviewed a number of high pressure technologies involving sub and Supercritical Fluids that offer the possibility to obtain new sustainable products. ${ }^{658}$ Thus, the role of Supercritical Fluids in forming high value carbon based chemicals is being realized, for example, see a review of the prospects of Supercritical Fluids in realizing graphene-based functional materials ${ }^{621}$ ) as well as the first batch supercritical fluid synthesis of aspect ratio controlled graphenic materials from coal. ${ }^{621}$

To the best of the author's knowledge, other than the Hanwha Corporation (Figure 53), there are relatively few proven large-scale industrial continuous hydrothermal processes for controlled inorganic NP production. The process was first developed to pilot scale before the industrial process was built at an initial capacity of 300 tpa capacity and more recently now this process has been quoted to have the potential to make 1000 tpa). ${ }^{77}$ The existence of lab and pilot plants are known and the UK has two groups, namely at Nottingham and UCL (the authors) London. The Nottingham group has built a prototype pilot plant (using their patented counter-current mixer) which is estimated to have a capacity of less than $10 \mathrm{~kg}$ per day typically, with a larger facility now completed in 2016.372 The authors have also mentioned commercial scale reactors in ITEC Itd Japan. ${ }^{629}$ It is likely that other large scale processes $>2 \mathrm{~kg} \cdot \mathrm{h}^{-1}$, will be developed in the future. Now that various groups around the world have delivered scale-up facilities, results from such processes are beginning to emerge. $32,36,93,120,326,384,430$

Figure 53

A major issue with using any such reactors for many different potential products on any one week is the problem of maintenance of the reactor and systems and also ensuring there is no contamination or corrosion between the productions of samples when the chemistries and $\mathrm{pH}$ regimes can vary considerably. This is important for any high value nanomaterials products where sometimes, minute contamination levels can be crucial. The authors developed a large pilot plant (designed for >3 tpa) as part of an initial UK Engineering and Physical Sciences Research Council (EPSRC) funded academic research project. ${ }^{271}$ This project was designed with the aim of scaling up existing labora- 
tory scale continuous hydrothermal reactors with a significant increase in capacity $32,120,170,261,586,659$ and to address industry need for the commercial production of nanoparticulate products, i.e. consistency, repeatability, predictability and quality assurance. The UCL authors were particularly keen to gather useful knowledge about how mixing occurs on new and existing laboratory and mini-pilot plant scale mixers. It was our belief that by understanding how well mixing occurs between the hot water and a second cold feed (aqueous metal salt solution), it would be possible to guarantee high rates of nucleation and smaller particles. Questions in this work included the following;

(i) What is the quality of products on the lab and pilot plant scales?

(ii) What mixing designs will achieve good and consistent product quality on a large scale?

(iii) What are the environmental concerns with this technology and how can they be solved on the industrial scale?

(iv) How can large volumes of slurries be processed in an acceptable manner via knowledge of colloids science?

(v) What are the maintenance issues for a mini-pilot plant? and

(vi) How can mixing in situ be used to develop a new, improved and patentable mixer which does not infringe on existing IP?

In the latter question, ideally such a mixer should achieve the theoretical mixing of the superheated water and col feed instantaneously or very close to the point of mixing and the products should be carried away from the reaction zone as quickly as possible to avoid buildup of materials.

For the pilot scale CHFS developed by the authors, a confined jet (co-current) mixer was developed that shows excellent scalability. The pilot plant process has now been demonstrated in a number of applications, showing properties or high-performance that is on a par with the lab scale analogous process. ${ }^{32,120,261,558,586,659}$ This suggests that for a range of nanomaterials syntheses, the differing scales of mixers can still retain high Reynolds numbers and therefore a nucleation dominated reaction environment inside the mixer.

The UCL pilot plant was commissioned using a model system of $\mathrm{ZnO}$ NPs. ZnO is ideal for evaluating scale-up processes because the chemistry is very sensitive to changes in nucleation and growth conditions. In addition, due to the relatively high process flow rates used (unlike the comparable laboratory scale processes), the authors were able to study the effects of increasing $\mathrm{Zn}$ precursor concentration (and using a single high $\mathrm{H}_{2} \mathrm{O}_{2}$ concentration) on the growth of the as-prepared $\mathrm{ZnO}$ nanomaterials. The results, showed unexpected and large variation in shape 
and size, could be achieved. ${ }^{120}$ A simplified schematic representation of the continuous hcontinuous hydrothermal synthesisydrothermal synthesis pilot plant is shown in figure 54 and essentially is a 5 times scale up (min) of the current analogous lab CHFS process.

Figure 54

\subsection{Environmental Considerations}

As environmental and related matters receive ever increasing importance, the authours feel that it is only a matter of time before continuous hydrothermal methods will become feasible for nanomaterials manufacture (subject to full Life Cycle Assessments, LCAs to consider energy, consumables and waste usage). For many years, researchers have suggested that continuous hydrothermal flow reactors may offer some benefits in terms of sustainability (as well as the benefits of being able to make high quality nanomaterials). In the first reported study of its kind, Sonnemann et al. reported the potential environmental impacts leading from the lab-scale supercritical synthesis of $1 \mathrm{~kg}$ of $\mathrm{Ba}_{0.6} \mathrm{Sr}_{0.4} \mathrm{TiO}_{3}$ nanoparticles (in alcohol-water mixtures) by reporting an anticipatory life-cycle assessment. ${ }^{660} \mathrm{The}$ researchers, conducted a "cradle-to-gate" assessment, estimating the impacts across the various steps involved, from resource extraction, material processing, and finally the continuous synthesis of the nanoparticles (end-of-life considerations were not investigated in this case). The study was useful because it suggest a number of ways by which the environmental profile/footprint of the supercritical synthesis process could be improved. ${ }^{660}$ Importantly, being able to synthesize nanoparticles with precursor concentrations of 0.1 and 1.0 molar was shown to reduce average life-cycle impacts by ca. 81 and $95 \%$, respectively. Furthermore, the high rates of recovery / reuse of solvents, could further reduce average life-cycle impacts (by $56 \%$ ). Further savings were possible by replacing the alkoxides for acetates or hydroxides (latter for barium and strontium). ${ }^{660}$ Further research in the future should be extended to cover the full cradle-to-grave LCA, including the use and end-of-life stages in order to have an even more accurate holistic view of the process benefits.

\section{FUTURE DIRECTIONS AND CAPABILITIES}

Summary: The design of continuous hydrothermal reactor systems and types of experiments that can be carried out, are evolving year upon year and it appears that several themes are emerging area that are hopefully covered in 
this review. The authors note that this review has been broken down by applications of materials, but actual evaluation in that specific application was not always demonstrated. For example in subjects such as optical applications for UV attenuating nanoparticles (made via CHFS), the properties have been well explored and whilst there are still regulations for such materials such as in suncreams, such materials could be readily commercialized if the business case and performance metrics can be met. Such materials would be at the higher cost end of the market. In optical applications such as refractive index modifiers made via CHFS methods, there is still much work to be done in order to make these materials useful in the market; first they need to be well dispersed in solvents and secondly, they need to be fully evaluated. If this can be done in the future at a reasonable cost, then there are a number of markets, e.g. automotive or protective coatings / paints markets, where such materials can add value. Nano-pigments made via CHFS will most likely have use in the future in formulations where they can be printed such as inkjet or when added to polymers to afford colour and rigidity. Thermochromics possess switching properties at various temperatures so if they are to find applications, it may be that their deposition or incorporation into glass coatings or devices for solar heat control will need further development, e.g. it may be possible to coat NPs via aerosol methods onto glass during glass manufacture. In nanoparticles made via CHFS for healthcare (nanophosphors, bioceramics, antimicrobials magnetic), the authors suggest these materials will not rapidly reach the market due to the regulatory barriers (often requiring ppm level control of impurities), established products and general high costs to developing therapeutic nanoparticles in general.

Amongst the areas where much research and testing has been carried out, is in the area of nanoparticles for electronics, sensors, Information and communication technologies (metals \& transparent conducting oxides, semiconductors for electronics or gas sensors, dielectrics, piezoelectrics, ferroelectrics, multiferroics, magnetic particles). In these areas, whilst the number of nanoparticles have been made, testing and evaluation of such materials is usually carried out for one particular application, however it is known that some of these materials have multiple applications. Therefore, in the future, it may be vital that nanomaterials are screened for multiple uses as they may not be optimum for one application and much better for another. In particular, the development of printable methods (e.g. screen print or inkjet, 3D printing followed by suitable post deposition treatments, which can exploit lower sintering temperatures) to allow the assembly of complex patterns for devices, may hold the key to implementation of nanomaterials for electronic device applications. 
To date a number of nanomaterials made via CHFS methods, have been evaluated in the areas of photocatalysis as well as gas phase heterogeneous catalysis. In the area of photocatalysis; whilst nanomaterials offer significant higher BET surface areas, they are often so small that electron hole recombination may dominate and lead to poor photocatalytic activity. Therefore, in this case it may be often preferable to sacrifice some surface area and grow particles slightly larger in order to reduce recombination and enhance overall photoactivity. In heterogeneous catalysis a significant number of catalytic test studies have been carried out on particles made in continuous hydrothermal reactors). The authors suggest that the development of new heterogeneous catalysts for cleaning emissions from automotive vehicles or polluting industries is still underdeveloped area with tremendous benefit to mankind. Furthermore, there is a need to develop more complex heterogeneous catalysts mixtures or agglomerates that display reduced sintering upon ageing. Another area where nanomaterials made in flow reactors have not really been tested are in very important processes such as Fischer-Tropsch reactions of forming hydrocarbons or highly energy intensive processes such as the formation of ammonia, where energy savings might be possible as a result of reductions in energy. In Fischer-Tropsch catalysts, the smaller size can offer greater stability to oxidative cycling to regenerate catalysts, however to date, there has still not been a single report of a CHFS made nanomaterial being tested for such applications yet.

The area of energy harvesting and energy conversion or oxygen/fuel generation devices, from nanomaterials made in flow reactors, is now developing apace. Prototype devices and membranes in the fields of solid oxide fuel cells and oxygen separation membranes and now being tested, and in the latter case is being published. ${ }^{544}$ At the time of writing this review, there has only been one report on testing of solid oxide fuel cells that incorporated CHFS-made nanoparticles (symmetrical cells).537 In oxygen separation membranes whilst nanocomposite materials incorporating CHFS made nanoparticles with low precious metal contents, some work is still required to make the membranes thinner and more robust, before they can reach commercial applications

In the field of thermoelectrics and energy scavenging devices (such as from piezoelectric's) nanoparticles made in CHFS reactors have never been evaluated to the authors knowledge, so in device testing and evaluation is still required; in the case of thermoelectrics, use of rapid sintering techniques to give nanograined composites, may also be beneficial to made devices. Furthermore, it is known that many of the materials that perform poorly as transparent conducting oxides, often display interesting thermoelectric properties. Therefore in the future the authors suggest that the features of be tested various applications where possible. 
Perhaps one of the most exciting areas for nanomaterials made via CHFS type processes has been in the field of energy storage, specifically lithium ion rechargeable batteries. To date, a number of papers have been published in witch half-cells of anode and cathode materials have been evaluated and shown from excellent to performance, particularly at high power. Only recently has the first full Li-ion cell been reported for the case where at least one of the materials was made via CHFS. In contrast with Li-ion batteries, there are still no reports to the author's knowledge on CHFS materials being used to make supercapacitors, lithium air or zinc air, or sodium ion batteries or indeed multivalent batteries. Given the importance of such devices in energy storage applications, it is inevitable that before too long researchers in CHFS will explore these key application areas for energy storage.

The authors feel that there are a number of opportunities for continuous hydrothermal processes and nanomaterials made from continuous hydrothermal which will be developed in the future, which include the following;

1. Exploring materials synthesis at lower temperature and for longer times (i.e. operating at lower temperatures, e.g. in the range 200 to $250^{\circ} \mathrm{C}$ for longer, i.e. multi-minutes not a few seconds residence times)

2. Use of more atom efficient processes and heat recovery methods for more sustainable nano-manufacturing (particularly for scale-up)

3. The development of NPs with improved catalytic performance / lifetime of catalysts after accelerated ageing.

4. Use of novel engineering solutions to complex multilayered (e.g. core-shell) or other particles, particularly in the area of organic coatings. ${ }^{158}$

5. "Higher throughput" continuous hydrothermal synthesis (i.e. number of samples) to exploit the speed of the method and develop new materials which are more sustainable and rely less on rare and expensive metals (for example via a materials discovery approach).

6. Design of novel continuous hydrothermal apparatus, which can allow in situ investigations into nucleation and growth processes.

7. Development of microreactors, ${ }^{172,}{ }^{661}$ which allow parallel synthesis or extreme conditions or reagents, which would not be safer at higher scales.

8. In situ measurements (X-ray based such as methods developed by Iversen and coworkers) and imaging (e.g. neutron imaging ${ }^{161-162,662}$ ) to better understand mixing dynamics in different mixers. 
9. Better use of lifecycle analyses ${ }^{660}$ in assessing routes to commercial development; includes directly formulating the products from the reactor with minimal downstream processing. Ideally such studies would include the full cradle-to-grave LCA to have a more accurate holistic view (i.e. include the stages in use and for end of life)

10. Rapid test methods or automated probes for testing nanoceramics in order to build structure, propertycomposition relationships.

11. Safe methods that allow NPs to be coated onto substrates with good adhesion whilst maintaining small particle size.

12. Develop methods to consolidate nanoceramics for the manufacture of dense disks or devices or nanostructured (or hybrid) composites.

13. A greater role for continuous hydrothermal syntheses in the development of nanoceramics incorporating carbons such as graphenes for a range of applications such as energy storage or catalysis (note: the role of SCFs in processing of graphene-based materials has been reviewed by Aymonier et al. ${ }^{621}$ ).

14. Develop better and scalable NP recovery strategies especially at large scale (in flow membrane technologies).

15. Develop cleaner particles for biomedical use that can meet regulatory hurdles..$^{22}$

16. Developments in catalysis to explore the effects of NP size (e.g. rounded particles rather than faceted ${ }^{663}$ ), or shape (such as faceted $\mathrm{Cr}$-doped ceria) ${ }^{664}$ or doped activating metals ${ }^{665}$ can improve stability and performance of catalysts.

17. Gaining a better understanding of how temperature and other process conditions are linked ${ }^{666}$ and affect the products obtained.

18. New precursors or additives, for access to novel materials or precise control of particle properties such as change redox states (e.g. making hematite rather than magnetite ${ }^{667}$ ) or size / shape control.

19. The extension of modeling (e.g. CFD) and design of mixing arrangements (e.g. confined jet mixer followed by a counter-flow mixer in the second stage ${ }^{36,668}$ ) to improve product outcomes, get more consistent product quality, higher temperatures, longer residence times (e.g. $>10$ minutes as for the synthesis of tetragonal $\mathrm{SnO}_{2}{ }^{669}$ ) and reduce blockages.

20. Develop functionalized NPs (so NP can be dispersed into hydrophobic or hydrophilic solvents ${ }^{670}$ ) for inks to allow large-scale devices to be printed at lower costs. 
21. Investigation of the benefits of post-synthesis annealing or flash heating to preserve particle attributes whilst improving crystal quality.

22. looking at the opportunities for low temperature metastable phases

23. Develop materials with structural hierarchy or biomimetics. ${ }^{625,523}$

24. Use flow reactors for removing toxic or radioactive metals from liquids ${ }^{74}$ or for reclaiming valuable inorganic elements from waste streams or creating value from waste (including synergistic effects of organics in inorganic waste for optimized effect, e.g. decomposing metal complexes from electroplating waste). ${ }^{77,521,667}$

25. Developing reactions that can give direct access to complex phases (e.g. heterometallic oxides ${ }^{671}$ ), preferably with high surface areas that are unobtainable by low temperature or batch processes.

26. Scale-up of laboratory processes to get consistent product quality as that achieved on a laboratory scale (see publication from the authors on Ce-Zn oxides ${ }^{524}$ for which pilot plant was run at but 17.5 times total flow rate of lab scale process with indiscernible particle properties) or at least good enough to be able to exploit in a commercial application.

Clearly, some of the above emerging themes will be important in the continued development of continuous hydrothermal processes as they reach more widespread adoption amongst not only the materials science community, but also industry.

\section{CONCLUSIONS AND OUTLOOK}

The use of continuous hydrothermal flow synthesis systems as first pioneered by Japanese and USA researchers, has come a long way from the initial studies of particle crystallization with changing reactor parameters). The many advantages of making NPs via continuous hydrothermal technology are apparent. Materials can be made with extremely high surface areas, high sinterabilities and crystallinities, which will drive a future revolution in low temperature sintering for new devices and nanograined materials / coatings ${ }^{672}$ particularly in the energy sector (energy storage, transmission and absorption). The number of applications for such nanoceramics is unlimited and through materials discovery approaches and rapid screening, we will be able to develop materials faster for scale up and market readiness. Indeed, the fact that NPs are made in an aqueous environment is definitely very favorable for both indus- 
try (e.g. limiting worker exposure to potentially harmful particles) and for potential applications were particle agglomeration is to be avoided (e.g. such as formulation of printable ceramic inks).

In recent years, researchers have begun to look at not only a variety of different mixing and reactive approaches to control particle properties, but also into the role of additive and surfactants in order to control the redox chemistry and particle growth and agglomeration including in hierarchical nanomaterials. New additives are still in need to be evaluated for NPs in order to allow them to be recovered more readily for example from the water or to be eventually dispersed in a polymer or organic solvent base. The surfactants must be robust enough to survive the CHFS process and also possess suitable properties, e.g. to not bridge particles together (otherwise gelation will result); such surfactants will be developed by a process of trial and error initially as many of the known properties towards larger colloids will no longer apply, and therefore, contributions to this field are required.

The next decade or so will see the exploitation by researchers of the favorable properties of nanomaterials made using continuous methods such as the continuous hydrothermal technique. In the development of more elaborate reactor designs as possible to build up multifunctional (or smart) particles in a single system or via particle modification. For example at the writing of this review, new dual stage reactors reports were published to develop "coreshell" or coated NPs of $\mathrm{SnO}_{2}$ on titania. ${ }^{158}$ Such is the flexibility of continuous hydrothermal systems will increase reproducibility despite the added complexity of the NPs.

Scale-up (or scale out) of the continuous hydrothermal flow reactors is being undertaken in order to produce meaningful quantities of NPs that will allow industrial and academic end users to evaluate the advantages of the technology. The important challenges in scale-up will be to ensure that the quality of the products produced on the larger scales, will be comparable or better to those produced on the laboratory scale and that the system can be safely operated. This route not only affords huge reductions of energy costs and number of steps, which is becoming ever more attractive due to the escalating costs of energy for industry. Researchers will also need more ingenious tools with which to be able to look inside mixers to better understand how to improve mixing. Synchrotron techniques such as neutron imaging. ${ }^{162,662}$ are one example of how such insights could be gained as well as tomographic methods.

The continuous hydrothermal method is ideally of use for high throughput nanomaterials synthesis and discovery. Advantageously, this not only exploits the speed of syntheses but also uses the fact that the nano-sized materials from continuous hydrothermal processes, can be highly reactive and in some cases have unprecedented high 
intimacy of mixing between two phases, enabling mass transfer problems to be overcome in "difficult to make" solid-state syntheses. ${ }^{38,91}$ In this way, complex functional ceramics can be generated with less waste and energy input, in a fraction of the time, which will be of interest to both industry and academia.

One cannot simply assume that the current phase diagrams as we know them should be applied to results obtained from continuous hydrothermal routes. There are examples where heterometallic materials are obtained via CHFS with different characteristics to their conventionally made counterparts. This is due to a combination of fast conversion from reagent to product and relatively low temperature of synthesis, that creates an unusual environment, leading to kinetic products. The authors have also observed that as well as kinetic phases, it's also possible to sometimes obtain slightly differing thermodynamic products (e.g. products formed after heat-treating the kinetic

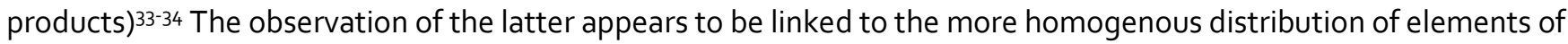
the initial kinetic phases made in flow, which when heat-treated, can have stability or phase purity that is unlike materials made via conventional solid-state methods for example) $)^{33-34}$

The use of computational research and methods will also be key in the development of continuous hydrothermal syntheses. For example, the integration of data mining with synthesis, characterization and testing data, may have a role to play in understanding structure-property-compositional relationships in the future. Through computational fluid dynamics, researchers will be able to develop better mixing approaches on the laboratory and larger scales. Such work is already underway and will be set to expand as the requirements for particle size control become ever more demanding. In line process monitoring will be able useful for quality assurance purposes. Again, there are many studies underway at the moment that will hopefully lead to novel capabilities in the forthcoming years. The application of continuous hydrothermal synthesis technology for producing tailored nanomaterials, composites and bulk or consolidated nanograined ceramics, has a bright future.

In closing, the challenge is for researchers to work in multidisciplinary teams with chemists, materials scientists, electrochemists, device engineers, biologists or medical engineers, coatings specialists, crystallographers, beamline scientists, applications specialists, modelers, physicists and chemical engineers, in order to exploit the useful properties of hydrothermal flow reactors towards real applications and needs of industry. By doing so, there is the opportunity to produce ever more efficient nanomaterials and devices for the benefit of mankind on a scale that has not yet been seen but has long been anticipated. The authors hope to be at the vanguard of that nano- 
technological revolution and we hope that this review will inform those in industry and academia who yet to appreciate the potential that nanomaterials by design can me made via continuous hydrothermal flow processes.

\title{
ACKNOWLEDGEMENTS
}

The authors would like to acknowledge the various contributions made by researcher in the Clean Materials Technology group over the last 16 years in helping to develop research and contributions to this review including Dr Josie Goodall and Dr Paul Boldrin (for early support). More recently Dr Chris Tighe (the excellent Figure 2a) and Dr Rob Gruar to co-develop the pilot plant and confined jet mixers. Others who helped with collating information for the large table and some of the text in sections or proof reading versions include Dr Liam McCafferty, Dustin Bauer, Dr Chris Starkey, Dr Clement Denis, Dougal Howard, Dr Peter Marchand, Dr Marco Bersani, Dr Tom Ashton, Dr Alistair Holdsworth, Kalyani Gupta, Ian Johnson, Charles Footer, Yijie Xu (aka Adrian) and Meggi Lubke.

\author{
ABBREVIATIONS \\ $\mathrm{AC}=$ alternating current \\ AGZO = aluminium and gallium doped zinc oxide \\ AZO = aluminium doped zinc oxide \\ $\mathrm{BET}=$ Brunauer-Emmett-Teller \\ $\mathrm{BPR}=$ back pressure regulator \\ BTC = benzene-1,3,5-tricarboxylic acid \\ $\beta$-TCP = beta tri-calcium phosphate \\ $\mathrm{C}=$ cooler \\ $\mathrm{CAN}=$ cerium ammonium nitrate
}


$\mathrm{CCR}=$ co-current reactor

$\mathrm{CDHA}=$ calcium deficient hydroxyapatite

$\mathrm{CGO}=$ ceria-gadolinia

$\mathrm{CHA}=$ carbonated hydroxyapatite

CHFS = continuous hydrothermal flow synthesis

$\mathrm{CHO}=$ Chinese hamster ovary

$\mathrm{COS}_{7}=$ Fibrobrast-like cell line of $\mathrm{CV}-1$ (simian) origin, carrying the $\mathrm{SV}_{40}$ genetic material

$\mathrm{CRT}=$ cathode ray tube

$\mathrm{CSO}=$ ceria - samaria

CTO = calcia-titania

$C V D=$ chemical vapour deposition

$\mathrm{CWO}=$ catalytic wet oxidation

$C Z$ = ceria-zirconia

$C Z A=$ alumina-supported ceria-zirconia

D50 = cumulative $50 \%$ point of diameter

$D C=$ direct current

$\mathrm{DHCA}=3$ 4-dihydroxyhydrocinnamic acid

DLS = dynamic light scattering

DNA = deoxyribonucleic acid

DSSC $=$ dye sensitized solar cell

$E=$ potential

E. coli $=$ escherichia coli

$\varepsilon^{\prime}=$ dielectric constant

$\varepsilon_{\mathrm{r}}=$ relative permittivity

$E_{a}=$ activation energy

$\mathrm{EC}=$ electrochemical capacitors

EDC = 1-ethyl-3-(dimethylaminopropyl) carbodiimide hydrochloride

$E D L C=$ electrochemical double-layer capacitor 
EDS = energy dispersive $\mathrm{X}$-ray spectroscopy

EDTA $=$ Ethylene diamine tetra-acetic acid

EXAFS $=$ extended $X$-ray absorption fine structure

FDA = food and drug administration

FED = field emission displays

FFT $=$ fast Fourier transform

FT = Fischer-Tropsch

FTIR = Fourier transform infrared

FTO = fluorine-doped tin oxide

$\mathrm{GC}=$ gas chromatography

$\mathrm{GZO}=$ gallium doped zinc oxide

$\mathrm{h}^{+}=$hole

$\mathrm{HA}=$ hydroxyapatite

HAADF = high-angle annular dark-field

HAls = hospital-acquired infections

$\mathrm{HiTCH}=$ high throughput continuous hydrothermal

HPLC = high pressure liquid chromatography

HT-continuous hydrothermal = high throughput-continuous hydrothermal

$\mathrm{I}=$ current

$\mathrm{IL}-12=$ Interleukin 12

$\mathrm{IR}=$ infrared

ISHA = International solvothermal and hydrothermal association

ITO = tin-doped indium oxide

IW $=$ incipient wetness

JCPDS = joint committee on powder diffraction standards

$\mathrm{k}=$ thermal conductivity

$k^{\prime}=$ composite rate constant

$\mathrm{KTO}=$ potassium hexatitanate $\left(\mathrm{K}_{2} \mathrm{O} .6 \mathrm{TiO}_{2}\right)$ 
$\mathrm{K}_{\mathrm{w}}=$ equilibrium constant for water

$\mathrm{LCO}=$ lithium cobalt oxide

LEDs = light emitting diodes

LFP = lithium iron phosphate

LMFP= lithium manganese iron phosphate

LMO = lithium manganese oxide

LMP = lithium manganese phosphate

LSCF = lanthanum strontium cobalt ferrite

$\mathrm{LTO}=$ lithium titanate

LUSI = London university search instrument

$M(T)$ = magnetization-temperature curve

$\mathrm{MB}=$ methylene blue

$\mathrm{MDH}=$ magnesium di-hydroxide

$\mathrm{MI}$ = magnetoimpedance

MIECs = mixed ionic-electronic conductors

MNPs = magnetic nanoparticles

MOF = metal-organic framework

$\mathrm{MP}=$ mixing Point

$M R I=$ magnetic resonance imaging

MST = metal-to-semiconductor transition

nc- = near-critical

NCA = nickel cobalt aluminum oxide

NHS = National Health Service

NIB = sodium ion battery

NMR = nuclear magnetic resonance

$\mathrm{OER}=$ oxygen evolution reaction

ORR = oxygen reduction reaction

$\mathrm{OSC}=$ oxygen storage capacity 
$\mathrm{P}=$ pump

$\mathrm{P}_{25}=$ commercial mixture of anatase and rutile structured $\mathrm{TiO}_{2}$

$P_{53}=$ tumour protein 53

PDP = plasma displays

PDF = pair distribution function

PEMFC $=$ polymer electrolyte membrane fuel cell

$P G=$ pressure gauge

$\mathrm{pK}_{\mathrm{a}}=$ acid dissociation constant

$\mathrm{pK}_{\mathrm{w}}=$ negative log of equilibrium constant for water

$\mathrm{PL}=$ photoluminescence

PLD = pulsed laser deposition

PVA = polyvinyl alcohol

PVP = polyvinylpyrrolidone

PXRD = powder $X$-ray diffraction

$\mathrm{Q}=$ quality factor (where $\mathrm{Q} \approx 1 / \tan \mathrm{d}$ )

$\mathrm{Q}$ dots = quantum dots

RAMSI = rapid automated materials synthesis instrument

RESS = rapid expansion of supercritical solution

$\mathrm{RI}=$ refractive index

$\mathrm{ROR}=$ reduction-oxidation-reduction

ros $=$ reactive oxygen species

SAXS = small-angle $X$-ray scattering

sc- = supercritical

$\mathrm{SCR}=$ selective catalytic reduction

SEI = solid electrolyte interphase

SEM = scanning electron microscope

SFN = surface-functionalized NP

SHE = standard hydrogen electrode 
SHP = supercritical hydrothermal process

SMSI = strong-metal support interaction

SOFC $=$ solid oxide fuel cell

SPR = surface plasmon resonance

SPS = spark plasma sintering

SuPS = suspension plasma spraying

STEM = scanning transmission electron microscopy

SV= switch valve

Syngas $=$ synthesis gas

TÆDDI = tomographic angle and energy dispersive diffraction imaging

$\tan \delta=$ dielectric loss

$\mathrm{TC}=$ thermocouple

TCOs = transparent Conducting Oxides

$\mathrm{TE}=$ thermoelectric

TEDDI = tomographic energy dispersive diffraction imaging

TEM = transmission electron microscope

TiBALD = Titanium(IV) bis(ammonium lactato)dihydroxide

TNF-alpha = tumor necrosis factor alpha

TPD = temperature programmed desorption

TPR = temperature programmed reduction

TWC $=$ three-way catalyst

UV = ultraviolet

$\mathrm{VO}_{2}-\mathrm{R}=$ rutile structured vanadium(IV) dioxide

VOC $=$ volatile organic compound

WAXS $=$ wide-angle $X$-ray scattering

XANES = X-ray absorption near edge structure

$\mathrm{XPS}=\mathrm{X}$-ray photoelectron spectroscopy

$\mathrm{XRD}=\mathrm{X}$-ray diffraction 
YAG = yttrium aluminum garnet

YAM = ytrrium aluminum monoclinic

YAP = ytrrium aluminum perovskite

YSZ = yttira stabilized zirconia

BIOGRAPHIES

REFERENCES

1. Kagan, C. R.; Murray, C. B., Charge transport in strongly coupled quantum dot solids. Nature Nanotech. 2015, 10, 1013-1026.

2. Scher, J. A.; Elward, J. M.; Chakraborty, A., Shape Matters: Effect of 1D, 2D, and 3D Isovolumetric quantum confinement in semiconductor nanoparticies. J. Phys. Chem. C 2016, 120, 24999-25009.

3. Rai, S.; Rai, A., Review: Nanotech. - The secret of fifth industrial revolution and the future of next generation. Nus. Biosci. 2015, 7, 61-66.

4. Fujishima, A.; Zhang, X., Titanium dioxide photocatalysis: present situation and future approaches. Com. Ren. Chim. 2006, 9, 750-76o.

5. Pilkington commercial self-cleaning glass; see website http://www.pilkington.com/international+products/activ/.

6. Galkin, A. A.; Turakulova, A. O.; Lunin, V. V.; Grams, J., The oxidation of CO and adsorption of hydrogen on nanocrystalline catalysts of the composition $\mathrm{Pd} / \mathrm{ZrO}_{2}\left(\mathrm{TiO}_{2}\right)$ prepared in sub- and supercritical water. Russ. J. Phys. Chem. 2005, 79, 881-885.

7. Muhammad, I. D.; Awang, M.; Shaari, K. Z. B. K., Potentials of nano catalysts for energy reduction during synthesis of ammonia. IJEIR 2014, 3, 904 - 908 
8. Acar, C.; Dincer, I.; Naterer, G. F., Review of photocatalytic water-splitting methods for sustainable hydrogen production. Int. J. Energy Res. 2016, 40, 1449-1473.

9. Sharma, N.; Ojha, H.; Bharadwaj, A.; Pathakc, D. P.; Sharma, R. K., Preparation and catalytic applications of nanomaterials: a review. RSC Adv. 2015, 5, 53381-53403

10. Bera, A.; Belhaj, H., Application of Nanotech. by means of nanoparticles and nanodispersions in oil recovery - A comprehensive review. J. Nat. Gas Sci. Eng. 2016, 34, 1284-1309.

11. Ren, X.; Han, D.; Chen, D.; Tang, F., Large-scale synthesis of hexagonal cone-shaped ZnO nanoparticles with a simple route and their application to photocatalytic degradation. Mater. Res. Bull. 2007, 42, 807-813.

12. Wang, H.; Xie, C.; Zhang, W.; Cai, S.; Yang, Z.; Gui, Y., Comparison of dye degradation efficiency using ZnO powders with various size scales. J. Hazard Mater. 2007, 141, 645-652.

13. Chen, X. B.; Mao, S. S., Synthesis of titanium dioxide $\left(\mathrm{TiO}_{2}\right)$ nanomaterials. J. Nanosci. Nanotech. $2006,6,906-925$

14. Al Salim, N. I.; Bagshaw, S. A.; Bittar, A.; Kemmitt, T.; McQuillan, A. J.; Mills, A. M.; Ryan, M. J., Characterisation and activity of sol-gel-prepared $\mathrm{TiO}_{2}$ photocatalysts modified with $\mathrm{Ca}$, Sr or $\mathrm{Ba}$ ion additives. J. Mater. Chem. 2000, 10, 2358-2363.

15. Akihiko, K.; Yugo, Y., Heterogeneous photocatalyst materials for water splitting. Chem. Soc. Rev. $2008,38,253-278$.

16. Anpo, M.; Takeuchi, M., The design and development of highly reactive titanium oxide photocatalysts operating under visible light irradiation. J. Catal. 2003, 216, 505-516.

17. Serpone, N.; Dondi, D.; Albini, A., Inorganic and organic UV filters: Their role and efficacy in sunscreens and suncare products. Inorg. Chim. Acta 2006, 360, 794-802.

18. Müller, D.; Cattaneo, S.; Meier, F.; Welz, R.; de Vries, T.; Portugal-Cohen, M.; Antonio, D. C.; Cascio, C.; Calzolai, L.; Gilliland, D., Inverse supercritical fluid extraction as a sample preparation 
method for the analysis of the nanoparticle content in sunscreen agents. J. Chromatogr. A 2016, $1440,31-36$.

19. Li, X.; Xiang, R.; Su, T.; Qian, Y., Synthesis and electrochemical properties of nanostructured $\mathrm{LiMn}_{2} \mathrm{O}_{4}$ for lithium-ion batteries. Mater. Lett. 2007, 61, 3597-3600.

20. Adschiri, T.; Hakuta, Y.; Kanamura, K.; Arai, K., Continuous production of $\mathrm{LiCoO}_{2}$ fine crystals for lithium batteries by hydrothermal synthesis under supercritical condition. High Press. Res. 2001, $20,373-384$.

21. Hossein-Babaei, F.; Keshmiri, M.; Kakavand, M.; Troczynski, T., A resistive gas sensor based on undoped p-type anatase. Sens. Act. B-Chem. 2005, 110, 28-35.

22. Kumazawa, N.; Islam, M. R.; Takeuchi, M., Photoresponse of a titanium dioxide chemical sensor. J. Electroanal. Chem. 1999, 472, 137-141.

23. UCL, Clean Materials Technology group website (research) see http://chemb125.chem.ucl.ac.uk/Pages/Research.html.

24. Patel, D.; Kellici, S.; Saha, B., Green process engineering as the key to future processes. Processes 2014, 2, 311-332.

25. Adschiri, T.; Byrappa, K., Supercritical hydrothermal synthesis of organicinorganic hybrid nanoparticles. In Nanohybridization of Organic-Inorganic Materials, Muramatsu, A.; Miyashita, T., Eds. Springer: Berlin Heidelberg, 2009; 247-280.

26. Yoshimura, M.; Byrappa, K., Hydrothermal processing of materials: past, present and future. J. Mater. Sci. 2008, 43, 2085-2103.

27. Byrappa, K.; Adschiri, T., Hydrothermal technology for Nanotech.. Prog. Cryst. Growth Charact. Mater. 2007, 53, 117-166.

28. Darr, J. A.; Poliakoff, M., New directions in inorganic and metal-organic coordination chemistry in Supercrit. Fluids. Chem. Rev. 1999, 99, 495-541. 
29. Lubke, M.; Johnson, I.; Makwana, N. M.; Brett, D. J. L.; Shearing, P.; Liu, Z.; Darr, J. A., High power layered titanate nano-sheets as pseudocapacitive lithium-ion battery anodes. J. Power Sources 2016, 305, 115-121

30. Toyama, S.; Hayashi, H.; Takesue, M.; Watanabe, M.; Smith, R. L., Synthesis of alkali niobate $K_{1-}$ ${ }_{x} \mathrm{Na}_{x} \mathrm{NbO}_{3}$ nanoparticles using a supercritical water flow system. The J. Supercrit. Fluids 2016, 107, 1-8.

31. Theodet, M.; Qulifen, C.; Martinez, C.; Aymonier, C., Continuous supercritical synthesis of unsupported and high specific surface area catalyst precursors for deep-hydrodesulfurization. J. Supercrit. Fluids 2016, 117, 252-259.

32. Makwana, N. M.; Tighe, C. J.; Gruar, R. I.; McMillan, P. F.; Darr, J. A., Pilot plant scale continuous hydrothermal synthesis of nano-titania; effect of size on photocatalytic activity. Mater. Sci. Semicond. Proc.. 2016, 42, 131-137.

33. Weng, X. L.; Cockcroft, J. K.; Hyett, G.; Vickers, M.; Boldrin, P.; Tang, C. C.; Thompson, S. P.; Parker, J. E.; Knowles, J. C.; Rehman, I.; Parkin, I.; Evans, J. R. G.; Darr, J. A., High-throughput continuous hydrothermal synthesis of an entire nanoceramic phase diagram. J. Comb. Chem. 2009, 11, 829-834.

34. Quesada-Cabrera, R.; Weng, X.; Hyett, G.; Clark, R. J.; Wang, X. Z.; Darr, J. A., High-throughput continuous hydrothermal synthesis of nanomaterials (part II): unveiling the as-prepared $\mathrm{Ce}_{x} \mathrm{Zr}_{\mathrm{y}} \mathrm{Y}_{\mathrm{z}} \mathrm{O}_{2 \text {-delta }}$ phase diagram. ACS Comb. Sci. 2013, 15, 458-63.

35.http://www.Nanotech..basf.com/group/corporate/Nanotech./en/function/conversions:/publishdow nload/content/microsites/Nanotech./images/dialogue/Final report Dialogforum Nano of BASF 2014_2015.pdf. 
36. Gruar, R. I.; Tighe, C. J.; Southern, P.; Pankhurst, Q. A.; Darr, J. A., A direct and continuous supercritical water process for the synthesis of surface-functionalized nanoparticles. Ind. Eng.

Chem. Res. 2015, 54, 7436-7451.

37. B. W. Robinson, C. J. T., R. I. Gruar, A. Mills, I. P. Parkin, A. K. Tabecki, H. L. de Villiers Lovelock, J. A. Darr Suspension plasma sprayed coatings using dilute hydrothermally produced titania feedstocks for photocatalytic applications. 2015 , 3, 12680-12689.

38. Weng, X.; Boldrin, P.; Abrahams, I.; Skinner, S. J.; Kellici, S.; Darr, J. A., Direct syntheses of $\mathrm{La}_{(n+1)} \mathrm{Ni}_{(n)} \mathrm{O}_{(3 n+1)}$ phases ( $\mathrm{n}=1,2,3$ and infinity) from nanosized co-crystallites. J. Solid State Chem. 2008, 181, 1123-1132.

39. DTI report: Nanomaterials Manufacture and Applications. Available at http://www.oti.globalwatchonline.com/online_pdfs/36433MR.pdf. 2008.

40. Suzuki, K.; Kijima, K., Phase transformation of $\mathrm{BaTiO}_{3}$ nanoparticles synthesized by RF-plasma CVD. J. Alloys Compd. 2006, 419, 234-242.

41. Arutanti, O.; Arif, A. F.; Balgis, R.; Ogi, T.; Okuyama, K.; Iskandar, F., Tailored synthesis of macroporous $\mathrm{Pt} / \mathrm{WO}_{3}$ photocatalyst with nanoaggregates via flame assisted spray pyrolysis. AlChE J. 2016, 62, 3864-3873.

42. Chae, S.; Lee, H.; Pikhitsa, P. V.; Kim, C.; Shin, S.; Kim, D. H.; Choi, M., Synthesis of terraced and spherical MgO nanoparticles using flame metal combustion. Powder Technol. 2017, 305, 132-140.

43. Wang, S. H.; Huang, Y., Flame aerosol synthesis of $\mathrm{WO}_{3} / \mathrm{CeO}_{2}$ from aqueous solution: Two distinct pathways and structure design. Chem. Eng. Sci. 2016, 152, 436-442.

44. Anastas, P. T.; Warner, J. C., Green Chemistry: Theory and Practice. Oxford University Press: New York, 1998.

45. Schlabach, D. V. S. S., Microwave Plasma Synthesis of Materials - From Physics and Chemistry to Nanoparticles: A Materials Scientist's Viewpoint Inorganics 2014, 2, 468-50. 
46. Yokel, R. A.; MacPhail, R. C., Engineered nanomaterials: exposures, hazards, and risk prevention. J. Occu. Med. Tox. 2011, 6:7, 1-27.

47. Danks, A. E.; Hall, S. R.; Schnepp, Z., The evolution of 'sol-gel' chemistry as a technique for materials synthesis. Mater. Horiz. 2016, 3, 91-112.

48. Feinle, A.; Elsaessera, M. S.; Hüsinga, N., Sol-gel synthesis of monolithic materials with hierarchical porosity Chem. Soc. Rev. 2016, 45, 3377-3399.

49. Koźlecki, T.; Polowczyk, I.; Bastrzyk, A.; Sawiński, W., Improved synthesis of nanosized silica in water-in-oil microemulsions. J. Nanopart. 2016, 2016 Article ID 8203260.

50. Roedern, E.; Lee, Y.-S.; Ley, M. B.; Park, K.; Cho, Y. W.; Skibsteda, J.; Jensen, T. R., Solid state synthesis, structural characterization and ionic conductivity of bimetallic alkali-metal yttrium borohydrides $\mathrm{MY}\left(\mathrm{BH}_{4}\right)_{4}(\mathrm{M}=\mathrm{Li}$ and Na) J. Mater. Chem. $A$ 2016, 4, 8793-8802.

51. RSC/Royal Academy of Engineering Report on Nanotech.: Nanoscience and Nanotechnologies; Opportunities and Uncertainties, 2004, Available at http://www.nanotec.org.uk/finalReport.htm]

52. Yoon, M. J.; Bae, Y. S.; Son, S. H.; Lee, J. W.; Lee, C. H., Comparison of YAG : Eu phosphors synthesized by supercritical water in batch and continuous reactors. Korean J. Chem. Eng. 2007, $24,877-880$.

53. Atashfaraz, M.; Shariaty-Niassar, M.; Ohara, S.; Minami, K.; Umetsu, M.; Naka, T.; Adschiri, T., Effect of titanium dioxide solubility on the formation of $\mathrm{BaTiO}_{3}$ nanoparticles in supercritical water. Fluid Phase Equilib. 2007, 257, 233-237.

54. Arita, T.; Moriya, K.-I.; Minami, K.; Naka, T.; Adschiri, T., Supercritical hydrothermal synthesis of carboxylic acid-surface-functionalized $\mathrm{TiO}_{2}$ nanocrystals: $\mathrm{pH}$ sensitive dispersion and hybridization with organic compounds. Chem. Lett. 2010, 39, 961-963.

55. Galkin, A. A.; Lunin, V. V., Water in sub- and supercritical states as a universal medium for chemical reactions. Usp. Khim. 2005, 74, 24-40. 
56. Sanchez-Oneto, J.; Portela, J. R.; Nebot, E.; de la Ossa, E. M., Hydrothermal oxidation:

Application to the treatment of different cutting fluid wastes. J. Hazard. Mater. 2007, 144, 639644 .

57. Suzuki, K.; Moriyama, M.; Yamasaki, Y.; Takahashi, Y.; Inoue, C., Useful ingredients recovery from sewage sludge by using hydrothermal reaction. In AIP Conf. Proc., 2006, 75-77.

58. Aymonier, C.; Loppinet-Serani, A.; Reveron, H.; Garrabos, Y.; Cansell, F., Review of Supercrit. Fluids in inorganic materials science. J. Supercrit. Fluids 2006, 38, 242-251.

59. Marre, S.; Aymonier, C., Preparation of Nanomaterials in Flow at Supercritical Conditions from Coordination Complexes, Springer International Publishing: 2016.

6o. Sui, R.; Charpentier, P., Synthesis of metal oxide nanostructures by direct sol-gel chemistry in Supercrit. Fluids. Chem. Rev. 2012, 112, 3057-3082.

61. Fang, Z., Rapid Production of Micro- and Nano-particles Using Supercritical Water,. Springer-Verlag Berlin Heidelberg, 2010.

62. Cansell, F.; Aymonier, F., Design of functional nanostructured materials using Supercrit. Fluids. J. Supercrit. Fluids 2009, 47, 508-516.

63. Kellici, S.; Rehman, I. U.; Darr, J. A., Controlled growth of titania nanospheres in supercritical carbon dioxide using a novel surfactant stabilised precursor J. Mater. Chem. 2006, 16, 159-161.

64. Adschiri, T., Supercritical hydrothermal synthesis of organic-inorganic hybrid nanoparticles. Chem. Lett. 2007, 36, 1188-1193.

65. Veriansyah, B.; Park, H.; Kim, J. D.; Min, B. K.; Shin, Y. H.; Lee, Y. W.; Kim, J., Characterization of surface-modified ceria oxide nanoparticles synthesized continuously in supercritical methanol. J. Supercrit. Fluids 2009, 50, 283-291. 
66. Veriansyah, B.; Kim, J. D.; Min, B. K.; Shin, Y. H.; Lee, Y. W.; Kim, J., Continuous synthesis of surface-modified zinc oxide nanoparticles in supercritical methanol. J. Supercrit. Fluids 2010, 52, 76-83.

67. Veriansyah, B.; Kim, J. D.; Min, B. K.; Kim, J., Continuous synthesis of magnetite nanoparticles in supercritical methanol. Mater. Lett. 2010, 64, 2197-2200.

68. Bonggeun, S.; Naechul, S.; Young-Ho, L.; Ki Ho, A.; Youn-Woo, L., Synthesis of indium tin oxide (ITO) nanoparticles in supercritical methanol. J. Supercrit. Fluids 2016, 113, 39-43.

69. Kim, J.; Hong, S.-A.; Yoo, J., Continuous synthesis of hierarchical porous ZnO microspheres in supercritical methanol and their enhanced electrochemical performance in lithium ion batteries. Chem. Eng. J. 2015, 266, 179-188.

70. Choi, H.; Veriansyah, B.; Kim, J.; Kim, J. D.; Kang, J. W., Continuous synthesis of metal nanoparticles in supercritical methanol. J. Supercrit. Fluids 2010, 52, 285-291.

71. Adschiri, T.; Kanazawa, K.; Arai, K., Rapid and Continuous hydrothermal synthesis of Boehmite particles in subcritical and supercritical water. J. Am. Ceram. Soc. 1992, 75, 2615-2618.

72. Adschiri, T.; Kanazawa, K.; Arai, K., Rapid and continuous hydrothermal crystallization of metaloxide particles in supercritical water. J. Am. Ceram. Soc. 1992, 75, 1019-1022.

73. Adschiri, T.; Arai, K.; Onai, S.; Yamane, S., Hydrothermal synthesis of metal oxide fine particles in supercritical water. In 3rd Int. Symp. Supercrit. Fluids, Bordeaux, France, 1994, T3, 241-246.

74. Smith, R. L.; Atmaji, P.; Hakuta, Y.; Kawaguchi, M.; Adschiri, T.; Arai, K., Recovery of metals from simulated high-level liquid waste with hydrothermal crystallization. J. Supercrit. Fluids 1997, 11, $103-114$.

75. Cabanas, A.; Darr, J. A.; Lester, E.; Poliakoff, M., Continuous hydrothermal synthesis of inorganic materials in a near-critical water flow reactor; the one-step synthesis of nano-particulate $\mathrm{Ce}_{1}$ $\mathrm{ZZr}_{x} \mathrm{O}_{2}(\mathrm{x}=0-1)$ solid solutions. J. Mater. Chem. 2001, 11, 561-568. 
76. Chaudhry, A. A.; Haque, S.; Kellici, S.; Boldrin, P.; Rehman, I.; Fazal, A. K.; Darr, J. A., Instant nano-hydroxyapatite: a continuous and rapid hydrothermal synthesis. Chem. Commun. 2006, 2286-2288.

77. Hakuta, Y.; Adschiri, T.; Suzuki, T.; Chida, T.; Seino, K.; Arai, K., Flow method for rapidly producing barium hexaferrite particles in supercritical water. J. Am. Ceram. Soc. 1998, 81, 24612464 .

78. Hakuta, Y.; Hayashi, H.; Arai, K., Fine particle formation using Supercrit. Fluids. Curr. Op. Solid State Mater. Sci. 2003, 7, 341-351.

79. Hakuta, Y.; Hayashi, H.; Arai, K., Hydrothermal synthesis of photocatalyst potassium hexatitanate nanowires under supercritical conditions. J. Mater. Sci. 2004, 39, 4977-4980.

8o. Hakuta, Y.; Ohashi, T.; Hayashi, H.; Arai, K., Hydrothermal synthesis of zirconia nanocrystals in supercritical water. J. Mater. Res. 2004, 19, 2230-2234.

81. Hakuta, Y.; Ura, H.; Hayashi, H.; Arai, K., Effects of hydrothermal synthetic conditions on the particle size of gamma-AIO(OH) in sub and supercritical water using a flow reaction system. Mater. Chem. Phys. 2005, 93, 466-472.

82. Hakuta, Y.; Ura, H.; Hayashi, H.; Arai, K., Continuous production of $\mathrm{BaTiO}_{3}$ nanoparticles by hydrothermal synthesis. Ind. Eng. Chem. Res. 2005, 44, 840-846.

83. Hakuta, Y.; Ura, H.; Hayashi, H.; Arai, K., Effect of water density on polymorph of $\mathrm{BaTiO}_{3}$ nanoparticles synthesized under sub and supercritical water conditions. Mater. Lett. 2005, 59, 1387-1390.

84. Sue, K.; Adschiri, T.; Arai, K., Predictive model for equilibrium constants of aqueous inorganic species at subcritical and supercritical conditions. Ind. Eng. Chem. Res. 2002, 41, 3298-3306.

85. Sue, K.; Hakuta, Y.; Smith, R. L.; Adschiri, T.; Arai, K., Solubility of lead(II) oxide and copper(II) oxide in subcritical and supercritical water. J. Chem. Eng. Data 1999, 44, 1422-1426. 
86. Sue, K.; Kakinuma, N.; Adschiri, T.; Arai, K., Continuous production of nickel fine particles by hydrogen reduction in near-critical water. Ind. Eng. Chem. Res. 2004, 43, 2073-2078.

87. Sue, K.; Kimura, K.; Murata, K.; Arai, K., Effect of cations and anions on properties of zinc oxide particles synthesized in supercritical water. J. Supercrit. Fluids 2004, 30, 325-331.

88. Sue, K.; Suzuki, M.; Arai, K.; Ohashi, T.; Ura, H.; Matsui, K.; Hakuta, Y.; Hayashi, H.; Watanabe, M.; Hiaki, T., Size-controlled synthesis of metal oxide nanoparticles with a flow-through supercritical water method. Green Chem. 2006, 8, 634-638.

89. Sue, K. W.; Kimura, K.; Yamamoto, M.; Arai, K., Rapid hydrothermal synthesis of ZnO nanorods without organics. Mater. Lett. 2004, 58, 3350-3352.

9o. Quesada-Cabrera, R.; Powell, M. J.; Marchand, P.; Denis, C. J.; Di Maggio, F.; Darr, J. A.; Parkin, I. P., Scalable production of thermochromic $\mathrm{Nb}$-doped $\mathrm{VO}_{2}$ nanomaterials using continuous hydrothermal flow synthesis. J. Nanosci. Nanotech. 2016, 16, 10104-10111.

91. Weng, X.; Boldrin, P.; Abrahams, I.; Skinner, S. J.; Darr, J. A., Direct syntheses of mixed ion and electronic conductors $\mathrm{La}_{4} \mathrm{Ni}_{3} \mathrm{O}_{10}$ and $\mathrm{La}_{3} \mathrm{Ni}_{2} \mathrm{O}_{7}$ from nanosized coprecipitates. Chem. Mater. 2007, $19,4382-4384$

92. Weng, X.; Zhang, J.; Wu, Z.; Liu, Y.; Wang, H.; Darr, J. A., Continuous hydrothermal syntheses of highly active composite nanocatalysts. Green Chem. 2011, 13, 850-853.

93. Marchand, P.; Makwana, N. M.; Tighe, C. J.; Gruar, R. I.; Parkin, I. P.; Carmalt, C.; Darr, J. A., Highthroughput synthesis, screening and scale-Up of optimised conducting indium tin oxides. ACS Combi. Sci. 2016, 18, 130-137.

94. Cabanas, A.; Li, J.; Blood, P.; Chudoba, T.; Lojkowski, W.; Poliakoff, M.; Lester, E., Synthesis of nanoparticulate yttrium aluminum garnet in supercritical water-ethanol mixtures. The J. Supercrit. Fluids 2007, 40, 284-292. 
95. Kim, J.; Park, Y. S.; Veriansyah, B.; Kim, J. D.; Lee, Y. W., Continuous synthesis of surfacemodified metal oxide nanoparticles using supercritical methanol for highly stabilized nanofluids. Chem. Mater. 2008, 20, 6301-6303.

96. Gong, K.; Rehman, I. U.; Darr, J. A., Characterization and drug release investigation of amorphous drug-hydroxypropyl methylcellulose composites made via supercritical carbon dioxide assisted impregnation. J. Pharm. Biomed. Anal. 2008, 48, 1112-1119.

97. Gong, K.; Braden, M.; Patel, M. P.; Rehman, I. U.; Zhang, Z.; Darr, J. A., Controlled release of chlorhexidine diacetate from a porous methacrylate system: Supercritical fluid assisted foaming and impregnation. J. Pharm. Sci. 2007, 96, 2048-2056.

98. Gong, K.; Rehman, I. U.; Darr, J. A., Synthesis of poly(sebacic anhydride)-indomethacin controlled release composites via supercritical carbon dioxide assisted impregnation. Int. J. Pharm. 2007, $338,191-197$

99. Gong, K.; Darr, J. A.; Rehman, I. U., Supercritical fluid assisted impregnation of indomethacin into chitosan thermosets for controlled release applications. Int. J. Pharm. 2006, 315, 93-98.

100. Gong, K.; Viboonkiat, R.; Rehman, I. U.; Buckton, G.; Darr, J. A., Formation and characterization of porous indomethacin-PVP coprecipitates prepared using solvent-free supercritical fluid processing. J. Pharm. Sci. 2005, 94, 2583-2590.

101. Partap, S.; Muthutantri, A.; Rehman, I. U.; Davis, G. R.; Darr, J. A., Preparation and characterisation of controlled porosity alginate hydrogels made via a simultaneous micelle templating and internal gelation process. J. Mater. Sci. 2007, 42, 3502-3507.

102.Partap, S.; Rehman, I.; Jones, J. R.; Darr, J. A., "Supercritical carbon dioxide in water" emulsiontemplated synthesis of porous calcium alginate hydrogels. Adv. Mater. 2006, 18, 501-504. 
103. Partap, S.; Hebb, A. K.; Rehman, I. U.; Darr, J. A., Formation of porous natural-synthetic polymer composites using emulsion templating and supercritical fluid assisted impregnation. Polym. Bull. $2007,58,849-860$.

104.Ma, J.; Bilotti, E.; Peijs, T.; Darr, J. A., Preparation of polypropylene/sepiolite nanocomposites using supercritical $\mathrm{CO}_{2}$ assisted mixing. Eur. Polym. J. 2007, 43, 4931-4939.

105. Adschiri, T.; Lee, Y. W.; Goto, M.; Takami, S., Green materials synthesis with supercritical water. Green Chem. 2011, 13, 1380-1390.

106. Weingartner, H.; Franck, E. U., Supercritical water as a solvent. Angew. Chem.-Int. Ed. 2005, 44, $2672-2692$.

107. Fernandez, D. P.; Mulev, Y.; Goodwin, A. R. H.; Sengers, J. M. H. L., A Database for the static dielectric-constant of water and steam. J. Phys. Chem. Ref. Data 1995, 24, 33-69.

108. Kashchiev, D., On the relation between nucleation work, nucleus size, and nucleation rate. J. Chem. Phys. 1982, 76, 5098-5102.

109. Cushing, B. L.; Kolesnichenko, V. L.; O'Connor, C. J., Recent advances in the liquid-phase syntheses of inorganic nanoparticles. Chem. Rev. 2004, 104, 3893-3946.

110. Simonet, V.; Calzavara, Y.; Hazemann, J. L.; Argoud, R.; Geaymond, O.; Raoux, D., X-ray absorption spectroscopy studies of ionic association in aqueous solutions of zinc bromide from normal to critical conditions. J. Chem. Phys. 2002, 117, 2771-2781.

111. Hoffmann, M. M.; Darab, J. G.; Palmer, B. J.; Fulton, J. L., A transition in the $\mathrm{Ni}^{2+}$ complex structure from six- to four-coordinate upon formation of ion pair species in supercritical water: An X-ray absorption fine structure, near-infrared, and molecular dynamics study. J. Phys. Chem. A 1999, 103, 8471-8482. 
112. Simonet, V.; Calzavara, Y.; Hazemann, J. L.; Argoud, R.; Geaymond, O.; Raoux, D., Structure of aqueous $\mathrm{ZnBr}_{2}$ solution probed by $\mathrm{X}$-ray absorption spectroscopy in normal and hydrothermal conditions. J. Chem. Phys. 2002, 116, 2997-3006.

113. Seward, T. M.; Henderson, C. M. B.; Charnock, J. M.; Dobson, B. R., An X-ray absorption (EXAFS) spectroscopic study of aquated $\mathrm{Ag}^{+}$in hydrothermal solutions to $35^{\circ}{ }^{\circ} \mathrm{C}$. Geochim. Cosmochim. Acta 1996, 60, 2273-2282.

114. Balbuena, P. B.; Johnston, K. P.; Rossky, P. J., Molecular dynamics simulation of electrolyte solutions in ambient and supercritical water. 2: Relative acidity of $\mathrm{HCl}$. J. Phys. Chem. 1996, 100, 2716-2722.

115. Dunne, P. W.; Munn, A. S.; Starkey, C. L.; Huddle, T. A.; Lester, E. H., Continuous-flow hydrothermal synthesis for the production of inorganic nanomaterials. Phil. Trans. R. Soc. A 2015, 373, Article Number: 20150015.

116. Kashchiev, D.; van Rosmalen, G. M., Review: Nucleation in solutions revisited. Cryst. Res. Technol. 2003, 38, 555-574.

117. Byrappa, K.; Oharaa, S.; Adschiri, T., Nanoparticles synthesis using supercritical fluid technology towards biomedical applications. Adv Drug Del. Rev. 2008, 60, 299-327.

118. Adschiri, T.; Takami, S.; Minami, K.; Yamagata, T.; Miyata, K.; Monshita, T.; Ueda, M.;

Fukushima, K.; Ueno, M.; Okada, T.; Oshima, H.; Mitani, Y.; Asahina, S.; Unno, S., Super hybrid materials. Adv. Mater. Nanotech. 2012, 700, 145-149.

119. Lester, E.; Aksomaityte, G.; Li, J.; Gomez, S.; Gonzalez-Gonzalez, J.; Poliakoff, M., Controlled continuous hydrothermal synthesis of cobalt oxide $\left(\mathrm{CO}_{3} \mathrm{O}_{4}\right)$ nanoparticles. Prog. Cryst. Growth Charact. Mater. 2012, 58, 3-13. 
120.Shi, L.; Naik, A. J. T.; Goodall, J. B. M.; Tighe, C.; Gruar, R.; Binions, R.; Parkin, I.; Darr, J., Highly sensitive $\mathrm{ZnO}$ nanorod- and nanoprism-based $\mathrm{NO}_{2}$ gas sensors: size and shape control using a continuous hydrothermal pilot plant. Langmuir 2013, 29, 10603-10609.

121. Howard, D. P.; Marchand, P.; Gordon, T.; Darr, J. A., Sustainable transparent conducting oxide nanomaterials; aluminium- and gallium-co-doped zinc oxide (AGZO). J. Nanosci. Nanotech. 2016, $16,10166-10171$.

122.Dunne, P. W.; Munn, A. S.; Starkey, C. L.; Lester, E. H., The sequential continuous-flow hydrothermal synthesis of molybdenum disulphide. Chem. Commun. 2015, 51 (19), 4048-50.

123. Mi, J. L.; N. Jensen, T.; Hald, P.; Overgaard, J.; Christensen, M.; Iversen, B. B., Glucose-assisted continuous flow synthesis of $\mathrm{Bi}_{2} \mathrm{Te}_{3}$ nanoparticles in supercritical/near-critical water. J. Supercrit. Fluids 2012, 67, 84-88.

124. Aqif A Chaudhry, J. C. K., Ihtesham Rehman, Jawwad A Darr, Rapid hydrothermal flow synthesis and characterisation of carbonate- and silicate-substituted calcium phosphates. J Biomater Appl. 2013, 28, 448-461.

125. Gibson IR, B. S., Bonfield W., Chemical characterization of silicon-substituted hydroxyapatite. J Biomed Mater Res. 1999, 44, 422-428.

126. Shibuki, K.; Takesue, M.; Aida, T. M.; Watanabe, M.; Hayashi, H.; Smith, R. L., Continuous synthesis of $\mathrm{Zn}_{2} \mathrm{SiO}_{4}: \mathrm{Mn}^{2+}$ fine particles in supercritical water at temperatures of $400-500{ }^{\circ} \mathrm{C}$ and pressures of 30-35 MPa. J. Supercrit. Fluids 2010, 54, 266-271.

127. Chaudhry, A. A; Yan, H.; Gong, K.; Inam, F.; Viola, G.; Reece, M. J.; Goodall, J. B.; Rehman, I. U.; McNeil-Watson, F. K.; Corbett, J. C.; Knowles, J. C.; Darr, J.A.; High-strength nanograined and translucent hydroxyapatite monoliths via continuous hydrothermal synthesis and optimized spark plasma sintering. Acta Biomater 2011, 7, 791-799. 
128. Chaudhry, A. A.; Yan, H.; Viola, G.; Reece, M. J.; Knowles, J. C.; Gong, K.; Rehman, I.; Darr, J. A., Phase stability and rapid consolidation of hydroxyapatite-zirconia nano-coprecipitates made using continuous hydrothermal flow synthesis. J. Biomater. Appl. 2012, 27, 79-90.

129. Gimeno-Fabra, M.; Hild, F.; Dunne, P. W.; Walton, K.; Grant, D. M.; Irvine, D. J.; Lester, E. H., Continuous synthesis of dispersant-coated hydroxyapatite plates. Cryst Eng Comm 2015, 17, 61756182.

130. Yu, W.; Lin, C.; Hon, L.; Zhao, Y.; Rao, Q., Rapid and continuous synthesis of carbonated hydroxyapatite in supercritical water. Chinese J. Inorg. Chem. 2008, 24, 1539-1542.

131. Kubota, S.; Morioka, T.; Takesue, M.; Hayashi, H.; Watanabe, M.; Smith, R. L., Jr., Continuous supercritical hydrothermal synthesis of dispersible zero-valent copper nanoparticles for ink applications in printed electronics. J. Supercrit. Fluids 2014, 86, 33-40.

132. Matson, D. W.; Linehan, J. C.; Bean, R. M., Ultrafine iron-oxide powders generated using a flowthrough hydrothermal process. Mater. Lett. 1992, 14, 222-226.

133. Buehler, M. F.; Darab, J. G.; Matson, D. W.; Linehan, J. C. In Bench-scale synthesis of nanoscale material, Technology 2003 Conference, Anaheim, CA, USA, Anaheim, CA, USA, 1993.

134. Matson, D. W.; Linehan, J. C.; Darab, J. G.; Buehler, M. F., Nanophase Iron-Based Liquefaction Catalysts - Synthesis, Characterization, and Model-Compound Reactivity. Energy \& Fuels 1994, 8, 10-18.

135. Cabanas, A.; Poliakoff, M., The continuous hydrothermal synthesis of nano-particulate ferrites in near critical and supercritical water. J. Mater. Chem. 2001, 11, 1408-1416.

136. Boldrin, P.; Hebb, A. K.; Chaudhry, A. A.; Otley, L.; Thiebaut, B.; Bishop, P.; Darr, J. A., Direct synthesis of nanosized $\mathrm{NiCO}_{2} \mathrm{O}_{4}$ spinel and related compounds via continuous hydrothermal synthesis methods. Ind. Eng. Chem. Res. 2007, 46, 4830-4838. 
137. Xu, C.; Teja, A. S., Continuous hydrothermal synthesis of iron oxide and PVA-protected iron oxide nanoparticles. J. Supercrit. Fluids 2008, 44, 85-91.

138. Cooper, J. F. K.; Ionescu, A.; Langford, R. M.; Ziebeck, K. R. A.; Barnes, C. H. W.; Gruar, R.; Tighe, C.; Darr, J. A.; Thanh, N. T. K.; Ouladdiaf, B., Core/shell magnetism in NiO nanoparticles. J. Appl. Phys. 2013, 114, Article number 083906

139. Hakuta, Y.; Onai, S.; Terayama, H.; Adschiri, T.; Arai, K., Production of ultra-fine ceria particles by hydrothermal synthesis under supercritical conditions. J. Mater. Sci. Lett. 1998, 17, 1211-1213. 140.Galkin, A. A.; Kostyuk, B. G.; Lunin, V. V.; Poliakoff, M., Continuous reactions in supercritical water: A new route to $\mathrm{La}_{2} \mathrm{CuO}_{4}$ with a high surface area and enhanced oxygen mobility. Angew. Chem.-Int. Ed. 2000, 39, 2738-2740.

141. Hakuta, Y.; Seino, K.; Ura, H.; Adschiri, T.; Takizawa, H.; Arai, K., Production of phosphor (YAG:Tb) fine particles by hydrothermal synthesis in supercritical water. J. Mater. Chem. 1999, 9, 2671-2674.

142. Cote, L. J.; Teja, A. S.; Wilkinson, A. P.; Zhang, Z. J., Continuous hydrothermal synthesis and crystallization of magnetic oxide nanoparticles. J. Mater. Res. 2002, 17, 2410-2416.

143. Lester, E.; Blood, P. J.; Denyer, J. P.; Azzopardi, B. J.; Lib, J.; Poliakoff, M., Impact of reactor geometry on continuous hydrothermal synthesis mixing. Mater. Res. Innov. 2010, 14, 19-26.

144. Housley, D.; Huddle, T.; Lester, E.; Poliakoff, M., The use of dimensionless groups to analyse the mixing of streams with large density differences in sub-and supercritical water. Chem. Eng. J. $2016,287,350-358$

145. Toft, L. L.; Aarup, D. F.; Bremholm, M.; Hald, P.; Iversen, B. B., Comparison of T-piece and concentric mixing systems for continuous flow synthesis of anatase nanoparticles in supercritical isopropanol/water. J. Solid State Chem. 2009, 182, 491-495. 
146.Blood, P. J.; Denyer, J. P.; Azzopardi, B. J.; Poliakoff, M.; Lester, E., A versatile flow visualisation technique for quantifying mixing in a binary system: application to continuous supercritical water hydrothermal synthesis (SWHS). Chem. Eng. Sci. 2004, 59, 2853-2861.

147. Sierra-Pallares, J.; Huddle, T.; Alonso, E.; Mato, F. A.; García-Serna, J.; Cocero, M. J.; Lester, E., Prediction of residence time distributions in supercritical hydrothermal reactors working at low Reynolds numbers. Chem. Eng. J. 2016, 299, 373-385.

148. Ma, C. Y.; Wang, X. Z.; Tighe, C. J.; Darr, J. A., Modelling and simulation of counter-current and confined jet reactors for continuous hydrothermal flow synthesis of nanomaterials. In 8th IFAC Symp. Adv. Control Chem. Proc. Singapore, 2012, 109, 26-37.

149.Chen, M.; Ma, C. Y.; Mahmud, T.; Darr, J. A.; Wang, X. Z., Modelling and simulation of continuous hydrothermal flow synthesis process for nano-materials manufacture. J. Supercrit. Fluids 2011, 59, 131-139.

150. Ma, C. Y.; Tighe, C. J.; Gruar, R. I.; Mahmud, T.; Darr, J. A.; Wang, X. Z., Numerical modelling of hydrothermal fluid flow and heat transfer in a tubular heat exchanger under near critical conditions. J. Supercrit. Fluids 2011, 57, 236-246.

151. Sierra-Pallares, J.; Marchisio, D. L.; Alonso, E.; Teresa Parra-Santos, M.; Castro, F.; Jose Cocero, M., Quantification of mixing efficiency in turbulent supercritical water hydrothermal reactors. Chem. Eng. Sci. 2011, 66, 1576-1589.

152. Lester, E.; Blood, P.; Denyer, J.; Giddings, D.; Azzopardi, B.; Poliakoff, M., Reaction engineering: The supercritical water hydrothermal synthesis of nano-particles. J. Supercrit. Fluids 2006, 37, 209-214.

153. Leybros, A.; Piolet, R.; Ariane, M.; Muhr, H.; Bernard, F.; Demoisson, F., CFD simulation of ZnO nanoparticle precipitation in a supercritical water synthesis reactor. J. Supercrit. Fluids 2012, 70, 17-26. 
154. Demoisson, F.; Ariane, M.; Leybros, A.; Muhr, H.; Bernard, F., Design of a reactor operating in supercritical water conditions using CFD simulations. Examples of synthesized nanomaterials. J. Supercrit. Fluids 2011, 58, 371-377.

155. Sugioka, K.-i.; Ozawa, K.; Kubo, M.; Tsukada, T.; Takami, S.; Adschiri, T.; Sugimoto, K.; Takenaka, N.; Saito, Y., Relationship between size distribution of synthesized nanoparticles and flow and thermal fields in a flow-type reactor for supercritical hydrothermal synthesis. J. Supercrit. Fluids 2016, 109, 43-50.

156. Aimable, A.; Muhr, H.; Gentric, C.; Bernard, F.; Le Cras, F.; Aymes, D., Continuous hydrothermal synthesis of inorganic nanopowders in supercritical water: Towards a better control of the process. Powder Technol. 2009, 190, 99-106.

157. Chen, M.; Ma, C. Y.; Mahmud, T.; Lin, T.; Wang, X. Z., Population balance modelling and experimental validation for synthesis of $\mathrm{TiO}_{2}$ nanoparticles using continuous hydrothermal process. Meas. Cont. Gran. Mater. 2012, 508, 175-179.

158. Henrik L. Hellstern, J. B., Peter Hald, Martin Bremholm, Aref Mamakhel, I Iverse, a. B. B., Development of a dual-stage continuous flow reactor for hydrothermal synthesis of hybrid nanoparticles Ind. Eng. Chem. Res. 2015, 54, 8500-8508.

159. Aoki, N.; Sato, A.; Sasaki, H.; Litwinowicz, A.; Seong, G.; Aida, T.; Hojo, D.; Takami, S.; Adschiri, T., Kinetics study to identify reaction-controlled conditions for supercritical hydrothermal nanoparticle synthesis with flow-type reactors. The J. Supercrit. Fluids 2015, 110, 161-166. 16o. Masuda, Y.; Aizawa, T.; Suzuki, A., Flow visualization and numerical simulation of T-Junction mixing of high-temperature high-pressure water. J. Chem. Eng. Jpn. 2009, 42, 64-70.

161. Takami, S.; Sugioka, K.; Ozawa, K.; Tsukada, T.; Adschiri, T.; Sugimoto, K.; Takenaka, N.; Saito, Y., In-situ neutron tomography on mixing behavior of supercritical water and room temperature 
water in a tubular flow reactor. In Proc. 1oth World Conf. Neutron Rad., Lehmann, E. H.; Kaestner, A. P.; Mannes, D., Eds. 2015, 69, 564-569.

162. Takami, S.; Sugioka, K. i.; Tsukada, T.; Adschiri, T.; Sugimoto, K.; Takenaka, N.; Saito, Y., Neutron radiography on tubular flow reactor for hydrothermal synthesis: In situ monitoring of mixing behavior of supercritical water and room-temperature water. J. Supercrit. Fluids 2012, 63, 46-51.

163. Wakashima, Y.; Suzuki, A.; Kawasaki, S. i.; Matsui, K.; Hakuta, Y., Development of a new swirling micro mixer for continuous hydrothermal synthesis of nano-size particles. J. Chem. Eng. Jpn. 2007, 40,622-629.

164.Kawasaki, S. i.; Sue, K.; Ookawara, R.; Wakashima, Y.; Suzuki, A., Development of novel micro swirl mixer for producing fine metal oxide nanoparticles by continuous supercritical hydrothermal method. J. Oleo Sci. 2010, 59, 557-562.

165. Hong, S.-A.; Kim, S. J.; Chung, K. Y.; Chun, M.-S.; Lee, B. G.; Kim, J., Continuous synthesis of lithium iron phosphate $\left(\mathrm{LiFePO}_{4}\right)$ nanoparticles in supercritical water: Effect of mixing tee. J. Supercrit. Fluids 2013, 73, 70-79.

166.Kawasaki, S. i.; Sue, K.; Ookawara, R.; Wakashima, Y.; Suzuki, A.; Hakuta, Y.; Arai, K., Engineering study of continuous supercritical hydrothermal method using a T-shaped mixer: Experimental synthesis of $\mathrm{NiO}$ nanoparticles and CFD simulation. J. Supercrit. Fluids 2010, 54, 96 102.

167. Lester, E.; Huddle, T. Mixing reactor for precipitating nanoparticles. Worldwide patent [WO2014111703-A2]

168. Tighe, C. J.; Gruar, R. I.; Ma, C. Y.; Mahmud, T.; Wang, X. Z.; Darr, J. A., Investigation of countercurrent mixing in a continuous hydrothermal flow reactor. J. Supercrit. Fluids 2012, 62, 165-172. 
169.Ma, C. Y.; Liu, J. J.; Zhang, Y.; Wang, X. Z., Simulation for scale-up of a confined jet mixer for continuous hydrothermal flow synthesis of nanomaterials. J. Supercrit. Fluids 2015, 98, 211-221.

170. Gruar, R. I.; Tighe, C. J.; Darr, J. A., Scaling-up a confined jet reactor for the continuous hydrothermal manufacture of nanomaterials. Ind. Eng. Chem. Res. 2013, 52, 5270-5281.

171. Denis, C. J.; Tighe, C. J.; Gruar, R. I.; Makwana, N. M.; Darr, J. A., Nucleation and growth of cobalt oxide nanoparticles in a continuous hydrothermal reactor under laminar and turbulent flow. Cryst. Grow. Des. 2015, 15, 4256-4265.

172. Roig, Y.; Marre, S.; Cardinal, T.; Aymonier, C., Synthesis of exciton luminescent ZnO nanocrystals using continuous supercritical microfluidics. Angew. Chem. Int. Ed. 2011, 50, 12071-12074.

173. Jensen, K. M. O.; Tyrsted, C.; Bremholm, M.; Iversen, B. B., In Situ studies of solvothermal synthesis of energy materials Chem. Sus. Chem. 2014, 7, 1594-1611.

174. Tyrsted, C.; Lock, N.; Jensen, K. M. O.; Christensen, M.; Bøjesen, E. D.; Emerich, H.; Vaughan, G.; Billinge, S. J. L.; Iversen, B. B., Evolution of atomic structure during nanoparticle formation. IUCrJ 2014, 1, 165-171.

175. Jensen, K. M. O.; Andersen, H. L.; Tyrsted, C.; Bøjesen, E. D.; Dippel, A.-C.; Lock, N.; Billinge, S. J. L.; Iversen, B. B.; Christensen, M., Mechanisms for iron oxide formation under hydrothermal conditions: An in situ total scattering study ACS Nano, 2014, 8, 10704-10714.

176. Bøjesen, E. D.; Jensen, K. M. O.; Tyrsted, C.; Lock, N.; Christensen, M.; Iversen, B. B., In situ powder diffraction study of the hydrothermal synthesis of ZnO nanoparticles Cryst. Growth Des. 2014, 14, 2803-2810.

177. Saha, D.; Jensen, K. M. O.; Tyrsted, C.; Bøjesen, E. D.; Mamakhel, A. H.; Dippel, A.-C.; Christensen, $\mathrm{M}_{\text {; }}$ Iversen, B. B., In situ total X-ray scattering study of $\mathrm{WO}_{3}$ nanoparticle formation under hydrothermal conditions Angew. Chem. Int. Ed. 2014, 53, 3667-3670. 
178. Birgisson, S.; Jensen, K. M. Ø.; Christiansen, T. L.; Von Bülow, J. F.; Iversen, B. B., In situ powder X-ray diffraction study of the hydro-thermal formation of $\mathrm{LiMn}_{2} \mathrm{O}_{4}$ nanocrystallites. Dalton Trans. 2014, 43, 15075-15084.

179. Mi, J.-L.; Jensen, K. M. O.; Tyrsted, C.; Bremholm, M.; Iversen, B. B., In situ total X-ray scattering study of the formation mechanism and structural defects in anatase $\mathrm{TiO}_{2}$ nanoparticles under hydrothermal conditions. Cryst. Eng. Comm. 2015, 17, 6868-6877.

180.Saha, D.; Bøjesen, E. D.; Jensen, K. M. O.; Dippel, A.-C.; Iversen, B. B., Formation mechanisms of Pt and $\mathrm{Pt}_{3} \mathrm{Gd}$ nanoparticles under solvothermal conditions: an in situ total X-ray scattering study. J. Phys. Chem. C 2015, 119, 13357-13362.

181. Daniel, M. C.; Astruc, D., Gold nanoparticles: Assembly, supramolecular chemistry, quantum-sizerelated properties, and applications toward biology, catalysis, and Nanotech.. Chem. Rev. 2004, $104,293-346$.

182. Bogart, L. K.; Pourroy, G.; Murphy, C. J.; Puntes, V.; Pellegrino, T.; Rosenblum, D.; Peer, D.; Lévy, R., Nanoparticles for imaging, sensing, and therapeutic intervention. ACS Nano 2014, 8, 31073122.

183. In, J. H.; Lee, H. C.; Yoon, M. J.; Lee, K. K.; Lee, J. W.; Lee, C. H., Synthesis of nano-sized YAG : $\mathrm{Eu}^{3+}$ phosphor in continuous supercritical water system. J. Supercrit. Fluids 2007, 40, 389-396. 184. van der Weg, W. F.; Vink, A. T.; Popma, T. J. A., Concentration dependence of UV and electronexcited $\mathrm{Tb}^{3+}$ luminescence in $\mathrm{Y}_{3} \mathrm{Al}_{5} \mathrm{O}_{12}$. J.Appl.Phys. 1985, 57, 5450-5456. 185.van der Weg, W. F.; Robertson, J. M.; Zwicker, W. K.; Popma, T. J. A., Cathodoluminescence saturation effects in rare earth doped materials. J. Lumin. 1981, 24-25, 633-636. 186. Hoghooghi, B.; Healey, L.; Powell, S.; McKittrick, J.; Sluzky, E.; Hesse, K., Synthesis of YAG:Cr Phosphors by Precipitation from aluminum and yttrium sulfate solutions. Mater. Chem. Phys. $1994,38,175-180$. 
187. Rao, R. P., Preparation and characterization of fine-grain yttrium-based phosphors by sol-gel process. J. Electrochem. Soc. 1996, 143, 189-197.

188. Kinsman, K. M.; McKittrick, J., Phase development and luminescence in chromium-doped yttrium aluminum garnet (YAG:Cr) phosphors. J. Am. Ceram. Soc. 1994, 77, 2866-2872.

189.Lopez, O. A.; McKittrick, J.; Shea, L. E., Fluorescence properties of polycrystalline Tm³ $3^{+}$-activated $\mathrm{Y}_{3} \mathrm{Al}_{5} \mathrm{O}_{12}$ and $\mathrm{Tm}^{3+}-\mathrm{Li}^{+}$co-activated $\mathrm{Y}_{3} \mathrm{Al}_{5} \mathrm{O}_{12}$ in the visible and near IR ranges. J. Lumin. 1997, 71, 111.

19o.Zhou, Y. H.; Lin, J.; Yu, M.; Han, S. M.; Wang, S. B.; Zhang, H. J., Morphology control and luminescence properties of YAG : Eu phosphors prepared by spray pyrolysis. Mater. Res. Bull. 2003, 38, 1289-1299.

191. Kang, Y. C.; Chung, Y. S.; Park, S. B., Preparation of YAG : Europium red phosphors by spray pyrolysis using a filter-expansion aerosol generator. J. Am. Ceram. Soc. 1999, 82, 2056-2060. 192.Kang, Y. C.; Lenggoro, I. W.; Park, S. B.; Okuyama, K., Photoluminescence characteristics of YAG:Tb phosphor particles with spherical morphology and non-aggregation. J. Phys. Chem. Solids 1999, 60, 1855-1858.

193. Sahraneshin, A.; Takami, S.; Minami, K.; Hojo, D.; Arita, T.; Adschiri, T., Synthesis and morphology control of surface functionalized nanoscale yttrium aluminum garnet particles via supercritical hydrothermal method,. Prog. Cryst. Growth Charact. Mater. 2012, 58, 43-50.

194.Zheng, Q. X.; Li, B.; Zhang, H. D.; Zheng, J. J.; Jiang, M. H.; Tao, X. T., Fabrication of YAG monodispersed particles with a novel combination method employing supercritical water process. J. Supercrit. Fluids 2009, 50, 77-81.

195. Yoon, M. J.; Son, S. H.; Lee, J. H.; Lee, J. W.; Lee, C. H., Synthesis of YAG:Ce phosphor particles using continuous supercritical water system. Ind. Eng. Chem. Res. 2006, 47, 5994-6000 
196. Hakuta, Y.; Haganuma, T.; Sue, K.; Adschiri, T.; Arai, K., Continuous production of phosphor YAG : Tb nanoparticles by hydrothermal synthesis in supercritical water. Mater. Res. Bull. 2003, 38, $1257-1265$

197. Adschiri, T.; Hakuta, Y.; Arai, K., Hydrothermal synthesis of metal oxide fine particles at supercritical conditions. Ind. Eng. Chem. Res. 2000, 39, 4901-4907.

198. Nørby, P.; Jensen, K. M. Ø.; Lock, N.; Christensen, M.; Iversen, B. B., Continuous flow supercritical water synthesis and temperature-dependent defect structure analysis of YAG and YbAG Nanoparticles. Cryst. Grow. Des. 2016, 16, 2646-2652.

199. Chudoba, T.; Lester, E.; Lojkowski, W.; Poliakoff, M.; Li, J.; Grzanka, E.; Presz, A., Synthesis of nano-sized yttrium-aluminum garnet in a continuous-flow reactor in Supercrit. Fluids. Z. fur Nat. Section B-A J. Chem. Sci. 2008, 63, 756-764.

200. Lee, J. W.; Lee, J. H.; Woo, E. J.; Ahn, H.; Kim, J. S.; Lee, C. H., Synthesis of nanosized Ce ${ }^{3^{+}}$, Eu $^{3+}$-codoped YAG phosphor in a continuous supercritical water system. Ind. Eng. Chem. Res. 2008, 47, 5994-6000.

201.Lencka, M. M.; Riman, R. E., Thermodynamic modeling of hydrothermal synthesis of ceramic powders. Chem. Mater. 1993, 5, 61-70.

202. Hoffmann, M. M.; Conradi, M. S., J. Phys. Chem. B 1998, 102, 263-271. 203. Reveron, H.; Aymonier, C.; Loppinet-Serani, A.; Elissalde, C.; Maglione, M.; Cansell, F., Singlestep synthesis of well-crystallized and pure barium titanate nanoparticles in Supercrit. Fluids. Nanotech. 2005, 16, 1137-1143.

204. Vanheusden, K.; Seager, C. H.; Warren, W. L.; Tallant, D. R.; Caruso, J.; HampdenSmith, M. J.; Kodas, T. T., Green photoluminescence efficiency and free-carrier density in ZnO phosphor powders prepared by spray pyrolysis. J. Lumin. 1997, 75, 11-16. 
205. Vanheusden, K.; Warren, W. L.; Seager, C. H.; Tallant, D. R.; Voigt, J. A.; Gnade, B. E., Mechanisms behind green photoluminescence in ZnO phosphor powders. J. Appl. Phys. 1996, 79, 7983-7990.

206. Yang, P. D.; Yan, H. Q.; Mao, S.; Russo, R.; Johnson, J.; Saykally, R.; Morris, N.; Pham, J.; He, R. R.; Choi, H. J., Controlled growth of ZnO nanowires and their optical properties. Adv. Funct. Mater. 2002, 12, 323-331.

207. Liu, Z. S.; Jing, X. P.; Song, H. W.; Fan, L. B., The relationships between UV emission and green emission in ZnO phosphor. Acta Phys.-Chim. Sin. 2006, 22, 1383-1387.

208. Ohara, S.; Mousavand, T.; Umetsu, M.; Takami, S.; Adschiri, T.; Kuroki, Y.; Takata, M., Hydrothermal synthesis of fine zinc oxide particles under supercritical conditions. Solid State Ionics 2004, 172, 261-264.

209. Li, Y. Y.; Li, Y. X.; Wu, Y. L.; Sun, W. L., Preparation and photoluminescent properties of zinc oxide phosphor. J. Lumin. 2007, 126, 177-181.

210.Gadhe, J. B.; Gupta, R. B., Hydrogen production by methanol reforming in supercritical water: Catalysis by in-situ-generated copper nanoparticles. Int. J. Hydrogen Energy 2007, 32, 2374-2381. 211. Han, N. S.; Shim, H. S.; Seo, J. H.; Park, S. M.; Min, B. K.; Kim, J.; Song, J. K., Optical properties and lasing of ZnO nanoparticles synthesized continuously in Supercrit. Fluids. Chem. Phys. Lett. 2011, 505, 51-56.

212.Ilin, E. S.; Marre, S.; Jubera, V.; C., A., Continuous supercritical synthesis of high quality UVemitting ZnO nanocrystals for optochemical applications. J. Mater. Chem. C 2013, 1, 5058-5063. 213. Tang, S. Synthesis of nanomaterials for biomedical applications. PhD Thesis, Synthesis of nanomaterials for biomedical applications, University of Nottingham, 2014. http://eprints.nottingham.ac.uk/14101/ 
214. Hobbs, H.; Briddon, S.; Lester, E., The synthesis and fluorescent properties of nanoparticulate $\mathrm{ZrO}_{2}$ doped with Eu using continuous hydrothermal synthesis. Green Chem. 2009, 11, 484-491. 215. Toyama, S.; Takesue, M.; Aida, T. M.; Watanabe, M.; Smith, R. L., Easy emission-color-control of Mn-doped zinc silicate phosphor by use of $\mathrm{pH}$ and supercritical water conditions. J. Supercrit. Fluids 2015, 98, 65-69.

216.Sue, K.; Kawasaki, S.; Sato, T.; Nishio-Hamane, D.; Hakuta, Y.; Furuya, T., Continuous

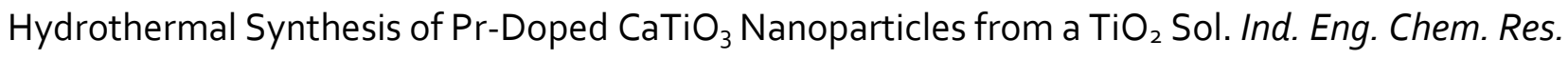
$2016,55,7628-7634$

217. Gruar, R. I.; Tighe, C. J.; Muir, J.; Kittler, J. T.; Wodjak, M.; Kenyon, A. J.; Darr, J. A., Continuous hydrothermal synthesis of surface-functionalised nanophosphors for biological imaging. RSC Adv. 2012, 2, 10037-10047.

218. Lin, T.; Kellici, S.; Gong, K.; Thompson, K.; Evans, J. R.; Wang, X.; Darr, J. A., Rapid automated materials synthesis instrument: exploring the composition and heat-treatment of nanoprecursors toward low temperature red phosphors. J. Comb. Chem. 2010, 12, 383-392.

219.Demoisson, F.; Ariane, M.; Piolet, R.; Bernard, F., Original supercritical water device for continuous production of nanopowders. Adv. Eng. Mater. 2011, 13, 487-493.

220. Machida, H.; Takesue, M.; Smith, R. L., Green chemical processes with supercrit. fluids: properties, materials, separations and energy. J. Supercrit. Fluids 2011, 60, 2-15. 221. Luckey, T. D., Nurture with ionizing radiation: A provocative hypothesis Nutrit. Cancer . Int. J. 1999, 34, 1-11.

222. Berwick, M.; Kesler, D., Ultraviolet radiation exposure, vitamin D, and cancer. Photochem. Photobiol. 2005, 81, 1261-1266.

223. Diffey, B. L.; Tanner, P. R.; Matts, P. J.; Nash, J. F., In vitro assessment of the broad-spectrum ultraviolet protection of sunscreen products. J. Am. Acad. Derm. 2000, 43, 1024-1035. 
224. Diffey, B. L., Sunscreens and UVA protection: A major issue of minor importance. Photochem. Photobiol. 2001, 74, 61-63.

225.Sutherland, B. M.; Cimino, J. S.; Delihas, N.; DelihasS, N.; Shih, A. G.; Oliver, R. P., Ultraviolet light-induced transformation of human cells to anchorage independent growth. Cancer Res. $1985,40,2409-2411$.

226. Leffell, D. J., The other side of the sun. Lancet 2000, 356, 699-699.

227. Leffell, D. J., The scientific basis of skin cancer. J. Am. Acad. Derm. 2000, 42, S18-S22.

228. Sousa, A. R.; Amorim, K. L. E.; Medeiros, E. S.; Melo, T. J. A; Rabello, M. S., The combined effect of Photodegradation and stress cracking in polystyrene. Polym. Degrad. Stab. 2006, 91, $1504-1512$.

229. Decker, C.; Biry, S.; Zahouily, K., Photostabilization of Organic Coatings. Polym. Degrad. Stab. 1995, 49, 111-119.

230. Odonnell, B.; White, J. R., Photooxidation of polystyrene under load. J. Mater. Sci. 1994, 29, 39553963.

231. Smith, C. A.; Gerlock, J. L.; Carter, R. O., Determination of ultraviolet light absorber longevity and distribution in automotive paint systems using ultraviolet micro-spectroscopy. Polym. Degrad. Stab. 2001, 72, 89-97.

232. Modirshahla, N.; Behnajady, M. A., Photooxidative degradation of Malachite Green (MG) by $\mathrm{UV} / \mathrm{H}_{2} \mathrm{O}_{2}$ : Influence of operational parameters and kinetic modeling. Dyes and Pigments 2006, 70, 54-59.

233. Muruganandham, M.; Swaminathan, M., Advanced oxidative decolourisation of Reactive Yellow 14 azo dye by UV/TiO $, U V / \mathrm{H}_{2} \mathrm{O}_{2}, \mathrm{UV} / \mathrm{H}_{2} \mathrm{O}_{2} / \mathrm{Fe}^{2+}$ processes - a comparative study. Sep. Purif. Technol. 2006, 48, 297-303. 
234. Conrad, K. R.; Davidson, V. J.; Mulholland, D. L.; Britt, I. J.; Yada, S., Influence of PET and PET/PEN blend packaging on ascorbic acid and color in juices exposed to fluorescent and UV light. J. Food Sci. 2005, 70, E19-E25.

235. Giese, J., Antioxidants: Tools for preventing lipid oxidation. Food Tech. 1996, 50, 73-77.

236. Gimenez, B.; Roncales, P.; Beltran, J. A., The effects of natural antioxidants and lighting conditions on the quality of salmon (Salmo salar) fillets packaged in modified atmosphere. J. Sci. Food Agric. 2005, 85, 1033-1040.

237. Urbach, F., The historical aspects of sunscreens. J. Photochem. Photobiol. B-Biol. 2001, 64, 99-104.

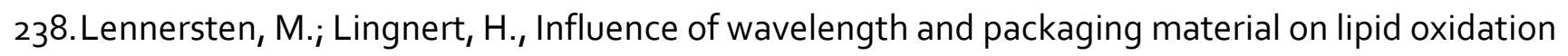
and colour changes in low-fat mayonnaise. Leben.-Wiss.t Und-Tech.-Food Sci. Tech. 2000, 33, 253260.

239. Djenane, D.; Sanchez-Escalante, A.; Beltran, J. A.; Roncales, P., Extension of the retail display life of fresh beef packaged in modified atmosphere by varying lighting conditions. J. Food Sci. 2001, 66, 181-186.

240. Djenane, D.; Sanchez-Escalante, A.; Beltran, J. A.; Roncales, P., Extension of the shelf life of beef steaks packaged in a modified atmosphere by treatment with rosemary and displayed under UV-free lighting. Meat Sci. 2003, 64, 417-426.

241. Rabello, M. S.; White, J. R., The role of physical structure and morphology in the photodegradation behaviour of polypropylene. Polym. Degrad. Stab. 1997, 56, 55-73.

242. Fechine, G. J. M.; Rabello, M. S.; Souto-Maior, R. M., The effect of ultraviolet stabilizers on the photodegradation of poly(ethylene terephthalate). Polym. Degrad. Stab. 2002, 75, 153-159.

243.Serpone, N.; Salinaro, A. E.; Horikoshi, S.; Hidaka, H., Beneficial effects of photo-inactive titanium dioxide specimens on plasmid DNA, human cells and yeast cells exposed to UVA/UVB simulated sunlight. J. Photochem. Photobiol. A-Chem. 2006, 179, 200-212. 
244http://ec.europa.eu/health/scientific committees/consumer safety en.

245.Giancaterina, S.; Ben Amor, S.; Baud, G.; Gardette, J. L.; Jacquet, M.; Perrin, C.; Rivaton, A.,

Photoprotective ceramic coatings on poly(ether ether ketone). Polymer 2002, 43, 6397-6405.

246. Guedri, B.; Ben Amor, S.; Gardette, J. L.; Jacquet, M.; Rivaton, A., Lifetime improvement of poly(ethylene naphthalate) by ZnO adhesive coatings. Polym. Degrad. Stab. 2005, 88, 199-205.

247. Han, K. Q.; YU, M. H., Study of the preparation and properties of UV-blocking fabrics of a PET/TiO 2 nanocomposite prepared by in situ polycondensation. J. Appl. Polym. Sci. 2006, 100, 1588-1593.

248. Katangur, P.; Warner, S. B.; Patra, P. K.; Kim, Y. K.; Mhetre, S. K.; Dhanote, A., Nanostructured polymer coatings for ultraviolet protection. In MRS Proc., 2003, 788, 589-594.

249. KyprianidouLeodidou, T.; Margraf, P.; Caseri, W.; Suter, U. W.; Walther, P., Polymer sheets with a thin nanocomposite layer acting as a UV filter. Polym. Adv. Technol. 1997, 8, 505-512.

250.Li, Y. Q.; Fu, S. Y.; Mai, Y. W., Preparation and characterization of transparent ZnO/epoxy nanocomposites with high-UV shielding efficiency. Polymer 2006, 47, 2127-2132.

251. Moustaghfir, A.; Rivaton, A.; Tomasella, E.; Mailhot, B.; Cellier, J.; Jacquet, M.; Gardette, J. L., Photostabilization of polycarbonate by ZnO coatings. J. Appl. Polym. Sci. 2005, 95, 380-385.

252. Muasher, M.; Sain, M., The efficacy of photo stabilizers on the color change of wood filled plastic composites. Polym. Degrad. Stab. 2006, 91, 1156-1165.

253. Nussbaumer, R. J.; Caseri, W. R.; Smith, P.; Tervoort, T., Polymer-TiO 2 nanocomposites: A route towards visually transparent broadband UV filters and high refractive index materials. Macromol. Mater. Eng. 2003, 288, 44-49.

254.Pospisil, J.; Nespurek, S., Photostabilization of coatings. Mechanisms and performance. Prog. Polym. Sci. 2000, 25, 1261-1335. 
255. Tang, E.; Cheng, G.; Pang, X.; Ma, X.; Xing, F., Synthesis of nano-ZnO/poly(methyl methacrylate) composite microsphere through emulsion polymerization and its UV-shielding property. Colloid. Polym. Sci. 2006, 284, 422-428.

256. Xiong, M. N.; Gu, G. X.; You, B.; Wu, L. M., Preparation and characterization of poly(styrene butylacrylate) latex/nano-ZnO nanocomposites. J. Appl. Polym. Sci. 2003, 90, 1923-1931.

257. Yang, M.; Pang, G. S.; Jiang, L. F.; Feng, S. H., Hydrothermal synthesis of one-dimensional zinc oxides with different precursors. Nanotech. 2006, 17, 206-212.

258. Judin, V. P. S., The Lighter Side of $\mathrm{TiO}_{2}$. Chem. Br. 1993, 29, 503-505.

259. Murphy, G. M., Sunblocks: Mechanisms of action. Photoderm. Photoim. Photomed. 1999, 15, $34-36$.

26o. Zhang, Z.; Brown, S.; Goodall, J. B.; Weng, X.; Thompson, K.; Gong, K.; Kellici, S.; Clark, R. J.; Evans, J. R.; Darr, J. A., Direct continuous hydrothermal synthesis of high surface area nanosized titania. J. Alloys Compd. 2009, 476, 451-456.

261.Tighe, C. J.; Quesada Cabrera, R.; Gruar, R. I.; Darr, J. A., Scale up production of nanoparticles: continuous supercritical water synthesis of Ce-Zn Oxides. Ind. Eng. Chem. Res. 2013, 52, 55225528.

262. Thompson, K.; Goodall, J.; Kellici, S.; Mattinson, J. A.; Egerton, T. A.; Rehman, I.; Darr, J. A., Screening tests for the evaluation of nanoparticle titania photocatalysts. J. Chem. Technol. Biotechnol. 2009, 84, 1717-1725.

263. Sue, K.; Kimura, K.; Arai, K., Hydrothermal synthesis of ZnO nanocrystals using microreactor. Mater. Lett. 2004, 58, 3229-3231.

264. Kafizas, A.; Kellici, S.; Darr, J. A.; Parkin, I. P., Titanium dioxide and composite metal/metal oxide titania thin films on glass: A comparative study of photocatalytic activity. J. Photochem. Photobiol. A-Chem.2009, 204, 183-190. 
265. Sue, K.; Murata, K.; Kimura, K.; Arai, K., Continuous synthesis of zinc oxide nanoparticles in supercritical water. Green Chem. 2003, 5, 659-662.

266. Garcia, S. P.; Steve Semancik, S. Controlling the morphology of zinc oxide nanorods crystallized from aqueous solutions: The effect of crystal growth modifiers on aspect ratio, Chem. Mater. 2007, 19, 4016-4022.

267. Goodall, J. B.; Illsley, D.; Lines, R.; Makwana, N. M.; Darr, J. A., Structure-property-composition relationships in doped zinc oxides: enhanced photocatalytic activity with rare earth dopants. ACS Combi. Sci. 2015, 17, 100-12.

268. Sondergaard, M.; Bojesen, E. D.; Christensen, M.; Iversen, B. B., Size and morphology dependence of $\mathrm{ZnO}$ nanoparticles synthesized by a fast continuous flow hydrothermal method. Cryst. Grow. Des. 2011, 11, 4027-4033.

269. Adschiri, T.; Hakuta, Y.; Sue, K.; Arai, K., Hydrothermal synthesis of metal oxide nanoparticles at supercritical conditions. J. Nanopart. Res. 2001, 3, 227-235.

270 Suchanek, W. L. Systematic study of hydrothermal crystallization of zinc oxide ( $\mathrm{ZnO}$ ) nano-sized powders with superior UV attenuation. J. Cryst. Growth. 2009, 312, 100-108.

271. Continuous Hydrothermal Synthesis of Nanomaterials: From Laboratory to Pilot Plant" project funded by EPSRC 2008 (EPSRC ref EP/E040551/1): summary available at http://gow.epsrc.ac.uk/ViewGrant.aspx?GrantRef=EP/E040551/1. .

272. Weng, X.; Zeng, Q.; Zhang, Y. G.; Dong, F.; Wu, Z., Facile approach for the syntheses of ultrafine $\mathrm{TiO}_{2}$ nanocrystallites with defects and $\mathrm{C}$ heterojunction for photocatalytic water splitting. ACS Sus. Chem. Eng. 2016, 4, 4314-4320.

273. Wakefield, G.; Lipscomb, S.; Holland, E.; Knowland, J., The effects of manganese doping on UVA absorption and free radical generation of micronised titanium dioxide and its consequences for 
the photostability of UVA absorbing organic sunscreen components. Photochem. Photobiol. Sci. $2004,3,648-652$.

274. Carp, O.; Huisman, C. L.; Reller, A., Photoinduced reactivity of titanium dioxide. Prog. Solid State Chem. 2004, 32, 33-177.

275. Tan, M. H.; Commens, C. A.; Burnett, L.; Snitch, P. J., A pilot study on the percutaneous absorption of microfine titanium dioxide from sunscreens. Aust. J. Derm. 1996, 37, 185-187. 276. Dransfield, G.; Guest, P. J.; Lyth, P. L.; McGarvey, D. J.; Truscott, T. G., Photoactivity tests of $\mathrm{TiO}_{2}$-based inorganic sunscreens - Part 1: Non-aqueous dispersions. J. Photochem. Photobiol. BBiol. 2000, 59, 147-151.

277. Dransfield, G. P., Inorganic sunscreens. Radiat. Prot. Dosim. 2000, 91, 271-273.

278. Weng, X.; Zhang, J.; Wu, Z.; Liu, Y., Continuous hydrothermal flow syntheses of transition metal oxide doped $\mathrm{Ce}_{x} \mathrm{TiO}_{2}$ nanopowders for catalytic oxidation of toluene. Catal. Today 2011, 175, 386392.

279. Goodall, J. B. M.; Kellici, S.; Illsley, D.; Lines, R.; Knowles, J. C.; Darr, J. A., Optical and photocatalytic behaviours of nanoparticles in the Ti-Zn-O binary system. RSC Adv. 2014, 4, 31799-31809.

280. Macdonald; , E. K.; Shaver, M. P., Intrinsic high refractive index polymers. Polym. Int. 2014, 64, 6-14.

281.Liu, C.; Hajagos, T. J.; Chen, D.; Chen, Y.; Kishpaugh, D.; Pei, Q., Efficient one-pot synthesis of colloidal zirconium oxide nanoparticles for high-refractive-index nanocomposites. ACS Appl. Mater. Interfaces 2016, 8, 4795-4802.

282. http://www.pixelligent.com/. 
283. Lübke, M.; Johnson, I.; Makwana, N. M.; Brett, D.; Shearing, P.; Liu, Z.; Darr, J. A., High power $\mathrm{TiO}_{2}$ and high capacity $\mathrm{Sn}$-doped $\mathrm{TiO}_{2}$ nanomaterial anodes for lithium-ion batteries. J. Power Sources 2015, 294, 94-102.

284. Kawasaki, S. I.; Xiuyi, Y.; Sue, K.; Hakuta, Y.; Suzuki, A.; Arai, K., Continuous supercritical hydrothermal synthesis of controlled size and highly crystalline anatase $\mathrm{TiO}_{2}$ nanoparticles. J. Supercrit. Fluids 2009, 50, 276-282.

285. Kawai-Nakamura, A.; Sato, T.; Sue, K.; Tanaka, S.; Saitoh, K.; Aida, K.; Hiaki, T., Rapid and continuous hydrothermal synthesis of metal and metal oxide nanoparticles with a microtubereactor at $523 \mathrm{~K}$ and $30 \mathrm{MPa}$. Mater. Lett. 2008, 62, 3471-3473.

286. Pighini, C.; Aymes, D.; Millot, N.; Saviot, L., Low-frequency Raman characterization of sizecontrolled anatase $\mathrm{TiO}_{2}$ nanopowders prepared by continuous hydrothermal syntheses. J. Nanopart. Res. 2007, 9, 309-315.

287. Malinger, K. A.; Maguer, A.; Thorel, A.; Gaunand, A.; Hochepied, J. F., Crystallization of anatase nanoparticles from amorphous precipitate by a continuous hydrothermal process. Chem. Eng. J. 2011, 174, 445-451.

288. Hayashi, H.; Ueda, A.; Suino, A.; Hiro, Y.; Hakuta, Y., Hydrothermal synthesis of yttria stabilized $\mathrm{ZrO}_{2}$ nanoparticles in subcritical and supercritical water using a flow reaction system. J. Solid State Chem. 2009, 182, 2985-2990.

289. Becker, J.; Hald, P.; Bremholm, M.; Pedersen, J. S.; Chevallier, J.; Iversen, S. B.; Iversen, B. B., Critical size of crystalline $\mathrm{ZrO}_{2}$ nanoparticles synthesized in near- and supercritical water and supercritical isopropyl alcohol. Acs Nano 2008, 2, 1058-1068.

290. Taguchi, M.; Takami, S.; Adschiri, T.; Nakane, T.; Sato, K.; Naka, T., Synthesis of surfacemodified monoclinic $\mathrm{ZrO}_{2}$ nanoparticles using supercritical water. Cryst. Eng. Comm, 2012, 14, $2132-2138$. 
291. Kaseda, K.; Takesue, M.; Aida, T. M.; Watanabe, M.; Hayashi, H.; Smith, R. L., Restructuring mechanism of $\mathrm{NbO}_{6}$ octahedrons in the crystallization of $\mathrm{KNbO}_{3}$ in supercritical water. $J$. Supercrit. Fluids 2011, 58, 279-285.

292. Buxbaum, G., Industrial inorganic pigment. Weinheim, VCH: 1993.

293. Jansen, M.; Letschert, H. P., Inorganic yellow-red pigments without toxic metals. Nature 2000, $404,980-982$.

294. Kim, S. W.; Hasegawa, T.; Watanabe, M.; Sugimoto, K.; Saito, Y.; Uematsu, K.; Toda, K.; Sato, M., Environmentally friendly $\mathrm{Rb}_{3} \mathrm{~V}_{5} \mathrm{O}_{14}$ fluorescent red pigment. Dyes and Pigments 2017, 136, 219-223.

295. Zhao, G. S.; Liu, W.; Dong, M.; Li, W. X.; Chang, L. M., Synthesis of monoclinic sheet-like $\mathrm{BiVO}_{4}$ with preferentially exposed (040) facets as a new yellow-green pigment. Dyes and Pigments 2016, 134, 91-98.

296. Kursawe, M.; Anselmann, R.; Hilarius, V.; Pfaff, G., Nanoparticles by wet chemical processing in commercial applications. J. Sol-Gel Sci. Technol. 2005, 33, 71-74.

297. Millot, N.; Xin, B.; Pighini, C.; Aymes, D., Hydrothermal synthesis of nanostructured inorganic powders by a continuous process under supercritical conditions. J. Eur. Ceram. Soc. 2005, 25, $2013-2016$.

298. Rangappa, D.; Ohara, S.; Naka, T.; Kondo, A.; Ishii, M.; Adschiri, T., Synthesis and organic modification of $\mathrm{CoAl}_{2} \mathrm{O}_{4}$ nanocrystals under supercritical water conditions. J. Mater. Chem. 2007, $17,4426-4429$.

299. group website of Prof Adschiri http://www.wpi-aimr.tohoku.ac.jp/ajiri labo/english/. 300. Service, R. F., Nanotechhology - Color-changing nanoparticles offer a golden ruler for molecules. Science 2005, 308, 1099-1099. 
301. Gil, C.; Villegas, M. A., Ruby coloured lead glasses by generation of silver nanoparticles. Mater. Chem. Phys. 2004, 88, 185-191.

302. Aksomaityte, G.; Poliakoff, M.; Lester, E., The production and formulation of silver nanoparticles using continuous hydrothermal synthesis. Chem. Eng. Sci. 2013, 85, 2-10.

303. Uppal, M. A.; Kafizas, A.; Ewing, M. B.; Parkin, I. P., The room temperature formation of gold nanoparticles from the reaction of cyclohexanone and auric acid; a transition from dendritic particles to compact shapes and nanoplates. J. Mater. Chem. A 2013, 1, 7351-7359.

304. Goodall, J. PhD Thesis, UCL London, 2009.

305. Aimable, A.; Xin, B.; Millot, N.; Aymes, D., Continuous hydrothermal synthesis of nanometric $\mathrm{BaZrO}_{3}$ in supercritical water. J. Solid State Chem. 2008, 181, 183-189.

306.Reveron, H.; Elissalde, C.; Aymonier, C.; Bidault, O.; Maglione, M.; Cansell, F., Supercritical fluid route for synthesizing crystalline barium strontium titanate nanoparticles. J. Nanosci. Nanotech.

$2005,5,1741-1744$.

307. Ricceri, R.; Ardizzone, S.; Baldi, G.; Matteazzi, P., Ceramic pigments obtained by sol-gel techniques and by mechanochemical insertion of color centers in $\mathrm{Al}_{2} \mathrm{O}_{3}$ host matrix. J. Eur. Ceram. Soc. 2002, 22, 629-637.

308.Sue, K.; Sato, T.; Kawasaki, S. i.; Takebayashi, Y.; Yoda, S.; Furuya, T.; Hiaki, T., Continuous hydrothermal synthesis of $\mathrm{Fe}_{2} \mathrm{O}_{3}$ nanoparticles using a central collision-type micromixer for rapid and homogeneous nucleation at $673 \mathrm{~K}$ and $30 \mathrm{MPa}$. Ind. Eng. Chem. Res. 2010, 49, 8841-8846. 309.Liang, M. T.; Wang, S. H.; Chang, Y. L.; Hsiang, H. I.; Huang, H. J.; Tsai, M. H.; Juan, W. C.; Lu, S. F., Iron oxide synthesis using a continuous hydrothermal and solvothermal system. Ceram. Int. 2010, 36, 1131-1135.

310. Kiri, P.; Hyett, G.; Binions, R., Solid state thermochromic materials. Adv. Mat. Lett. 2010, 1, 86105 
311. Powell, M. J.; Marchand, P.; Denis, C. J.; Bear, J. C.; Darr, J. A.; Parkin, I. P., Direct and continuous synthesis of $\mathrm{VO}_{2}$ nanoparticles. Nanoscale 2015, 7, 18686-18693.

312. Thanh, N. T. K.; Green, L. A. W., Functionalisation of nanoparticles for biomedical applications. Nano Today 2010, 5, 213-230.

313. Noimark, S.; Page, K.; Bear, J. C.; Sotelo-Vazquez, C.; Quesada-Cabrera, R.; Lu, Y.; Allan, E.; Darr, J. A.; Parkin, I. P., Functionalised gold and titania nanoparticles and surfaces for use as antimicrobial coatings. Faraday Discuss. 2014, 175, $273-87$.

314. Bioceramics: Properties, Preparations and Applications Nova publisher: 2009.

315. Wilczewska, A. Z.; Niemirowicz, K.; Markiewicz, K. H.; Car, H., Nanoparticles as drug delivery systems. Pharmacological Reports 2012, 64, 1020-1037.

316. Akira Ito; Masashige Shinkai; Hiroyuki Honda; Takeshi Kobayashi, Medical application of functionalized magnetic nanoparticles. J. Biosci. Bioeng. 2005, 100, 1-11.

317.http://www.fda.gov/ScienceResearch/SpecialTopics/Nanotech./ucm301114.htm.

318. ISO Standard 13779-1:2008 Implants for surgery. Hydroxyapatite Part 1: Ceramic hydroxyapatite.http://www.iso.org/iso/catalogue_detail.htm?csnumber=43826

319. http://www.endomagnetics.com/sentimag/.

320. World Cancer Report 2014. WHO: 2014.

321. Bañobre-López, M.; Teijeiro, A.; Rivas, J., Magnetic nanoparticle-based hyperthermia for cancer treatment. Rep. Prac. Oncol. Radio. 2013, 18, 397-400.

322.Luo, S.; Wang, L. F.; Ding, W. J.; Wang, H.; Zhou, J. M.; Jin, H. K.; Su, S. F.; W., O. W., Clinical trials of magnetic induction hyperthermia for treatment of tumours OA Cancer 2014, 2, 2. http://www.oapublishinglondon.com/article/1145 
323. Maurizi, L.; Bouyer, F.; Paris, J.; Demoisson, F.; Saviot, L.; Millot, N., One step continuous hydrothermal synthesis of very fine stabilized superparamagnetic nanoparticles of magnetite.

Chem. Commun. 2011, 47, 11706-11708.

324. Thomas, G.; Demoisson, F.; Chassagnon, R.; Popova, E.; Millot, N., One-step continuous synthesis of functionalized magnetite nanoflowers. Nanotech. 2016, 27, 135604.

325. Sue, K.; Hattori, H.; Sato, T.; Komoriya, T.; Kawai-Nakamura, A.; Tanaka, S.; Hiaki, T.; Kawasaki, S.; Takebayashi, Y.; Yoda, S.; Furuya, T., Super-rapid hydrothermal synthesis of highly crystalline and water-soluble magnetite nanoparticles using a microreactor. Chem. Lett. 2009, 38, 792-793.

326. Bremholm, M.; Felicissimo, M.; Iversen, B. B., Time-resolved in situ synchrotron X-ray study and large-scale production of magnetite nanoparticles in supercritical water. Angew. Chem.-Int. Ed. 2009, 48, 4788-4791.

327. Togashi, T.; Takami, S.; Kawakami, K.; Yamamoto, H.; Naka, T.; Sato, K.; Abe, K.; Adschiri, T., Continuous hydrothermal synthesis of 3,4-dihydroxyhydrocinnamic acid-modified magnetite nanoparticles with stealth-functionality against immunological response. J. Mater. Chem. 2012, $22(18), 9041-9045$.

328. Rashid, A. U.; Southern, P.; Darr, J. A.; Awan, S.; Manzoor, S., Strontium hexaferrite $\left(\mathrm{SrFe}_{12} \mathrm{O}_{19}\right)$ based composites for hyperthermia applications. J. Magn. Magn. Mater. 2013, 344, 134-139.

329. Eltzholtz, J. R.; Iversen, B. B., High-temperature and high-pressure pulsed synthesis apparatus for supercritical production of nanoparticles. Rev. Sci. Instrum. 2011, 82, Article 084102.

330. Sasaki, T.; Ohara, S.; Naka, T.; Vejpravova, J.; Sechovsky, V.; Umetsu, M.; Takami, S.; Jeyadevan, B.; Adschiri, T., Continuous synthesis of fine $\mathrm{MgFe}_{2} \mathrm{O}_{4}$ nanoparticles by supercritical hydrothermal reaction. J. Supercrit. Fluids 2010, 53, 92-94.

331. https://www.nice.org.uk/guidance/qs61/chapter/introduction. 
332. Morioka, T.; Takesue, M.; Hayashi, H.; Watanabe, M.; Smith, R. L., Jr., Antioxidation Properties and Surface Interactions of polyvinylpyrrolidone-capped zerovalent copper nanoparticles synthesized in supercritical water. ACS Appl Mater Interfaces 2016, 8, 1627-34.

333. Zhou, L.; Wang, S.; Ma, H.; Ma, S.; Xu, D.; Guo, Y., Size-controlled synthesis of copper nanoparticles in supercritical water. Chem. Eng. Res. Des. 2015, 98, 36-43.

334. Bersani, M.; Gupta, K.; Mishra, A. K.; Lanza, R.; Taylor, S. F. R.; Islam, H. U.; Hollingsworth, N.; Hardacre, C.; de Leeuw, N. H.; Darr, J. A., Combined EXAFS, XRD, DRIFTS, and DFT study of nano copper based catalysts for $\mathrm{CO}_{2}$ hydrogenation. ACS Catalysis 2016, 6, 5823-5833.

335. Gupta, K.; Bersani, M.; Darr, J. A., Highly efficient electro-reduction of $\mathrm{CO}_{2}$ to formic acid by nano-copper. J. Mater. Chem. A 2016, 4, 13786-13794.

336. Kellici, S.; Acord, J.; Ball, J.; Reehal, H. S.; Morgan, D.; Sah, B., A single rapid route for the synthesis of reduced graphene oxide with antibacterial activities. RSC Adv. 2014, 4, 14858 14861.

337. Kellici, S.; Acord, J.; Vaughn, A.; Power, N.; Morgan, D.; Heil, T.; Facq, S.; Lampronti, G., Calixarene assisted rapid synthesis of silver-graphene nanocomposites with enhanced antibacterial activity. ACS Appl. Mater. Int. 2016, 8 (29), 19038-19046.

338. Moshaverinia, A.; Roohpour, N.; Darr, J. A.; Rehman, I. U., Synthesis and characterization of a novel N-vinylcaprolactam-containing acrylic acid terpolymer for applications in glass-ionomer dental cements. Acta Biomat. 2009, 5, 2101-2108.

339. Moshaverinia, A.; Roohpour, N.; Darr, J. A.; Rehman, I. U., Synthesis of a proline-modified acrylic acid copolymer in supercritical $\mathrm{CO}_{2}$ for glass-ionomer dental cement applications. Acta Biomat. 2009, 5, 1656-1662.

340. Moshaverinia, A.; Ansari, S.; Movasaghi, Z.; Billington, R. W.; Darr, J. A.; Rehman, I. U., Modification of conventional glass-ionomer cements with $\mathrm{N}$-vinylpyrrolidone containing 
polyacids, nano-hydroxy and fluoroapatite to improve mechanical properties. Dent. Mater. 2008, $24,1381-1390$.

341. Moshaverinia, A.; Roohpour, N.; Billington, R. W.; Darr, J. A.; Rehman, I. U., Synthesis of Nvinylpyrrolidone modified acrylic acid copolymer in supercrit. fluids and its application in dental glass-ionomer cements. J. Mater. Sci.-Mater. Med. 2008, 19, 2705-2711.

342. Moshaverinia, A.; Ansari, S.; Moshaverinia, M.; Roohpour, N.; Darr, J. A.; Rehman, I., Effects of incorporation of hydroxyapatite and fluoroapatite nanobioceramics into conventional glass ionomer cements (GIC). Acta Biomat. 2008, 4, 432-440.

343. Moshaverinia, A.; Roohpour, N.; Ansari, S.; Moshaverinia, M.; Schricker, S.; Darr, J. A.; Rehman, I. U., Effects of N-vinylpyrrolidone (NVP) containing polyelectrolytes on surface properties of conventional glass-ionomer cements (GIC). Dent. Mater. 2009, 25, 1240-1247.

344. Biomaterials: An Introduction. Springer Science \& Business Media: 2007.

345. Hench, L. L., Bioceramics: From Concept to Clinic. Jounral of American Ceramics Society 1991, 74, 1487-1510.

346.Norton, J.; Malik, K. R.; Darr, J. A.; Rehman, I., Recent developments in processing and surface modification of hydroxyapatite. Adv. Appl. Ceram. 2006, 105, 113-139.

\section{7.http://www.ciitlahore.edu.pk/IRCBM/index.aspx.}

348. Anwar, A.; Darr, J. A.; Pankhurst, Q., Synthesis and surface modification of hydroxyapatite nanorods incorporating functionalized surfaces for dental restoration. J. Bioeng. Biomed. Sci., 2015, $5(1), 79$.

349. Anwar, A., PhD Thesis, University College London. 2015. http://discovery.ucl.ac.uk/1458257/1/Aneela's\%20PhD\%20Thesis.pdf 
350. Anwar, A.; Rehman, I. U.; Darr, J. A., Low-temperature synthesis and surface modification of high surface area calcium hydroxyapatite nanorods incorporating organofunctionalized surfaces. J. Phys. Chem. C 2016, 120, 29069-29076.

351. Khan, A. S.; Ahmed, Z.; Edirisinghe, M. J.; Wong, F. S. L.; Rehman, I. U., Preparation and characterization of a novel bioactive restorative composite based on covalently coupled polyurethane-nanohydroxyapatite fibres. Acta Biomat. 2008, 4, 1275-1287.

352. Darr, J. A.; Guo, Z. X.; Raman, V.; Bououdina, M.; Rehman, I. U., Metal organic chemical vapour deposition (MOCVD) of bone mineral like carbonated hydroxyapatite coatings. Chem. Commun. 2004, 696-697.

353. Beganskienè, A.; Dudko, O.; Sirutkaitis, R.; Giraitis, R., Water based sol-gel synthesis of hydroxyapatite. Mater. Sci. 2003, 9, 383-386.

354. Gonzalez-McQuire, R.; Chane-Ching, J.-Y.; Vignaud, E.; Lebugle, A.; Mann, S., Synthesis and characterization of amino acid-functionalized hydroxyapatite nanorods. J. Mater. Chem. 2004, 14, 2277-2281.

355. Larry Hench, http://www.boing-boingfoundation.com/books.html.

356. Chaudhry, A. A.; Goodall, J.; Vickers, M.; Cockcroft, J. K.; Rehman, I.; Knowles, J. C.; Darr, J. A., Synthesis and characterisation of magnesium substituted calcium phosphate bioceramic nanoparticles made via continuous hydrothermal flow synthesis. J. Mater. Chem. 2008, 18, 59005908.

357. Weng, X.; Brett, D.; Yufit, V.; Shearing, P.; Brandon, N.; Reece, M.; Yan, H.; Tighe, C.; Darr, J. A., Highly conductive low nickel content nano-composite dense cermets from nano-powders made via a continuous hydrothermal synthesis route. Solid State lonics 2010, 181 (17-18), 827-834. 
358. Lester, E.; Tang, S. V. Y.; Khlobystov, A.; Rose, V. L.; Buttery, L.; Roberts, C. J., Producing nanotubes of biocompatible hydroxyapatite by continuous hydrothermal synthesis. Cryst Eng Comm 2013, 15 (17), 3256-326o.

359. Piccirillo, C.; Denis, C. J.; Pullar, R. C.; Binions, R.; Parkin, I. P.; Darr, J. A.; Castro, P. M. L., Aerosol assisted chemical vapour deposition of hydroxyapatite-embedded titanium dioxide composite thin films. J. Photochem. Photobiol. A: Chem. 2017 332, 45-53.

36o.http://www.syalons.com/advanced-ceramic-materials/zirconia-ceramics/.

361. Taguchi, M.; Nakane, T.; Matsushita, A.; Sakka, Y.; Uchikoshi, T.; Funazukuri, T.; Naka, T., Onepot synthesis of monoclinic $\mathrm{ZrO}_{2}$ nanocrystals under subcritical hydrothermal conditions. J. Supercrit. Fluids 2014, 85, 57-61.

362.Saada, R.; Kellici, S.; Heil, T.; Morgan, D.; Saha, B., Greener synthesis of dimethyl carbonate using a novel ceria-zirconia oxide/graphene nanocomposite catalyst. Appl. Cat. B: Envir.2015, 168, 353362.

363. Weng, X. L.; Perston, B.; Wang, X. Z.; Abrahams, I.; Lin, T.; Yang, S. F.; Evans, J. R. G.; Morgan, D. J.; Carley, A. F.; Bowker, M.; Knowles, J. C.; Rehman, I.; Darr, J. A., Synthesis and characterization of doped nano-sized ceria-zirconia solid solutions. Appl. Cat. B: Envir. 2009, 90 (3-4), 405-415. 364. Kim, J. R.; Myeong, W. J.; Ihm, S. K., Characteristics in oxygen storage capacity of ceria-zirconia mixed oxides prepared by continuous hydrothermal synthesis in supercritical water. Appl. Cat. B: Envir. 2007, $71(1-2), 57-63$.

365. Bremholm, M.; Becker-Christensen, J.; Iversen, B., High-Pressure, High-temperature formation of phase-pure monoclinic zirconia nanocrystals studied by time-resolved in situ synchrotron X-ray diffraction. Adv. Mater. 2009, 21 (35), 3572-3575.

366.Demoisson, F.; Ariane, M.; Saviot, L., Acoustic vibrations of monoclinic zirconia nanocrystals. J. Phys. Chem. C 2011, 115 (30), 14571-14575. 
367. Kim, J. R.; Lee, K. Y.; Suh, M. J.; Ihm, S. K., Ceria-zirconia mixed oxide prepared by continuous hydrothermal synthesis in supercritical water as catalyst support. Catal. Today 2012, 185 (1), 2534.

368.Gonzalo-Juan, I.; Ferrari, B.; Colomer, M.; Rodriguez, M.; Sanchez-Herencia, A.; Koh, P. Y.; Teja, A., Synthesis and dispersion of yttria-stabilized zirconia (YSZ) nanoparticles in supercritical water. Mater. Chem. Phys. 2012, 134 (1), 451-458.

369.Galkin, A. A.; Kostyuk, B. G.; Kuznetsova, N. N.; Turakulova, A. O.; Lunin, V. V.; Polyakov, M., Unusual approaches to the preparation of heterogeneous catalysts and supports using water in subcritical and supercritical states. Kinet. Catal. 2001, 42 (2), 154-162.

370. Dunne, P. W.; Starkey, C. L.; Gimeno-Fabra, M.; Lester, E. H., The rapid size- and shapecontrolled continuous hydrothermal synthesis of metal sulphide nanomaterials. Nanoscale 2014 $6(4), 2406-2418$

371. Sierra-Pallares, J.; Huddle, T.; Garcia-Serna, J.; Alonso, E.; Mato, F.; Shvets, I.; Luebben, O.; Cocero, M. J.; Lester, E., Understanding bottom-up continuous hydrothermal synthesis of nanoparticles using empirical measurement and computational simulation. Nano Res. 2016, 9 (11), 3377-3387.

372.http://cordis.europa.eu/docs/results/280/280g83/final1-final-public-report-for-shymanpublishable.pdf.

373. Sue, K.; Suzuki, A.; Hakuta, Y.; Hayashi, H.; Arai, K.; Takebayashi, Y.; Yoda, S.; Furuya, T., Hydrothermal-reduction synthesis of Ni nanoparticles by super rapid heating using a micromixer. Chem. Lett. 2009, 38 (11), 1018-1019.

374. Lubke, M.; Makwana, N. M.; Gruar, R.; Tighe, C.; Brett, D.; Shearing, P.; Liu, Z.; Darr, J. A., High capacity nanocomposite $\mathrm{Fe}_{3} \mathrm{O}_{4} / \mathrm{Fe}$ anodes for Li-ion batteries. J. Power Sources 2015, 291, 102107. 
375. Potter, D. B.; Bhachu, D. S.; Powell, M. J.; Darr, J. A.; Parkin, I. P.; Carmalt, C. J., Al-, Ga-, and Indoped $\mathrm{ZnO}$ thin films via aerosol assisted CVD for use as transparent conducting oxides. Phys. Status Solidi a-Appl.Mater.Sc. 2016, 213 (5), 1346-1352.

376. Li, H.; Arita, T.; Takami, S.; Adschiri, T., Rapid synthesis of tin-doped indium oxide microcrystals in supercritical water using hydrazine as reducing agent. Prog. Cryst. Growth Charact. Mater. 2011, $57(4), 117-126$.

377. Buhler, G.; Tholmann, D.; Feldmann, C., One-pot synthesis of highly conductive indium tin oxide nanocrystals. Adv. Mater. 2007, 19 (17), 2224-2227.

378. Fang, Z.; Assaaoudi, H.; Guthrie, R. I. L.; Kozinski, J. A., Continuous synthesis of tin and indium oxide nanoparticles in sub- and supercritical water. J. Am. Ceram. Soc. 2007, 90 (8), 2367-2371.

379. Lu, J.; Minami, K.; Takami, S.; Shibata, M.; Kaneko, Y.; Adschiri, T., Supercritical hydrothermal synthesis and in situ organic modification of indium tin oxide nanoparticles using continuous-flow reaction system. ACS Appl. Mater. Int. 2012, 4 (1), 351-354.

380. Elouali, S.; Bloor, L. G.; Binions, R.; Parkin, I. P.; Carmalt, C. J.; Darr, J. A., Gas sensing with nanoindium oxides $\left(\mathrm{In}_{2} \mathrm{O}_{3}\right)$ prepared via continuous hydrothermal flow synthesis. Langmuir 2012, 28 (3), 1879-1885.

381. Lu, J.; Minami, K.; Takami, S.; Shibata, M.; Kaneko, Y.; Adschiri, T. Co-Doping of Tin and Zinc into Indium Oxide Nanocrystals Using a Facile Hydrothermal Method. Chem. Select 2016, 1 (3), $518-523$

382. Ghanizadeh, S.; Peiris, T. A. N.; Jayathilake, D. S. Y.; Hutt, D. A.; Wijayantha, K. G. U.; Southee, D. J.; Conway, P. P.; Marchand, P.; Darr, J. A.; Parkin, I. P.; Carmalt, C. J., Dispersion and microwave processing of nano-sized ITO powder for the fabrication of transparent conductive oxides Ceram. Int. 2016, 42 (16) 18296-18302 
383. Anikeev, V., I, Hydrothermal synthesis of metal oxide nano- and microparticles in supercritical water. Russ. J. Phys. Chem. A 2011, 85 (3), 377-382.

384. Howard, D. P.; Marchand, P.; Johnson, I. D.; Carmalt, C. J.; Parkin, I. P.; Darr, J. A., Conducting aluminium and gallium doped zinc oxides; rapid optimisation and scale-up. J. Mater. Chem. A 2016, 4, 12774-12780.

385. Howard, D.; Marchand, P.; McCafferty, L.; Carmalt, C. J.; Parkin, I. P.; Darr, J. A., High-throughput continuous hydrothermal synthesis of conducting aluminium and gallium co-doped zinc oxides". J. Combi Chem. 2017 2016, accepted for publication jan 2017.

386.Scanlon, D. O., Defect engineering of $\mathrm{BaSnO}_{3}$ for high-performance transparent conducting oxide applications. Phys.Rev. B 2013, 87 (16), Article 161201(R).

387. Bhachu, D. S.; Scanlon, D. O.; Sankar, G.; Veal, T. D.; Egdell, R. G.; Cibin, G.; Dent, A. J.; Knapp, C. E.; Carmalt, C. J.; Parkin, I. P., Origin of High Mobility in Molybdenum-Doped Indium Oxide. Chem. Mater. 2015, 27 (8), 2788-2796.

388. Regoutz, A.; Egdell, R. G.; Morgan, D. J.; Palgrave, R. G.; Tellez, H.; Skinner, S. J.; Payne, D. J.; Watson, G. W.; Scanlon, D. O., Electronic and surface properties of Ga-doped $\ln _{2} \mathrm{O}_{3}$ ceramics. Appl. Surf. Sci. 2015, 349, 970-982.

389.Ganose, A. M.; Scanlon, D. O., Band gap and work function tailoring of $\mathrm{SnO}_{2}$ for improved transparent conducting ability in photovoltaics. J. Mater. Chem. C 2016, 4 (7), 1467-1475. 390.Lebens-Higgins, Z.; Scanlon, D. O.; Paik, H.; Sallis, S.; Nie, Y.; Uchida, M.; Quackenbush, N. F.; Wahila, M. J.; Sterbinsky, G. E.; Arena, D. A.; Woicik, J. C.; Schlom, D. G.; Piper, L. F. J., Direct observation of electrostatically driven band gap renormalization in a degenerate perovskite transparent conducting oxide. Phys. Rev. Lett. 2016, 116 (2), Article 027602. 
391. Kehoe, A. B.; Arca, E.; Scanlon, D. O.; Shvets, I. V.; Watson, G. W., Assessing the potential of Mgdoped $\mathrm{Cr}_{2} \mathrm{O}_{3}$ as a novel p-type transparent conducting oxide. J. Phys. Cond. Matter 2016, 28 (12), Article 125501.

392.Dixon, S. C.; Scanlon, D. O.; Carmalt, C. J.; Parkin, I. P., n-Type doped transparent conducting binary oxides: an overview. J. Mater. Chem. C 2016, 4 (29), 6946-6961.

393. Takami, S.; Hayakawa, R.; Wakayama, Y.; Chikyow, T., Continuous hydrothermal synthesis of nickel oxide nanoplates and their use as nanoinks for p-type channel material in a bottom-gate field-effect transistor. Nanotech. 2010, 21 (13), 1-4.

394.Dong, W. J.; Lv, Y.; Xiao, L. L.; Fan, Y.; Zhang, N.; Liu, X. Y., Bifunctional $\mathrm{MoO}_{3}-\mathrm{WO}_{3} / \mathrm{Ag} / \mathrm{MoO}_{3}-$ $\mathrm{WO}_{3}$ Films for Efficient ITO-Free Electrochromic Devices. ACS Appl. Mater. Int. 2016, 8 (49), $33842-33847$

395.Sun, S. B.; Lu, T.; Chang, X. T.; Hu, X.; Dong, L. H.; Yin, Y. S., Flexible electrochromic device based on $\mathrm{WO}_{3} \cdot \mathrm{H}_{2} \mathrm{O}$ nanoflakes synthesized by a facile sonochemical method. Mater. Lett. 2016, 185, $319-322$.

396. Bogati, S.; Georg, A.; Graf, W., Sputtered $\mathrm{Si}_{3} \mathrm{~N}_{4}$ and $\mathrm{SiO}_{2}$ electron barrier layer between a redox electrolyte and the $\mathrm{WO}_{3}$ film in electrochromic devices. Sol. Energy Mater. Sol. Cells 2017, 159, $395-404$.

397. Gimeno-Fabra, M.; Dunne, P.; Grant, D.; Gooden, P.; Lester, E., Continuous flow synthesis of tungsten oxide $\left(\mathrm{WO}_{3}\right)$ nanoplates from tungsten (VI) ethoxide. Chem. Eng. J. 2013, 226, 22-29.

398.Sänze, S.; Hess, C., Ethanol gas sensing by indium oxide: an operando spectroscopic raman-FTIR study. J. Phys. Chem. C 2014, $118(44), 25603-25613$.

399.Barsan, N.; Weimar, U., Conduction model of metal oxide gas sensors. J. Electroceram. 2001, 7 (3), $143-167$ 
400. Korotcenkov, G. C., A.; Brinzari, V.; Vasiliev, A.; Ivanov, M.; Cornet, A.; Morante, J.; Cabot, A.; Arbiol, , $\ln _{2} \mathrm{O}_{3}$ films deposited by spray pyrolysis as a material for ozone gas sensors. J. Sens. Actuators, B 2004, 99, 297-303.

401.Kaur, M.; Kailasaganapathi, S.; Ramgir, N.; Datta, N.; Kumar, S.; Debnath, A. K.; Aswal, D. K.; Gupta, S. K., Gas dependent sensing mechanism in ZnO nanobelt sensor. Appl. Surf. Sci. 2017, $394,258-266$.

402. Li, Y.; Jiao, M. F.; Yang, M. J., In-situ grown nanostructured $\mathrm{ZnO}$ via a green approach and gas sensing properties of polypyrrole/ZnO nanohybrids. Sens. Act. B-Chem. 2017, 238, 596-604. 403.Ahn, M. W.; Park, K. S.; Heo, J. H.; Kim, D. W.; Choi, K. J.; Park, J. G., On-chip fabrication of ZnOnanowire gas sensor with high gas sensitivity. Sens. Act. B-Chem 2009, 138 (1), 168-173.

404. Ahn, M. W.; Park, K. S.; Heo, J. H.; Park, J. G.; Kim, D. W.; Choi, K. J.; Lee, J. H.; Hong, S. H., Gas sensing properties of defect-controlled ZnO-nanowire gas sensor. Appl. Phys. Lett. 2008, 93 (26), Article 263103

405. Jung, J. H.; Lee, M.; Hong, J. I.; Ding, Y.; Chen, C. Y.; Chou, L. J.; Wang, Z. L. Lead-Free NaNbO 3 Nanowires for a High Output Piezoelectric Nanogenerator. ACS Nano. 2011, 5 (12), 10041-10046 406. Xu, S.; Qin, Y.; Xu, C.; Wei, Y. G.; Yang, R. S.; Wang, Z. L., Self-powered nanowire devices. Nature Nanotech. 2010, 5 (5), 366-373.

407. Ahn, C. W.; Hong, C. H.; Choi, B. Y.; Kim, H. P.; Han, H. S.; Hwang, Y.; Jo, W.; Wang, K.; Li, J. F.; Lee, J. S.; Kim, I. W., A brief review on relaxor ferroelectrics and selected issues in lead-free relaxors. J. Kor. Phys.Soc. 2016, 68 (12), 1481-1494.

408. Meena, J. S.; Sze, S. M.; Chand, U.; Tseng, T. Y., Overview of emerging nonvolatile memory technologies. Nano. Res. Lett. 2014, 9 (526), 1-33.

409. Fiebig, M.; Lottermoser, T.; Meier, D.; Trassin, M., The evolution of multiferroics. Nature Rev. Mater. 2016, 1 (8), Article 16046. 
410.Hsu, C. H.; Soong, H. T.; Yu, C. C.; Huang, C. L.; Ku, M. T., The effect of sintering temperature and time on microwave properties of $\mathrm{Sm}\left(\mathrm{Zn}_{1 / 2} \mathrm{Ti}_{1 / 2}\right) \mathrm{O}_{3}$ ceramics for resonators. Ceram. Int. 2007, 33 (6), 951-955.

411.Dernovsek, O.; Naeini, A.; Preu, G.; Wersing, W.; Eberstein, M.; Schiller, W. A., LTCC glassceramic composites for microwave application. J. Eur. Ceram. Soc. 2001, 21 (10-11), 1693-1697.

412.Takada, T.; Wang, S. F.; Yoshikawa, S.; Jang, S. J.; Newnham, R. E., Effects of glass additions on (Zr,Sn)TiO 4 for microwave applications. J. Am. Ceram. Soc. 1994, 77 (9), 2485-2488.

413. Kagata, H.; Inoue, T.; Kato, J.; Kameyama, I., Low-fire bismuth-based dielectric ceramics for microwave use. Jap. J. Appl. Phys. Part 1-Reg.Pap. Short Notes Rev. Pap. 1992, 31 (9B), 3152-3155. 414. Gajbhiye, N. S.; Pandey, P. K.; George, L.; Kumar, A., Characterization of nanostructured PZT prepared by chemical routes. J. Nanosci. Nanotech. 2007, 7 (6), 1975-1979.

415. Gritzner, G.; Pissenberger, A., Dielectric properties of $\mathrm{Bi}_{2} \mathrm{PbNb}_{2} \mathrm{O}_{9}$ ceramics prepared by different techniques. Appl. Phys. A-Mater. Sci. Proc. 2007, 87 (4), 807-811.

416.Shepherd, P.; Mallick, K. K.; Green, R. J., Dielectric properties of cobalt substituted M-type barium hexaferrite prepared by co-precipitation. J. Mater. Sci.-Mater. Elec. 2007, 18 (5), 527-534. 417. Hou, Y. D.; Hou, L.; Zhu, M. K.; Wang, H.; Yan, H., Sol-gel synthesis of lead-free $\left(\mathrm{K}_{0.5} \mathrm{Bi}_{0.5}\right)_{0.4} \mathrm{Ba}_{0.6} \mathrm{TiO}_{3}$ nanorods. Mater. Lett. 2007, 61 (16), 3371-3373.

418. Cwikiel, K.; Nogas-Cwikiel, E., Dielectric and pyroelectric properties of the $\mathrm{Ba}_{0.3} \mathrm{Na}_{0.7} \mathrm{Ti}_{0.3} \mathrm{Nb}_{0.7} \mathrm{O}_{3}$ ceramics obtained by sol-gel method. Phase Trans 2007, 80 (1-2), 141-146.

419.Xu, J. B.; Zhai, J. W.; Yao, X.; Xue, J. Q.; Huang, Z. M., Dielectric and optical properties of BaTiO 3 thin films prepared by low-temperature process. J. Sol-Gel Sci. Technol. 2007, 42 (3), 209-212.

420. Kolen'ko, Y. V.; Kovnir, K. A.; Neira, I. S.; Taniguchi, T.; Ishigaki, T.; Watanabe, T.; Sakamoto, N.; Yoshimura, M., A novel, controlled, and high-yield solvothermal drying route to nanosized barium titanate powders. J. Phys. Chem. C 2007, 111 (20), 7306-7318. 
421.Pramanik, N. C.; Seok, S. I.; Ahn, B. Y., Wet-chemical synthesis of crystalline $\mathrm{BaTiO}_{3}$ from stable chelated titanium complex: Formation mechanism and dispersibility in organic solvents. J. Coll. Int. Sci. 2006, $300(2), 569-576$.

422. Suzuki, K.; Kijima, K., Effect of oxygen injection on synthesizing barium titanate nanoparticles by plasma chemical vapor deposition. J. Mater. Sci. 2006, 41 (16), 5346-5358.

423. Bocquet, J. F.; Chhor, K.; Pommier, C., Barium titanate powders synthesis from solvothermal reaction and supercritical treatment. Mater. Chem. Phys. 1999, 57 (3), 273-280.

424. Reveron, H.; Elissalde, C.; Aymonier, C.; Bousquet, C.; Maglione, M.; Cansell, F., Continuous supercritical synthesis and dielectric behaviour of the whole BST solid solution. Nanotech. 2006, $17(14), 3527-3532$.

425.Philippot, G.; Boejesen, E. D.; Elissalde, C.; Maglione, M.; Aymonier, C.; Iversen, B. B., Insights into $\mathrm{BaTi}_{1-\mathrm{y}} \mathrm{Zr}_{\mathrm{y}} \mathrm{O}_{3}$ (o ilippotsynthesis under supercritical fluid conditions. Chem. Mater. 2016, 28 (10), 3391-3400.

426. Philippot, G.; Albino, M.; Chung, U. C.; Josse, M.; Elissalde, E.; Maglione, M.; Aymonier, C., Continuous $\mathrm{BaTi}_{1-\mathrm{y}} \mathrm{Zr}_{\mathrm{y}} \mathrm{O}_{3}(\mathrm{o} \leq \mathrm{y} \leq 1)$ nanocrystals synthesis in Supercrit. Fluids for nanostructured lead-free ferroelectric ceramics. Mater. Des. 2015, 86, 354-36o.

427. Matsui, K.; Noguchi, T.; Islam, N. M.; Hakuta, Y.; Hayashi, H., Rapid synthesis of $\mathrm{BaTiO}_{3}$ nanoparticles in supercritical water by continuous hydrothermal flow reaction system. J. Cryst. Growth 2008, 310 (10), 2584-2589.

428. Hayashi, H.; Noguchi, T.; Islam, N. M.; Hakuta, Y.; Imai, Y.; Ueno, N., Hydrothermal synthesis of $\mathrm{BaTiO}_{3}$ nanoparticles using a supercritical continuous flow reaction system. J. Cryst. Growth 2010, $312(12-13), 1968-1972$. 
429. Hayashi, H.; Noguchi, T.; Islam, N. M.; Hakuta, Y.; Imai, Y.; Ueno, N., Hydrothermal synthesis of organic hybrid $\mathrm{BaTiO}_{3}$ nanoparticles using a supercritical continuous flow reaction system. J. Cryst. Growth 2010, $312(24), 3613-3618$.

430.Dunne, P. W.; Starkey, C. L.; Munn, A. S.; Tang, S. V. Y.; Luebben, O.; Shvets, I.; Ryder, A. G.; Casamayou-Boucau, Y.; Morrison, L.; Lester, E. H., Bench- and pilot-scale continuous-flow hydrothermal production of barium strontium titanate nanopowders. Chem. Eng. J. 2016, 289, $433-441$.

431. Araki, S.; Yamamoto, H.; Hoshi, Y.; Lu, J.; Hakuta, Y.; Hayashi, H.; Ohashi, T.; Sato, K.; Nishioka, M.; Inoue, T.; Hikazudani, S.; Hamakawa, S., Synthesis of $\mathrm{Ca}_{0.8} \mathrm{Sr}_{0.2} \mathrm{Ti}_{0.7} \mathrm{Fe}_{0.3} \mathrm{O}_{3-\mathrm{d}}$ thin film membranes and its application to the partial oxidation of methane. Solid State lonics 2012, 221, 43-49.

432. Lu, J.; Hakuta, Y.; Hayashi, H.; Ohashi, T.; Hoshi, Y.; Sato, K.; Nishioka, M.; Inoue, T.; Hamakawa, S., Continuous hydrothermal preparation of partially substituted perovskite oxide nanoparticles. Chem. Lett. 2007, 36 (10), 1262-1263.

433. Lu, J.; Hakuta, Y.; Hayashi, H.; Ohashi, T.; Nagase, T.; Hoshi, Y.; Sato, K.; Nishioka, M.; Inoue, T.; Hamakawa, S., Preparation of $\mathrm{Ca}_{0.8} \mathrm{Sr}_{0.2} \mathrm{Ti}_{1-x} \mathrm{Fe}_{x} \mathrm{O}_{3-\mathrm{d}}(\mathrm{x}=0.1-0.3)$ nanoparticles using a flow supercritical reaction system. J. Supercrit. Fluids 2008, 46 (1), 77-82.

434. Fukuda, K.; Kitoh, R.; Awai, I., Microwave characteristics of $\mathrm{TiO}_{2}-\mathrm{Bi}_{2} \mathrm{O}_{3}$ dielectric resonator. Jpn. J. Appl. Phys. 1993, 32(10), 4584-4588.

435. Gongora-Rubio, M. R.; Espinoza-Vallejos, P.; Sola-Laguna, L.; Santiago-Aviles, J. J., Overview of low temperature co-fired ceramics tape technology for meso-system technology (MsST). Sens. Act. A-Phys. 2001, 89 (3), 222-241.

436.Kuang, X.; Carotenuto, G.; Nicolais, L., A review of ceramic sintering and suggestions on reducing sintering temperatures. Adv. Perf. Mater. 1997, 4 (3), 257-274. 
437. Tokura, Y., Multiferroics as quantum electromagnets. Science 2006, 312 (5779), 1481-1482.

438.Philippot, G.; Elissalde, C.; Maglione, M.; Aymonier, C., Supercritical fluid technology: A reliable process for high quality $\mathrm{BaTiO}_{3}$ based nanomaterials. Adv. Powder Technol. 2014, 25 (5), 14151429.

439.Philippot, G.; Albino, M.; Epherre, R.; Chevallier, G.; Beynet, Y.; Manière, C.; Weibel, A.; Peigney, A.; Deluca, M.; Elissalde, C.; Maglione, M.; Aymonier, C.; Estournès, C., Local distortions in nanostructured ferroelectric ceramics through strain tuning. Adv. Elec. Mater. 2010, 1 (10), Article 1500190.

440. Maglione, M.; Philippot, G.; Levasseur, D.; Payan, S.; Aymonier, C.; Elissalde, C., Defect chemistry in ferroelectric perovskites: long standing issues and recent advances. Dalton Trans., 2015, 44 (30), 13411-13418.

441. Hayashi, H.; Hakuta, Y.; Kurata, Y., Hydrothermal synthesis of potassium niobate photocatalysts under subcritical and supercritical water conditions. J. Mater. Chem. 2004, 14 (13), 2046-2051.

442. Philippot, G.; Jensen, K. M.; Christensen, M.; Elissalde, C.; Maglione, M.; Iversen, B. B.; Aymonier, C., Coupling in situ synchrotron radiation with ex situ spectroscopy characterizations to study the formation of $\mathrm{Ba}_{1-x} \mathrm{Sr}_{x} \mathrm{TiO}_{3}$ nanoparticles in Supercrit. Fluids. J. Supercrit. Fluids 2014, $87,111-117$.

443.Mi, J. L.; Jensen, T. N.; Christensen, M.; Tyrsted, C.; Jorgensen, J. E.; Iversen, B. B., Hightemperature and high-pressure aqueous solution formation, growth, crystal structure, and

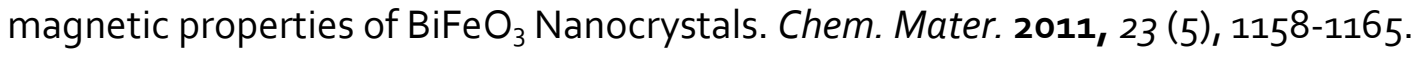

444. Takami, S.; Sato, T.; Mousavand, T.; Ohara, S.; Umetsu, M.; Adschiri, T., Hydrothermal synthesis of surface-modified iron oxide nanoparticles. Mater. Lett. 2007, 61 (26), 4769-4772.

445. Darab, J. G.; Matson, D. W., Continuous hydrothermal processing of nano-crystalline particulates for chemical-mechanical planarization. J. Electron. Mater. 1998, 27 (10), 1068-1072. 
446. Hao, Y. L.; Teja, A. S., Continuous hydrothermal crystallization of alpha- $\mathrm{Fe}_{2} \mathrm{O}_{3}$ and $\mathrm{CO}_{3} \mathrm{O}_{4}$ nanoparticles. J. Mater. Res. 2003, 18 (2), 415-422.

447.Becker, J.; Lock, N.; Bremholm, M.; Tyrsted, C.; Pauw, B.; Jensen, K. M. Ø.; Eltzholtz, J. R.; Christensen, M.; Iversen, B. B., Peering into the Black Box: In situ X Ray SAXS/WAXS/PDF studies of the formation and growth of inorganic nanoparticles during Near or supercritical synthesis. Int. IAEA Rad. Tech. Rep. no. 4 2015, 46 (21), Article 46048649.

448. Hojo, D.; Suzuki, K. Z.; Mizukami, S.; Adschiri, T., Magneto-optical Kerr effect characterization of a uniform nanocrystalline $\mathrm{Fe}_{3} \mathrm{O}_{4}$ monolayer fabricated on a silicon substrate functionalized with catechol groups. J. Mater. Chem. C 2016, 4 (6), 1263-1270.

449. Nilsen, M. H.; Nordhei, C.; Ramstad, A. L.; Nicholson, D. G.; Poliakoff, M.; Cabanas, A., XAS (XANES and EXAFS) investigations of nanoparticulate ferrites synthesized continuously in near critical and supercritical water. J. Phys. Chem. C 2007, 111 (17), 6252-6262.

450.Cote, L. J.; Teja, A. S.; Wilkinson, A. P.; Zhang, Z. J., Continuous hydrothermal synthesis of $\mathrm{CoFe}_{2} \mathrm{O}_{4}$ nanoparticles. Fluid Phase Equilib. 2003, 210 (2), 307-317.

451. Millot, N.; Le Gallet, S.; Aymes, D.; Bernard, F.; Grin, Y., Spark plasma sintering of cobalt ferrite nanopowders prepared by coprecipitation and hydrothermal synthesis. J. Eur. Ceram. Soc. 2007, $27(2-3), 921-926$.

452.Sato, T.; Sue, K.; Suzuki, W.; Suzuki, M.; Matsui, K.; Hakuta, Y.; Hayashi, H.; Arai, K.; Kawasaki, S. I.; Kawai-Nakamura, A.; Hiaki, T., Rapid and continuous production of ferrite nanoparticles by hydrothermal synthesis at $673 \mathrm{~K}$ and $30 \mathrm{MPa}$. Ind. Eng. Chem. Res. 2008, 47 (6), 1855-1860.

453. Sue, K.; Aoki, M.; Sato, T.; Nishio-Hamane, D.; Kawasaki, S. I.; Hakuta, Y.; Takebayashi, Y.; Yoda, S.; Furuya, T.; Sato, T.; Hiaki, T., Continuous hydrothermal synthesis of nickel ferrite nanoparticles using a central collision-type micromixer: effects of temperature, residence time, 
metal salt molality, and $\mathrm{NaOH}$ addition on conversion, particle size, and crystal phase. Ind. Eng. Chem. Res. 2011, 50 (16), 9625-9631.

454. Nam, S. C.; Kim, G. J., Characterization of barium hexaferrite produced by varying the reaction parameters at the mixing-points in a supercritical water crystallization process. Korean J. Chem. Eng. 2004, 21 (3), 582-588.

455. Rho, S.; Park, S., Influence of stoichiometry and alkalinity on barium hexaferrite formation via the supercritical water crystallization method. Korean J. Chem. Eng. 2002, 19 (1), 120-125.

456. Rangappa, D.; Naka, T.; Ohara, S.; Adschiri, T., Preparation of Ba-hexaferrite nanocrystals by an organic ligand-assisted supercritical water process. Cryst. Grow. Des. 2010, 10 (1), 11-15.

457. Bernardi, S.; Adid Adep, D.; Jae-Wook, C.; Jeong-Myeong, H.; Dong Jin, S.; Chang SoO, K.; JongChoo, L., Catalytic depolymerization of alkali lignin using supported Pt nanoparticle catalysts J. Nanosci. Nanotech. 2016, 16 (5), 4570-4575.

458. Ao, C. H.; Lee, S. C., Indoor air purification by photocatalyst $\mathrm{TiO}_{2}$ immobilized on an activated carbon filter installed in an air cleaner. Chem. Eng. Sci. 2005, 60 (1), 103-109.

459. Alinsafi, A.; Evenou, F.; Abdulkarim, E. M.; Pons, M. N.; Zahraa, O.; Benhammou, A.; Yaacoubi, A.; Nejmeddine, A., Treatment of textile industry wastewater by supported photocatalysis. Dyes and Pigments 2007, 74 (2), 439-445.

46o. Fujishim, A.; Honda, K., Electrochemical photolysis of water at a semiconductor electrode. Nature 1972, $238(5358), 37-38$.

461. Yang, J. H.; Han, Y. S.; Choy, J. H., $\mathrm{TiO}_{2}$ thin-films on polymer substrates and their photocatalytic activity. Thin Solid Films 2006, 495 (1-2), 266-271.

462. Dunnill, C. W.; Aiken, Z. A.; Pratten, J.; Wilson, M.; Parkin, I. P., N-doped titania thin films, prepared by atmospheric pressure chemical vapour deposition: enhanced visible light 
photocatalytic activity and anti-microbial effects. In Eurocvd 17 / Cvd 17, Swihart, M. T.; Barreca, D.; Adomaitis, R. A.; Worhoff, K., Eds. 2009; 25 (8), 65-72.

463. Elouali, S.; Mills, A.; Parkin, I. P.; Bailey, E.; McMillan, P. F.; Darr, J. A., Photocatalytic evolution of hydrogen and oxygen from ceramic wafers of commercial titanias. J. Photochem. Photobiol. AChem. 2010, 216 (2-3), 110-114.

464. Kafizas, A.; Crick, C.; Parkin, I. P., The combinatorial atmospheric pressure chemical vapour deposition (CAPCVD) of a gradating substitutional/interstitial N-doped anatase $\mathrm{TiO}_{2}$ thin-film; UVA and visible light photocatalytic activities. J. Photochem. Photobiol. A-Chem. 2010, 216 (2-3), $156-166$.

465. Edusi, C.; Hyett, G.; Sankar, G.; Parkin, I. P., Aerosol-assisted CVD of titanium dioxide thin films from methanolic solutions of titanium tetraisopropoxide; substrate and aerosol-selective deposition of rutile or anatase. Chem. Vap. Dep. 2011, 17 (1-3), 30-36.

466. Cabrera, R. Q.; Latimer, E. R.; Kafizas, A.; Blackman, C. S.; Carmalt, C. J.; Parkin, I. P., Photocatalytic activity of needle-like $\mathrm{TiO}_{2} / \mathrm{WO}_{3-x}$ thin films prepared by chemical vapour deposition. J. Photochem. Photobiol. A-Chem.2012, 239, 60-64.

467.Powell, M. J.; Dunnill, C. W.; Parkin, I. P., N-doped $\mathrm{TiO}_{2}$ visible light photocatalyst films via a solgel route using TMEDA as the nitrogen source. J. Photochem. Photobiol. A-Chem. 2014, 281, 27-34. 468. Powell, M. J.; Palgrave, R. G.; Dunnill, C. W.; Parkin, I. P., A fast and effective method for Ndoping $\mathrm{TiO}_{2}$ by post treatment with liquid ammonia: visible light photocatalysis. Thin Solid Films $2014,562,223-228$

469. Alotaibi, A. M.; Sathasivam, S.; Parkin, I. P., Aerosol assisted chemical vapour deposition of a $\mathrm{ZrO}_{2}-\mathrm{TiO}_{2}$ composite thin film with enhanced photocatalytic activity. RSC Adv. 2015, 5 (83), $67944-67950$. 
470. Fan, Y.; Chen, G.; Li, D.; Luo, Y.; Lock, N.; Jensen, A. P.; Mamakhel, A.; Mi, J.; Iversen, S. B.; Meng, Q.; Iversen, B. B., Highly selective deethylation of rhodamine $\mathrm{B}$ on $\mathrm{TiO}_{2}$ prepared in supercrit. fluids. Int. J. Photoen. 2012, 2012 1-7.

471. Yang, Y.; Lin, T.; Weng, X. L.; Darr, J. A.; Wang, X. Z., Data flow modeling, data mining and QSAR in high-throughput discovery of functional nanomaterials. Comp. Chem. Eng. 2011, 35 (4), 671678.

472. Dunne, P. W.; Starkey, C. L.; Munn, A. S.; Sikder, M.; Luebben, O.; Shvets, I.; Lester, E. H., Transition metal doped anatase nanocrystals: continuous-flow hydrothermal synthesis and photocatalytic activity. J. Envir.Chem. Eng. 2016, 4 (3), 2665-2670.

473. Zhang, Z.; Goodall, J. B. M.; Morgan, D. J.; Brown, S.; Clark, R. J. H.; Knowles, J. C.; Mordan, N. J.; Evans, J. R. G.; Carley, A. F.; Bowker, M.; Darr, J. A., Photocatalytic activities of N-doped nanotitanias and titanium nitride. J. Eur. Ceram. Soc. 2009, 29 (11), 2343-2353.

474. Jeon, J. W.; Kim, J. R.; Ihm, S. K., Continuous one-step synthesis of N-doped titania under supercritical and subcritical water conditions for photocatalytic reaction under visible light. J. Phys. Chem. Solids 2010, 71 (4), 608-611.

475. Zhang, Z.; Goodall, J. B.; Brown, S.; Karlsson, L.; Clark, R. J.; Hutchison, J. L.; Rehman, I.; Darr, J. A., Continuous hydrothermal synthesis of extensive $2 \mathrm{D}$ sodium titanate $\left(\mathrm{Na}_{2} \mathrm{Ti}_{3} \mathrm{O}_{7}\right)$ nano-sheets. Dalton Trans. 2010, 39 (3), 711-714.

476. Hayashi, H.; Nakamura, T.; Ebina, T., Hydrothermal synthesis of sodium titanate nanosheets using a supercritical flow reaction system. J. Ceram. Soc. Jpn. 2016, 124 (1), 74-78.

477. Pirkanniemi, K.; Sillanpaa, M., Heterogeneous water phase catalysis as an environmental application: a review. Chemosphere 2002, 48 (10), 1047-1060.

478. Wen, F. S.; Li, W. L.; Moon, J. H.; Kim, J. H., Hydrothermal synthesis of ZnO : Zn with green emission at low temperature with reduction process. Solid State Commun. 2005, 135 (1-2), 34-37. 
479.Xu, P.; Wen, X.; Zheng, Z.; Cox, G.; Zhu, H., Two-photon optical characteristics of zinc oxide in bulk, low dimensional and nanoforms. J. Lumin. 2007, 126 (2), 641-643.

480. Viswanathan, R.; Gupta, R. B., Formation of zinc oxide nanoparticles in supercritical water. J. Supercrit. Fluids 2003, 27 (2), 187-193.

481. Viswanathan, R.; Lilly, G. D.; Gale, W. F.; Gupta, R. B., Formation of zinc oxide-titanium dioxide composite nanoparticles in supercritical water. Ind. Eng. Chem. Res. 2003, 42 (22), 5535-5540.

482. Kudo, A., Development of photocatalyst materials for water splitting. Int. J. Hydrogen Energy 2006, $31(2), 197-202$.

483.ISO standard Method for air-purification performance of semiconducting photocatalytic materials. Removal of nitric oxide (ISO 22197-1:2007) 2007. www.iso.org/iso/iso_catalogue/catalogue_tc/catalogue_detail.htm?csnumber=40761].

484. "High throughput Nanoceramics Discovery Project" funded by EPSRC (reference EP/Do38499/2) summary available at http://gow.epsrc.ac.uk/ViewGrant.aspx?GrantRef=EP/Do38499/2.

485. Houlberg, K.; Bøjesen, E. D.; Tyrsted, C.; Mamakhel, A.; Wang, X.; Su, R.; Besenbacher, F.; Iversen, B. B., Hydrothermal synthesis and in situ powder X-ray diffraction study of bismuthsubstituted ceria nanoparticles. Cryst. Grow. Des. 2015, 15 (8), 3628-3636.

486. Slavinskaya, E. M.; Kardash, T. Y.; Stonkus, O. A.; Gulyaev, R. V.; Lapin, I. N.; Svetlichnyi, V. A.; Boronin, A. I., Metal-support interaction in $\mathrm{Pd} / \mathrm{CeO}_{2}$ model catalysts for $\mathrm{CO}$ oxidation: from pulsed laser-ablated nanoparticles to highly active state of the catalyst. Catal. Sci. Tech.. 2016, 6 (17), 6650-6666.

487. Ivanovaa, A. S.; Slavinskayaa, E. M.; Gulyaeva, R. V.; Zaikovskiia, V. I.; Stonkusb, O. A.; Danilovaa, I. G.; Plyasovaa, L. M.; Polukhinaa, I. A.; Boronina, A. I., Metal-support interactions in Pt/AI ${ }_{2} \mathrm{O}_{3}$ and $\mathrm{Pd} / \mathrm{Al}_{2} \mathrm{O}_{3}$ catalysts for $\mathrm{CO}$ oxidation. Appl. Cat. B: Envir.2010, 97 (1-2), 57-71. 
488. Wang, T.; Smith, R. L.; Inomata, H.; Arai, K., Reactive phase behavior of aluminum nitrate in high temperature and supercritical water. Hydromet. 2002, 65 (2-3), 159-175.

489. Sato, T.; Sue, K.; Akiyama, Y.; Shibata, K.; Kawasaki, S. i.; Tanaka, S.; Saitoh, K.; KawaiNakamura, A.; Aida, K.; Hiaki, T., Effect of pH on hydrothermal synthesis of gamma- $\mathrm{Al}_{2} \mathrm{O}_{3}$ nanoparticles at 673 K. Chem. Lett. 2008, 37 (3), 242-243.

490. Noguchi, T.; Matsui, K.; Islam, N. M.; Hakuta, Y.; Hayashi, H., Rapid synthesis of gamma- $\mathrm{Al}_{2} \mathrm{O}_{3}$ nanoparticles in supercritical water by continuous hydrothermal flow reaction system. J. Supercrit. Fluids 2008, 46 (2), 129-136.

491. Hakuta, Y.; Adschiri, T.; Hirakoso, H.; Arai, K., Chemical equilibria and particle morphology of boehmite $(\mathrm{AlOOH})$ in sub and supercritical water. Fluid Phase Equilib. 1999, 158-160, 733-742.

492. Lock, N.; Hald, P.; Christensen, M.; Birkedal, H.; Iversen, B. B., Continuous flow supercritical water synthesis and crystallographic characterization of anisotropic boehmite nanoparticles. J. Appl. Crystallogr. 2010, 43, 858-866.

493.Wang, J. H.; Chen, H.; Hu, Z. C.; Yao, M. F.; Li, Y. D., A review on the Pd-based three-way catalyst. Catal. Rev.-Sci. Eng. 2015, 57 (1), 79-144.

494. Poggiani, C.; Cimarello, A.; Grimaldi, C. N., Optimization of a fast light-off exhaust system for motorcycle applications. In 7oth Conference of the Italian Thermal Machines Engineering Association (Ati2015), Corsini, A.; Rispoli, F.; DeSantoli, L., Eds. Elsevier Science Amsterdam, 2015; Vol. 82, pp 75-80.

495. He, J. J.; Wang, C. X.; Zheng, T. T.; Zhao, Y. K., Thermally induced deactivation and the corresponding strategies for improving durability in automotive three-way catalysts. JM Tech. Rev. 2016, 60 (3), 196-203.

496. Montini, T.; Melchionna, M.; Monai, M.; Fornasiero, P., Fundamentals and catalytic applications of $\mathrm{CeO}_{2}$-based materials. Chem. Rev. 2016, 116 (10), 5987-6041. 
497. Cabanas, A.; Darr, J. A.; Lester, E.; Poliakoff, M., A continuous and clean one-step synthesis of nano-particulate $\mathrm{Ce}_{1-x} \mathrm{Zr}_{x} \mathrm{O}_{2}$ solid solutions in near-critical water. Chem. Commun., 2000, 901-902

498. Weng, X.; Boldrin, P.; Zhang, Z.; Darr, J., Continuous hydrothermal flow syntheses of nanosized energy materials used in solid oxide fuel cells. Clean Tech. 2008: Bio Energy, Renew., Green Build., Smart Grid, Stor Water 2008, 261-264.

499. Kim, K. H.; Kim, J. R.; Ihm, S. K., Wet oxidation of phenol over transition metal oxide catalysts supported on $\mathrm{Ce}_{0.65} \mathrm{Zr}_{0.35} \mathrm{O}_{2}$ prepared by continuous hydrothermal synthesis in supercritical water. J. Hazard. Mater. 2009, 167 (1-3), 1158-1162.

500.Liu, Y.; Yao, W.; Cao, X.; Weng, X.; Wang, Y.; Wang, H.; Wu, Z., Supercritical water syntheses of $\mathrm{Ce}_{x} \mathrm{TiO}_{2}$ nano-catalysts with a strong metal-support interaction for selective catalytic reduction of $\mathrm{NO}$ with $\mathrm{NH}_{3}$. Appl. Cat. B: Envir.2014, 160-161, 684-691.

501. Sundermann, A.; Gerlach, O., High-throughput screening as a supplemental tool for the development of advanced emission control catalysts: methodological approaches and data processing. Catalysts 2016, 6 (2), Article 23.

502.Lombardi, J.; Pearsall, F.; Libc, W.; O'Brien, S., Synthesis and dielectric properties of nanocrystalline oxide perovskites, $\left[\mathrm{KNbO}_{3}\right]_{1-x}\left[\mathrm{BaNi}_{0.5} \mathrm{Nb}_{0.5} \mathrm{O}_{3-\delta}\right]_{x}$, derived from potassium niobate $\mathrm{KNbO}_{3}$ by gel collection J. Mater. Chem. C 2016, 4, 7989-7998.

503. Meng, O.; Wang, W.; Weng, X.; Liu, Y.; Wang, H.; Wu, Z., Active Oxygen Species in $\mathrm{La}_{n+1} \mathrm{Ni}_{n} \mathrm{O}_{3 n+1}$ Layered Perovskites for Catalytic Oxidation of Tolvene and Methane. J. Phys. Chem. C 2016, 120 (6), 3259-3266.

504.Islam, N. M.; Noguchi, T.; Hakuta, Y.; Hayashi, H., Hydrothermal synthesis of strontium doped lanthanum manganite nanoparticles by a supercritical flow reaction system. Nanosci. Nanotech. Letts. 2011, 3 (3), 324-327. 
505.Wang, W. L.; Meng, Q.; Weng, X.; Wu, Z., Rapid syntheses of ultrafine $\mathrm{LaMnO}_{3}$ nano-crystallites with superior activity for catalytic oxidation of toluene. Catal. Commun. 2016, 84, 167-170.

506. Zhang, J.; Weng, X.; Wu, Z.; Liu, Y.; Wang, H., Facile synthesis of highly active $\mathrm{LaCoO}_{3} / \mathrm{MgO}$ composite perovskite via simultaneous co-precipitation in supercritical water. Appl. Cat. B: Envir.2012, 126, 231-238.

507.Zhang, J.; Tan, D.; Meng, Q.; Weng, X.; Wu, Z., Structural modification of $\mathrm{LaCoO}_{3}$ perovskite for oxidation reactions: the synergistic effect of $\mathrm{Ca}^{2+}$ and $\mathrm{Mg}^{2+} \mathrm{co}-$ substitution on phase formation and catalytic performance. Appl. Cat. B: Envir.2015, 172, 18-26.

508.Sun, S. M.; Pang, G. S.; Huang, Y. L.; Li, C. G.; Feng, S. H., Preparation of Well-Dispersed Mgdoped $\mathrm{LaCoO}_{3}$ nanocrystals with controllable particle size and high visible-light photocatalytic activity. J. Nanosci. Nanotech. 2010, 10 (8), 5207-5212.

509. Schulz, H., Short history and present trends of Fischer-Tropsch synthesis. Applied Catalysis AGeneral 1999, $186(1-2), 3-12$.

510. Sadasivan, S.; Bellabarba, R. M.; Tooze, R. P., Size dependent reduction-oxidation-reduction behaviour of cobalt oxide nanocrystals. Nanoscale 2013, 5, 11139 - 11146.

511. Darab, J. G.; Linehan, J. C.; Matson, D. W., Effect of agglomerate size on the catalytic activity of an iron oxyhydroxide nanocrystalline powder toward carbon-carbon bond scission in naphthylbibenzylmethane. Energy \& Fuels 1994, 8 (4), 1004-1005.

512. Rhodes, C. J., Properties and applications of zeolites. Sci. Prog. 2010, 93 (3), 223-284.

513. Fan, W.; Ogura, M.; Sankar, G.; Okubo, T., In situ small-angle and wide-angle X-ray scattering investigation on nucleation and crystal growth of nanosized zeolite A. Chem. Mater. 2007, 19 (8), 1906-1917. 
514. Walton, R. I.; Millange, F.; O'Hare, D.; Davies, A. T.; Sankar, G.; Catlow, C. R. A., An in situ energydispersive X-ray diffraction study of the hydrothermal crystallization of zeolite A. 1. Influence of reaction conditions and transformation into sodalite. J. Phys. Chem. B 2001, 105 (1), 83-90.

515. Liu, Z.; Okabe, K.; Anand, C.; Yonezawa, Y.; Zhu, J.; Yamada, H.; Endo, A.; Yanaba, Y.;

Yoshikawa, T.; Ohara, K.; Okubo, T.; Wakihara, T., Continuous flow synthesis of ZSM-5 zeolite on the order of seconds. PNAS 2016, $113(50), 14267-14271$.

516. Gimeno-Fabra, M.; Munn, A. S.; Stevens, L. A.; Drage, T. C.; Grant, D. M.; Kashtiban, R. J.; Sloan, J.; Lester, E.; Walton, R. I., Instant MOFs: continuous synthesis of metal-organic frameworks by rapid solvent mixing. Chem. Commun. 2012, $48(86), 10642-10644$.

517. Wang, W.; Wang, S.; Ma, X.; Gong, J., Chem. Soc. Rev. 2011, 40, 3703-3727.

518. Adeleyea, A. I.; Kellici, S.; Heil, T.; Morgan, D.; Vickers, M.; Saha, B., Greener synthesis of propylene carbonate using graphene-inorganic nanocomposite catalysts. Catal. Today 2015, 256, $347-357$

519. Roldan, A.; Hollingsworth, N.; Roffey, A.; Islam, H. U.; Goodall, J. B. M.; Catlow, C. R. A.; Darr, J. A.; Bras, W.; Sankar, G.; Holt, K. B.; Hogarth, G.; de Leeuw, N. H., Bio-inspired $\mathrm{CO}_{2}$ conversion by iron sulfide catalysts under sustainable conditions Chem. Commun. 2015, 51 (35), 7501-7504. 520.Le, M. T.; Do, V. H.; Truong, D. D.; Bruneel, E.; Van Driessche, I.; Riisager, A.; Fehrmann, R.; Trinh, Q. T., Synergy effects of the mixture of bismuth molybdate catalysts with $\mathrm{SnO}_{2} / \mathrm{ZrO}_{2} / \mathrm{MgO}$ in selective propene oxidation and the connection between conductivity and catalytic activity. Ind. Eng. Chem. Res. 2016, 55 (17), 4846-4855.

521. Thanh-Binh, N.; Dubois, J. L.; Kaliaguine, S., Ammoxidation of acrolein to acrylonitrile over bismuth molybdate catalysts. Applied Catalysis A-General 2016, 520, 7-12. 
522.Gruar, R.; Tighe, C. J.; Reilly, L. M.; Sankar, G.; Darr, J. A., Tunable and rapid crystallisation of phase pure $\mathrm{Bi}_{2} \mathrm{MoO}_{6}$ (koechlinite) and $\mathrm{Bi}_{2} \mathrm{Mo}_{3} \mathrm{O}_{12}$ via continuous hydrothermal synthesis. Solid State Sci.2010, 12 (9), 1683-1686.

523. Buciumana, F. C.; Patcasa, F.; Hahnb, T., A spillover approach to oxidation catalysis over copper and manganese mixed oxides. Chem. Eng. Proc. Process Int. 1999, 38 (4-6), 563-569.

524.Rangappa, D.; Ohara, S.; Umetsu, M.; Naka, T.; Adschiri, T., Synthesis, characterization and organic modification of copper manganese oxide nanocrystals under supercritical water. J. Supercrit. Fluids 2008, 44 (3), 441-445.

525.Liang, H.; Meng, F.; Caban-Acevedo, M.; Li, L.; Forticaux, A.; Xiu, L.; Wang, Z.; Jin, S., Hydrothermal continuous flow synthesis and exfoliation of NiCo layered double hydroxide nanosheets for enhanced oxygen evolution catalysis. Nano Lett. 2015, 15 (2), 1421-7.

526. Zhu, Y. L.; Zhou, W.; Yu, J.; Chen, Y. B.; Liu, M. L.; Shao, Z. P., Enhancing electrocatalytic activity of perovskite oxides by tuning cation deficiency for oxygen reduction and evolution reactions. Chem. Mater. 2016, 28 (6), 1691-1697.

527. Kimura, Y.; Abe, D.; Ohmori, T.; Mizutani, M.; Harada, M., Synthesis of platinum nano-particles in high-temperatures and high-pressures fluids. Coll. Surf. A-Phys. Eng. Aspects 2003, 231 (1-3), 131141.

528. Harada, M.; Abe, D.; Kimura, Y., Synthesis of colloidal dispersions of rhodium nanoparticles under high temperatures and high pressures. J. Coll. Int. Sci. 2005, 292 (1), 113-121.

529. PhD Thesis, Boldrin, P. Queen Mary University of London 2008.

530. Kim, J. R.; Myeong, W. J.; Ihm, S. K., Characteristics of $\mathrm{CeO}_{2}-\mathrm{ZrO}_{2}$ mixed oxide prepared by continuous hydrothermal synthesis in supercritical water as support of Rh catalyst for catalytic reduction of NO by CO. J. Catal. 2009, 263 (1), 123-133. 
531. Parker, J. E.; Thompson, S. P.; Cobb, T. M.; Yuan, F. J.; Potter, J.; Lennie, A. R.; Alexander, S.;

Tighe, C. J.; Darr, J. A.; Cockcroft, J. C.; Tang, C. C., High-throughput powder diffraction on beamline I11 at Diamond. J. Appl. Crystallogr. 2011, 44, 102-110.

532. Weng, X.; Tan, D.; Cao, X.; Zhang, J.; Wu, Z., Supercritical water as a feasible reaction environment for the syntheses of hybrid nanocrystallites with strong metal-support interaction. Catal. Sci.Tech. 2016, 6 (9), 2901-2904.

533. Crook, J.; Mousavi, A., The chlor-alkali process: A review of history and pollution. Envir. Forensics 2016, $17(3), 211-217$.

534. Darr, J. A.; Mills, A.; Parkin, I. P.; Cora, F.; Sankar, G., EPSRC grant ref EP/Moo8754/1 Sustainable oxidation catalysts for the production of solar hydrogen and chlorine from brine, see http://gow.epsrc.ac.uk/NGBOViewGrant.aspx?GrantRef=EP/Moo8754/1. 2014.

535. McCafferty, L.; Rourke, C. O.; Mills, A.; Kafizas, A.; Parkin, I. P.; Darr, J. A., Light-driven generation of chlorine and hydrogen from brine using highly selective Ru/Ti oxide redox catalysts. Sus. Energy Fuels 2017, Advance Article DOI: 10.1039/C6SE00057F.

536. Ormerod, R. M., Solid oxide fuel cells Chem. Soc. Rev. 2003, 32, 17-28

537. Boldrin, P.; Ruiz-Trejo, E.; Yu, J. W.; Gruar, R. I.; Tighe, C. J.; Chang, K. C.; llavsky, J.; Darr, J. A.; Brandon, N., Nanoparticle scaffolds for syngas-fed solid oxide fuel cells. J. Mater. Chem. A 2015, 3 (6), 3011-3018.

538. Weng, X.; Knowles, J. C.; Abrahams, I.; Wu, Z.; Darr, J. A., In situ variable temperature X-ray diffraction studies on the transformations of nano-precursors to La-Ni-O phases. J. Solid State Chem. 2011, 184 (7), 1688-1694.

539. PhD Thesis, Weng X. "Continuous Hydrothermal Flow Syntheses of Nanoceramics towards Better Oxide Ion and Electronic Conductors", Queen Mary University of London 2008. 
540.Zhang, Z.; Greenblatt, M.; Goodenough, J. B., Synthesis, Structure, and Properties of the Layered

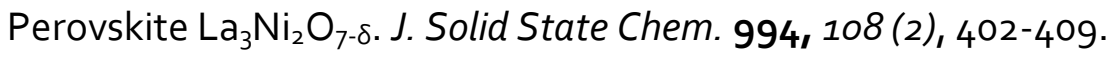

541.Zhang, Z.; Greenblatt, M., Synthesis, Structure, and Properties of $\mathrm{Ln}_{4} \mathrm{Ni}_{3} \mathrm{O}_{10-\delta}(\mathrm{Ln}=\mathrm{La}$, $\operatorname{Pr}$, and Nd). J. Solid State Chem. 1995, 117 (2), 236-246.

542.Alexander, S. J.; Lin, T.; Brett, D. J. L.; Evans, J. R. G.; Cibin, G.; Dent, A.; Sankar, G.; Darr, J. A., A combinatorial nanoprecursor route for direct solid state chemistry: Discovery and electronic properties of new iron-doped lanthanum nickelates up to $\mathrm{La}_{4} \mathrm{Ni}_{2} \mathrm{FeO}_{10-d}$. Solid State lonics 2012, $225,176-181$.

543. PhD Thesis, S. J. Alexander Discovery of Heterometallic Layered Oxides using Solid-State Reactions of Nano-Precursors, University College London 2011.

544. Ruiz-Trejo, E.; Boldrin, P.; Lubin, A.; Tariq, F.; Fearn, S.; Chater, R.; Cook, S. N.; Atkinson, A.; Gruar, R. I.; Tighe, C. J.; Darr, J. A.; Brandon, N. P., Novel composite cermet for low-metalcontent oxygen separation membranes. Chem. Mater. 2014, 26 (13), 3887-3895.

545. Okadaa, K.; Hayashi, H.; Takesue, M.; Watanabe, M.; Smith Jr., R. L., Synthesis of ferroelectric K1${ }_{x} \mathrm{Na}_{x} \mathrm{Nb}_{1-y} \mathrm{Ta}_{y} \mathrm{O}_{3}$ nanoparticles using a supercritical water flow system. J. Supercrit. Fluids 2017, $123,101-108$.

546. Grätzel, M., Dye-sensitized solar cells. J. Photochem. Photobiol. C: Photochem. Rev. 2003, 4 (2), $145^{-153}$

547. Li, X.; Qiu, Y.; Wang, S.; Lu, S.; Gruar, R. I.; Zhang, X.; Darr, J. A.; He, T., Electrophoretically deposited $\mathrm{TiO}_{2}$ compact layers using aqueous suspension for dye-sensitized solar cells Phys. Chem. Chem. Phys 2013, 15, 14729-14735

548.Burst, J. M.; Duenow, J. N.; Albin, D. S.; Colegrove, E.; Reese, M. O.; Aguiar, J. A.; Jiang, C.-S.; Patel, M. K.; Al-Jassim, M. M.; Kuciauskas, D.; Swain, S.; Ablekim, T.; Lynn K. G.; Metzger, W. K. 
CdTe solar cells with open-circuit voltage breaking the 1 V barrier, Nature Energy 2016, 1, Article number 16015 .

549. Devaraju, M. K.; Truong, Q. D.; Tomai, T.; Honm, I., Supercritical fluid methods for synthesizing cathode materials towards lithium ion battery applications. RSC Adv. 2014, 4 (52), 27452-27470.

550. Hanwha Corporation of Korea. http://hcc.hanwha.com/eng/business/bus battery.jsp.

551. see video on how to make a printed electrode https://www.youtube.com/watch?v=kVatkIDngzI\&t=635s.

552. see video on how to make a coin cell in a glovebox https://www.youtube.com/watch?v=sPT59agqsCc\&t=2s.

553. Prosini , P. P.; Gislon, P., A hydrogen refill for cellular phone. J. Power Sources 2006, 161 (1), $290-$ 293.

554. Wee, J. H., A feasibility study on direct methanol fuel cells for laptop computers based on a cost comparison with lithium-ion batteries. J. Power Sources 2007, 173 (1), 424-436.

555. Amatucci, G. G.; Pereira, N., Fluoride based electrode materials for advanced energy storage devices. J. Flu. Chem. 2007, 128 (4), 243-262.

556. Aurbach, D.; Markovsky, B.; Salitra, G.; Markevich, E.; Talyossef, Y.; Koltypin, M.; Nazar, L.; Ellis, B.; Kovacheva, D., Review on electrode-electrolyte solution interactions, related to cathode materials for Li-ion batteries. J. Power Sources 2007, 165 (2), 491-499.

557. Flipsen, S. F. J., Power sources compared: The ultimate truth? J. Power Sources 2006, 162 (2), 927934 .

558. Johnson, I. D.; Blagovidova, E.; Dingwall, P. A.; Brett, D. J. L.; Shearing, P. R.; Darr, J. A., High power $\mathrm{Nb}$-doped $\mathrm{LiFePO}_{4} \mathrm{Li}$-ion battery cathodes; pilot-scale synthesis and electrochemical properties. J. Power Sources 2016, 326, 476-481. 
559. Johnson, I. D.; Loveridge, M.; Bhagat, R.; Darr, J. A., Mapping structure-composition-property relationships in V- and Fe-Doped $\mathrm{LiMnPO}_{4}$ cathodes for lithium-ion batteries. ACS Combi. Sci. 2016, $18(11), 665-672$.

56o. Loveridge, M. J.; Lain, M. J.; Johnson, I. D.; Roberts, A.; Beattie, S. D.; Dashwood, R.; Darr, J. A.; Bhagat, R., Towards high capacity Li-ion batteries based on silicon-graphene composite anodes and sub-micron V-doped $\mathrm{LiFePO}_{4}$ cathodes. Sci. Rep. 2016, 6, Article number 37787. 561. Kanamura, K.; Dokko, K.; Kaizawa, T., Synthesis of spinel $\mathrm{LiMn}_{2} \mathrm{O}_{4}$ by a hydrothermal process in supercritical water with heat-treatment. J. Electrochem. Soc. 2005, 152 (2), A391-A395.

562. Aimable, A.; Aymes, D.; Bernard, F.; Le Cras, F., Characteristics of $\mathrm{LiFePO}_{4}$ obtained through a one step continuous hydrothermal synthesis process working in supercritical water. Solid State Ionics 2009, 180 (11-13), 861-866.

563. Jiang, C.; Hosono, E.; Zhou, H., Nanomaterials for lithium ion batteries. Nano Today 2006, 1 (4), 28-33.

564. Bartholomew, R. F.; Young, D. M.; Ellison, A. J. G., Electrical properties of new glasses based on the $\mathrm{Li}_{2} \mathrm{~S}-\mathrm{SiS}_{2}$ system. J. Non-Cryst. Solids 1999, 256-257, 242-247.

565.Raja, M. W.; Mahanty, S.; Ghosh, P.; Basu, R. N.; Maiti, H. S., Alanine-assisted low-temperature combustion synthesis of nanocrystalline $\mathrm{LiMn}_{2} \mathrm{O}_{4}$ for lithium-ion batteries. Mater. Res. Bull. 2007, $42(8), 1499-1506$.

566. Hao, Y. J.; Lai, Q. Y.; Lu, J. Z.; Liu, D. Q.; Ji, X. Y., Influence of various complex agents on electrochemical property of $\mathrm{Li}_{4} \mathrm{Ti}_{5} \mathrm{O}_{12}$ anode material. J. Alloys Compd. 2007, 439 (1-2), 330-336. 567. Liang, Y.; Fan, J.; Xia, X. h.; Luo, Y. S.; Jia, Z. j., Modified hydrothermal synthesis of $\mathrm{SnO}_{y}-\mathrm{SiO}_{2}$ for lithium-ion battery anodes. Electrochim. Acta 2007, 52 (19), 5891-5895. 
568. Kanamura, K.; Goto, A.; Ho, R. Y.; Umegaki, T.; Toyoshima, K.; Okada, K.; Hakuta, Y.; Adschiri, T.; Arai, K., Preparation and electrochemical characterization of $\mathrm{LiCoO}_{2}$ particles prepared by supercritical water synthesis. Electrochem. Solid State Letts 2000, 3 (6), 256-258.

569. Shin, Y. H.; Koo, S. M.; Kim, D. S.; Lee, Y. H.; Veriansyah, B.; Kim, J.; Lee, Y. W., Continuous hydrothermal synthesis of $\mathrm{HT}-\mathrm{LiCoO}_{2}$ in supercritical water. J. Supercrit. Fluids 2009, 50 (3), 250256.

570. Lee, J. H.; Ham, J. Y., Synthesis of manganese oxide particles in supercritical water. Korean J. Chem. Eng. 2006, 23 (5), 714-719.

571. Padhi, A. K.; Nanjundaswamy, K. S.; Goodenough, J. B., Phospho-olivines as positive-electrode materials for rechargeable lithium batteries. J. Electrochem. Soc 1997, 144 (4), 1188-1194.

572. Jiang, J.; Dahn, J. R., ARC studies of the thermal stability of three different cathode materials: $\mathrm{LiCoO}_{2 i} \mathrm{Li}\left[\mathrm{Ni}_{0.1} \mathrm{CO}_{0.8} \mathrm{Mn}_{0.1}\right] \mathrm{O}_{2 i}$ and $\mathrm{LiFePO}_{41}$ in $\mathrm{LiPF}_{6}$ and LiBoB EC/DEC electrolytes. Electrochem. Commun. 2004, 6 (1), 39.

573. Maa, J.; Lia, B.; Kanga, F., Improved electrochemical performances of nanocrystalline $\mathrm{LiFePO}_{4} / \mathrm{C}$ composite cathode via V-doping and $\mathrm{VO}_{2}(\mathrm{~B})$ coating. J. Phys. Chem. Solids 2012, 73 (12), 14631468.

574. Hong, S. A.; Kim, S. J.; Kim, J.; Lee, B. G.; Chung, K. Y.; Lee, Y. W., Carbon coating on lithium iron phosphate $\left(\mathrm{LiFePO}_{4}\right)$ : Comparison between continuous supercritical hydrothermal method and solid-state method. Chem. Eng. J. 2012, 198, 318-326.

575. Hong, S. A.; Kim, S. J.; Kim, J.; Chung, K. Y.; Cho, B. W.; Kang, J. W., Small capacity decay of lithium iron phosphate $\left(\mathrm{LiFePO}_{4}\right)$ synthesized continuously in supercritical water: comparison with solid-state method. J. Supercrit. Fluids 2011, 55 (3), 1027-1037.

576. Yu, W. L.; Zhao, Y. P.; Rao, Q. L., Rapid and Continuous production of LiFePO 4 /C nanoparticles in superheated water. Chin. J. Chem. Eng. 2009, 17 (1), 171-174. 
577. Xu, C.; Lee, J.; Teja, A. S., Continuous hydrothermal synthesis of lithium iron phosphate particles in subcritical and supercritical water. J. Supercrit. Fluids 2008, 44 (1), 92-97.

578. Park, O. K.; Cho, Y.; Lee, S.; Yoo, H.-C.; Song, H.-K.; Cho, J., Who will drive electric vehicles, olivine or spinel? Energy Environ. Sci. 2011, 4 (5), 1621-1633

579. Park, M.; Zhang, X.; Chung, M.; Less, G. B.; Sastry, A. M., A review of conduction phenomena in Li-ion batteries. J. Power Sources 2010, 195 (24), 7904-7929.

580. Howard, W. F.; Spotnitz, R. M., Theoretical evaluation of high-energy lithium metal phosphate cathode materials in Li-ion batteries. J. Power Sources 2007, 165 (2), 887-891.

581. Lee, J.; Teja, A. S., Characteristics of lithium iron phosphate $\left(\mathrm{LiFePO}_{4}\right)$ particles synthesized in subcritical and supercritical water. J. Supercrit. Fluids 2005, 35 (1), 83-90.

582.Lee, J.; Teja, A. S., Synthesis of $\mathrm{LiFePO}_{4}$ micro and nanoparticles in supercritical water. Mater. Lett. 2006, 60 (17-18), 2105-2109.

583. Lübke, M.; Shin, J.; Marchand, P.; Brett, D.; Shearing, P.; Liu, Z.; Darr, J. A., Highly pseudocapacitive $\mathrm{Nb}$-doped $\mathrm{TiO}_{2}$ high power anodes for lithium-ion batteries. J. Mater. Chem. A 2015, 3 (45), 22908-22914.

584. Lübke, M.; Ding, N.; Powell, M. J.; Brett, D. J.; Shearing, P. R.; Liu, Z.; Darr, J. A., VO 2 nanosheet negative electrodes for lithium-ion batteries. Electrochem. Commun. 2016, 64, 56-6o. 585. Hong, S. A.; Nugroho, A.; Kim, S. J.; Kim, J.; Chung, K. Y.; Cho, B. W.; Kang, J. W., Continuous supercritical hydrothermal synthesis: lithium secondary ion battery applications. Res. Chem. Intermed. 2011, $37(2-5), 429-440$.

586. Johnson, I. D.; Lubke, M.; Wu, O. Y.; Makwana, N. M.; Smales, G. J.; Islam, H. U.; Dedigama, R. Y.; Gruar, R. I.; Tighe, C. J.; Scanlon, D. O.; Corà, F.; Brett, D. J. L.; Shearing, P. R.; Darr, J. A., Pilot-scale continuous synthesis of a vanadium-doped $\mathrm{LiFePO}_{4} / \mathrm{C}$ nanocomposite high-rate cathodes for lithium-ion batteries J. Power Sources 2015, 302, 410-418. 
587. Wang, Z. H.; Yuan, L. X.; Zhang, W. X.; Huang, Y. H., LiFe $e_{0.8} \mathrm{Mn}_{0.2} \mathrm{PO}_{4} / \mathrm{C}$ cathode material with high energy density for lithium-ion batteries. J. Alloys Compd. 2012, 532, 25-30.

588.Su, L.; Jing, Y.; Zhou, Z., Li ion battery materials with core-shell nanostructures. Nanoscale 2011, 3 (10), 3967-3983.

589. Amalraj, F.; Kovacheva, D.; Talianker, M.; Zeiri, L.; Grinblat, J.; Leifer, N.; Goobes, G.;

Markovsky, B.; Aurbach, D., Synthesis of integrated cathode materials

$x \mathrm{Li}_{2} \mathrm{MnO}_{3}(1-\mathrm{x}) \mathrm{LiMn}_{1 / 3} \mathrm{Ni}_{1 / 3} \mathrm{CO}_{1 / 3} \mathrm{O}_{2}(\mathrm{x}=0.3,0.5,0.7)$ and studies of their electrochemical behavior.

J. Electrochem. Soc. 2010, 157 (10), S19-S19.

590. Reddy, M. V.; Rao, G. V. S.; Chowdari, B. V. R., Metal oxides and oxysalts as anode materials for Li-ion batteries. Chem. Rev. 2013, 113 (7), 5364-5457.

591.Pang, Q.; Liang, X.; Kwok, C. Y.; Nazar, L. F., Advances in lithium-sulfur batteries based on multifunctional cathodes and electrolytes. Nature Energy 2016, 1, Article number 16132.

592. Yermukhambetova, A.; Bakenov, Z.; Zhang, Y.; Darr, J. A.; Brett, D. J. L.; Shearing, P. R., Examining the effect of nanosized $\mathrm{Mg}_{0.6} \mathrm{Ni}_{0.4} \mathrm{O}$ and $\mathrm{Al}_{2} \mathrm{O}_{3}$ additives on S/polyaniline cathodes for lithium-sulphur batteries. J. Electroanal. Chem. 2016, 780 (1), 407-415.

593.Park, M.; Kim, K. Y.; Seo, H.; Cheon, Y. E.; Koh, J. H.; Sun, H.; Kim, T. J., Practical challenges associated with catalyst development for the commercialization of Li-air batteries. J. Electrochem. Sci. Tech.014, 5 (1), 1-18.

594. Liu, M.; Rong, Z. Q.; Malik, R.; Canepa, P.; Jain, A.; Ceder, G.; Persson, K. A., Spinel compounds as multivalent battery cathodes: a systematic evaluation based on ab initio calculations. Energy Environ. Sci. 2015, 8 (3), 964-974.

595.Shao, Y.; Liu, T.; Li, G.; Gu, M.; Nie, Z.; Engelhard, M.; Xiao, J.; Lv, D.; Wang, C.; Zhang, J.-G.; Liu, J., Coordination chemistry in magnesium battery electrolytes: how ligands affect their performance. Sci. Rep. 2013, 3, Article number 3130. 
596. Levi, E.; Gofer, Y.; Aurbach, D., On the way to rechargeable Mg batteries: the challenge of new cathode materials. Chem. Mat 2010, 22, 860-868.

597. Etacheri, V.; Marom, R.; Elazari, R.; Salitra, G.; Aurbach, D., Challenges in the development of advanced Li-ion batteries: a review. Energy Environ. Sci. 2011, 4 (9), 3243-3262.

598. Poizot, P.; Laruelle, S.; Grugeon, S.; Dupont, L.; Tarascon, J. M., Nano-sized transitionmetaloxides as negative-electrode materials for lithium-ion batteries. Nature 2000, 407 (6803), 496-499.

599. Zhang, W. J., A review of the electrochemical performance of alloy anodes for lithium-ion batteries. J. Power Sources 2011, 196 (1), 13-24.

6oo. Goriparti, S.; Miele, E.; De Angelis, F.; Di Fabrizio, E.; Zaccaria, R. P.; Capiglia, C., Review on recent progress of nanostructured anode materials for Li-ion batteries. J. Power Sources 2014, $257,421-443$.

601.Laumann, A.; Bremholm, M.; Hald, P.; Holzapfel, M.; Fehr, K. T.; Iversen, B. B., Rapid Green Continuous Flow Supercritical Synthesis of High Performance $\mathrm{Li}_{4} \mathrm{Ti}_{5} \mathrm{O}_{12}$ Nanocrystals for Li Ion Battery Applications. J. Electrochem. Soc. 2012, 159 (2), A166-A171.

602. Hayashi, $\mathrm{H}_{\text {; }}$ Nakamura, T.; Ebina, T., Hydrothermal synthesis of $\mathrm{Li}_{4} \mathrm{Ti}_{5} \mathrm{O}_{12}$ nanoparticles using a supercritical flow reaction system. J. Ceram. Soc. Jpn. 2014, 122 (1421), 78-82.

603.Lubke, M.; Sumboja, A.; Johnson, I. D.; Brett, D. J. L.; Shearing, P. R.; Liu, Z. L.; Darr, J. A., High power nano- $\mathrm{Nb}_{2} \mathrm{O}_{5}$ negative electrodes for lithium-ion batteries. Electrochim. Acta 2016, 192, $363-369$.

604. Luebke, M.; Ding, N.; Powell, M. J.; Brett, D. J. L.; Shearing, P. R.; Liu, Z. L.; Darr, J. A., VO2 nano-sheet negative electrodes for lithium-ion batteries. Electrochem. Commun. 2016, 64, 56-6o. 
605. Lübke, M.; Howard, D.; Armer, C.; Gardecka, A. J.; Lowe, A.; Reddy, M. V. V.; Liu, Z.; Darr, J. A., Combination of alloy and insertion nanomaterials for high energy lithium ion batteries. J. Power Sources 2016, under review.

6o6. Fang, Z.; Assaaoudi, H.; Lin, H.; Wang, X.; Butler, I. S.; Kozinski, J. A., Synthesis of nanocrystalline $\mathrm{SnO}_{2}$ in supercritical water. J. Nanopart. Res. 2007, 9 (4), 683-687.

607. Nørby, P.; Roelsgaard, M.; Søndergaard, M.; Iversen, B. B., Hydrothermal synthesis of $\operatorname{CoSb}_{2} \mathrm{O}_{4}$ : in situ powder X-ray diffraction, crystal structure, and electrochemical properties. Cryst. Growth Des. 2016, $16(2), 834-841$.

6o8. Naoi, K.; Simon, P., New Materials and New Configurations for Advanced Electrochemical Capacitors. Electrochem. Soc. Int. 2008, 17 (1), 34-37.

6o9. Augustyn, V.; Simon, P.; Dunn, B., Pseudocapacitive oxide materials for high-rate electrochemical energy storage. Energy Environ. Sci. 2014, 7 (5), 1597.

610.Sue, K.; Kawasaki, S. i.; Suzuki, M.; Hakuta, Y.; Hayashi, H.; Arai, K.; Takebayashi, Y.; Yoda, S.; Furuya, T., Continuous hydrothermal synthesis of $\mathrm{Fe}_{2} \mathrm{O}_{3}, \mathrm{NiO}$, and $\mathrm{CuO}$ nanoparticles by superrapid heating using a T-type micro mixer at 673 K and $30 \mathrm{MPa}$. Chem. Eng. J. 2011, 166 (3), $947-953$

611. Nugroho, A.; Kim, J., Effect of $\mathrm{KOH}$ on the continuous synthesis of cobalt oxide and manganese oxide nanoparticles in supercritical water. J. Ind. Eng. Chem. 2014, 20 (6), 4443-4446.

612.King'ondu, C. K.; Opembe, N. N.; Genuino, H. C.; Garces, H. F.; Njagi, E. C.; lyer, A.; Huang, H.; Dharmarathna, S.; Suib, S. L., Nonthermal synthesis of three-dimensional metal oxide structures under continuous-flow conditions and their catalytic applications. J. Phys. Chem. C 2011, 115 (47), $23273-23282$.

613. Moro, F.; Tang, S. V. Y.; Tuna, F.; Lester, E., Magnetic properties of cobalt oxide nanoparticles synthesised by a continuous hydrothermal method. J. Magn. Magn. Mater. 2013, 348, 1-7. 
614.Jeon, H. S.; Nugroho, A.; Kim, J.; Kim, H.; Min, B. K., Size-dependent electrocatalytic activities of printed $\mathrm{CO}_{3} \mathrm{O}_{4}$ films for a monolithic photovoltaic-electrolytic hydrogen generation system. Int. J. Hydrogen Energy 2011, 36 (17), 10587-10592.

615. Proesmans, P. I.; Luan, L.; Buelow, S. J., Hydrothermal oxidation of organic wastes using ammonium nitrate. Ind. Eng. Chem. Res. 1997, 36 (5), 1559-1566.

616.Phillips, M. J.; Duncanson, P.; Wilson, K.; Darr, J. A.; Griffiths, D. V.; Rehman, I. U., Surface modification of bioceramics by grafting of tailored ally phosphonic acid Adv. Appl. Ceram. 2005, $104(5), 261-267$.

617. Bayliss, P. A.; Ibarra, I. A.; Perez, E.; Yang, S.; Tang, C. C.; Poliakoff, M.; Schröder, M., Synthesis of metal-organic frameworks by continuous flow. Green Chem. 2014, 16, 3796-3802.

618. Rubio-Martinez, M.; Batten, M. P.; Polyzos, A.; Carey, K.-C.; Mardel, J. I.; Lim, K. S.; Hill, M. R., Versatile, high quality and scalable continuous flow production of metal-organic frameworks. Sci. Rep. 2014, 4, Article number 5443.

619.D'Arras L; Sassoye C; Rozes L; Sanchez C; Marrot J; Marre S; C, A., Fast and continuous processing of a new sub-micronic lanthanide-based metal-organic framework. New J. Chem 2014 $38(4), 1477-1483$.

620. Dunne, P. W.; Lester, E.; Walton, R. I., Towards scalable and controlled synthesis of metalorganic framework materials using continuous flow reactors.

React. Chem. Eng. 2016, 1 (4), 352-360.

621.Padmajan, S.; Poulin, P.; Aymonier, C., Prospects of supercritical fluids in realizing graphene based functional materials. Adv. Mater. 2016, 28, 2663-2691.

622. Pascu, O.; Marre, S.; Aymonier, C., Creation of interfaces in composite/hybrid nanostructured materials using Supercrit. Fluids. Nanotech. Rev. 2015, 4 (6), 487-515. 
623. Elbasuney, S.; Mostafa, H. E., Synthesis and surface modification of nanophosphorous-based flame retardant agent by continuous flow hydrothermal synthesis. Particuology 2015, 22, 82-88.

624. Takami, S.; Ohara, S.; Adschiri, T.; Wakayama, Y.; Chikyow, T., Continuous synthesis of organic-inorganic hybridized cubic nanoassemblies of octahedral cerium oxide nanocrystals and hexanedioic acid. Dalton Trans. 2008, (40), 5442-5446.

625. Litwinowicz, A. A.; Takami, S.; Hojo, D.; Aoki, N.; Adschiri, T., Hydrothermal Synthesis of cerium oxide nanoassemblies through coordination programming with amino acids. Chem. Lett. 2014, 43 (8), 1343-1345.

626. Giroire, B.; Slostowski, C.; Marre, S.; Aymonier, C.; Aida, T.; Hojo, D.; Aoki, N.; Takami, S.; Adschiri, T., Tuning surface grafting density of $\mathrm{CeO}_{2}$ nanocrystals with near- and supercritical solvent characteristics. PCCP 2016, 18 (3), 1727-34.

627.http://www.itec-es.co.jp/English/product/momi_mini.html.

628. Adschiri, T.; Takami, S.; Minami, K.; Yamagata, T.; Miyata, K.; Morishita, T.; Ueda, M.; Fukushima, K.; Ueno, M.; Okada, T.; Oshima, H.; Mitani, Y.; Asahina, S.; Unno S. Online article http://citeseerx.ist.psu.edu/viewdoc/download?doi=10.1.1.464.6279\&rep=rep1\&type=pdf

629. http://www.itec-es.co.jp/English/index.html.

630. Elbasuney, S.; Mostafa, S. F., Continuous flow formulation and functionalization of magnesium di-hydroxide nanorods as a clean nano-fire extinguisher. Powder Technol. 2015, 278, 72-83.

631. Elbasuney, S. PhD thesis, Enhanced flame retardant polymer nanocomposites, University of Nottingham. 2013. http://eprints.nottingham.ac.uk/14587/1/594394.pdf

632. Hellstern, H. L.; Mamakhel, A.; Bremholm, M.; Iversen, B. B., Core-shell nanoparticles by silica coating of metal oxides in a dual-stage hydrothermal flow reactor. Chem Commun 2016, 52 (16), 3434-7. 
633. Elbasuney, S., Continuous hydrothermal synthesis of $\mathrm{AlO}(\mathrm{OH})$ nanorods as a clean flame retardant agent. Particuology 2015, 22, 66-71.

634.Dumas, A.; Claverie, M.; Slostowski, C.; Aubert, G.; Careme, C.; Roux, C. L.; Micoud, P.; Martin, F.; Aymonier, C., Fast-Geomimicking using Chemistry in SupercriticalWater. Angew. Chem. 2016, $2016(128), 1-5$

635. Ciullo, P. A. Industrial Minerals and Their Uses: A Handbook and Formulary. Publ. William Andrew 1996.

636.Zhang, F.; Robinson, B. W.; de Villiers-Lovelock, H.; Wood, R. J. K.; Wang, S. C., Wettability of hierarchically-textured ceramic coatings produced by suspension HVOF spraying J. Mater. Chem. A 2015, 3, 13864-13873.

637. Tyrsted, C.; Becker, J.; Hald, P.; Bremholm, M.; Pedersen, J. S.; Chevallier, J.; Cerenius, Y.; Iversen, S. B.; Iversen, B. B., In-Situ synchrotron radiation study of formation and growth of crystalline $\mathrm{Ce}_{x} \mathrm{Zr}_{1-\mathrm{x}} \mathrm{O}_{2}$ nanoparticles synthesized in supercritical water. Chem. Mater. 2010, 22 (5), 1814-1820.

638.Bremholm, M.; Jensen, H.; Iversen, S. B.; Iversen, B. B., Reactor design for in situ X-ray scattering studies of nanoparticle formation in supercritical water syntheses. J. Supercrit. Fluids 2008, 44 (3), 385-390.

639. Yoko, A.; Akizuki, M.; Hirao, N.; Kohara, S.; Kumar, M.; Umezawa, N.; Ohno, T.; Oshima, Y., In situ X-ray diffraction for millisecond-order dynamics of $\mathrm{BaZrO}_{3}$ nanoparticle formation in supercritical water. J. Supercrit. Fluids 2016, 107, 746-752.

640. Middelkoop, V.; Tighe, C. J.; Kellici, S.; Gruar, R. I.; Perkins, J. M.; Jacques, S. D. M.; Barnes, P.; Darr, J. A., Imaging the continuous hydrothermal flow synthesis of nanoparticulate $\mathrm{CeO}_{2}$ at different supercritical water temperatures using in situ angle-dispersive diffraction. J. Supercrit. Fluids 2014, 87, 118-128. 
641.Middelkoop, V.; Boldrin, P.; Peel, M.; Buslaps, T.; Barnes, P.; Darr, J. A.; Jacques, S. D. M., Imaging the inside of a continuous nanoceramic synthesizer under supercritical water conditions using high-energy synchrotron X-radiation. Chem. Mater. 2009, 21 (12), 2430-2435.

642. Textbook of Drug Design and Discovery. . Taylor \& Francis Washington, DC, 2002.

643.Gao, C.; Bao, J.; Luo, Z. L.; Liu, W. H., Recent progresses in the combinatorial materials science. Acta Phys.Chim. Sin. 2006, 22 (7), 899-912.

644. Hattrick-Simpers, J.; Wen, C.; Lauterbach, J., The materials super highway: integrating highthroughput experimentation into mapping the catalysis materials genome. Catal. Lett. 2015, 145 (1), $290-298$.

645. Koinuma, H.; Takeuchi, I., Combinatorial solid-state chemistry of inorganic materials. Nature Mater. 2004, $3(7), 429-438$.

646. Spaller, M. R.; Burger, M. T.; Fardis, M.; Bartlett, P. A., Synthetic strategies in combinatorial chemistry. Curr. Opin. Chem. Biol. 1997, 1 (1), 47-53.

647.Wang, J.; Evans, J. R., London University Search Instrument: a combinatorial robot for highthroughput methods in ceramic science. J. Comb. Chem. 2005 7(5), 665-672.

648. Kellici, S.; Gong, K.; Lin, T.; Brown, S.; Clark, R. J.; Vickers, M.; Cockcroft, J. K.; Middelkoop, V.; Barnes, P.; Perkins, J. M.; Tighe, C. J.; Darr, J. A., High-throughput continuous hydrothermal flow synthesis of Zn-Ce oxides: unprecedented solubility of $\mathrm{Zn}$ in the nanoparticle fluorite lattice. Philosophical Trans. Roy. Soc. A-Math. Phys. Eng Sci. 2010, 368 (1927), 4331-4349.

649. Technology Readiness Levels see web https://www.nasa.gov/directorates/heo/scan/engineering/technology/txt_accordionı.html.

650. Recovery of precious metal catalysts with supercritical water oxidation. Filt. Sep. 2003, 40 (5), 16-18. 
651. Veriansyah, B.; Kim, J. D., Supercritical water oxidation for the destruction of toxic organic wastewaters: A review J. Environ. Sci. 2010, 19 (5), 513-522.

652. Savage, P. E., Organic chemical reactions in supercritical water. Chem. Rev. 1999, 99 (2), 603621.

653. AQUCAT process see weblink http://www.platinummetalsreview.com/pdf/163-166-pmrocto3.pdf 2010.

654. Behrendt, F.; Neubauer, Y.; Oevermann, M.; Wilmes, B.; Zobel, N., Direct liquefaction of biomass. Chem. Eng. Technol. 2008, 31 (5), 667-677.

655.Loppinet-Serani, A.; Aymonier, C.; Cansell, F., Supercritical water for environmental technologies. J. Chem. Technol. Biotechnol. 2010, 85 (5), 583-589.

656. Toor, S. S.; Rosendahl, L.; Rudolf, A., Hydrothermal liquefaction of biomass: A review of subcritical water technologies. Energy 2011, 36 (5), 2328-2342.

657. A., L.-S.; Aymonier, C., Hydrolysis in Near- and Supercritical Water for Biomass Conversion and Material Recycling. Elsevier: 2014; 139-156.

658. Knez, Z.; Markocic, E.; Leitgeb, M.; Primozic, M.; Knez Hrncic, M.; Skerget, M., Industrial applications of supercrit. fluids: a review. Energy 2014, 77, 235-243.

659. Naik, A. J.; Gruar, R.; Tighe, C. J.; Parkin, I. P.; Darr, J. A.; Binions, R., Environmental sensing semiconducting nanoceramics made using a continuous hydrothermal synthesis pilot plant. Sens. Act. B: Chem. 2015, 217, 136-145.

66o. Tsang, M.; Philippot, G.; Aymonier, C.; Sonnemann, G., Anticipatory life-cycle assessment of supercritical fluid synthesis of barium strontium titanate nanoparticles. Green Chem. 2016, 18, 4924-4933 
661.Marre, S.; Adamo, A.; Basak, S.; Aymonier, C.; Jensen, K. F., Design and packaging of microreactors for high pressure and high temperature applications. Ind. Eng. Chem. Res. 2010, 49 (22), 11310-11320.

662. Takami, S.; Sugioka, K.-i.; Ozawa, K.; Tsukada, T.; Adschiri, T.; Sugimoto, K.; Takenaka, N.; Saito, Y., In-situ neutron tomography on mixing behavior of supercritical water and room temperature water in a tubular flow reactor. Physics Proc. 2015, 69, 564-569.

663. Li, H.; Li, C.; Jiao, C.; Wang, S. Characterization of cerium dioxide nanoparticles prepared by supercritical water oxidation, Ceram. Int. 2015, 41 (8), 10170-10176.

664 Zhu, Y.; Takami, S.; Seong, G.;

Dejhosseini, M.; Hossain, M. Z.; Noguchi ,T.; Hojo, D.; Aoki, N.; Aida, T.; Adschiri, T. Green solvent for green materials: a supercritical hydrothermal method and shape-controlled synthesis of $\mathrm{Cr}$-doped $\mathrm{CeO}_{2}$ nanoparticles. Phil.Trans. R. Soc. A 2015, 373: Article number 20150012.

665 Weng, X.; Zhang, J.; Wu, Z.; Liu, Y.; Wang, H.; Darr, J. A.Continuous syntheses of highly dispersed composite nanocatalysts via simultaneous co-precipitation in supercritical water Appl. Cat. B:

Environ. 2011, 103, 453-461.

666 Kriedemann, B.; Fester, V. Critical process parameters and their interactions on the continuous hydrothermal synthesis of ironoxide nanoparticles. Chem. Eng. J. 2015, 281, 312-321.

667 Lee, H. C.; Kim, J. H. ; In, J. H.; Lee, C. H. NaFeEDTA decomposition and hematite nanoparticle formation in supercritical water oxidation, Ind. Eng. Chem. Res. 2005, 44, 6615-6621.

668 Zielke, P.; Xu, Y.; Simonsen, S. B.; Norby, P.; Kiebach, R.Simulation, design and proof-of-concept of a two-stage continuous hydrothermal flow synthesis reactor for synthesis of functionalizednano-sized inorganic composite materials. J. of Supercrit. Fluids 2016, 117, 1-12 669. Chua, G.; Zeng, Q.; Shena, Z.; Zoua, H.; J Chena, J. Chem. Eng. J. 2014, 253, 78-83 
670. Ohara, S.; Mousavand, T.; Sasaki, T.; Umetsu, M.; Naka, T.; Adschiri, T. Continuous production of fine zinc oxide nanorods by hydrothermal synthesis in supercritical water. J. Mater Sci. 2008, 43, $2393-2396$.

671. Levy, C.; Watanabe, M.; Aizawa, Y.; Inomata, H. Synthesis of Nanophased Metal Oxides in Supercritical Water: Catalysts for Biomass Conversion Int. J. Appl. Ceram. Technol. 2006, 3, 337344.

672. Robinson, B. ; Tabecki, A.; Paul, S.; Shi, G.; Mills, A.; Parkin, I. P.; Darr, J. A.; Lovelock, H.L.D. A Comparison of the Potential Capability of SFS, SPS and HVSFS for the Production of Photocatalytic Titania Coatings, J. Thermal Spray Tech. 2017, 26 , 161-172.

673. 
Professor Jawwad A. Darr is Professor of Materials Chemistry Department of Chemistry, Vice Dean of Enterprise (Maths and Physical Sciences Faculty) and head of the Clean Materials Technology group at University College London (UCL) [www.ucl.me.uk]. He received his doctorate from Imperial College in 1996 in air sensitive synthetic metal-organic chemistry. He then worked as postdoctoral researcher at Imperial College (1 year) developing CVD precursors and at the University of Nottingham (3.5 years) developing catalysts and co-developing a prototype hydrothermal flow reactor with for NP syntheses. He then worked at the Interdisciplinary Research Center (IRC) in Biomedical Materials at Queen Mary, London. From 2001 - 2006, he was holder of an EPSRC Advanced Research fellowship. He was appointed to University College London in 2007 as a Reader and promoted to full professor in October 2011. His research group develops applications of engineered NPs from continuous processes including hydrothermal flow reactors (energy storage materials, fuel cells, transparent conductive oxides, catalysts, electromagnetic applications, etc.). His team has also developed high throughput approaches to nanoceramics synthesis as well as a pilot scale continuous flow reactor (up to $2 \mathrm{~kg} \cdot \mathrm{h}^{-1}$ scale). Professor Darr has strong interactions with industry, is a member of the Nanomaterials Environment and Health Industry Group (NEHIG) and a visiting professor at Zhejiang University in China, as well as The IRC in Biomedical Materials at COMSATS University (Lahore, Pakistan).

Dr Neel Makwana is an industrial research scientist at Johnson Matthey currently working in catalysis. Prior to beginning this position in October 2015, he completed a PhD under the supervision of Professor Jawwad Darr, in the Clean Materials Technology Group at the Department of Chemistry, University College London (UCL). His PhD research focused on the continuous hydrothermal synthesis of nanomaterials for photocatalytic applications. Prior to his PhD, he was awarded an MSci Chemistry degree in 2011, also from UCL.

Dr. JingYi Zhang received his doctorate from Zhejiang University in 2015 in Environmental Engineering (he was also a visiting student in Professor Darr's group, for 6 months). His research interests are the syntheses of environmental nano-catalysts for volatile organic compounds (VOCs) control via continuous hydrothermal synthesis routes. 
Dr Xiaole Weng is Associate Professor of Environmental Engineering at Zhejiang University, P. R. China. He received his doctorate from University of London in 2008 in Green synthetic chemistry. In 2009, he worked as postdoctoral researcher at Zhejiang University, developing inorganic nano-catalysts for air pollution control and then became a faculty member in 2011. Dr. Weng was promoted as associate Professor in 2012. His research focuses on exploring novel properties of nano-precipitates from supercritical water, aiming to develop highly active environmental nano-catalysts. 